\title{
Radionuclide Air Emissions Report for the Hanford Site Calendar Year 1997
}

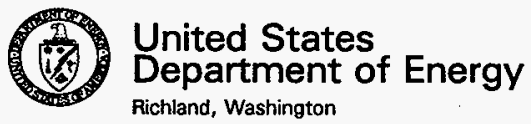


TRADEMAR DECLAMER.

Roference herein to any specific commercial product, process, or service by trade name, tredemark, manufacturer, or othorwise, does not necessarily constitute or imply its endorsement, recommendation, or favoring by the United States Govermment or any agency thereof or its contractors or subcontractore.

This report has been reproduced from the best available copy. Avalable in paper copy and microfiche.

Available to the U.S. Department of Energy

and its contractors from

U.S. Department of Energy

Office of Scientific and Tochnical Information (OST)

P.O. Box 62

Oak Ridge, TN 37831

(615) 576-8401

Available to the public from the U.S. Depertment of Commerce National Technical Information Service (NTIS)

5285 Port Royal Road

Springfield, VA 22161

(703) $487-4650$

Printed in the United States of Americe

DISCLM-5.CHP (8-95) 


\section{Radionuclide Air Emissions Report for the Hanford Site Calendar Year 1997}

B. P. Gleckler

Waste Management Federal Services of Hanford, Inc.

K. Rhoads

Pacific Northwest National Laboratory

Date Published

June 1998

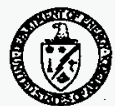

United States

Department of Energy

P.0. Box 550

Richland, Washington 99352

Approved for Public Release 


\section{RELEASE AUTHORIZATION}

Document

\section{Number:}

Document

Title:
DOE/RL-98-33, Rev. 0

\section{This document, reviewed in accordance with DOE Order 1430.1D, "Scientific and Technical Information Management," and DOE G 1430.1D-1, "Guide to the Management of Scientific and Technical Information," does not contain classified or sensitive unclassified information and is:}

\section{APPROVED FOR PUBLIC RELEASE}

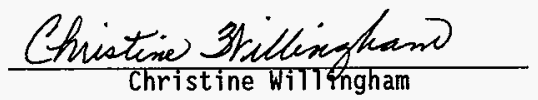

Lockheed Martin Services, Inc.

Document Control/Information Clearance

Reviewed for Applied Technology, Business Sensitive, Classified, Copyrighted, Export Controlted, Patent, Personal/Private, Proprietary, Protected CRADA, Tradenark, Unclassified Controlled Nuclear Information.

LEGAL DISCLAIMER. This report was prepared as an account of work sponsored by an agency of the United States Government. Neither the United States Government nor any agency thereof, not any of their employees, nor any of their contractors, subcontractors or their employees, makes any warranty, express or impljed, or assumes any legal liability or responsibility for the accuracy, completeness, or any third perty's use or the results of such use of any infornation, apparatus, product, or process disclosed, or represents that its use would not infringe privately owned rights. Reference herein to any specific comercial product, process, or service by trade name, trademark, manufacturer, or otherwise, does not necessarily constitute or imply its endorsenent, recomendation, or favoring. by-the. United States.Government. or. any agency. thereof or its contractors or subcontractors. The vieus and opinions of authors expressed herein do not necessarily state or reflect those of the United States Government or any agency thereof. This report has been reproduced from the best available copy. Printed in the United States of America. 


\title{
RADIONUCLIDE AIR EMISSIONS REPORT FOR THE HANFORD SITE, CALENDAR YEAR 1997
}

\begin{abstract}
This report documents radionuclide air emissions from the Hanford Site in 1997, and the resulting effective dose equivalent to the maximally exposed member of the public, referred to as the "MEI." The report has been prepared in accordance with reporting requirements in the Code of Federal Regulations, Title 40, Protection of the Environment, Part 61, "National Emissions Standards for Hazardous Air Pollutants, Subpart H, "National Emission Standards for Emissions of Radionuclides Other than Radon from Department of Energy Facilities." This report has also been prepared in accordance with the reporting requirements of the Washington Administrative Code Chapter 246-247, "Radiation Protection-Air Emissions. "
\end{abstract}

The effective dose equivalent to the MEI from the Hanford Site's 1997 point source emissions was $1.2 \mathrm{E}-03$ mrem $(1.2 \mathrm{E}-05 \mathrm{mSv})$, which is well below the 40 CFR 61 Subpart $H$ regulatory limit of $10 \mathrm{mrem} / \mathrm{yr}$. Radon and thoron emissions, exempted from $40 \mathrm{CFR} 61$ Subpart $H$, resulted in an effective dose equivalent to the $M E I$ of 2.5 E-03 mrem $(2.5$ E-05 $m S v)$. The effective dose equivalent to the MEI attributable to diffuse and fugitive emissions was $2.2 \mathrm{E}-02 \mathrm{mrem}(2.2 \mathrm{E}-04 \mathrm{mSv})$. The total effective dose equivalent from all of the Hanford Site's air emissions was $2.6 \mathrm{E}-02 \mathrm{mrem}(2.6 \mathrm{E}-04 \mathrm{mSv})$. The effective dose equivalent from all of the Hanford Site's air emissions is well below the Washington Administrative Code, Chapter 246-247, regulatory limit of $10 \mathrm{mrem} / \mathrm{yr}$.

Mr. Hector M. Rodriguez, of the U.S. Department of Energy, Richland Operations Office, can be contacted by phone, at (509) 376-6421, concerning the information in this document. 
DOE/RL-98-33

This page intentionally left blank. 


\section{CONTENTS}

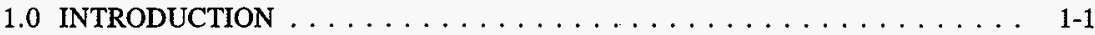

1.1 PURPOSE AND SCOPE $\ldots \ldots \ldots \ldots \ldots \ldots \ldots \ldots \ldots \ldots \ldots \ldots$

1.2 SITE DESCRIPTION $\ldots \ldots \ldots \ldots \ldots \ldots \ldots \ldots \ldots$. $\ldots \ldots \ldots$

1.2.1 Historical Background $\ldots \ldots \ldots \ldots \ldots \ldots \ldots \ldots \ldots \ldots$ 1-1

1.2.2 Major Activities . . . . . . . . . . . . . . . 1-1

1.3 POINT SOURCE DESCRIPTIONS $\ldots \ldots \ldots \ldots \ldots \ldots \ldots \ldots \ldots \ldots$

1.3.1 General Description and Reporting Criteria $\ldots \ldots \ldots \ldots \ldots \ldots$. . . . .

1.3.2 100 Areas Facilities . . . . . . . . . . . . . . . 1-6

1.3.3 200 East Area Facilities . . . . . . . . . . . . . . . . . . . . . 1-9

1.3.4 200 West Area Facilities . . . . . . . . . . . . . . . . . 1-14

1.3.5 300 Area Facilities . . . . . . . . . . . . . . . . . . 1-18

1.3.6 400 Area Facilities . . . . . . . . . . . . . . . . . . . 1-24

2.0 RADIONUCLIDE AIR EMISSIONS DATA ON POINT SOURCES $\ldots \ldots \ldots$. $\ldots$

3.0 POINT SOURCE EMISSION DOSE ASSESSMENTS . . . . . . . . . . . . 3-1

3.1 DESCRIPTION OF POINT SOURCE EMISSIONS DOSE MODEL . . . . 3 3-1

3.2 SUMMARY OF INPUT PARAMETERS $\ldots \ldots \ldots \ldots \ldots \ldots$ 3- 1

3.3 COMPLIANCE ASSESSMENT . . . . . . . . . . . . . . 3-2

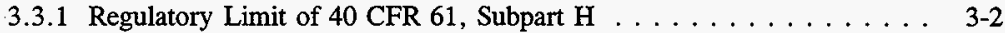

3.3.2 Regulatory Limit of WAC $246-247 \ldots \ldots \ldots \ldots \ldots \ldots \ldots$. . . . . . . .

3.4 METEOROLOGICAL DATA . . . . . . . . . . . . . 3-6

3.5 UNPLANNED RELEASES OF RADIONUCLIDES TO THE ATMOSPHERE 3-6

3.6 ADDITIONAL INFORMATION $\ldots \ldots \ldots \ldots \ldots \ldots$. . . . . . . . .

3.6.1 Construction Projects and Modifications Exempted from 40 CFR 61.96 3-7

3.6.2 Doses to the MEI due to Radon and Thoron Emissions . . . . . . . 3-7

3.6.3 Supplementary Data from Dose Modeling Results . . . . . . . . . 3-8

3.6.4 Revised CY-1996 Release from 296-T-7 Stack . . . . . . . . . . . . 3-10

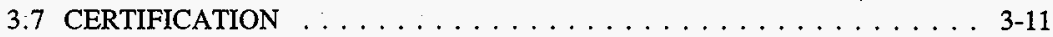

4.0 DIFFUSE AND FUGITIVE SOURCES OF EMISSIONS . . . . . . . . . 4-1

4.1 DIFFUSE AND FUGITIVE EMISSIONS MONITORING $\ldots \ldots \ldots \ldots$. . . .

4.1.1 Near-Facility Environmental Monitoring . . . . . . . . . 4-3

4.1.2 Environmental Surveillance . . . . . . . . . . . . . . 4-5

4.2 ESTIMATED DOSES FROM DIFFUSE AND FUGITIVE EMISSIONS . . 4 4-5

4.2.1 Description of Dose Assessment Method . . . . . . . . . . . . 4-7

4.2.2 Results of Dose Assessment . . . . . . . . . . . . . . . . . . 4-8

4.2.3 Estimate of Uncertainty in Dose Assessment . . . . . . . . . 4-10

4.2.4 Discussion . . . . . . . . . . . . . . . . . . . 4-11

4.3 DIFFUSE AND FUGITIVE EMISSION SOURCES . . . . . . . . . . 4-11

4.3.1 Description of Diffuse and Fugitive Emission Sources . . . . . . . . . 4-12

4.3.2 Description of Specific Diffuse and Fugitive Emission Sources . . . 4 4-18 


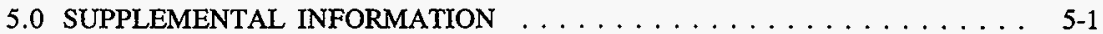

5.1 POPULATION DOSE . . . . . . . . . . . . . . . . 5-1

5.2 COMPLIANCE STATUS WITH 40 CFR 61 , SUBPARTS Q AND T . . . . 5-1

5.3 EMISSION OF ${ }^{220} \mathrm{Rn}$ AND ${ }^{222} \mathrm{Rn}$ FROM TECHNOLOGICALLY ENHANCED SOURCES . . . . . . . . . . . . . . . 5-2

5.4 EMISSIONS ESTIMATES AND CONFIRMATORY MEASUREMENT DATA FOR SPECIFIC NOCs . . . . . . . . . . . . . . . . 5-3

5.4.1 NOC for Portable Temporary Radioactive Airborne Emissions Units

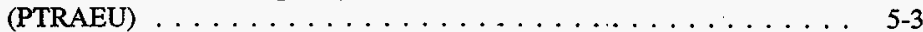

5.4 .2 NOCs for the $309-$ RTF-EX Stack . . . . . . . . . . . . . 5 5-4

5.4.3 Release Estimates for the Containment Tent Exhausters at Tank Farm Facilities . . . . . . . . . . . . . . . . . . 5-4

5.4.4 NOC for the 3732 Process Equipment Development Laboratory Demolition . . . . . . . . . . . . . . . . . . . . . 5-9

5.4.5 NOC for the Excavation and Backfilling Activities in Support of a Potable Water Line Repair . . . . . . . . . . . . . . . . . . . . . . 5 5-10

5.4.6 296-Z-10 Stack Emissions . . . . . . . . . . . . . . . . . . 5-11 5.5 QUALITY ASSURANCE PROGRAMS' COMPLIANCE STATUS . . . . . 5-11

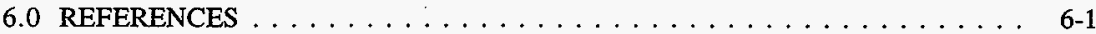

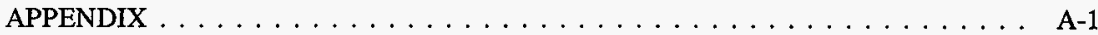




\section{LIST OF FIGURES}

1-1 Hanford Site Map . . . . . . . . . . . . . . . . . . . 1-2

1-2 100-N Area Emission Point Sources $\ldots \ldots \ldots \ldots \ldots \ldots$. . . . . . . . . . . . . . .

1-3 100-K Area Emission Point Sources . . . . . . . . . . . . . . . . . . . 1-8

1-4 200 East Area Emission Point Sources . . . . . . . . . . . . . . . . 1-10

1-5 200 West Area Emission Point Sources . . . . . . . . . . . . . . . . 1-15

1-6 300 Area Emission Point Sources . . . . . . . . . . . . . . . . . . . . . . 1-19

1-7 400 Area Emission Point Sources . . . . . . . . . . . . . . . . . 1-25

3-1 Historical 40 CFR 61, Subpart H Doses to the MEI ... . . . . . . . . 3-3

4-1 Monthly Average Gross Beta Radioactivity in Airborne Particulate Samples, 1979 through $1989 \ldots \ldots$. . . . . . . . . . . . . . . . . . . . 4 4-2

4-2 Near-Facility Ambient Air Sampling Locations . . . . . . . . . . . . . . . 4. 4

4-3 Environmental Surveillance Ambient Air Sampling Locations . . . . . . . . . . 4 4-6

A-1 Meteorological Station Map and Wind Roses for Calendar Year 1997 . . . . . . A-3

A-2 100 Area Wind Rose \& Histogram . . . . . . . . . . . . . . . . . A-4

A-3 200 East Area Wind Rose \& Histogram . . . . . . . . . . . . . . A A 5

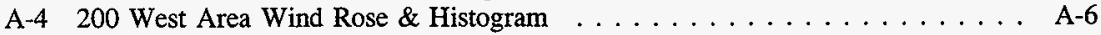

A-5 300 Area Wind Rose \& Histogram . . . . . . . . . . . . . . . . . A A

A-6 400 Area Wind Rose \& Histogram $\ldots \ldots \ldots \ldots \ldots \ldots \ldots$ A-8 . . . . . . . . 


\section{LIST OF TABLES}

2-1 1997 Hanford Site Radionuclide Air Emissions Data for Major Point Sources . . 2-2

2-2 1997 Hanford Site Radionuclide Air Emissions Data for Minor Point Sources . . 2-6

2-3 Distances and Directions from Area Release Locations to an Existing Receptor . 2-14

3-1 Release Estimates of Hanford Site Radionuclide Air Emissions in 1997 . . . . . . 3-4

3-2 1997 CAP88-PC Effective Dose Equivalent Estimates for the Maximally Exposed Individual (at Sagemoor Road) Affected by Radionuclide Air Emissions from the Hanford Site . . . . . . . . . . . . . . . . . . . . . . . . . . 3-5

3-3 1997 Effective Dose Equivalent to MEI (at Sagemoor Road) from 300 Area Radon and Thoron Emissions . . . . . . . . . . . . . . . . . . . . . 3-8

3-4 Doses from Individual Major Point Sources at the Hanford Site in 1997 . . . . . 3-9

3-5 Revised 1996 Releases from the 296-T-7 Stack . . . . . . . . . . . . . . . . . . . 3-10

4-1 Estimated 1997 Hanford Site Diffuse and Fugitive Emissions and Resulting Effective Dose Equivalent . . . . . . . . . . . . . . . . . . . . . . . . 4 4-9

4-2 Types of Waste and Number of Waste Sites at the Hanford Site . . . . . . . . 4 4-14

4-3 Types of Diffuse and Fugitive Emission Sources . . . . . . . . . . . . . . . 4-14

4-4 Hanford Site Soil and Underground Contamination . . . . . . . . . . . . . 4-17

5-1 CY-1997 Emissions Estimates for PTREAUs . . . . . . . . . . . . . . . . 5-3

5-2 CY-1997 Emissions Estimates for the 309-RTF-EX Stack . . . . . . . . . . . 5-4

5-3 1997 Tank Farm Blue Max Use (Location: 244-A) . . . . . . . . . . . . . 5-6

5-4 CY-1997 Ambient Air Monitoring Data Total Alpha and Total Beta Results for the 3732 Process Equipment Development Laboratory Demolition Activities . . . 5-9

5-5 CY-1997 Ambient Air Monitoring Data Composite Sample Results for the 3732 Process Equipment Development Laboratory Demolition Activities . . . 5-10

5-6 CY-1997 Emissions Estimates for the 296-Z-10 Stack . . . . . . . . . . . . . . 5 5-11

A-1 Annual Average Dispersion Factor Around the 100 Area During 1997 for a 10-Meter Release Height . . . . . . . . . . . . . . . . . . . . A-9

A-2 Annual Average Dispersion Factor Around the 200 Areas During 1997 for an 89-Meter Release Height . . . . . . . . . . . . . . . . . . . . A-10

A-3 Annual Average Dispersion Factor Around the 300 Area During 1997 for a 10-Meter Release Height . . . . . . . . . . . . . . . . . . A-11

A-4 Annual Average Dispersion Factor Around the 400 Area During 1997 for a 10-Meter Release Height . . . . . . . . . . . . . . . . . A A-12

A-5 Radionuclide Data Used for CAP88-PC Dose Calculations at Hanford . . . . . A A-13

A-6 Exposure and Consumption Data for the Hanford Site . . . . . . . . . . A A-16

A-7 Hanford Site Meteorological Data - General Site Information . . . . . . . . A-18 
DOE/RL-98-33

\section{LIST OF TERMS}

$\begin{array}{ll}\text { AMU } & \text { aqueous make-up } \\ \text { BHI } & \text { Bechtel Hanford, Incorporated } \\ \text { CAM } & \text { continuous air monitor } \\ \text { CFR } & \text { Code of Federal Regulations } \\ \text { CWC } & \text { Central Waste Complex } \\ \text { D\&D } & \text { decontamination and decommissioning } \\ \text { DCRT } & \text { double-contained receiver tank } \\ \text { DOE } & \text { U.S. Department of Energy } \\ \text { DOE-RL } & \text { U.S. Department of Energy, Richland Operations Office } \\ \text { DST } & \text { double-shell tank } \\ \text { EDE } & \text { effective dose equivalent } \\ \text { EMSL } & \text { 3020 Environmental Molecular Science Laboratory } \\ \text { EPA } & \text { U.S. Environmental Protection Agency } \\ \text { ERC } & \text { Environmental Restoration Contractor } \\ \text { ERDF } & \text { Environmental Restoration Disposal Facility } \\ \text { ETF } & \text { 200 Areas Effluent Treatment Facility } \\ \text { FDH } & \text { Fluor Daniel Hanford, Inc. } \\ \text { FFCA } & \text { Federal Facilities Compliance Agreement } \\ \text { FFTF } & \text { Fast Flux Test Facility } \\ \text { FSS } & \text { Fuel Supply Shutdown } \\ \text { GTF } & \text { Grout Treatment Facility } \\ \text { HEPA } & \text { high-efficiency particulate air (filter) } \\ \text { HT } & \text { elemental tritium } \\ \text { HTO } & \text { tritiated water vapor } \\ \text { HVAC } & \text { heating, ventilation, and air conditioning } \\ \text { MASF } & \text { Maintenance and Storage Facility } \\ \text { MEI } & \text { maximally exposed individual (hypothetical) } \\ \text { MW } & \text { mixed waste } \\ \text { NESHAP } & \text { National Emission Standards for Hazardous Air Pollutants } \\ \text { NPDES } & \text { National Pollutant Discharge Elimination System } \\ \text { NOC } & \text { notice of construction } \\ \text { PFP } & \text { Plutonium Finishing Plant } \\ \text { PHMC } & \text { Project Hanford Management Contract } \\ \text { PNL } & \text { Pacific Northwest Laboratory } \\ \text { PNNL } & \text { Pacific Northwest National Laboratory (formerly PNL) } \\ \text { PRF } & \text { Plutonium Reclamation Facility } \\ \text { PTRAEU } & \text { portable temporary air emission unit } \\ \text { PUREX } & \text { Plutonium-Uranium Extraction (Plant) } \\ \text { RCRA } & \text { Resource Conservation and Recovery Act } \\ \text { REDOX } & \text { Reduction-Oxidation (Plant) } \\ \text { RSB } & \text { Reactor Service Building } \\ \text { SNM } & \text { special nuclear materials } \\ & \end{array}$




\section{LIST OF TERMS (continued)}

SST

Supply System TRIGA

TRU TRUSAF

$\mathrm{UO}_{3}$ Plant

WAC WDOH

WESF

WHC

WIPP

WNP-2

WRAP

WSCF single-shell tank

Washington Public Power Supply System

Test Reactor and Isotope Production by General Atomics transuranic

224-T Transuranic Waste Storage and Assay Facility

Uranium-TriOxide Plant

Washington Administrative Code

State of Washington Department of Health

Waste Encapsulation and Storage Facility

Westinghouse Hanford Company

Waste Isolation Pilot Plant

Washington Nuclear Plant 2

Waste Receiving and Processing Facility

Waste Sampling and Characterization Facility 


\subsection{INTRODUCTION}

\subsection{PURPOSE AND SCOPE}

This report documents radionuclide air emissions from the Hanford Site in 1997, and the resulting effective dose equivalent (EDE) to the maximally exposed member of the public, referred to as the "MEI." The report has. been prepared and will be submitted in accordance with reporting requirements in the Code of Federal Regulations, Title 40, Protection of the Environment, Part 61, National Emissions Standards for Hazardous Air Pollutants, Subpart H, National Emission Standards for Emissions of Radionuclides Other than Radon from Department of Energy Facilities. This report has also been prepared for and will be submitted in accordance with the reporting requirements of the Washington Administrative Code (WAC) Chapter 246-247, Radiation Protection-Air Emissions.

\subsection{SITE DESCRIPTION}

The Hanford Site (Figure 1-1) is located in a rural region of southeastern Washington State between $46^{\circ} 15^{\prime}$ and $46^{\circ} 45^{\prime}$ longitude and between $119^{\circ} 15^{\prime}$ and $120^{\circ} 00^{\prime}$ latitude, occupying an area of about $560 \mathrm{mi}^{2}\left(1,450 \mathrm{~km}^{2}\right)$. It lies about $200 \mathrm{mi}(320 \mathrm{~km})$ northeast of Portland, Oregon; $170 \mathrm{mi}(270 \mathrm{~km})$ southeast of Seattle, Washington; and $124 \mathrm{mi}(200 \mathrm{~km})$ southwest of Spokane, Washington. More in-depth discussions on the Hanford Site's characteristics and activities are available in the Hanford Site National Environmental Policy Act (NEPA) Characterization (PNNL 1997) and the Hanford Site Climatological Summary 1997, with Historical Data (PNNL 1998c).

\subsubsection{Historical Background}

The federal government acquired the Hanford Site in 1943 to construct and operate facilities to produce plutonium for the atomic weapons program during World War II. For more than 40 years, Hanford Site facilities were dedicated primarily to producing plutonium for national defense and managing the wastes generated by chemical processing operations. In more recent years, defense programs have declined considerably while new programs have emerged involving research and development of waste disposal technologies, renewable energy technologies, and cleanup of contamination from past operational practices.

\subsubsection{Major Activities}

Five of the major U.S. Department of Energy (DOE) operating areas existing at the Hanford Site generated radioactive effluents in 1997: the 100, 200 East, 200 West, 300, and 
Figure 1-1. Hanford Site Map.

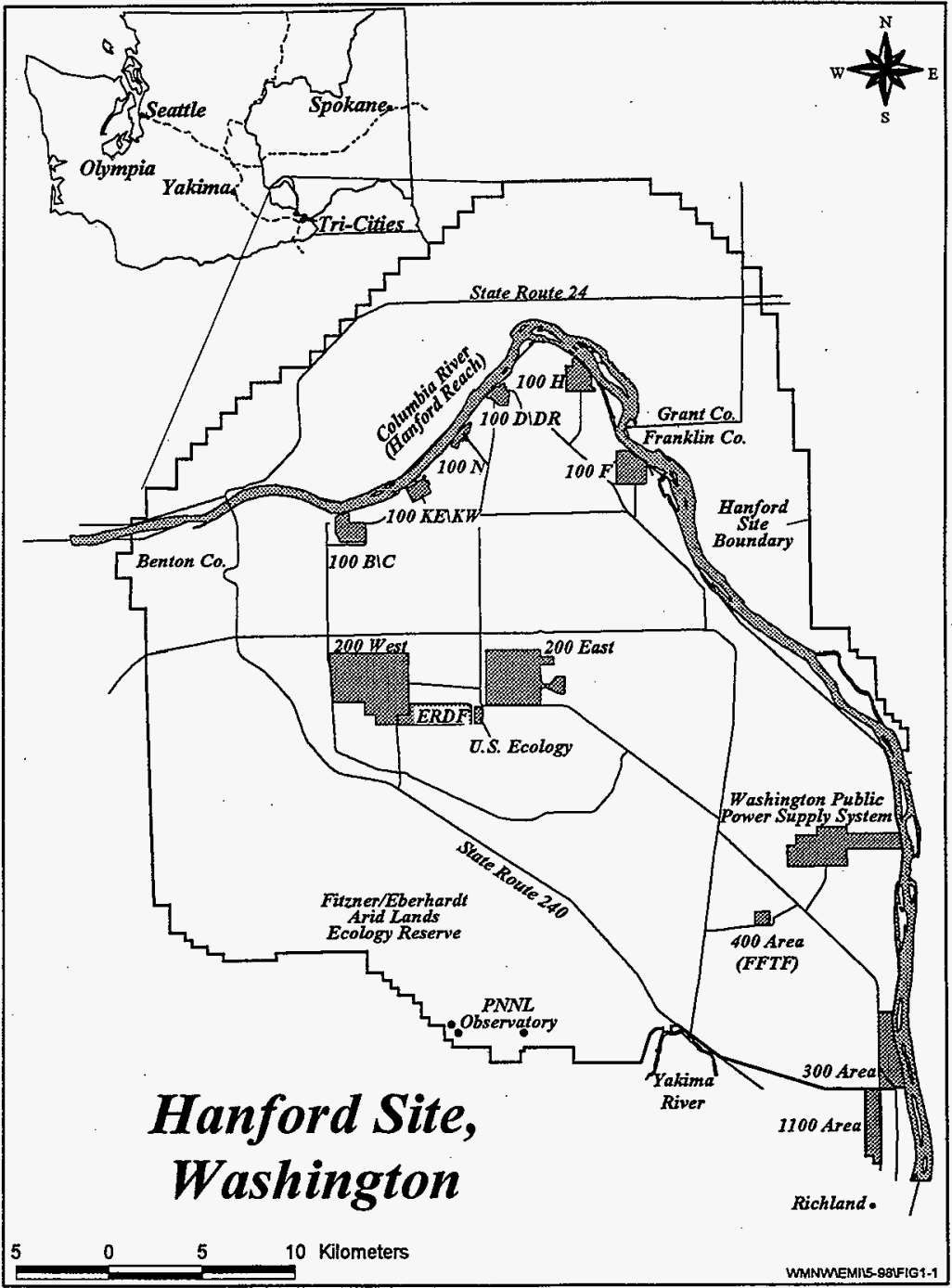


400 Areas (Figure 1-1). The 100 Areas include the $100 \mathrm{~K}$ Spent Fuel Storage Basins, and nine deactivated production reactors, with their support facilities, all located near the Columbia River. The 200 Areas reside on a plateau and are situated about $21.5 \mathrm{mi}$ $(34.7 \mathrm{~km})$ northwest of the city of Richland, Washington and about $7.0 \mathrm{mi}(11.3 \mathrm{~km})$ from the Columbia River. Facilities in the 200 East Area include the PUREX Plant, B Plant, Waste Encapsulation and Storage Facility (WESF), 242-A Evaporator, 200 Areas Effluent Treatment Facility, and East Tank Farms. Facilities in the 200 West Area include the PFP, Uranium-TriOxide ( $\mathrm{UO}_{3}$ ) Plant, West Tank Farms, T Plant, U Plant, REDOX Plant, 222-S Laboratory, waste management units, and Waste Sampling and Characterization Facility (WSCF) immediately outside the 200 West Area fence. The 300 Area, situated approximately $1.0 \mathrm{mi}(1.6 \mathrm{~km})$ due north of the city of Richland, contains research and development laboratories and the deactivated N Reactor Fuel Fabrication Facilities. The 400 Area is the location of the Fast Flux Test Facility (FFTF), approximately $8.0 \mathrm{mi}(12.9 \mathrm{~km})$ north northwest of the city of Richland. The 600 Area includes all facilities not located in the other major operating areas.

Major activities events in 1997, which were relevant to radioactive airborne emissions monitoring and reporting, are summarized as follows.

- Six point sources were deactivated during 1997. Deactivated point sources included: 296-B-5, 296-B-13, 296-Z-10, 309-RTF-EX, EP-331A-01-S and EP-3708-01-S.

- Environmental Restoration activities continued along the river in the 100 and 300 Areas of the Hanford Site. Contaminated soil from inactive waste sites was excavated, transported, and then disposed in the 200 Area Environmental Restoration Disposal Facility (ERDF).

- Cleanup activities at $\mathrm{N}$ Area continued with the deactivation of several facilities, removal and containment of the reactor's radioactive materials, and the removal of radioactively contaminated water. The radioactively contaminated water was transferred to the 200 Areas Effluent Treatment Facility (ETF) and the radioactively contaminated solid waste was disposed to the ERDF.

- Decontamination and Decommissioning of inactive contaminated facilities continued in the $100 \mathrm{~B} / \mathrm{C}$ Area. Interim safe storage activities were initiated at the C Reactor.

- The 3020 Environmental Molecular Science Laboratory (EMSL) began operation in 1997. EMSL is a national focal point for molecular science research with an emphasis on Hanford's long-term environmental management mission.

- ETF operated in 1997, processing mixed aqueous waste streams.

- The 242-A Evaporator operated in 1997, reducing the volume of some of Tank Farm's stored liquid radioactive waste. 
- The 222-S Laboratory operated in 1997 , performing tank waste characterization activities.

- The Waste Sampling and Characterization Facility (WSCF) operated in 1997, analyzing environmental samples.

- PFP Laboratory development testing was conducted on plutonium solutions. Plutonium storage vault activities involved moving considerable quantities of special nuclear material (SNM) to facilitate implementation of International Atomic Energy Agency (IAEA) safeguards on one of the storage vaults. Cleanout activities were also conducted in the 232-Z Incinerator Building.

- At the PUREX Plant, extensive deactivation activities were performed during 1997 and completed by June.

- Extensive deactivation activities were performed at B Plant during 1997.

- WESF continued to safely manage and store the cesium and strontium capsules. WESF's 296-B-10 stack was upgraded to a major point source (potential of $>0.1$ $\mathrm{mrem} / \mathrm{y}$ EDE to nearest offsite receptor) in September 1997. This stack is reported as minor point source for 1997, since it was designated as a minor point source for the majority of 1997.

- $100 \mathrm{~K}$ Spent Fuel Storage Basins continued to store irradiated fuel from N Reactor.

- The N Reactor has not operated since January 1987 and is currently being deactivated prior to being decommissioned. All nuclear fuel has been removed from the reactor core and the major piping systems have been drained.

- The 340 Complex operated in 1997 , shipping liquid waste to the 200 Areas.

- FFTF did not operate in 1997. In December 1993, DOE placed FFTF in transition to a radiologically and industrially safe shutdown condition. FFTF shutdown activities were put on hold in 1996 and remained on hold throughout 1997.

The DOE-RL contractors and their management responsibilities for facilities at the Hanford Site that have radionuclide air emissions are identified as follows.

- Fluor Daniel Hanford, Inc. (FDH): Manages the Project Hanford Management Contract (PHMC), which includes facilities such as N Reactor Fuel Fabrication Facilities, FFTF, Tank Farms, PUREX Plant, B Plant, PFP, 222-S Laboratory, 224-T TRUSAF, WSCF, Central Waste Complex, Low Level Burial Grounds, the 200 Area Power Houses, and the $100 \mathrm{~K}$ Area; conduct liquid effluent monitoring, air emissions monitoring and near-facility environmental surveillance 
at DOE facilities managed by FDH and BHI; provide Hanford Site support services, such as fire protection, central stores, distribution of electrical power, and generation of steam power.

- Battelle Memorial Institute. Manages the Pacific Northwest National Laboratory (PNNL) for DOE, including research and development in the physical, chemical, life, and environmental sciences; produce advanced methods of nuclear waste management; and conduct environmental monitoring on and off the Hanford Site and liquid effluent.and air emission monitoring at the DOE facilities Battelle manages.

- Bechtel Hanford, Incorporated (BHI). BHI is the Environmental Restoration Contractor with responsibility for surveillance and maintenance of inactive pastpractice waste sites and inactive facilities; remediation of past-practice waste sites and contaminated groundwater; closure of Resource Conservation and Recovery Act land-based treatment, storage, and disposal units; overall Hanford Site groundwater project management and sitewide drilling management; and decontamination and decommissioning of facilities.

Privately and publicly owned facilities, capable of generating airborne radioactive emissions, are located at or near the Hanford Site. These facilities include: (1) a low-level radioactive-waste burial site operated by U.S. Ecology, Richland, Washington, on the 200 Area plateau; (2) the Washington Public Power Supply System (Supply System) power reactor (WNP-2) and office buildings, located near the Columbia River, several kilometers north of the 300 Area and east of the 400 Area; (3) the radio-analytical laboratory immediately south of the 300 Area operated by Quanterra, a subsidiary of IT Analytical Services; (4) the Siemens Nuclear Power Corporation fuel fabrication facility, which is immediately adjacent to the southern boundary of the Hanford Site; (5) Allied Technology Group, which is next to Siemens: (6) Interstate Nuclear Services, located $1.6 \mathrm{~km}(1 \mathrm{mi})$ south of Hanford's southern boundary; and (7) Battelle's non-DOE research laboratories in north Richland. Emissions from these privately owned facilities are not included in this report, because these are not regulated as part of the Hanford Site.

\subsection{POINT SOURCE DESCRIPTIONS}

This section includes descriptions for point sources on the Hanford Site. Point sources reported in the certified sections (Sections 1.0, 2.0 and 3.0) of this report met the following criteria during 1997: (1) point source required continuous monitoring or periodic confirmatory measurements by 40 CFR 61 Subpart $H$ or WAC $246-247$, (2) point source was registered with the State of Washington Department of Health (WDOH), (3) the point source normally or potentially had radionuclide air emissions, and (5) the measurement method for the point source is effluent sampling. Point sources not included in the certified sections of this report did not meet these criteria. 
Emissions from the remaining radionuclide air emissions sources are reported in Sections 4.0 and 5.4. Emissions for these sources were estimated using other methods approved by EPA and WDOH.

\subsubsection{General Description and Reporting Criteria}

Radionuclide air emissions from point sources generally are discharged from stacks and vents (further reference to stacks implies vents as well, unless used as the proper name or description of a point source). Stack sizes, shapes, and discharge paths vary because of facility requirements at the time of construction. Discharge heights range from a nearly ground level to $200 \mathrm{ft}(61 \mathrm{~m})$, and flow rates range from less than $100 \mathrm{ft}^{3} / \mathrm{min}\left(0.0472 \mathrm{~m}^{3} / \mathrm{s}\right)$ to more than $200,000 \mathrm{ft}^{3} / \mathrm{min}\left(94.4 \mathrm{~m}^{3} / \mathrm{s}\right)$. Stacks vary in design from horizontal to vertical, rectangular to cylindrical, actively ventilated to passively ventilated, and permanent to portable.

The following methods are used singly or in combination to remove radioactivity from most stack emissions: (1) high-efficiency particulate air (HEPA) filters; (2) sand filters; (3) deep-bed fiberglass filters; (4) fiberglass prefilters; (5) charcoal absorbers; and (6) water scrubbers. Generally, between one and three stages of HEPA filtration are used as the final particle removal method before an emission is exhausted to the atmosphere.

\subsubsection{Areas Facilities}

The 100 Areas contain eight inactive production reactors, the $\mathrm{N}$ Reactor (now in transition to deactivation and decommissioning), and associated support facilities.

Radionuclide air emission points at facilities in the 100-N Area and 100-K Area are described briefly and shown respectively in Figures 1-2 and 1-3.

1.3.2.1 N Reactor. This dual-purpose reactor operated until 1987, producing electrical power for the regional energy system and plutonium for weapons production. The reactor and associated facilities are currently being deactivated before decommissioning.

- 116-N. This stack exhausts filtered air from the 105-N Reactor Building. Emissions sampling consists of a record sampler for particulate radionuclides. In $1993 \mathrm{~N}$ Reactor ventilation was reconfigured such that the 105-N Basin Transfer Area emissions exhaust through the 116-N stack.

- 107-N. This vent exhausts filtered air from the 107-N Basin Recirculation Building. Emissions sampling consists of a record sampler for particulate radionuclides.

- RCF-1-EX. This Radiological Counting Facility (RCF) exhausts filtered air from a hood in the H0-64-4230 trailer. Emissions are periodically sampled for particulate radionuclides. 
Figure 1-2. 100-N Area Emission Point Sources.

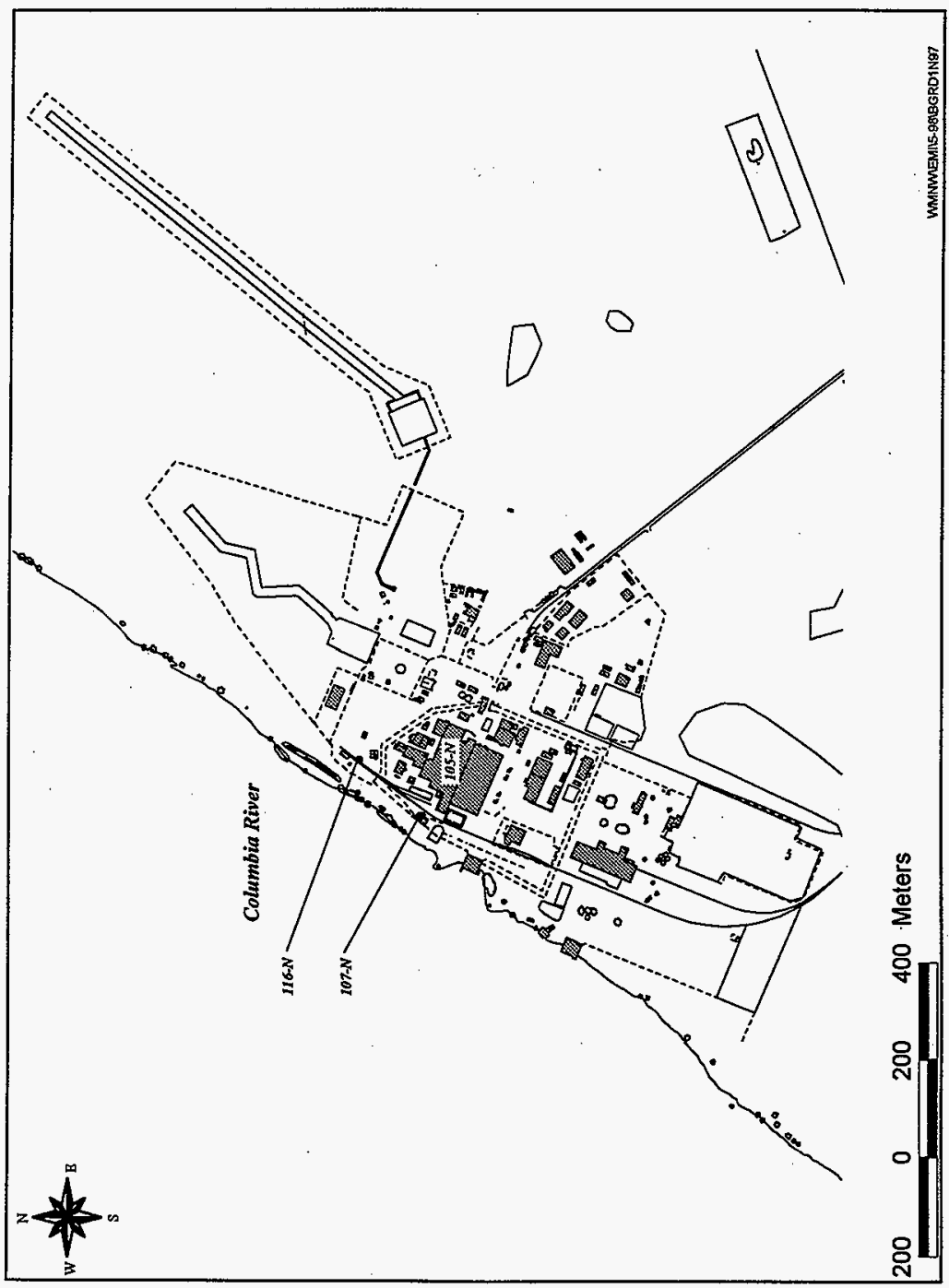


DOE/RL-98-33

Figure 1-3. 100-K Area Emission Point Sources.

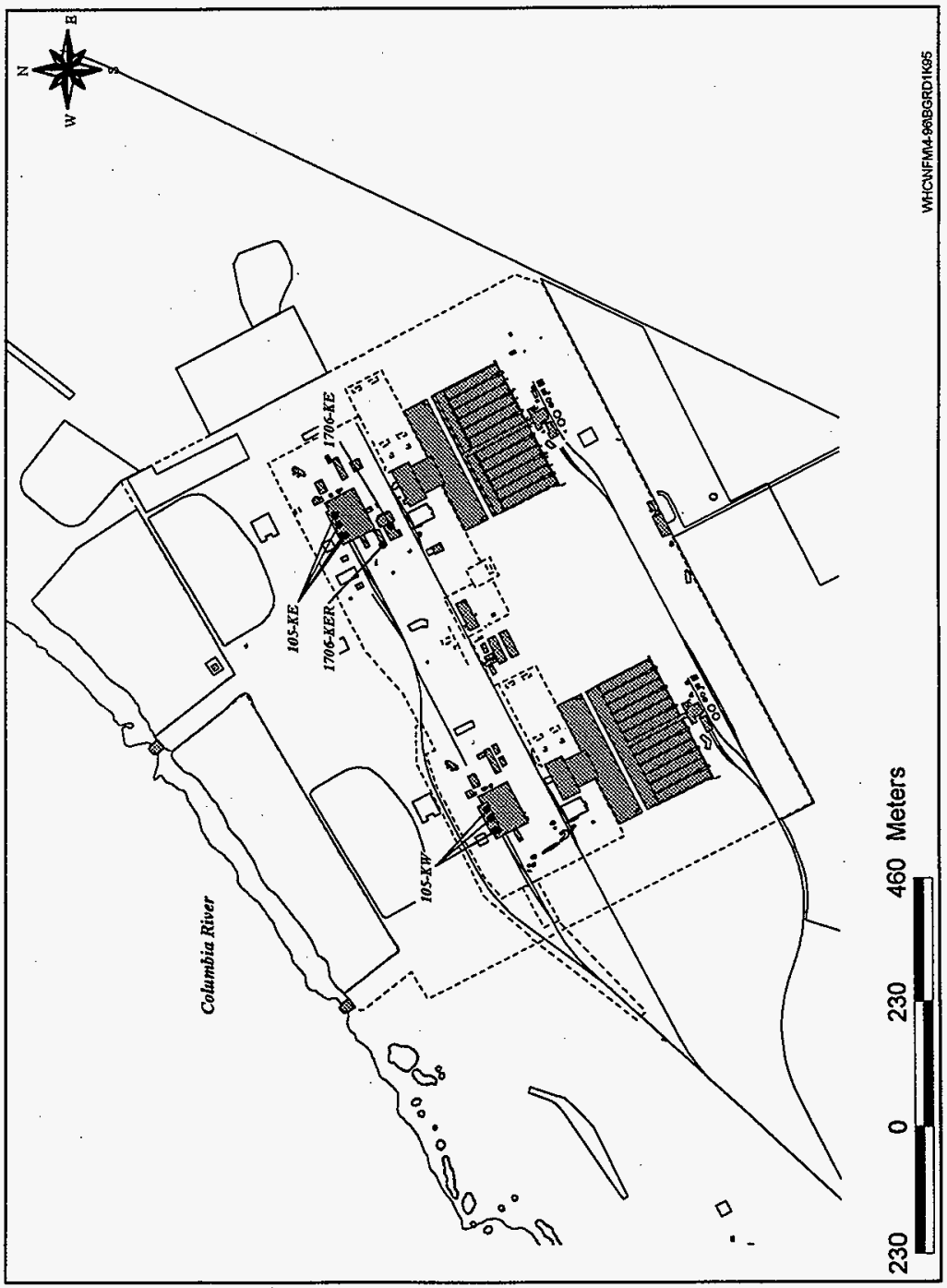


1.3.2.2 100-K East and West Areas. These areas contain two retired reactors awaiting decommissioning, two water-filled storage basins storing irradiated nuclear fuel, and radiological analysis laboratories.

- 105-KE. This point source consists of four powered vents exhausting unfiltered air from the spent fuel storage basin in the 105-KE Building. Emissions sampling consists of four record samplers for particulate radionuclides.

- 105-KW. This point source consists of four powered vents exhausting unfiltered air from the spent fuel storage basin in the 105-KW Building. Emissions sampling consists of three record samplers for particulate radionuclides.

- 1706-KE. This stack exhausts filtered air from the 1706-KE Laboratory. Emissions sampling consists of a record sampler for particulate radionuclides.

- 1706-KER. This stack exhausts filtered air from the basement area (approximately $8.2 \mathrm{~m}$ [ 27 feet] below grade) of the 1706-KE Laboratory. Emissions sampling consists of a record sampler for particulate radionuclides.

\subsubsection{East Area Facilities}

The 200 East Area contains facilities for chemical separations, reprocessing, and waste handling and disposal. Radionuclide air emission discharge points in the 200 East Area are shown in Figure 1-4. The majority of radionuclides discharged from the 200 Areas are in particulate form. The PUREX Plant and related tank farm and evaporator facilities discharge gaseous radionuclides and volatile forms of radionuclides, specifically ${ }^{3} \mathrm{H},{ }^{106} \mathrm{Ru},{ }^{125} \mathrm{Sb}$, and ${ }^{129} \mathrm{I}$. No activities conducted in the 200 Areas in 1997 resulted in a gaseous discharge of ${ }^{85} \mathrm{Kr}$ or ${ }^{13 i} \mathrm{I}$.

1.3.3.1 PUREX Plant. Shutdown orders were issued for the PUREX Plant in December 1992, and as a result, the plant was deactivated by June of 1997. During 1996, deactivation efforts rerouted the PUREX Plant's ventilation, such that all air is discharged through the 291-A-1 stack. As a result of this effort, many of PUREX's stacks were shutdown and deactivated during 1996 and early 1997.

- 291-A-1. This stack exhausts filtered air from canyon ventilation (Cells A to $\mathbf{M}$ ), and vessel and condenser vents. Emissions sampling consists of a record samplers for particulate and volatile radionuclides.

1.3.3.2 B Plant. B Plant was designed and used to separate plutonium from spent nuclear fuel. More recently B Plant was reconfigured to remove ${ }^{137} \mathrm{Cs}$ and ${ }^{90} \mathrm{Sr}$ from high-level liquid waste. The 221-B Building contains radioactive contamination remaining from previous production campaigns that must be properly managed. 


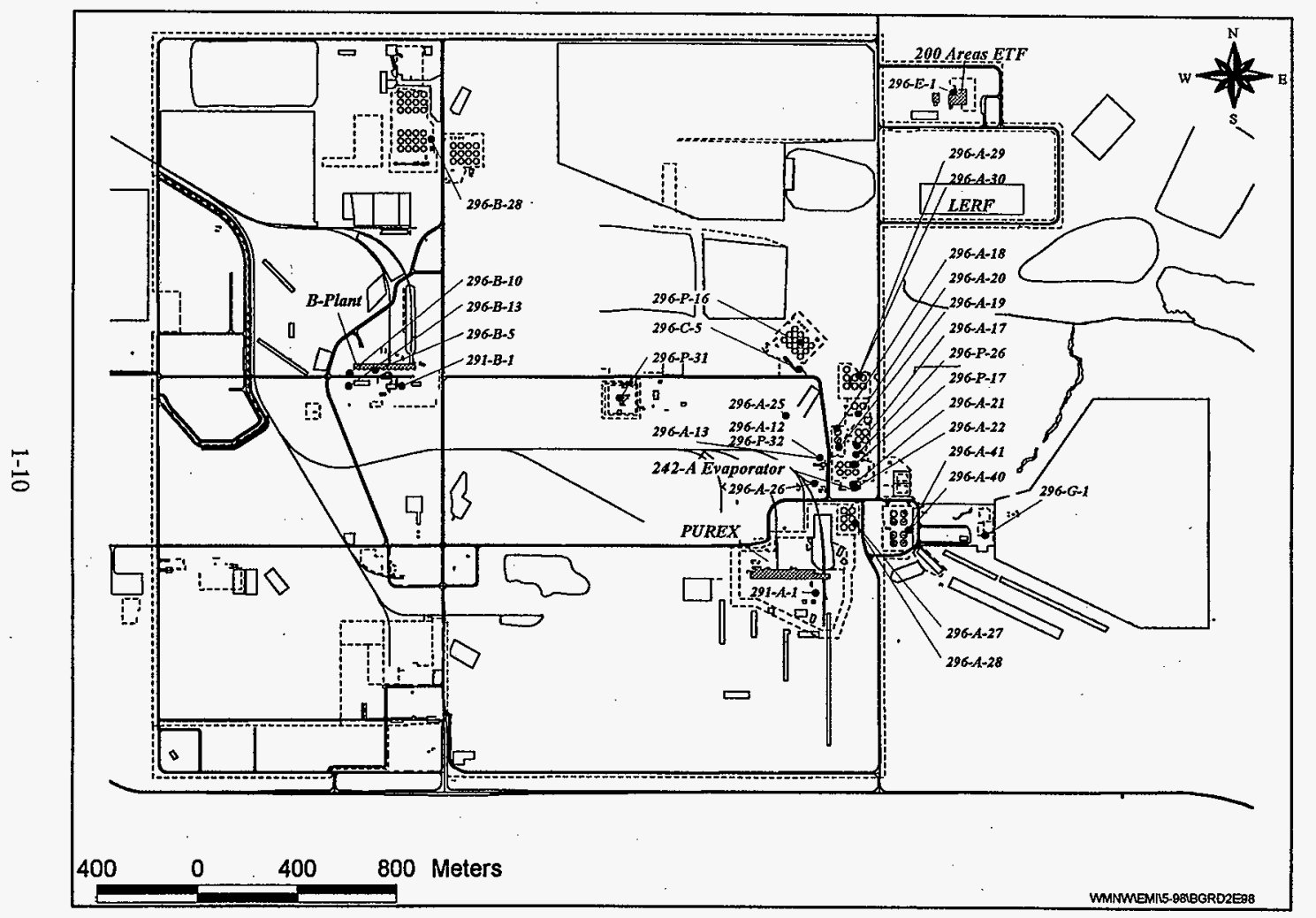

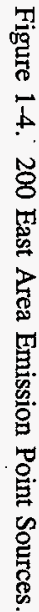


- 291-B-1. This is the B Plant main stack, which exhausts filtered air from the main canyon and process cells in the 221-B Building, from the process cell in the 212-B Building, and the 224-B Building. Emissions sampling consists of a record sampler for particulate radionuclides.

- 296-B-5. This stack exhausted filtered air from the 221-BB Building, which houses the B Plant process condensate and B Plant steam condensate receiver tanks. Emissions sampling consisted of a record sampler, for particulate radionuclides. This stack was permanently shutdown on May 28,1997 and deactivation was completed on May 30, 1997.

- 296-B-13. This stack exhausted filtered air from the 221-BF Condensate Effluent Discharge Facility, which houses process condensate retention tanks. Emissions sampling consisted of a record sampler for particulate radionuclides. This stack was permanently shutdown on February 12, 1997 and deactivation was completed on February 21, 1997.

1.3.3.3 Waste Encapsulation and Storage Facility. WESF was used to convert ${ }^{90} \mathrm{Sr}$ and ${ }^{137} \mathrm{Cs}$, from waste separations, to solid strontium fluoride and cesium chloride. The cesium and strontium were double encapsulated and placed in water-filled storage basins at WESF. WESF's current mission is to store the radioactive strontium and cesium capsules.

- 296-B-10. This stack exhausts filtered air from the 225-B Building. Emissions sampling consists of a record sampler for particulate radionuclides.

1.3.3.4 244-AR and 244-CR Vaults. The 244-AR and 244-CR Vaults are inactive retention facilities for high-level radioactive liquid wastes.

- 296-A-12. This stack exhausts filtered air from the 244-AR Vault vessel ventilation system. Emissions sampling and monitoring consists of a record sampler for particulate radionuclides. This stack did not operate in 1997.

- 296-A-13. This stack exhausts filtered air from the 244-AR Vault canyon and cells. Emissions sampling consists of a record sampler for particulate radionuclides. This stack did not operate in 1997.

- 296-C-5. This stack exhausts filtered air from the 244-CR Vault Cell and vessel ventilation. Emissions sampling consists of a record sampler for particulate radionuclides.

- 296-P-32. During 1997 this stack was connected to exhaust filtered air from the 244-AR Vault vessel ventilation system, since it has better emissions control and sampling systems than the 296-A-12 stack. Emissions sampling consists of a record sampler for particulate radionuclides. In 1997, this stack operated in lieu of the 296-A-12 stack. 
1.3.3.5 200 East Area Tank Farms. Radioactive waste stored in tank farms consists of sludge and saltcake in single-shell tanks (SSTs) and slurry in double-shell tanks (DSTs).

- 296-A-17 and 296-P-26. These stacks exhaust filtered, incondensable vapors from waste storage tanks in the 241-AY and $-\mathrm{AZ}$ Tank Farms. Emissions sampling for each stack consists of a record sampler for particulate and volatile radionuclides. The 296-P-26 stack serves as the backup exhauster for the 296-A-17 stack.

- 296-A-18. This stack exhausts filtered air from the 241-AY-101 tank annulus. Emissions sampling consists of a record sampler for particulate radionuclides.

- 296-A-19. When this stack was operational, filtered air was released through it from the 241-AY-102 tank annulus. Emissions sampling consists of a record sampler for particulate radionuclides.

- 296-A-20. This stack exhausts filtered air from the 241-AZ-101 and -102 tank annuli. Emissions sampling consists of a record sampler for particulate radionuclides. This stack did not operate in 1997.

- 296-A-25. This stack exhausts filtered air from the catch tank at the 244-A lift station. Emissions sampling consists of a record sampler for particulate radionuclides.

- 296-A-26. This stack exhausts filtered air from the waste unloading room and sump tank at the 204-AR tank. Emissions sampling consists of a record sampler for particulate radionuclides.

- 296-A-27. This stack exhausts filtered air from all 241-AW tanks. Emissions sampling consists of a record sampler for particulate and volatile radionuclides.

- 296-A-28. This stack exhausts filtered air from all tank annuli in the 241-AW Tank Farm. Emissions sampling consists of a record sampler for particulate radionuclides.

- 296-A-29. This stack exhausts filtered air from all 241-AN tanks. Emissions sampling consists of a record sampler for particulate radionuclides.

- 296-A-30. This stack exhausts filtered air from all tank annuli in the 241-AN Tank Farm. Emissions sampling consists of a record sampler for particulate radionuclides.

- 296-A-40. This stack exhausts filtered air from all 241-AP tanks. Emissions sampling consists of a record sampler for particulate and volatile radionuclides. 
- 296-A-41. This stack exhausts filtered air from all tank annuli in the 241-AP Tank Farm. Emissions sampling consists of a record sampler for particulate radionuclides.

- 296-B-28. This stack exhausts filtered air from the 244-BX saltwell receiver tank and annulus. Emissions sampling consists of a record sampler for particulate radionuclides.

- 296-P-16. This stack exhausts filtered air from the 241-C-105 and 241-C-106 tanks. The 241-C-104 tank is also vented by this stack as it is connected to the 241-C-105 tank with an underground cascade line. Emissions sampling consists of a record sampler for particulate radionuclides.

- 296-P-33 and 296-P-34. These emission points are the portable exhausters for the Rotary Mode Core Samplers (RMCS). These emission sources exhaust filtered air from the waste tanks being core sampled. Emissions sampling of a record sampler for particulate radionuclides. These stacks did not operate in 1997.

1.3.3.6 242-A Evaporator. The evaporator processed liquid waste in 1997. The 242-A Evaporator is used to remove most of the water from liquid mixed waste, leaving a slurry that is pumped back to tank farms.

- 296-A-21. This stack exhausts filtered air from the 242-A Building. Emissions sampling consists of a record sampler for particulate radionuclides.

- 296-A-22. This stack exhausts filtered air from the 242-A Evaporator vessel ventilation system. Emissions sampling consists of a record sampler for particulate and volatile radionuclides.

1.3.3.7 200 Areas Effluent Treatment Facility. The ETF treats mixed aqueous waste streams prior to their disposal at the 616-A crib.

- 296-E-1. This stack exhausts filtered air from the 2025-E Building and ETF processing vents. Emissions sampling consists of a record sampler for particulate radionuclides.

1.3.3.8 Grout Treatment Facility. This facility solidified low-level waste for disposal in underground vaults. The facility was placed in cold standby in 1993 .

- 296-G-1. When this stack operates air is exhausted from the GTF Air Filtration Module. Emissions sampling consists of a record sampler for particulate radionuclides. This stack did not operate in 1997. 
1.3.3.9 209-E Critical Mass Laboratory. The Building was originally designed and used for testing critical mass configurations. Currently a portion of the building is being used for office space. The laboratory portions of the building are inactive.

- 296-P-31 (formerly 209-E). This stack exhausts filtered building ventilation air. Emissions sampling consists of a record sampler for particulate radionuclides.

\subsubsection{West Area Facilities}

The 200 West Area contains facilities for chemical separations, processing, and waste handling and disposal. The radionuclide air emission discharge points in the 200 West Area are shown in Figure 1-5.

1.3.4.1 REDOX Plant. The Reduction-Oxidation (REDOX) Plant is the 202-S building, which is also known as S Plant. The REDOX Plant was operated as a fuel-reprocessing facility until 1967 when it was shut down.

- 291-S-1. The REDOX main stack exhausts filtered air from the REDOX canyon, vessel ventilation, and treated dissolver offgas system. Emissions sampling consists of a record sampler for particulate radionuclides.

- 296-S-2. This stack exhausts filtered air from REDOX north and south sample galleries, hoods, and product removal cage. Emissions sampling consists of a record sampler, for particulate radionuclides.

- 296-S-7W and 296-S-7E. These stacks exhaust filtered air from the 233-S REDOX product building, the REDOX plutonium-processing greenhouse, and process vessel ventilation and loadout area. Emissions sampling and monitoring for each stack consists of a record sampler for particulate radionuclides. The 296-S-7E stack serves as the backup exhauster for the 296-S-7W.

1.3.4.2 T Plant. $T$ Plant is one of the original fuel reprocessing facilities. The last fuel reprocessed there was in 1956. The 221-T Building is currently used to treat, store, repackage, sample, verify wastes, and decontaminating and repairing equipment.

- 291-T-1. This stack exhausts filtered air from 221- $T$ canyon and process ventilation. Emissions sampling consists of a record sampler for particulate radionuclides.

- 296-T-13. This stack exhausted filtered air from the 221-T Building and canyon ventilation. Emissions sampling consisted of a record sampler for particulate radionuclides. This stack was deactivated in early 1998 , and had not operated since January 1996. 
Figure 1-5. 200 West Area Emission Point Sources.

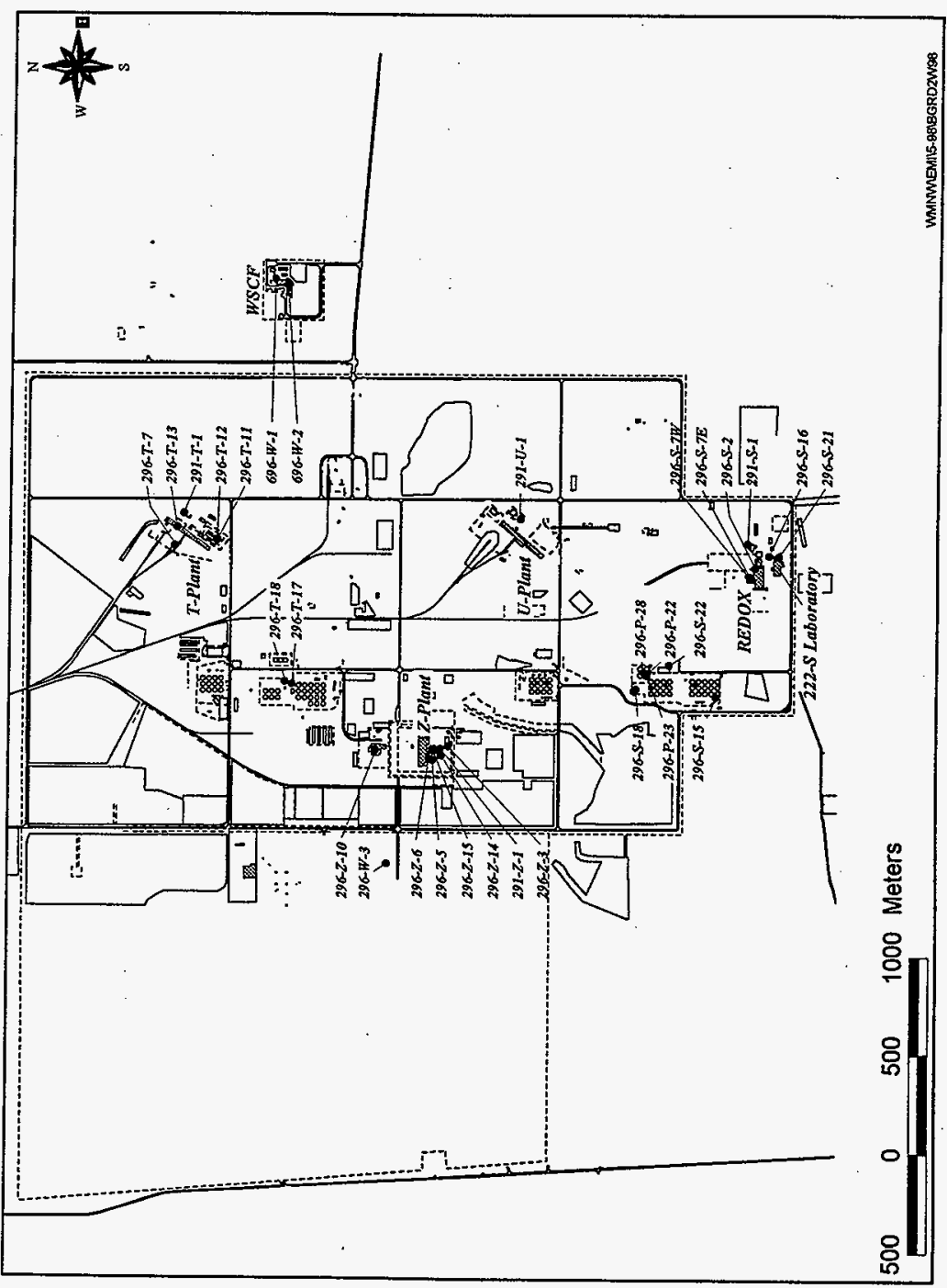


- 296-T-7. This stack exhausts filtered air from the 2706 Building only while decontamination activities are being performed, the remaining times it is turned off. Emissions sampling consists of a record sampler for particulate radionuclides.

1.3.4.3 224-T TRUSAF. The 224-T TRUSAF was originally used to purify plutonium nitrate by the lanthanum fluoride process. After phase-out of the bismuth phosphate plants, the lanthanum fluoride process was no longer needed and the facility remained inactive until the early 1970's. At that time, the 224-T Building was modified for storage of plutonium scrap in liquid and solid forms. In 1984 the facility was selected to house the transuranic waste storage and assay (TRUSAF) operation, in which transuranic waste containers were nondestructively tested and stored for eventual shipment to the Waste Isolation Pilot Plant (WIPP). All stored transuranic wastes were removed from TRUSAF by September 1997, and nondestructive examination and nondestructive assay operations at 224-T TRUSAF were terminated. Currently, no waste is stored and no waste operations are performed at 224-T TRUSAF.

- 296-T-11. This stack exhausts filtered air from the 224-T Building ventilation system through the east part of the plenum. Emissions sampling consists of a record sampler for particulate radionuclides.

- 296-T-12. This stack exhausts filtered air from the 224-T Building ventilation system through the west part of the plenum. Emissions sampling consists of a record sampler for particulate radionuclides.

1.3.4.4 U Plant. U Plant was constructed as a fuel reprocessing plant but was never used for that purpose. Instead, it was used to recover uranium from bismuth-phosphate waste and high-level radioactive wastes from tank farms. Currently, U Plant is a retired facility with a few offices and shops still in use.

- 291-U-1. This stack exhausts filtered air from U Plant and 221-U canyon ventilation. Emissions sampling consists of a record sampler for particulate radionuclides.

1.3.4.5 Plutonium Finishing Plant. PFP was constructed to produce plutonium metal from recovered nitrate and plutonium nitrate received from the PUREX Plant. PFP also recovered plutonium, in the form of plutonium nitrate, from plutonium scrap. PFP's current mission is to stabilize and store existing inventory of plutonium compounds.

- 291-Z-1. This stack exhausts filtered air from the $234-5 Z, 236-Z$, and 242-Z Buildings. Emissions sampling consists of a record sampler for particulate radionuclides.

- 296-Z-3. This stack exhausts filtered air from $241-Z$ vault sump and vessel ventilation. Emissions sampling consists of a record sampler for particulate radionuclides. 
- 296-Z-5. This stack exhausts filtered air from 2736-ZB, the shipping and receiving building. Emissions sampling consists of a record sampler for particulate radionuclides.

- 296-Z-6. This stack exhausts filtered air from the $2736-Z$ Building and its plutonium storage vault ventilation system. Emissions sampling consists of a record sampler for particulate radionuclides.

- 296-Z-14. This stack exhausts filtered air from the $232-Z$ Incinerator Building. Emissions sampling consists of a record sampler for particulate radionuclides.

- 296-Z-15. This stack exhausts filtered air from the 243-Z Low-Level Waste Treatment Facility. Emissions sampling consists of a record sampler for particulate radionuclides.

1.3.4.6 200 West Area Tank Farms. Radioactive waste stored in tank farms consists of sludge and saltcake in SSTs and liquid and slurry in DSTs.

- 296-P-22. This stack exhausts filtered air from annuli in the 241-SY-101, -102, and -103 tanks. Emissions sampling consists of a record sampler for particulate radionuclides.

- 296-P-23 (296-P-28 backup stack). This stack exhausts filtered air from the 241-SY-101, -102, and -103 tanks. Emissions sampling consists of a record sampler for particulate radionuclides.

- 296-S-15. This stack exhausts filtered air from the 241-SX-107 through -112 and 241-SX-114 tanks. Emissions sampling consists of a record sampler for particulate radionuclides.

- 296-S-22. This stack exhausts filtered air from the 244-S saltwell receiver tank and annulus. Emissions sampling consists of a record sampler for particulate radionuclides.

- 296-T-18. This stack exhausts filtered air from the 244-TX saltwell receiver tank and annulus. Emissions sampling consists of a record sampler for particulate radionuclides.

1.3.4.7 200 West Area Evaporators. "The evaporators are designed to remove most of the water from radioactive liquid waste; the resulting slurry is then routed to tank farms for storage. The 242-S Evaporator-Crystallizer did not operate in 1997, but is on standby. The 242-T Evaporator-Crystallizer was deactivated and has been inoperable since 1986.

- 296-S-18. This stack exhausts filtered air from the 242-S EvaporatorCrystallizer Building. Emissions sampling consists of a record sampler for particulate radionuclides. 
296-T-17. This stack exhausts filtered air from the 242-T EvaporatorCrystallizer and cold-cell ventilation. Emissions sampling consists of a record sampler for particulate radionuclides.

1.3.4.8 222-S Laboratory. The 222-S Laboratory provides chemical and radiochemical analytical support for tank farm waste characterization, research and development, and environmental sample analysis.

- 296-S-16. This stack exhausts filtered air from the 219-S Building and waste tanks. Emissions sampling consists of a record sampler for particulate radionuclides.

- 296-S-21. This stack exhausts filtered air from 222-S Laboratory hoods, gloveboxes, hot-cells, and room ventilation system. Emissions sampling consists of a record sampler for particulate radionuclides.

1.3.4.9 Waste Sampling and Characterization Facility. WSCF provides low-level radiological and chemical analyses on various types of samples and sample medias. The majority of the analyzed samples are used to determine compliance with the requirements of environmental regulations and DOE Orders. Note that WSCF is technically located in the 600 Area, and is immediately outside of the 200 West Area's perimeter. WSCF is included in the section for the 200 West Area, since its releases are modelled from the 200 West Area.

- 696-W-1. This stack exhausts filtered air from the analytical laboratory located on the main floor of the 6266 Building. Emissions sampling consists of a record air sampler for particulate radionuclides.

- 696-W-2. This stack exhausts filtered air from the Nuclear Spectroscopy Laboratory (NSL) located in the basement of the 6266 Building. Emissions sampling consists of a record sampler for particulate radionuclides.

1.3.4.10 Waste Verification \& Sampling Facility. The facility is used to verify the contents of drums received from generators. Because of limited use, this facility was transferred to West Tank Farms in 1995.

- 296-W-3. This exhaust discharges filtered air from the 213-W Building.

Emissions sampling consists of a record sampler for particulate radionuclides.

\subsubsection{Area Facilities}

The 300 Area consists primarily of laboratories, research facilities, radioactive liquid waste handling facility, and several inactive facilities associated with prior Hanford missions. Emission points in the 300 Area are shown in Figure 1-6. 
DOE/RL-98-33

Figure 1-6. 300 Area Emission Point Sources.

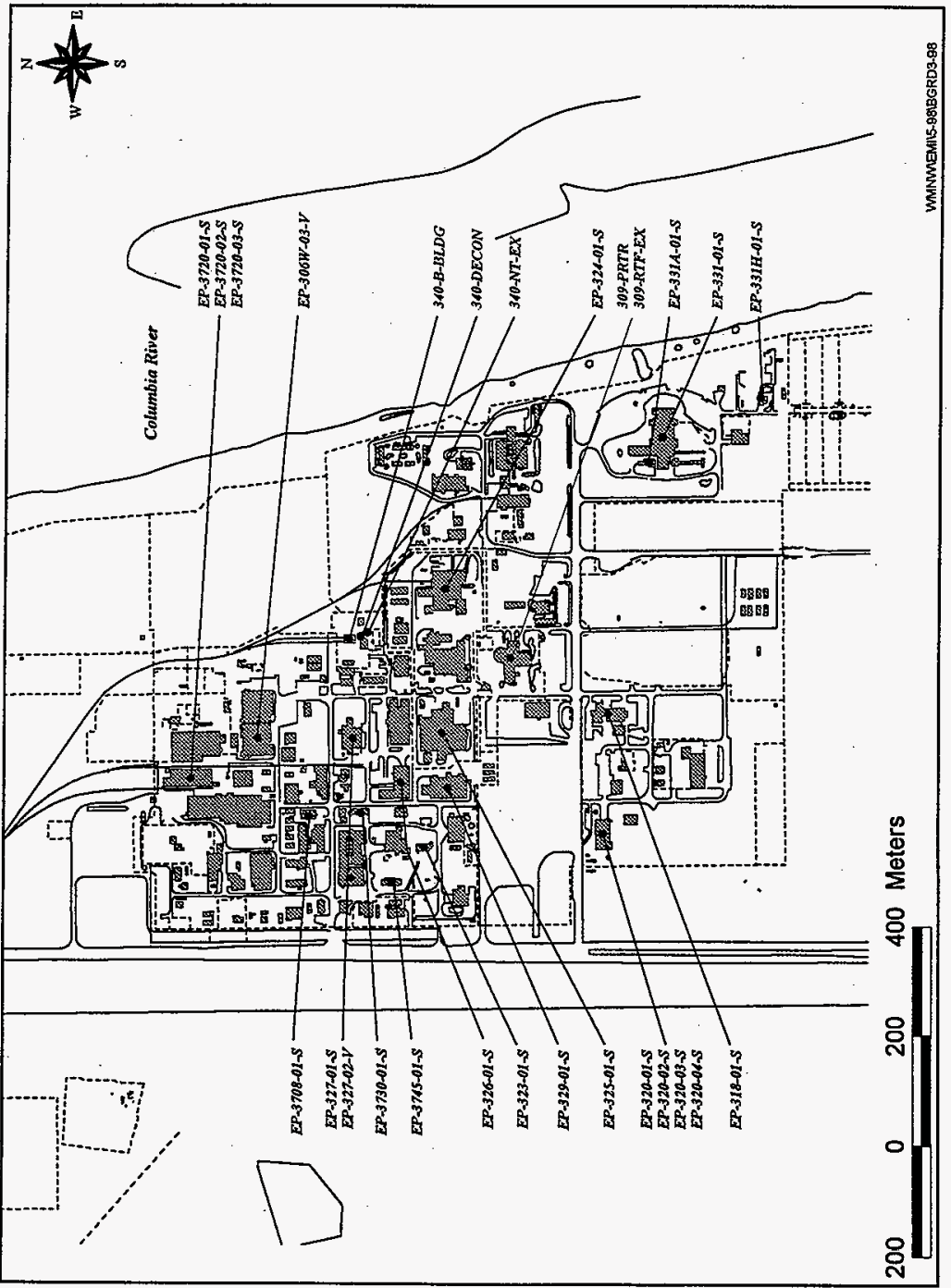


1.3.5.1 305-B Hazardous Waste Storage Building. This building is used to receive, store, and prepare shipments of dangerous waste and radioactive mixed waste generated by Hanford Site research and development programs.

- EP-305B-01-S. This stack exhausts a filtered process hood. The exhaust is sampled for particulate radionuclides.

1.3.5.2 306-W Materials Development Laboratory. The building contains shops and laboratory facilities for metal-working and ceramic studies.

- EP-306W-03-V. This vent exhausts filtered building ventilation air. The exhaust is sampled for particulate radionuclides.

1.3.5.3 309 Plutonium Recycle Test Reactor. The containment dome and support areas of this building once housed the Plutonium Recycle Test Reactor (PRTR). In 1962, the Plutonium Recycle Critical Facility (PRCF) was added to support the PRTR operations. By 1975, the PRTR was deactivated, all of the reactor fuel had been removed from the building, and the fuel storage basin had been decontaminated. In the mid 1980's, an extensive clean out effort removed most of the process equipment and vessels. The ground level of the containment dome is currently being used as an assembly shop and the remainder of the building is used as office space.

- 309-PRTR. This emission point ventilates what used to be the PRTR facility. Emissions are periodically sampled for particulate radionuclides.

1.3.5.4 340 Complex. The 340 Complex houses the radioactive liquid waste and solid waste handling operations for the 300 Area. The 340-A Building contains six aboveground storage tanks for radioactive liquid waste. The east side of the 340-B building is a railway car load out facility for shipping liquid waste to the 200 Areas. The west side of the $340-\mathrm{B}$ building is a storage area for non-radioactive and radioactive solid waste. The 340 Vault house two below grade accumulation tanks for radioactive liquid wastes.

- 340-NT-EX. This stack exhausts filtered air from the 340 Building vault, the 340 Building vault tanks, the 340-A Building waste tanks, and the associated piping system. Emissions sampling consists of a record sampler for particulate volatile radionuclides.

- 340-B-BLDG. This stack exhausts filtered air from the 340-B East Building. The stack exhaust system operates when railway cars are housed within the facility. Emissions sampling consists of a record sampler for particulate radionuclides.

- 340-DECON. This stack exhausts air from the 340 Facility truck lock, operator's office, change rooms, decontamination area, and sampling hood. Emissions sampling consist of a record sampler for particulate radionuclides. 
1.3.5.5 318 Radiological Sciences Laboratory. The building contains areas for calibrating radiation survey instruments and processing personnel dosimeters.

- EP-318-01-S. This stack exhausted unfiltered building ventilation air, until 1998. In early 1998, the emission point was relocated as part of DOE's Energy Savings Performance Contract, and since then it only exhausts a single unfiltered fume hood. Emissions sampling consists of a record sampler for particulate radionuclides.

1.3.5.6 320 Physical Sciences Laboratory. The building contains environmental radiochemistry laboratories.

- EP-320-01-S. This stack exhausts filtered building ventilation air. Emissions sampling consists of a record sampler for particulate radionuclides.

- EP-320-02-S. This stack exhausts filtered chemistry hoods. Emissions sampling consists of a record sampler for particulate radionuclides.

- EP-320-03-S. This stack exhausts filtered chemistry hoods. Emissions sampling consists of a record sampler for particulate radionuclides.

- EP-320-04-S. This stack exhausts filtered chemistry hoods. Emissions sampling consists of a record sampler for particulate radionuclides.

1.3.5.7 323 Mechanical Properties Laboratory. The building contains areas for investigating structural properties of irradiated materials.

- EP-323-01-S. This stack exhausts filtered building ventilation air. Emissions sampling consists of a record sampler for particulate radionuclides.

1.3.5.8 324 Waste Technology Engineering Laboratory. The building contains laboratories for performing chemical and process development activities. The landlord responsibilities for the 324 building were transferred from PNNL to FDH on November 1, 1996.

- EP-324-01-S. This stack exhausts filtered building air. Emissions sampling consists of record samplers for particulate radionuclides and tritium (HTO and HT). 
1.3.5.9 325 Radiochemical Processing Laboratory. The building contains radiochemistry laboratories.

- EP-325-01-S. This stack exhausts filtered building air. Emissions sampling consists of a record sampler for particulate radionuclides and a tritium (HTO and HT) sampler.

1.3.5.10 326 Materials Sciences Laboratory. The building contains laboratories and equipment for studies of metallurgical, chemical, and physical behavior of reactor components and fuel materials.

- EP-326-01-S. This stack exhausts filtered building air. Emissions sampling consists of a record sampler for particulate radionuclides.

1.3.5.11 327 Post Irradiation Testing Laboratory. The building contains hot-cells for examining and testing irradiated materials. The landlord responsibilities for the 327 building were transferred from PNNL to FDH on November 1, 1996.

- EP-327-01-S. This stack exhausts filtered building air. Emissions sampling and monitoring consists of a record sampler for particulate radionuclides, a tritium (HTO and HT) record sampler, and a radon gas monitor.

- EP-327-02-V. This roof vent exhausts filtered air from the decontamination cell. Emissions sampling consists of a record sampler for particulate radionuclides.

1.3.5.12 329 Chemical Sciences Laboratory. The building contains chemistry laboratories for radio-analytical studies, environmental radionuclide studies, and radiation detection instrumentation development.

- EP-329-01-S. This vent exhausts filtered building ventilation air. Emissions sampling consists of a record sampler for particulate radionuclides.

1.3.5.13 331 Life Sciences Laboratory. The building contains areas for biological and ecological research studies.

- EP-331-01-V. This stack exhausts filtered and unfiltered building ventilation air. Emissions sampling consists of a record sampler for particulate radionuclides.

- EP-331A-61-S. This stack exhausted filtered building ventilation air. Emissions sampling consisted of a record sampler for particulate radionuclides. This stack was deactivated on May 30, 1997.

1.3.5.14 331-H Aerosol Wind Tunnel Research Facility. The building contains a wind tunnel for atmospheric pollutant deposition studies. There are no unsealed radionuclides in use in the facility at this time. 
- EP-331H-01-S. This stack exhausted filtered building ventilation air. Emissions sampling consisted of a record sampler for particulate radionuclides. Emissions sampling was not performed for this stack during 1997, since the facility did not contain any unsealed radioactive material. As of 1997, this emission point is no longer considered a radioactive point source.

1.3.5.15 3020 Environmental Molecular Science Laboratory. EMSL contains basic, multidisciplinary research programs involving chemical, biological, materials, and computational sciences. Research and development activities are undertaken at EMSL to advance the understanding of molecular sciences and to apply the information gained to a broad spectrum of environmental restoration and waste management missions. Note that EMSL is technically located outside of the 300 Area, but is near its southern perimeter. It is included in the section for the 300 Area, since its releases are modelled from the 300 Area.

- EP-3020-01-S. This stack exhausts filtered building ventilation air. Emissions sampling consists of a record sampler for particulate radionuclides.

1.3.5.16 3708 Radiation Measurements Laboratory. The building contains areas for measuring small amounts of radionuclides, performing calibration studies, and performing leach studies with vitrified radioactive waste.

- EP-3708-01-S. This stack exhausted filtered building ventilation air. Emissions sampling consisted of a record sampler for particulate radionuclides. This stack was deactivated at the end of 1997.

1.3.5.17 3720 Chemical and Metal Sciences Laboratory. The building contains low-level radiochemistry laboratories and a counting room.

- EP-3720-01-S. This stack exhausts filtered building air. Emissions sampling consists of a record sampler for particulate radionuclides.

- EP-3720-02-S. This stack exhausts filtered building air from the north-annex low-level radiochemistry laboratories. Emissions sampling consists of a record sampler for particulate radionuclides. This stack has not operated since August 1996.

- EP-3720-03-S. This stack exhausts filtered building air from the south-end laboratories. This region of the building contains a counting room and low-level radiochemistry laboratories. Emissions sampling consists of a record sampler for particulate radionuclides.

1.3.5.18 3730 Gamma Irradiation Facility. The building contains a hot-cell for metallurgical studies of specimens of irradiated metals.

- EP-3730-01-S. This stack exhausts filtered building ventilation air. Emissions sampling consists of a record sampler for particulate radionuclides. 
1.3.5.19 3745 Radiological Calibrations and Standards Facility. The building contains a counting room for measuring facility ventilation and exhaust samples.

- EP-3745-01-S. This stack exhausts filtered building ventilation air. Emissions sampling consists of a record sampler for particulate radionuclides.

\subsubsection{Area Facilities}

The 400 Area consists of the Fast Flux Test Facility (FFTF), the Maintenance and Storage Facility (MASF), and the Fuels Materials Examination Facility (FMEF). Emission points in the 400 Area are shown in Figure 1-7.

1.3.6.1 Fast Flux Test Facility. FFTF, located in the 400 Area, is a 400 -megawatt thermal, sodium-cooled, low-pressure, high-temperature reactor plant, that was used for irradiation testing of breeder reactor fuels and materials.

- FFTF-RE-SB. Lower Reactor Service Building (RSB). This exhaust discharges unfiltered air from the lower level of the RSB. Emissions sampling consists of a record sampler for particulate and volatile radionuclides.

- FFTF-CB-EX. Combined exhaust: this stack exhausts normally unfiltered air from the reactor containment and gases from the argon processing system. Standby particulate filters are automatically dampered into the system if airborne radioactive particulate concentrations exceed administrative limits. Emissions sampling consists of an record sampler for particulate and volatile radionuclides, and a tritium (HTO) record sampler.

- FFTF-HT-TR. Heat Transport System South: this exhaust discharges normally unfiltered air from portions of FFTF that are exterior to the containment. Standby particulate filters are automatically dampered into the system if airborne radioactive particulate concentrations exceed administrative limits. Emissions sampling consists of a record sampler for particulate radionuclides.

1.3.6.2 MASF. MASF, 437 Building, is a multipurpose service center supporting the specialized maintenance and storage requirements of FFTF. MASF provides the capability for sodium film removal, decontamination, repair, and storage of nonfueled components and hardware for FFTF.

- 437-MN\&ST. This exhaust discharges filtered air from the MASF. Emissions sampling consists of a record sampler for particulate radionuclides.

- 437-1-61. This exhaust discharges filtered air from the MASF. Emissions sampling consists of a record sampler for particulate radionuclides. 
Figure 1-7. 400 Area Emission Point Sources.

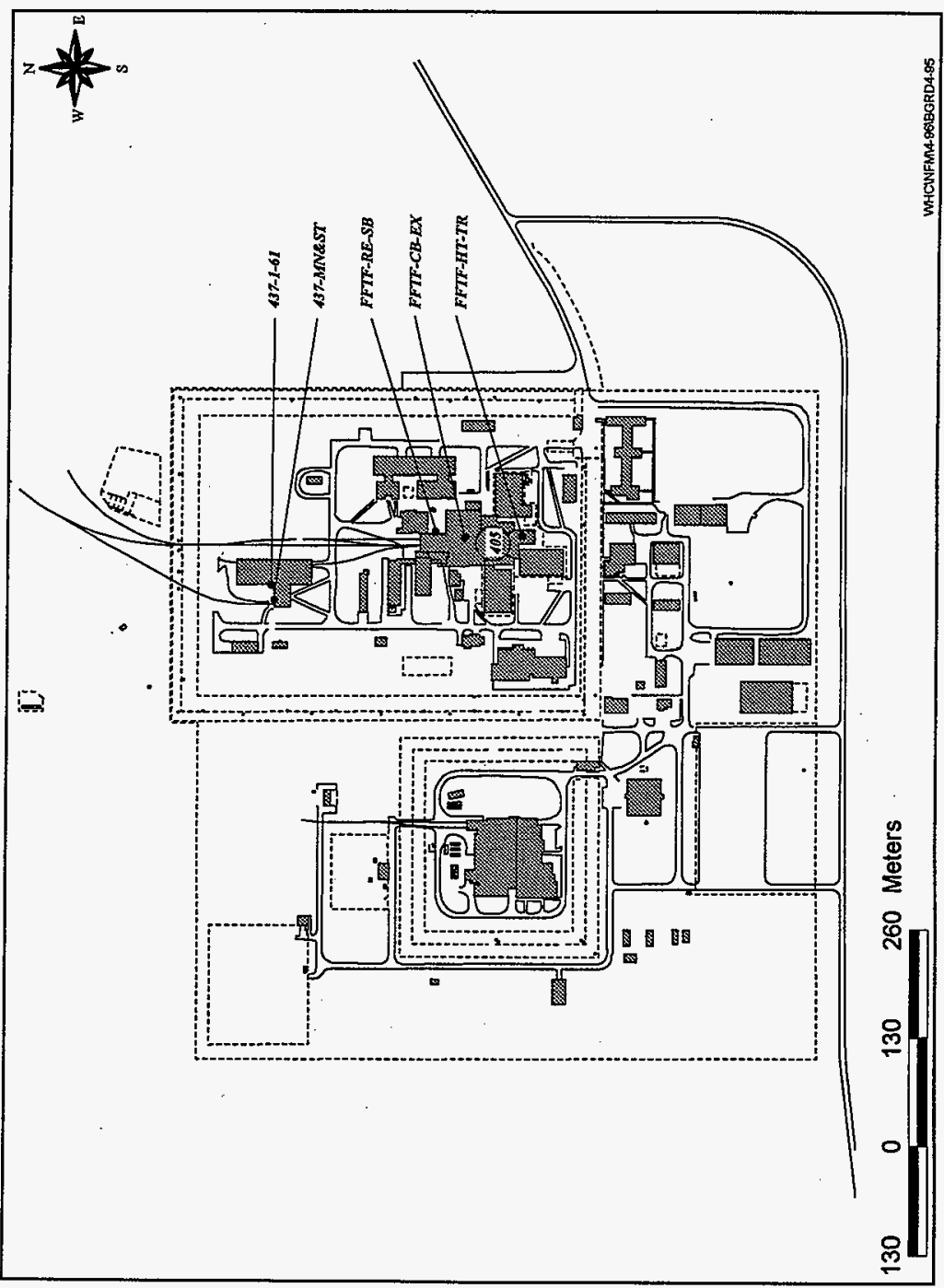


DOE/RL-98-33

This page intentionally left blank. 
DOE/RL-98-33

\subsection{RADIONUCLIDE AIR EMISSIONS DATA ON POINT SOURCES}

The criteria for reporting emissions are discussed in Section 1.3. This section also provides the basis for determining when an emissions source is included in the certified sections (Sections 1.0, 2.0, and 3.0) of this report. Data on radionuclide emissions released during 1997 from actively ventilated point sources (e.g. stacks, vents, etc...), described in Section 1.3.1, are shown in Tables 2-1 and 2-2.

Table 2-1 displays source data on the Hanford Site's 20 major point sources. A point source is classified as major if its emissions, with all treatment controls removed, have the potential to exceed $0.1 \mathrm{mrem} / \mathrm{y}$ effective dose equivalent (EDE), at the nearest receptor.

The dose to a member of the public is evaluated for a known habitable location (i.e., dwelling, business, school, or office) not on the Hanford Site but closest to an emissions area. For the purposes of this report, the emission points on the Hanford Site are grouped by operating area into emissions areas. The maximum exposed member of the public, located at the nearest residence to an operating area, is determined for each emissions area. Thus, five "nearest residence" locations, one for each of the five operating areas, have been established for the Hanford Site. In contrast to the nearest residence locations is the Maximum Exposed Individual (MEI). The MEI is a hypothetical individual, who, by virtue of location and habit, receives the highest dose of any offsite person from the release of radionuclides from all areas on the Hanford Site. In the last several years, the MEI has been calculated to be a resident of a farm east of the 300 Area.

The actively ventilated point sources that do not meet the criteria for a major source are referred to as minor point sources. Table 2-2 displays source data on the Hanford Site's 81 minor point sources. These point sources are grouped according to operating area and facility. A point source is considered minor when its potential maximum emissions with all treatment controls hypothetically removed cannot cause a dose at the nearest residence to exceed $0.1 \mathrm{mrem} / \mathrm{y}$ EDE.

Each point source listed in Tables 2-1 and 2-2 has discharge height, type and efficiency of any emission control, and the 1997 releases by radionuclide or type of radioactivity. The efficiencies for the various emission control devices, used at Hanford, are specified in the footnotes of Tables 2-1 and 2-2.

For dose modelling, the point sources are grouped by operating area, resulting in a single distance applicable to all point sources in that area. Reporting these distances is a requirement of $40 \mathrm{CFR} 61$, Subpart $\mathrm{H}$. The distances and directions to the nearest offsite residences for each emissions area are provided in Table 2-3. Distances to the nearest existing farms producing milk, vegetables, and meat are also included in Table 2-3. Note that the distances provided in Table 3-2 are the distances and directions to the MEI from each emissions area, which are different from those provided in Table 2-3. 
Table 2-1

(4 sheets)

\begin{tabular}{|c|c|c|c|c|c|c|c|}
\hline 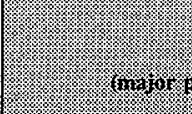 & 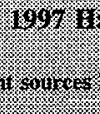 & 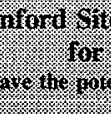 & 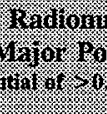 & 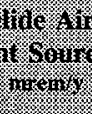 & 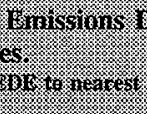 & 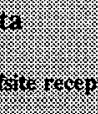 & \\
\hline 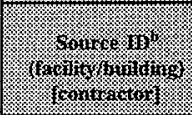 & 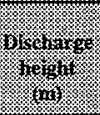 & 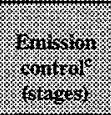 & 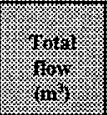 & 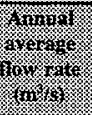 & 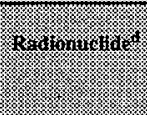 & 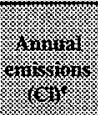 & 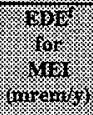 \\
\hline & & & East Area $\mathrm{F}$ & int Source & & & \\
\hline $\begin{array}{l}291-\mathrm{A}-1 \\
\text { (PUREX Plant) } \\
\text { [FDH,BH] }\end{array}$ & 61.0 & HEPA (3) & $7.9 \mathrm{E}+08$ & 25.1 & \begin{tabular}{|l}
${ }^{90} \mathrm{Sr}$ \\
${ }^{106} \mathrm{Ru}$ \\
${ }^{113} \mathrm{Sn}$ \\
${ }^{125} \mathrm{Sb}$ \\
${ }^{129} \mathrm{I}$ \\
${ }^{134} \mathrm{Cs}$ \\
${ }^{137} \mathrm{Cs}$ \\
${ }^{238} \mathrm{Pu}$ \\
${ }^{239.240} \mathrm{Pu}$ \\
${ }^{241} \mathrm{Pu}$ \\
${ }^{241} \mathrm{Am}$
\end{tabular} & $\begin{array}{c}1.5 \mathrm{E}-05 \\
\text { ND } \\
\text { ND } \\
\text { ND } \\
1.2 \mathrm{E}-03 \\
\mathrm{ND} \\
3.1 \mathrm{E}-05 \\
1.5 \mathrm{E}-07 \\
3.1 \mathrm{E}-06 \\
6.4 \mathrm{E}-06 \\
4.2 \mathrm{E}-06\end{array}$ & $\begin{array}{l}3.7 \text { E-07 } \\
2.5 \text { E-04 } \\
1.6 \text { E-06 } \\
2.1 \text { E-07 } \\
4.7 \text { E-06 } \\
1.5 \text { E-07 } \\
9.6 \text { E-06 }\end{array}$ \\
\hline $\begin{array}{l}\text { 291-B-1 } \\
\text { (B Plant) } \\
\text { [FDH] }\end{array}$ & 61.0 & HEPA (2) & $6.1 \mathrm{E}+08$ & 19.3 & $\begin{array}{l}{ }^{90} \mathrm{Sr} \\
{ }^{134} \mathrm{Cs} \\
\cdot{ }^{137} \mathrm{Cs} \\
{ }^{238} \mathrm{Pu} \\
\cdot{ }^{239,240} \mathrm{Pu} \\
{ }^{241} \mathrm{Am}\end{array}$ & $\begin{array}{c}3.9 \mathrm{E}-05 \\
\mathrm{ND} \\
7.9 \mathrm{E}-04 \\
2.6 \mathrm{E}-08 \\
2.9 \mathrm{E}-06 \\
5.7 \mathrm{E}-07\end{array}$ & $\begin{array}{l}9.6 \text { E-07 } \\
4.2 \text { E-05 } \\
3.6 \text { E-08 } \\
4.4 \text { E-06 } \\
1.3 \text { E-06 }\end{array}$ \\
\hline $\begin{array}{l}296-A-22 \\
\text { (242-A Evaporator) } \\
\text { [FDH] }\end{array}$ & $\overline{18.6}$ & HEPA (2) & $6.3 \mathrm{E}+06$ & 0.2 & $\begin{array}{l}{ }^{90} \mathrm{Sr} \\
{ }^{105} \mathrm{Ru} \\
{ }^{113} \mathrm{Sn} \\
{ }^{125} \mathrm{Sb} \\
{ }^{129} \mathrm{~T} \\
{ }^{134} \mathrm{Cs} \\
\cdot{ }^{137} \mathrm{Cs} \\
{ }^{238} \mathrm{Pu} \\
{ }^{239.240} \mathrm{Pu} \\
{ }^{241} \mathrm{Am}\end{array}$ & $\begin{array}{c}3.3 \mathrm{E}-09 \\
\mathrm{ND} \\
\mathrm{ND} \\
\mathrm{ND} \\
\mathrm{ND} \\
\mathrm{ND} \\
\mathrm{ND} \\
\mathrm{ND} \\
8.1 \mathrm{E}-11 \\
4.1 \mathrm{E}-10\end{array}$ & $\begin{array}{c}8.1 \mathrm{E}-11 \\
1.2 \mathrm{E}-10 \\
9.5 \mathrm{E}-10\end{array}$ \\
\hline $\begin{array}{l}\text { 296-A-12 } \\
\text { (TWRS) } \\
\text { [FDH] }\end{array}$ & 45.7 & HEPA (2) & $0.0 \mathrm{E}+00$ & 0.0 & - ${ }^{90} \mathrm{Sr}$ & (did no & perate) \\
\hline $\begin{array}{l}\text { 296-A-17 } \\
\text { 296-P-26 (backup) } \\
\text { (TWRS) } \\
\text { [FDH] }\end{array}$ & 15.2 & HEPA (2) & $4.8 \mathrm{E}+07$ & 1.5 & $\begin{array}{l}{ }^{90} \mathrm{Sr} \\
{ }^{106} \mathrm{Ru} \\
{ }^{113} \mathrm{Sn} \\
{ }^{125} \mathrm{Sb} \\
{ }^{129} \mathrm{I} \\
{ }^{134} \mathrm{Cs} \\
{ }^{137} \mathrm{Cs} \\
{ }^{238} \mathrm{Pu} \\
{ }^{239,240} \mathrm{Pu} \\
{ }^{241} \mathrm{Am}\end{array}$ & $\begin{array}{c}1.6 \mathrm{E}-06 \\
\mathrm{ND} \\
\mathrm{ND} \\
\mathrm{ND} \\
2.1 \mathrm{E}-04 \\
\mathrm{ND} \\
2.0 \mathrm{E}-05 \\
8.8 \mathrm{E}-10 \\
6.9 \mathrm{E}-09 \\
7.5 \mathrm{E}-09\end{array}$ & $\begin{array}{l}3.9 \mathrm{E}-08 \\
4.4 \mathrm{E}-05 \\
1.1 \mathrm{E}-06 \\
1.2 \mathrm{E}-09 \\
1.0 \mathrm{E}-08 \\
1.7 \mathrm{E}-08\end{array}$ \\
\hline
\end{tabular}


Table 2-1

(4 sheets)

\begin{tabular}{|c|c|c|c|c|c|c|c|}
\hline 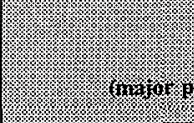 & 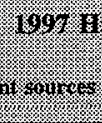 & 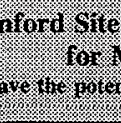 & 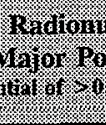 & 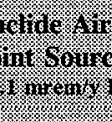 & 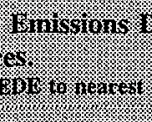 & 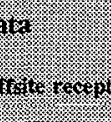 & \\
\hline 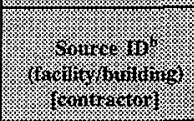 & 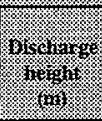 & 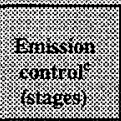 & W.19x & 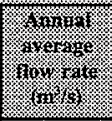 & 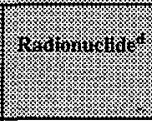 & 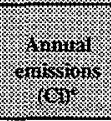 & 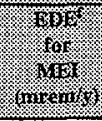 \\
\hline $\begin{array}{l}296-A-25 \\
\text { (TWRS) } \\
\text { [FDH] }\end{array}$ & 3.0 & HEPA (2) & $1.1 E+06$ & $<0.1$ & $\begin{array}{l}{ }^{90} \mathrm{Sr} \\
{ }^{134} \mathrm{Cs} \\
{ }^{137} \mathrm{Cs} \\
{ }^{238} \mathrm{Pu} \\
{ }^{239.240} \mathrm{Pu} \\
{ }^{241} \mathrm{Am}\end{array}$ & $\begin{array}{c}\text { ND } \\
\text { ND } \\
6.4 \mathrm{E}-07 \\
5.7 \mathrm{E}-11 \\
\text { ND } \\
1.4 \mathrm{E}-10\end{array}$ & $\begin{array}{l}3.4 \text { E-08 } \\
8.6 \text { E-11 } \\
3.2 \text { E-10 }\end{array}$ \\
\hline $\begin{array}{l}296-\mathrm{B}-28 \\
\text { (TWRS) } \\
\text { [FDH] }\end{array}$ & 3.4 & HEPA (2) & $3.1 \mathrm{E}+06$ & 0.1 & $\begin{array}{l}{ }^{90} \mathrm{Sr} \\
{ }^{134} \mathrm{Cs} \\
-{ }^{137} \mathrm{Cs} \\
{ }^{238} \mathrm{Pu} \\
{ }^{239.240} \mathrm{Pu} \\
{ }^{241} \mathrm{Am}\end{array}$ & $\begin{array}{c}1.7 \mathrm{E}-09 \\
\mathrm{ND} \\
7.4 \mathrm{E}-10 \\
\mathrm{ND} \\
1.3 \mathrm{E}-10 \\
3.6 \mathrm{E}-10\end{array}$ & $\begin{array}{l}4.2 \mathrm{E}-11 \\
3.9 \mathrm{E}-11 \\
2.0 \mathrm{E}-10 \\
8.3 \mathrm{E}-10\end{array}$ \\
\hline \begin{tabular}{|l|}
$296-C-5$ \\
(TWRS) \\
{$[$ FDH] }
\end{tabular} & 14.6 & HEPA (2) & $4.8 \mathrm{E}+07$ & 1.5 & $\begin{array}{l}\cdot{ }^{96} \mathrm{Sr} \\
{ }^{134} \mathrm{Cs} \\
{ }^{137} \mathrm{Cs} \\
{ }^{238} \mathrm{Pu} \\
{ }^{239,240} \mathrm{Pu} \\
{ }^{241} \mathrm{Am}\end{array}$ & $\begin{array}{c}9.2 \mathrm{E}-08 \\
\mathrm{ND} \\
1.7 \mathrm{E}-07 \\
\text { ND } \\
1.2 \mathrm{E}-09 \\
2.2 \mathrm{E}-09\end{array}$ & $\begin{array}{l}2.3 \mathrm{E}-09 \\
9.0 \mathrm{E}-09 \\
1.8 \mathrm{E}-09 \\
5.1 \mathrm{E}-09\end{array}$ \\
\hline $\begin{array}{l}296-\mathrm{P}-16^{8} \\
\text { (TWRS) } \\
\text { [FDH] }\end{array}$ & 4.6 & HEPA (2) & $4.9 \mathrm{E}+07$ & 1.6 & \begin{tabular}{|l}
${ }^{90} \mathrm{Sr}$ \\
${ }^{134} \mathrm{Cs}$ \\
${ }^{137} \mathrm{Cs}$ \\
${ }^{238} \mathrm{Pu}$ \\
$\cdot{ }^{239.240} \mathrm{Pu}$ \\
${ }^{241} \mathrm{Am}$
\end{tabular} & $\begin{array}{c}1.7 \mathrm{E}-07 \\
\mathrm{ND} \\
4.8 \mathrm{E}-07 \\
1.8 \mathrm{E}-10 \\
4.3 \mathrm{E}-09 \\
4.8 \mathrm{E}-09\end{array}$ & $\begin{array}{ll}4.2 & \mathrm{E}-09 \\
& \\
2.5 & \mathrm{E}-08 \\
2.5 & \mathrm{E}-10 \\
6.5 & \mathrm{E}-09 \\
1.1 & \mathrm{E}-08\end{array}$ \\
\hline $\begin{array}{l}296-\mathrm{P}-32^{\mathrm{h}} \\
\text { (TWRS) } \\
\text { [FDH] }\end{array}$ & 4.6 & HEPA (2) & $1.4 \mathrm{E}+03$ & $<<0.1$ & $\begin{array}{l}{ }^{90} \mathrm{Sr} \\
\text { total alpha } \\
\text { total beta }\end{array}$ & $\begin{array}{c}\mathrm{NM} \\
\mathrm{ND} \\
2.1 \mathrm{E}-09\end{array}$ & $5.2 \mathrm{E}-11$ \\
\hline & & 200 & West Area & Point Source & & & \\
\hline $\begin{array}{l}291-Z-1 \\
\text { (PFP) } \\
{[\text { FDH] }}\end{array}$ & 61.0 & HEPA (1-3) & $4.3 \mathrm{E}+09$ & 137.2 & $\begin{array}{l}{ }^{238} \mathrm{Pu} \\
{ }^{239,240} \mathrm{Pu} \\
{ }^{241} \mathrm{Pu} \\
{ }^{241} \mathrm{Am} \\
\text { total beta }\end{array}$ & $\begin{array}{l}2.2 \mathrm{E}-06 \\
9.3 \mathrm{E}-05 \\
4.6 \mathrm{E}-05 \\
2.0 \mathrm{E}-05 \\
1.2 \mathrm{E}-05\end{array}$ & $\begin{array}{ll}2.4 & \mathrm{E}-06 \\
1.1 & \mathrm{E}-04 \\
8.5 & \mathrm{E}-07 \\
3.6 & \mathrm{E}-05 \\
2.3 & \mathrm{E}-07\end{array}$ \\
\hline $\begin{array}{l}296-S-22 \\
\text { (TWRS) } \\
\text { [FDH] }\end{array}$ & 3.7 & HEPA (2) & $2.1 \mathrm{E}+06$ & 0.1 & $\begin{array}{l}\cdot{ }^{90} \mathrm{Sr} \\
{ }^{134} \mathrm{Cs} \\
\cdot{ }^{137} \mathrm{Cs} \\
{ }^{238} \mathrm{Pu} \\
{ }^{239.240} \mathrm{Pu} \\
\cdot{ }^{241} \mathrm{Am}\end{array}$ & $\begin{array}{c}6.6 \mathrm{E}-09 \\
\text { ND } \\
5.0 \mathrm{E}-10 \\
\text { ND } \\
4.7 \mathrm{E}-11 \\
1.1 \mathrm{E}-10\end{array}$ & $\begin{array}{l}1.3 \text { E-10 } \\
2.1 \text { E-11 } \\
5.5 \text { E-11 } \\
2.0 \text { E-10 }\end{array}$ \\
\hline
\end{tabular}


Table 2-1

(4 sheets)

\begin{tabular}{|c|c|c|c|c|c|c|c|}
\hline (l) & 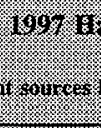 & 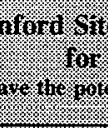 & 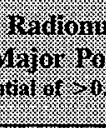 & How & 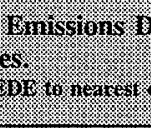 & 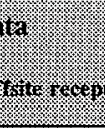 & \\
\hline (1) & mincinising & 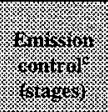 & 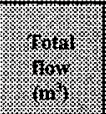 & 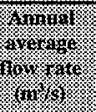 & 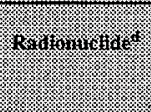 & 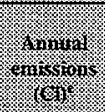 & 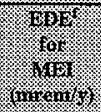 \\
\hline $\begin{array}{l}296-\mathrm{T}-18 \\
\text { (TWRS) } \\
\text { [FDH] }\end{array}$ & 3.7 & HEPA (2) & $3.5 \mathrm{E}+06$ & 0.1 & $\begin{array}{l}{ }^{90} \mathrm{Sr} \\
{ }^{134} \mathrm{Cs} \\
-{ }^{137} \mathrm{Cs} \\
{ }^{238} \mathrm{Pu} \\
\text { - }{ }^{239.240} \mathrm{Pu} \\
{ }^{241} \mathrm{Am}\end{array}$ & $\begin{array}{c}\text { ND } \\
\text { ND } \\
7.2 \text { E-09 } \\
8.6 \text { E-12 } \\
2.6 \text { E-10 } \\
5.1 \text { E-10 }\end{array}$ & $\begin{array}{l}3.0 \text { E-10 } \\
9.3 \text { E-12 } \\
3.0 \text { E-10 } \\
9.1 \text { E-10 }\end{array}$ \\
\hline & & & 00 Area Poi & Sources & & & \\
\hline $\begin{array}{l}\text { 340-NT-EX } \\
\text { (340 Complex) } \\
\text { [FDH] }\end{array}$ & 5.5 & HEPA (2) & $2.6 \mathrm{E}+07$ & 0.8 & $\begin{array}{l}{ }^{131} \mathrm{I} \\
{ }^{137} \mathrm{Cs} \\
{ }^{238} \mathrm{Pu} \\
{ }^{239.240} \mathrm{Pu} \\
{ }^{241} \mathrm{Am} \\
\text { total alpha } \\
\text { total beta }\end{array}$ & $\begin{array}{c}\text { ND } \\
\text { ND } \\
\text { ND } \\
\text { ND } \\
\text { ND } \\
1.2 \mathrm{E}-09 \\
6.9 \mathrm{E}-09\end{array}$ & $\begin{array}{l}8.8 \mathrm{E}-08 \\
6.7 \mathrm{E}-09\end{array}$ \\
\hline $\begin{array}{l}\text { EP-324-01-S } \\
\text { (324 Building) } \\
\text { [FDH] }\end{array}$ & 48.0 & HEPA (2) & $1.0 \mathrm{E}+09$ & 31.7 & $\begin{array}{l}\left.{ }^{3} \mathrm{H} \text { (as HTO) }\right)^{i} \\
{ }^{3} \mathrm{H}(\text { as HT) } \\
\text { - }{ }^{90} \mathrm{Sr} \\
{ }^{137} \mathrm{Cs} \\
{ }^{238} \mathrm{Pu} \\
{ }^{239 / 240} \mathrm{Pu} \\
{ }^{241} \mathrm{Am} \\
\text { total alpha }\end{array}$ & $\begin{array}{l}1.3 \mathrm{E}-01 \\
4.9 \mathrm{E}-01 \\
3.9 \mathrm{E}-08 \\
\text { ND } \\
\text { ND } \\
\text { ND } \\
\text { ND } \\
\text { ND }\end{array}$ & $\begin{array}{l}2.4 \mathrm{E}-05 \\
8.9 \mathrm{E}-07 \\
3.8 \mathrm{E}-08\end{array}$ \\
\hline $\begin{array}{l}\text { EP-325-01-S } \\
\text { (325 Building) } \\
\text { [PNNL] }\end{array}$ & 27.1 & HEPA (2) & $2.0 \mathrm{E}+09$ & 63.4 & $\begin{array}{l}{ }^{3} \mathrm{H} \text { (as HTO) } \\
{ }^{3} \mathrm{H} \text { (as HT) } \\
{ }^{90} \mathrm{Sr} \\
{ }^{137} \mathrm{Cs} \\
\text { - }{ }^{238} \mathrm{Pu} \\
\text { - }{ }^{23927240} \mathrm{Pu} \\
\text { - }{ }^{291} \mathrm{Pu} \\
\text { - }{ }^{241} \mathrm{Am} \\
\quad \text { unsp. alpha } \\
\text { unsp. beta }\end{array}$ & $\begin{array}{c}1.3 \mathrm{E}+00 \\
2.0 \mathrm{E}+01 \\
3.3 \mathrm{E}-08 \\
4.4 \mathrm{E}-08 \\
\mathrm{ND} \\
\mathrm{ND} \\
\mathrm{ND} \\
4.6 \mathrm{E}-09 \\
3.1 \mathrm{E}-08 \\
7.3 \mathrm{E}-08\end{array}$ & $\begin{array}{l}2.4 \mathrm{E}-04 \\
3.6 \mathrm{E}-05 \\
3.2 \mathrm{E}-08 \\
9.1 \mathrm{E}-08 \\
\\
\\
5.2 \mathrm{E}-07 \\
2.3 \mathrm{E}-06 \\
7.1 \mathrm{E}-08\end{array}$ \\
\hline $\begin{array}{l}\text { EP-327-01-S } \\
\text { (327 Building) } \\
\text { [FDH] }\end{array}$ & 14.0 & HEPA (2) & $1.5 \mathrm{E}+09$ & 47.6 & $\begin{array}{l}{ }^{3} \mathrm{H} \text { (as HTO) } \\
{ }^{3} \mathrm{H} \text { (as HT) } \\
{ }^{9} \mathrm{i} \\
{ }^{90} \mathrm{r} \\
{ }^{137} \mathrm{Cs} \\
{ }^{220} \mathrm{Rn}^{j} \\
{ }^{222} \mathrm{Rn} \\
{ }^{233} \mathrm{Pu} \\
{ }^{239.240} \mathrm{Pu} \\
{ }^{241} \mathrm{Am}\end{array}$ & $\begin{array}{c}1.1 \mathrm{E}-01 \\
4.6 \mathrm{E}-02 \\
2.3 \mathrm{E}-07 \\
7.5 \mathrm{E}-07 \\
5.0 \mathrm{E}+01 \\
1.6 \mathrm{E}+00 \\
9.5 \mathrm{E}-10 \\
6.7 \mathrm{E}-09 \\
6.4 \mathrm{E}-09\end{array}$ & $\begin{array}{l}2.0 \mathrm{E}-05 \\
8.3 \mathrm{E}-08 \\
2.2 \mathrm{E}-07 \\
1.5 \mathrm{E}-06 \\
1.9 \mathrm{E}-03 \\
6.4 \mathrm{E}-04 \\
6.4 \mathrm{E}-08 \\
4.9 \mathrm{E}-07 \\
7.2 \mathrm{E}-07\end{array}$ \\
\hline
\end{tabular}


Table 2-1

(4 sheets)

\begin{tabular}{|c|c|c|c|c|c|c|c|}
\hline (3) & 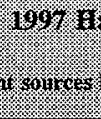 & 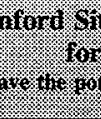 & 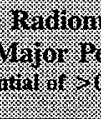 & 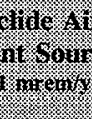 & 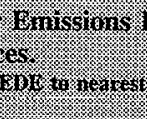 & 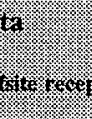 & \\
\hline 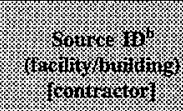 & 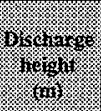 & . & 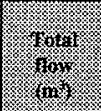 & 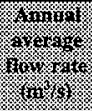 & 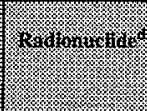 & 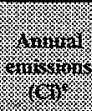 & 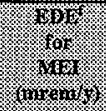 \\
\hline $\begin{array}{l}\text { EP-327-02-V } \\
\text { (327 Building) } \\
\text { [FDH] }\end{array}$ & 9.0 & HEPA (2) & $1.1 \mathrm{E}+07$ & 0.3 & $\begin{array}{l}{ }^{60} \mathrm{Co} \\
{ }^{00} \mathrm{Sr} \\
{ }^{137} \mathrm{Cs} \\
{ }^{238} \mathrm{Pu} \\
{ }^{239,240} \mathrm{Pu} \\
{ }^{241} \mathrm{Am}\end{array}$ & $\begin{array}{c}8.3 \mathrm{E}-10 \\
\mathrm{ND} \\
2.3 \mathrm{E}-09 \\
\mathrm{ND} \\
\mathrm{ND} \\
1.1 \mathrm{E}-11\end{array}$ & $\begin{array}{l}1.8 \text { E-09 } \\
4.7 \text { E-09 } \\
1.2 \text { E-09 }\end{array}$ \\
\hline $\begin{array}{l}\text { EP-3720-01-S } \\
\text { (3720 Building) } \\
\text { [PNNL] }\end{array}$ & 11.0 & HEPA & $3.1 \mathrm{E}+08$ & 9.8 & \begin{tabular}{|l}
${ }^{90} \mathrm{Sr}$ \\
${ }^{137} \mathrm{Cs}$ \\
- ${ }^{238} \mathrm{Pu}$ \\
${ }^{239} \mathrm{P} 240 \mathrm{Pu}$ \\
${ }^{241} \mathrm{Pu}$ \\
${ }^{241} \mathrm{Am}$ \\
unsp. alpha \\
unsp. beta
\end{tabular} & $\begin{array}{c}1.1 \mathrm{E}-08 \\
\mathrm{ND} \\
\mathrm{ND} \\
\mathrm{ND} \\
\mathrm{ND} \\
\mathrm{ND} \\
1.9 \mathrm{E}-08 \\
4.5 \mathrm{E}-08\end{array}$ & $\begin{array}{l}1.4 \mathrm{E}-06 \\
4.4 \mathrm{E}-08\end{array}$ \\
\hline
\end{tabular}

Notes:

a Determining the state of National Emission Standards for Hazardous Air Pollutants (NESHAP) Subpart H compliance for each point source involved using nearest offsite residences, which differed from the MEI.

b ID = Identification, i.e., the alpha-numeric designator for the respective point source; FDH = Fluor Daniel Hanford, Inc.; PNNL = Pacific Northwest National Laboratory (Battelle); BHI = Bechtel Hanford, Inc.

c Efficiencies are: $\geq 99.95 \%$ for HEPA; $\geq 95 \%$ for charcoal; $\geq 99.8 \%$ for sand filter; $0 \%$ for no emission control.

d Builets, "*", identify specific radionuclide sampling and analysis required by 40 CFR 61 Subpart H. "unsp. alpha" and "unsp. beta" stands for unspecified alpha and beta, respectively. Unspecified alpha releases are the result of subtracting the activity released for alpha emitters specifically analyzed for from the total activity released calculated from the total alpha analyses. The same process was used to calculate releases for unspecified beta emitters.

e 1 Curie $=3.7 \mathrm{E}+10$ becquerel; ND $=$ not detected (i.e. either the radionuclide was not detected in any sample during the year, or the average of all the measurements for that given radionuclide or type of radioactivity made during the year were below background levels); $\mathrm{NM}=$ not measured.

f $\mathrm{EDE}=$ Effective dose equivalent for the MEI (at Sagemoor Road farm east of the 300 Area), not the nearest residence; $1 \mathrm{mrem}=1 \mathrm{E}-02 \mathrm{mSv}$.

g It was discovered after the composite analyses were completed that several of the 296-P-16 samples were wet when collected, which jeopardized sample integrity. It could not be determined how the wet samples may have impacted the analytical results.

h The 296-P-32 stack operated for 4 hours CY-1997 during which no samples were collected, since it was not connected to the source term at the.244-AR Vault. A sample collected during January 1998 was used to estimate the CY-1997 releases.

i HTO is tritium as condensable water vapor; HT is tritium as incondensable gas.

j A description of the offsite dose effects of radon emissions is provided in Section 5.3. 
Table 2-2

(8 sheets)

\begin{tabular}{|c|c|c|c|c|c|c|}
\hline 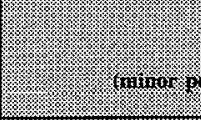 & $39 \mathrm{~m}$ & 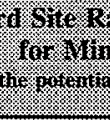 & 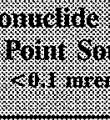 & 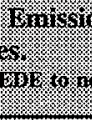 & $\frac{\log }{(x+2)}$ & \\
\hline 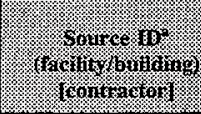 & 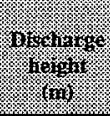 & coninging & 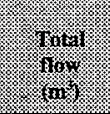 & 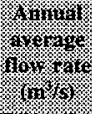 & Rinininime & 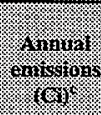 \\
\hline & & $100 \mathrm{~A}$ & Point Sour & & & \\
\hline $\begin{array}{l}116-\mathrm{N} \\
(100 \mathrm{~N} \text { Area) } \\
{[\mathrm{BHI}]}\end{array}$ & 61.3 & $\begin{array}{l}\text { HEPA, } \\
\text { charcoal }\end{array}$ & $1.3 \mathrm{E}+09$ & 41.2 & $\begin{array}{l}{ }^{60} \mathrm{Co} \\
{ }^{90} \mathrm{Sr} \\
{ }^{137} \mathrm{Cs} \\
{ }^{238} \mathrm{Pu} \\
{ }^{239240} \mathrm{Pu} \\
{ }^{241} \mathrm{Am} \\
\text { total alpha } \\
\text { total beta }\end{array}$ & $\begin{array}{c}\text { ND } \\
2.8 \text { E-06 } \\
\text { ND } \\
\text { ND } \\
2.6 \text { E-08 } \\
2.2 \text { E-07 } \\
1.3 \text { E-07 } \\
5.8 \text { E-06 }\end{array}$ \\
\hline $\begin{array}{l}107-\mathrm{N} \\
(100 \mathrm{~N} \text { Area) } \\
{[\mathrm{BHI}]}\end{array}$ & 12.0 & HEPA & $1.1 \mathrm{E}+08$ & 3.5 & $\begin{array}{l}\text { total alpha } \\
\text { total beta }\end{array}$ & $\begin{array}{c}\mathrm{ND} \\
1.8 \mathrm{E}-07\end{array}$ \\
\hline $\begin{array}{l}\text { RCF-1-EX } \\
\text { (100 N Area) } \\
\text { [BHI] }\end{array}$ & 3.0 & HEPA & $2.6 \mathrm{E}+05$ & $<<0.1$ & $\begin{array}{l}\text { total alpha } \\
\text { total beta }\end{array}$ & $\begin{array}{l}4.6 \mathrm{E}-10 \\
8.2 \mathrm{E}-10\end{array}$ \\
\hline $\begin{array}{l}\text { 105-KE Basin } \\
\text { (100 K Area) } \\
\text { [FDH] }\end{array}$ & 12.8 & none & $6.8 \mathrm{E}+08$ & 21.5 & $\begin{array}{l}{ }^{60} \mathrm{Co} \\
{ }^{90} \mathrm{Sr} \\
{ }^{106} \mathrm{Ru} \\
{ }^{125} \mathrm{Sb} \\
{ }^{134} \mathrm{Cs} \\
{ }^{137} \mathrm{Cs} \\
{ }^{154} \mathrm{Eu} \\
{ }^{155} \mathrm{Eu} \\
{ }^{238} \mathrm{Pu} \\
{ }^{2392404} \mathrm{Pu} \\
{ }^{241} \mathrm{Pu} \\
{ }^{241} \mathrm{Am}\end{array}$ & $\begin{array}{c}\text { ND } \\
1.6 \mathrm{E}-05 \\
\text { ND } \\
3.7 \mathrm{E}-09 \\
\mathrm{ND} \\
4.8 \mathrm{E}-05 \\
\mathrm{ND} \\
\mathrm{ND} \\
5.7 \mathrm{E}-07 \\
3.6 \mathrm{E}-06 \\
3.9 \mathrm{E}-05 \\
2.3 \mathrm{E}-06\end{array}$ \\
\hline $\begin{array}{l}\text { I05-KW Basin } \\
\text { (100 K Area) } \\
\text { [FDH] }\end{array}$ & 12.8 & none & $4.2 \mathrm{E}+08$ & 13.3 & $\begin{array}{l}{ }^{60} \mathrm{Co} \\
{ }^{90} \mathrm{Sr} \\
{ }^{106} \mathrm{Ru} \\
{ }^{125} \mathrm{Sb} \\
{ }^{134} \mathrm{Cs} \\
{ }^{137} \mathrm{Cs} \\
{ }^{154} \mathrm{Eu} \\
{ }^{135} \mathrm{Eu} \\
{ }^{238} \mathrm{Pu} \\
{ }^{239240} \mathrm{Pu} \\
{ }^{241} \mathrm{Pu} \\
{ }^{241} \mathrm{Am}\end{array}$ & $\begin{array}{c}2.3 \mathrm{E}-09 \\
8.3 \mathrm{E}-07 \\
\mathrm{ND} \\
\mathrm{ND} \\
\mathrm{ND} \\
7.1 \mathrm{E}-06 \\
\mathrm{ND} \\
\mathrm{ND} \\
6.8 \mathrm{E}-09 \\
3.8 \mathrm{E}-08 \\
6.2 \mathrm{E}-07 \\
4.9 \mathrm{E}-08\end{array}$ \\
\hline $\begin{array}{l}\text { 1706-KER }-27 \mathrm{ft} \\
\text { (100 K Area) } \\
\text { [FDH] }\end{array}$ & 0.9 & HEPA & $1.6 \mathrm{E}+06$ & $<0.1$ & $\begin{array}{l}\text { total alpha } \\
\text { total beta }\end{array}$ & $\begin{array}{l}\text { ND } \\
\text { ND }\end{array}$ \\
\hline
\end{tabular}


Table 2-2

(8 sheets)

\begin{tabular}{|c|c|c|c|c|c|c|}
\hline 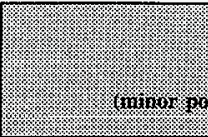 & 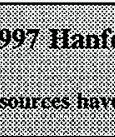 & 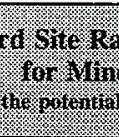 & 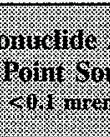 & Q & 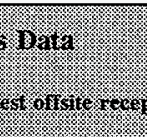 & 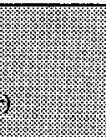 \\
\hline 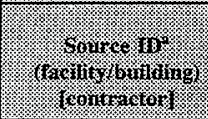 & 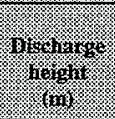 & 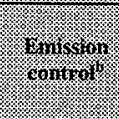 & 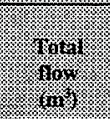 & 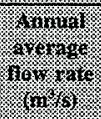 & 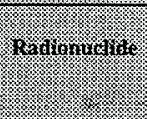 & 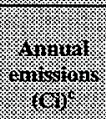 \\
\hline $\begin{array}{l}1706-\mathrm{KE} \\
(100 \mathrm{~K} \text { Area }) \\
{[\mathrm{FDH}]}\end{array}$ & 7.6 & HEPA & $9.1 \mathrm{E}+07$ & 2.9 & $\begin{array}{l}\text { total alpha } \\
\text { total beta }\end{array}$ & $\begin{array}{l}1.9 \text { E-07 } \\
1.1 \text { E-06 }\end{array}$ \\
\hline & & 200 East & ea Point Sol & & & \\
\hline \begin{tabular}{|l|}
$296-\mathrm{B}-5$ \\
(B Plant) \\
[FDH]
\end{tabular} & 3.7 & HEPA & $1.0 \mathrm{E}+07$ & 0.3 & $\begin{array}{l}\text { total alpha } \\
\text { total beta }\end{array}$ & $\begin{array}{l}9.9 \mathrm{E}-09 \\
4.9 \mathrm{E}-08\end{array}$ \\
\hline $\begin{array}{l}\text { 296-B-10 } \\
\text { (B Plant) } \\
\text { [FDH] }\end{array}$ & 22.9 & HEPA & $3.1 \mathrm{E}+08$ & 9.9 & $\begin{array}{l}{ }^{90} \mathrm{Sr} \\
{ }^{134} \mathrm{Cs} \\
{ }^{137} \mathrm{Cs} \\
\text { total alpha }\end{array}$ & $\begin{array}{l}1.8 \mathrm{E}-04 \\
\mathrm{ND} \\
6.1 \mathrm{E}-05 \\
1.3 \mathrm{E}-06\end{array}$ \\
\hline $\begin{array}{l}296-\mathrm{B}-13 \\
\text { (B Plant) } \\
\text { [FDH] }\end{array}$ & 3.5 & HEPA & $1.2 \mathrm{E}+06$ & $<0.1$ & $\begin{array}{l}\text { total alpha } \\
\text { total beta }\end{array}$ & $\begin{array}{l}2.8 \mathrm{E}-09 \\
7.2 \mathrm{E}-09\end{array}$ \\
\hline $\begin{array}{l}296-\mathrm{A}-13 \\
\text { (TWRS) } \\
\text { [FDH] }\end{array}$ & 38.1 & HEPA & $0.0 \mathrm{E}+00$ & 0.0 & (did not & rate) \\
\hline $\begin{array}{l}\text { 296-A-18 } \\
\text { (TWRS) } \\
\text { [FDH] }\end{array}$ & 4.6 & HEPA & $9.6 \mathrm{E}+06$ & 0.3 & $\begin{array}{l}\text { total alpha } \\
\text { total beta }\end{array}$ & $\begin{array}{l}1.2 \mathrm{E}-08 \\
3.7 \mathrm{E}-08\end{array}$ \\
\hline $\begin{array}{l}\text { 296-A-19 } \\
\text { (TWRS) } \\
\text { [FDH] }\end{array}$ & 4.6 & HEPA & $\begin{array}{c}1.6 \mathrm{E}+07 \\
-\end{array}$ & 0.5 & $\begin{array}{l}\text { total alpha } \\
\text { total beta }\end{array}$ & $\begin{array}{l}1.0 \mathrm{E}-08 \\
2.7 \mathrm{E}-08\end{array}$ \\
\hline $\begin{array}{l}\text { 296-A-20 } \\
\text { (TWRS) } \\
{[\text { FDH] }}\end{array}$ & 7.3 & HEPA & $0.0 \mathrm{E}+00$ & 0.0 & (did not & rate) \\
\hline $\begin{array}{l}\text { 296-A-26 } \\
\text { (TWRS) } \\
{[\mathrm{FDH}]}\end{array}$ & 9.4 & HEPA & $2.5 \mathrm{E}+07$ & 0.8 & $\begin{array}{l}\text { total alpha } \\
\text { total beta }\end{array}$ & $\begin{array}{l}8.5 \mathrm{E}-10 \\
4.5 \mathrm{E}-09\end{array}$ \\
\hline $\begin{array}{l}296-A-27 \\
\text { (TWRS) } \\
{[\text { FDH] }}\end{array}$ & 3.7 & HEPA & $1.5 \mathrm{E}+07$ & 0.5 & $\begin{array}{l}\text { total alpha } \\
\text { total beta }\end{array}$ & $\begin{array}{l}3.2 \text { E-09 } \\
1.4 \text { E-07 }\end{array}$ \\
\hline $\begin{array}{l}296-A-28 \\
\text { (TWRS) } \\
{[\text { FDH] }}\end{array}$ & 3.7 & HEPA & $7.8 \mathrm{E}+07$ & 2.5 & $\begin{array}{l}\text { total alpha } \\
\text { total beta }\end{array}$ & $\begin{array}{c}\mathrm{ND} \\
2.2 \mathrm{E}-06\end{array}$ \\
\hline $\begin{array}{l}\text { 296-A-29 } \\
\text { (TWRS) } \\
{[\text { FDH] }}\end{array}$ & 3.7 & HEPA & $1.1 \mathrm{E}+07$ & 0.4 & $\begin{array}{l}\text { total alpha } \\
\text { total beta }\end{array}$ & $\begin{array}{l}9.1 \text { E-10 } \\
6.8 \text { E-07 }\end{array}$ \\
\hline
\end{tabular}


Table 2-2

(8 sheets)

\begin{tabular}{|c|c|c|c|c|c|c|}
\hline (W) & 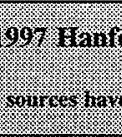 & 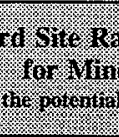 & 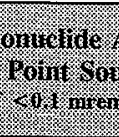 & (6) & 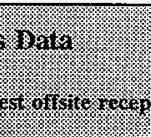 & (3) \\
\hline W & 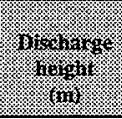 & Hing & 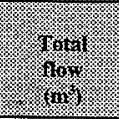 & 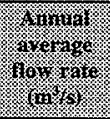 & 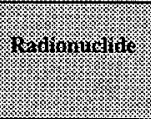 & 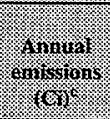 \\
\hline $\begin{array}{l}\text { 296-A-30 } \\
\text { (TWRS) } \\
{[\mathrm{FDH}]}\end{array}$ & 3.7 & HEPA & $7.4 \mathrm{E}+07$ & 2.3 & $\begin{array}{l}\text { total alpha } \\
\text { total beta }\end{array}$ & $\begin{array}{l}5.1 \text { E-08 } \\
2.2 \text { E-07 }\end{array}$ \\
\hline $\begin{array}{l}\text { 296-A-40 } \\
\text { (TWRS) } \\
\text { [FDH] }\end{array}$ & 4.1 & HEPA & $1.4 \mathrm{E}+07$ & 0.4 & $\begin{array}{l}{ }^{90} \mathrm{Sr} \\
{ }^{106} \mathrm{Ru} \\
{ }^{113} \mathrm{Sn} \\
{ }^{125} \mathrm{Sb} \\
{ }^{129} \mathrm{I} \\
{ }^{134} \mathrm{Cs} \\
{ }^{137} \mathrm{Cs} \\
{ }^{238} \mathrm{Pu} \\
{ }^{239.240} \mathrm{Pu} \\
{ }^{241} \mathrm{Am}\end{array}$ & $\begin{array}{c}1.1 \mathrm{E}-09 \\
\text { ND } \\
\text { ND } \\
\text { ND } \\
\text { ND } \\
\text { ND } \\
1.0 \mathrm{E}-09 \\
\text { ND } \\
4.2 \mathrm{E}-10 \\
6.8 \mathrm{E}-10\end{array}$ \\
\hline \begin{tabular}{|l}
$296-A-41$ \\
(TWRS) \\
[FDH]
\end{tabular} & 8.9 & HEPA & $1.2 \mathrm{E}+08$. & 3.9 & $\begin{array}{l}\text { total alpha } \\
\text { total beta }\end{array}$ & $\begin{array}{c}\mathrm{ND} \\
2.6 \mathrm{E}-07\end{array}$ \\
\hline $\begin{array}{l}296-\mathrm{P}-17 \\
\text { (TWRS) } \\
\text { [FDH] }\end{array}$ & 4.6 & HEPA & $0.0 \mathrm{E}+00$ & 0.0 & \multicolumn{2}{|c|}{ (did not operate) } \\
\hline $\begin{array}{l}\text { 296-P-31 } \\
\text { (TWRS) } \\
\text { [FDH] }\end{array}$ & 10.0 & HEPA & $1.6 \mathrm{E}+07$ & 0.5 & $\begin{array}{l}\text { total alpha } \\
\text { total beta }\end{array}$ & $\begin{array}{l}2.9 \mathrm{E}-10 \\
1.3 \mathrm{E}-08\end{array}$ \\
\hline $\begin{array}{l}296-\mathrm{P}-33 \\
296-\mathrm{P}-34 \\
\text { (TWRS) } \\
\text { [FDH] }\end{array}$ & 4.6 & HEPA & $0.0 \mathrm{E}+00$ & 0.0 & \multicolumn{2}{|c|}{ (did not operate) } \\
\hline $\begin{array}{l}\text { 296-A-21 } \\
\text { (242-A Evaporator) } \\
\text { [FDH] }\end{array}$ & 6.7 & HEPA & $1.8 \mathrm{E}+08$ & 5.7 & $\begin{array}{l}\text { total alpha } \\
\text { total beta }\end{array}$ & $\begin{array}{l}3.6 \mathrm{E}-08 \\
4.0 \mathrm{E}-06\end{array}$ \\
\hline $\begin{array}{l}296-\mathrm{E}-1 \\
\text { (ETF) } \\
{[\mathrm{FDH}]}\end{array}$ & 15.5 & HEPA & $8.2 E+08$ & 26.0 & $\begin{array}{l}\text { total alpha } \\
\text { total beta }\end{array}$ & $\begin{array}{l}1.1 \mathrm{E}-07 \\
4.1 \mathrm{E}-07\end{array}$ \\
\hline $\begin{array}{l}296-G-1 \\
\text { (Grout) } \\
\text { [FDH] }\end{array}$ & 7.6 & HEPA & $0.0 \mathrm{E}+00$ & 0.0 & \multicolumn{2}{|c|}{ (did not operate) } \\
\hline \multicolumn{7}{|c|}{200 West Area Point Sources } \\
\hline $\begin{array}{l}\text { 296-P-22 } \\
\text { (TWRS) } \\
\text { [FDH] }\end{array}$ & 4.6 & HEPA & $1.3 \mathrm{E}+07$ & 0.4 & $\begin{array}{l}\text { total alpha } \\
\text { total beta }\end{array}$ & $\begin{array}{l}4.4 \text { E-09 } \\
6.1 \text { E-08 }\end{array}$ \\
\hline
\end{tabular}


Table 2-2

(8 sheets)

\begin{tabular}{|c|c|c|c|c|c|c|}
\hline 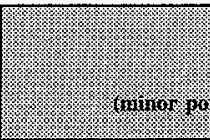 & Mrom & 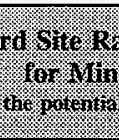 & 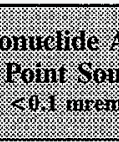 & Thinging & 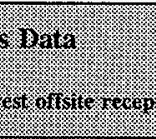 & \\
\hline 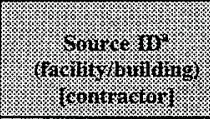 & 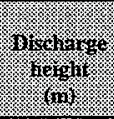 & 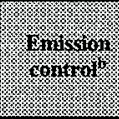 & 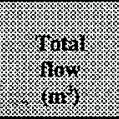 & 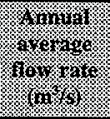 & 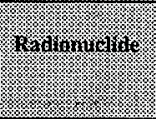 & 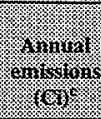 \\
\hline $\begin{array}{l}296-P-23 \\
296-P-28 \text { (backup) } \\
\text { (TWRS) } \\
\text { [FDH] }\end{array}$ & 4.6 & HEPA & $1.3 \mathrm{E}+07$ & 0.4 & $\begin{array}{l}\text { total alpha } \\
\text { total beta }\end{array}$ & $\begin{array}{l}2.7 \mathrm{E}-09 \\
1.1 \mathrm{E}-06\end{array}$ \\
\hline $\begin{array}{l}296-S-15 \\
\text { (TWRS) } \\
\text { [FDH] }\end{array}$ & 4.6 & HEPA & $5.1 \mathrm{E}+07$ & 1.6 & $\begin{array}{l}\text { total alpha } \\
\text { total beta }\end{array}$ & $\begin{array}{l}9.0 \mathrm{E}-09 \\
4.4 \mathrm{E}-07\end{array}$ \\
\hline $\begin{array}{l}\text { 296-S-18 } \\
\text { (TWRS) } \\
\text { [FDH] }\end{array}$ & 6.7 & HEPA & $1.1 \mathrm{E}+08$ & 3.4 & $\begin{array}{l}\text { total alpha } \\
\text { total beta }\end{array}$ & $\begin{array}{ll}1.1 \mathrm{E}-07 \\
4.1 \mathrm{E}-07\end{array}$ \\
\hline $\begin{array}{l}296-\mathrm{T}-17 \\
\text { (TWRS) } \\
{[\mathrm{FDH}]}\end{array}$ & 10.1 & HEPA & $1.7 \mathrm{E}+07$ & 0.5 & $\begin{array}{l}\text { total alpha } \\
\text { total beta }\end{array}$ & $\begin{array}{c}\mathrm{ND} \\
3.4 \mathrm{E}-08\end{array}$ \\
\hline $\begin{array}{l}296-W-3 \\
\text { (TWRS) } \\
{[\mathrm{FDH}]}\end{array}$ & 7.6 & HEPA & $4.9 \mathrm{E}+06$ & 0.2 & $\begin{array}{l}\text { total alpha } \\
\text { total beta }\end{array}$ & $\begin{array}{ll}3.2 & \text { E-08 } \\
4.5 & \text { E-08 }\end{array}$ \\
\hline $\begin{array}{l}\text { 291-S-1 } \\
\text { (REDOX Plant) } \\
\text { [BHI] }\end{array}$ & 61.0 & sand filter & $3.0 \mathrm{E}+08$ & 9.5 & $\begin{array}{l}\text { total alpha } \\
\text { total beta }\end{array}$ & $\begin{array}{c}\text { ND } \\
4.8 \mathrm{E}-06\end{array}$ \\
\hline $\begin{array}{l}296-\mathrm{S}-2 \\
\text { (REDOX Plant) } \\
\text { [BHI] }\end{array}$ & 20.7 & HEPA & $1.1 \mathrm{E}+07$ & 0.3 & $\begin{array}{l}\text { total aipha } \\
\text { total beta }\end{array}$ & $\begin{array}{l}3.3 \mathrm{E}-08 \\
6.9 \mathrm{E}-08\end{array}$ \\
\hline $\begin{array}{l}296-S-7 W \\
\text { 296-S-7E (backup) } \\
\text { (REDOX Plant) } \\
\text { [BHI] }\end{array}$ & 7.6 & HEPA & $1.2 \mathrm{E}+08$ & 3.8 & $\begin{array}{l}\text { total alpha } \\
\text { total beta }\end{array}$ & $\begin{array}{l}3.2 \mathrm{E}-06 \\
7.4 \mathrm{E}-06\end{array}$ \\
\hline $\begin{array}{l}291-\text { U-I } \\
\text { (U Plant) } \\
{[\mathrm{BHI}]}\end{array}$ & 61.0 & sand filter & $3.9 \mathrm{E}+08$ & 12.4 & $\begin{array}{l}\text { total alpha } \\
\text { total beta }\end{array}$ & $\begin{array}{l}8.1 \text { E-07 } \\
2.0 \text { E-04 }\end{array}$ \\
\hline $\begin{array}{l}291-\mathrm{T}-1 \\
\text { (T Plant) } \\
{[\mathrm{FDH}]}\end{array}$ & 61.0 & HEPA & $4.8 \mathrm{E}+08$ & 15.2 & $\begin{array}{l}\text { total alpha } \\
\text { total beta }\end{array}$ & $\begin{array}{l}1.0 \mathrm{E}-05 \\
7.5 \mathrm{E}-05\end{array}$ \\
\hline $\begin{array}{l}296-\mathrm{T}-7 \\
\text { (T Plant) } \\
\text { [FDH] }\end{array}$ & 8.5 & HEPA & $4.4 \mathrm{E}+07$ & 1.4 & $\begin{array}{l}\text { total alpha } \\
\text { total beta }\end{array}$ & $\begin{array}{l}1.1 \mathrm{E}-08 \\
3.3 \mathrm{E}-08\end{array}$ \\
\hline $\begin{array}{l}296-T-13 \\
\text { (T Plant) } \\
\text { [FDH] }\end{array}$ & 20.7 & HEPA & $0.0 \mathrm{E}+00$ & 0.0 & \multicolumn{2}{|c|}{ (did not operate) } \\
\hline
\end{tabular}


Table 2-2

(8 sheets)

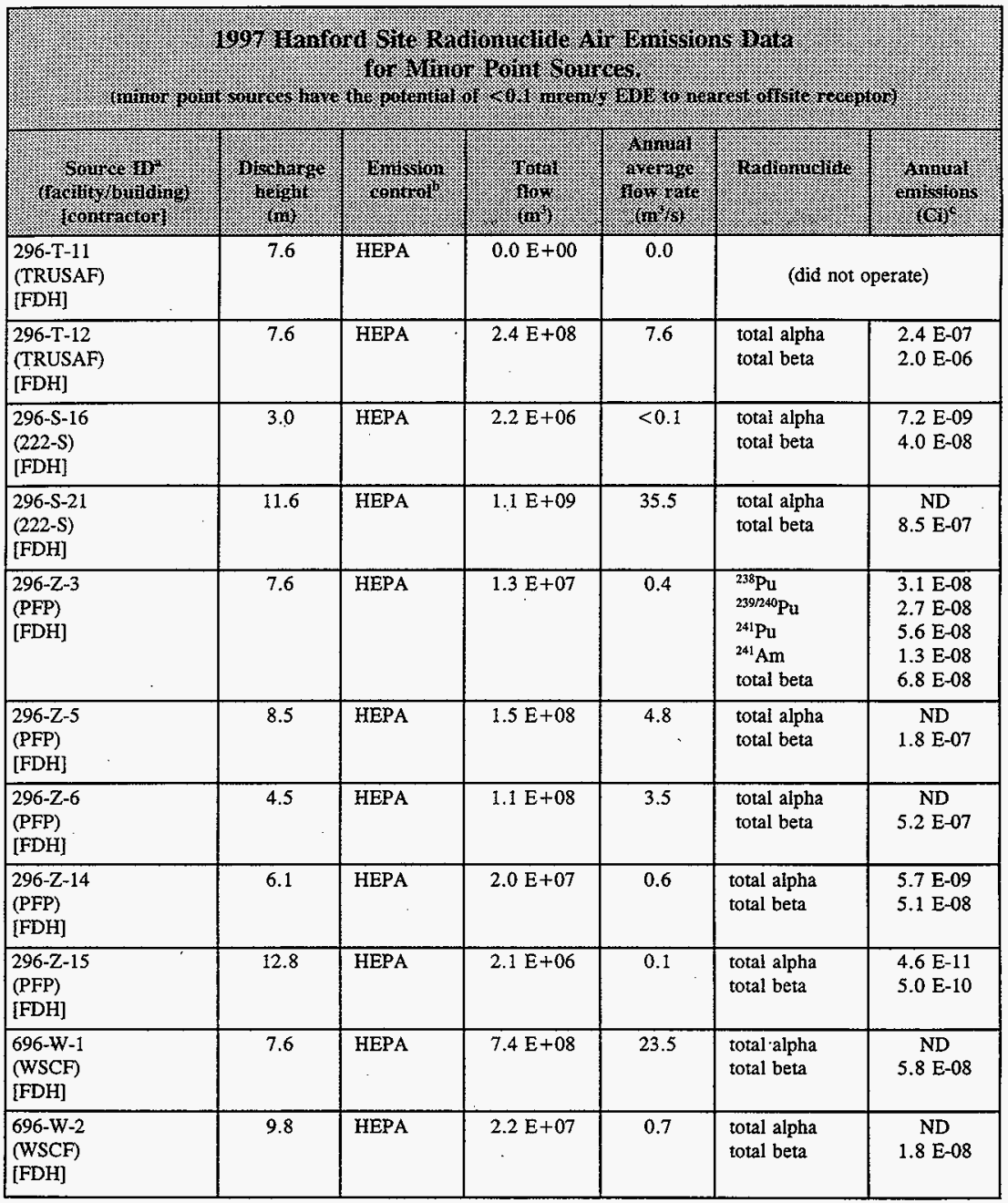


Table 2-2

(8 sheets)

\begin{tabular}{|c|c|c|c|c|c|c|}
\hline 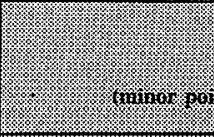 & 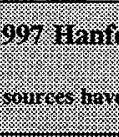 & 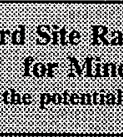 & 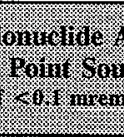 & 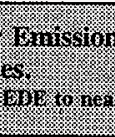 & 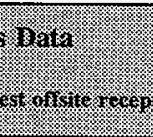 & $y_{1}$ \\
\hline (6) & 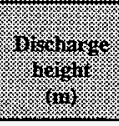 & 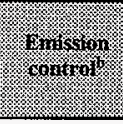 & 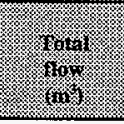 & 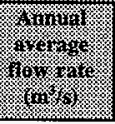 & 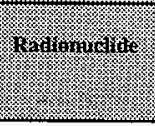 & 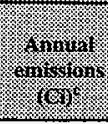 \\
\hline & & $300 \mathrm{~A}$ & Point Source & & & \\
\hline $\begin{array}{l}\text { 309-PRTR } \\
\text { (309 Building) } \\
{[\mathrm{FDH}]}\end{array}$ & 30.5 & HEPA & $7.2 E+07$ & 2.3 & $\begin{array}{l}\text { total aipha } \\
\text { total beta }\end{array}$ & $\begin{array}{l}\text { ND } \\
\text { ND }\end{array}$ \\
\hline $\begin{array}{l}\text { EP-305B-01-S } \\
\text { (305-B Building) } \\
\text { [PNNL] }\end{array}$ & 10.0 & HEPA & $1.5 \mathrm{E}+07$ & 0.5 & $\begin{array}{l}\text { total alpha } \\
\text { total beta }\end{array}$ & $\begin{array}{c}\text { ND } \\
4.4 \text { E-09 }\end{array}$ \\
\hline $\begin{array}{l}\text { EP-306W-03-V } \\
\text { (306-W Building) } \\
\text { [PNNL] }\end{array}$ & 8.8 & HEPA & $4.0 \mathrm{E}+08$ & 12.7 & $\begin{array}{l}\text { total alpha } \\
\text { total beta }\end{array}$ & $\begin{array}{l}5.0 \mathrm{E}-08 \\
5.0 \mathrm{E}-07\end{array}$ \\
\hline $\begin{array}{l}\text { 340-B-BLDG } \\
\text { (340 Complex) } \\
\text { [FDH] }\end{array}$ & 11.6 & HEPA & $9.3 \mathrm{E}+05$ & $<0.1$ & $\begin{array}{l}\text { total alpha } \\
\text { total beta }\end{array}$ & $\begin{array}{l}\text { ND } \\
\text { ND }\end{array}$ \\
\hline $\begin{array}{l}\text { 340-DECON } \\
\text { (340 Complex) } \\
{[\mathrm{FDH}]}\end{array}$ & 3.0 & HEPA & $1.1 \mathrm{E}+08$ & 3.3 & $\begin{array}{l}\text { total alpha } \\
\text { total beta }\end{array}$ & $\begin{array}{c}\text { ND } \\
4.3 \mathrm{E}-07\end{array}$ \\
\hline $\begin{array}{l}\text { EP-318-01-S } \\
\text { (318 Building) } \\
\text { [PNNL] }\end{array}$ & $12: 0$ & None & $1.2 \mathrm{E}+08$ & 3.8 & $\begin{array}{l}\text { total alpha } \\
\text { total beta }\end{array}$ & $\begin{array}{l}7.5 \mathrm{E}-08 \\
1.5 \mathrm{E}-06\end{array}$ \\
\hline $\begin{array}{l}\text { EP-320-01-S } \\
\text { (320 Building) } \\
\text { [PNNL] }\end{array}$ & 12.1 & HEPA & $5.3 \mathrm{E}+08$ & 16.8 & $\begin{array}{l}\text { total alpha } \\
\text { total beta }\end{array}$ & $\begin{array}{l}8.6 \mathrm{E}-08 \\
1.3 \mathrm{E}-06\end{array}$ \\
\hline $\begin{array}{l}\text { EP-320-02-S } \\
\text { (320 Building) } \\
\text { [PNNL] }\end{array}$ & 9.7 & HEPA & $7.6 \mathrm{E}+06$ & 0.2 & $\begin{array}{l}\text { total alpha } \\
\text { total beta }\end{array}$ & $\begin{array}{l}1.8 \mathrm{E}-09 \\
2.8 \mathrm{E}-08\end{array}$ \\
\hline $\begin{array}{l}\text { EP-320-03-S } \\
\text { (320 Building) } \\
\text { [PNNL] }\end{array}$ & 7.9 & HEPA & $6.5 \mathrm{E}+06$ & 0.2 & $\begin{array}{l}\text { total alpha } \\
\text { total beta }\end{array}$ & $\begin{array}{c}\text { ND } \\
7.2 \mathrm{E}-09\end{array}$ \\
\hline $\begin{array}{l}\text { EP-320-04-S } \\
\text { (320 Building) } \\
\text { [PNNL] }\end{array}$ & 7.9 & HEPA & $5.9 \mathrm{E}+06$ & 0.2 & $\begin{array}{l}\text { total alpha } \\
\text { total beta }\end{array}$ & $\begin{array}{c}\mathrm{ND} \\
1.1 \mathrm{E}-08\end{array}$ \\
\hline $\begin{array}{l}\text { EP-323-01-S } \\
\text { (323 Building) } \\
\text { [PNNL] }\end{array}$ & 4.9 & HEPA & $7.0 \mathrm{E}+07$ & 2.2 & $\begin{array}{l}\text { total alpha } \\
\text { total beta }\end{array}$ & $\begin{array}{l}1.6 \mathrm{E}-08 \\
1.3 \mathrm{E}-07\end{array}$ \\
\hline $\begin{array}{l}\text { EP-326-01-S } \\
\text { (326 Building) } \\
\text { [PNNL] }\end{array}$ & 14.5 & HEPA & $8.2 \mathrm{E}+08$ & 26.0 & $\begin{array}{l}\text { total alpha } \\
\text { total beta }\end{array}$ & $\begin{array}{l}5.2 \mathrm{E}-07 \\
7.6 \mathrm{E}-06\end{array}$ \\
\hline
\end{tabular}


Table 2-2

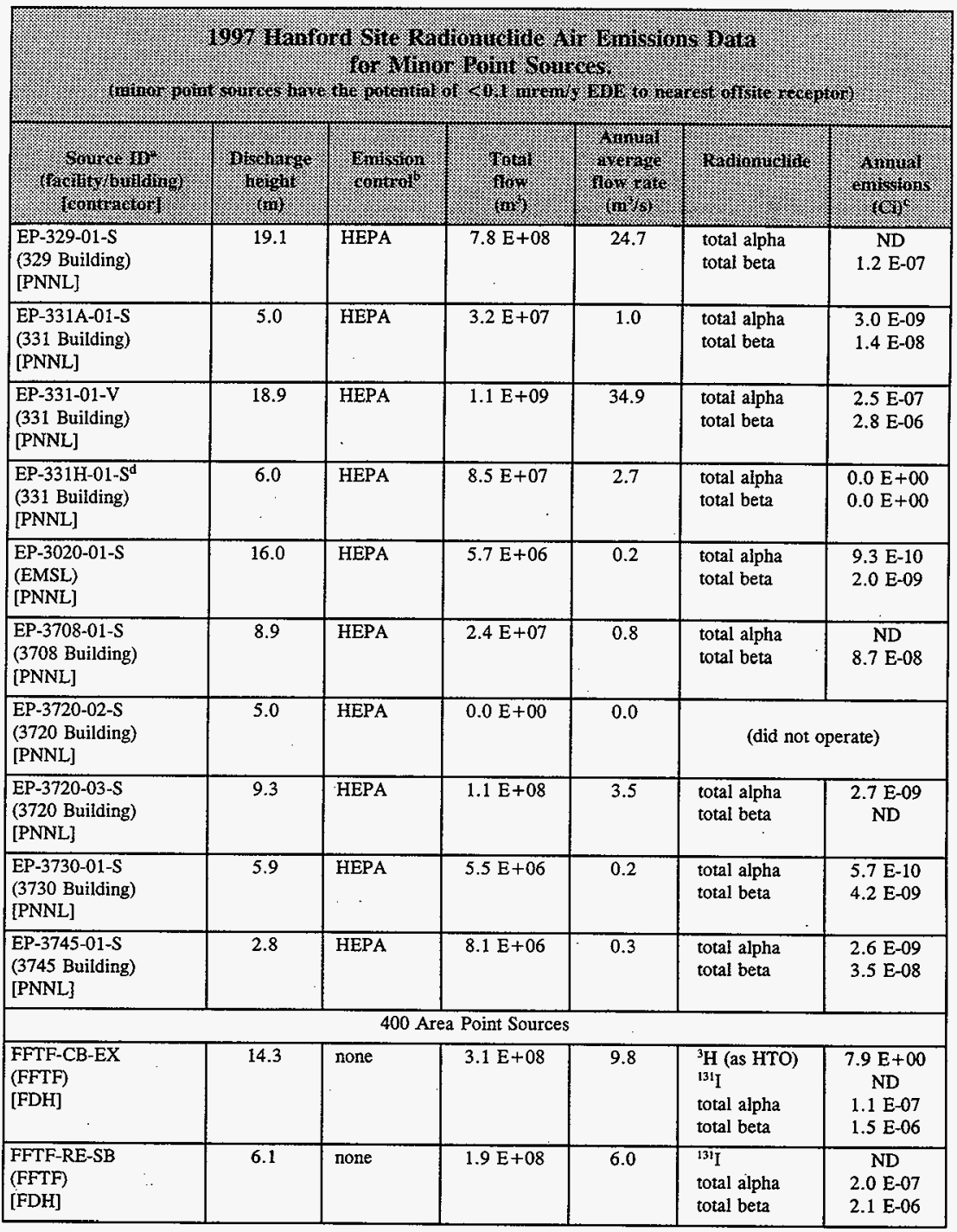




\section{$\varepsilon I-\tau$}

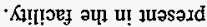

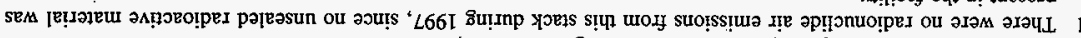

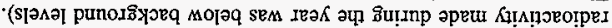

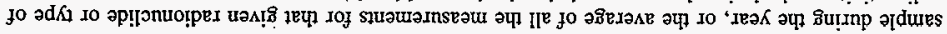

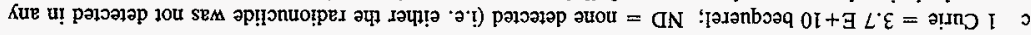
'Ionuos

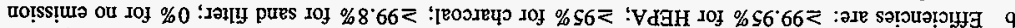

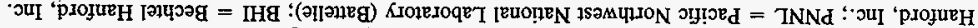

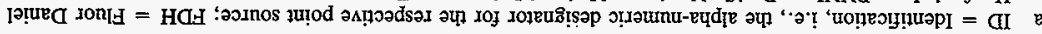
:SशION

\begin{tabular}{|c|c|c|c|c|c|c|}
\hline $\begin{array}{c}80-30.6 \\
\text { aN }\end{array}$ & 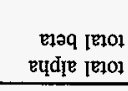 & $\varepsilon \cdot L$ & $80+\exists \varepsilon \tau$ & $\mathrm{Vd} \mathrm{AH}$ & $\underline{L}[I]$ & $\begin{array}{r}\text { [HQS] } \\
(H S W W) \\
\text { [9-[-LED }\end{array}$ \\
\hline $\begin{array}{l}L 0^{\circ}-36{ }^{\circ} S \\
80^{-} \Xi S^{\circ} S \\
\end{array}$ & 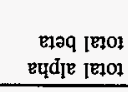 & $\varepsilon L$ & $80+3 \varepsilon \tau$ & $\mathrm{Vd} \exists \mathrm{H}$ & $1 \cdot 6$ & $\begin{array}{r}\text { [HGH] } \\
\text { GSVW) } \\
\text { LSPNW- } L E \text { D } \\
\end{array}$ \\
\hline $\begin{array}{l}\angle 0^{-}-\exists Z{ }^{\prime} Z \\
80^{-} \mathrm{B} L I\end{array}$ & 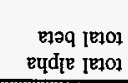 & $s \tau$ & $\angle 0+30.8$ & คưu & 8.8 & 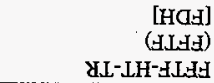 \\
\hline (5) & 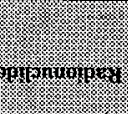 & 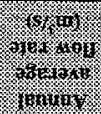 & 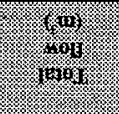 & 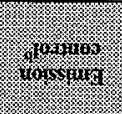 & 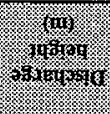 & 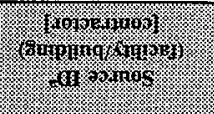 \\
\hline & 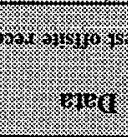 & 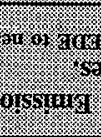 & 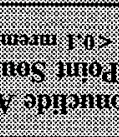 & 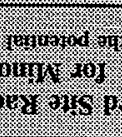 & 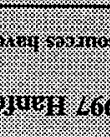 & 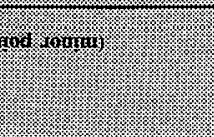 \\
\hline
\end{tabular}

(s1ววบs 8)

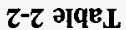


Table 2-3

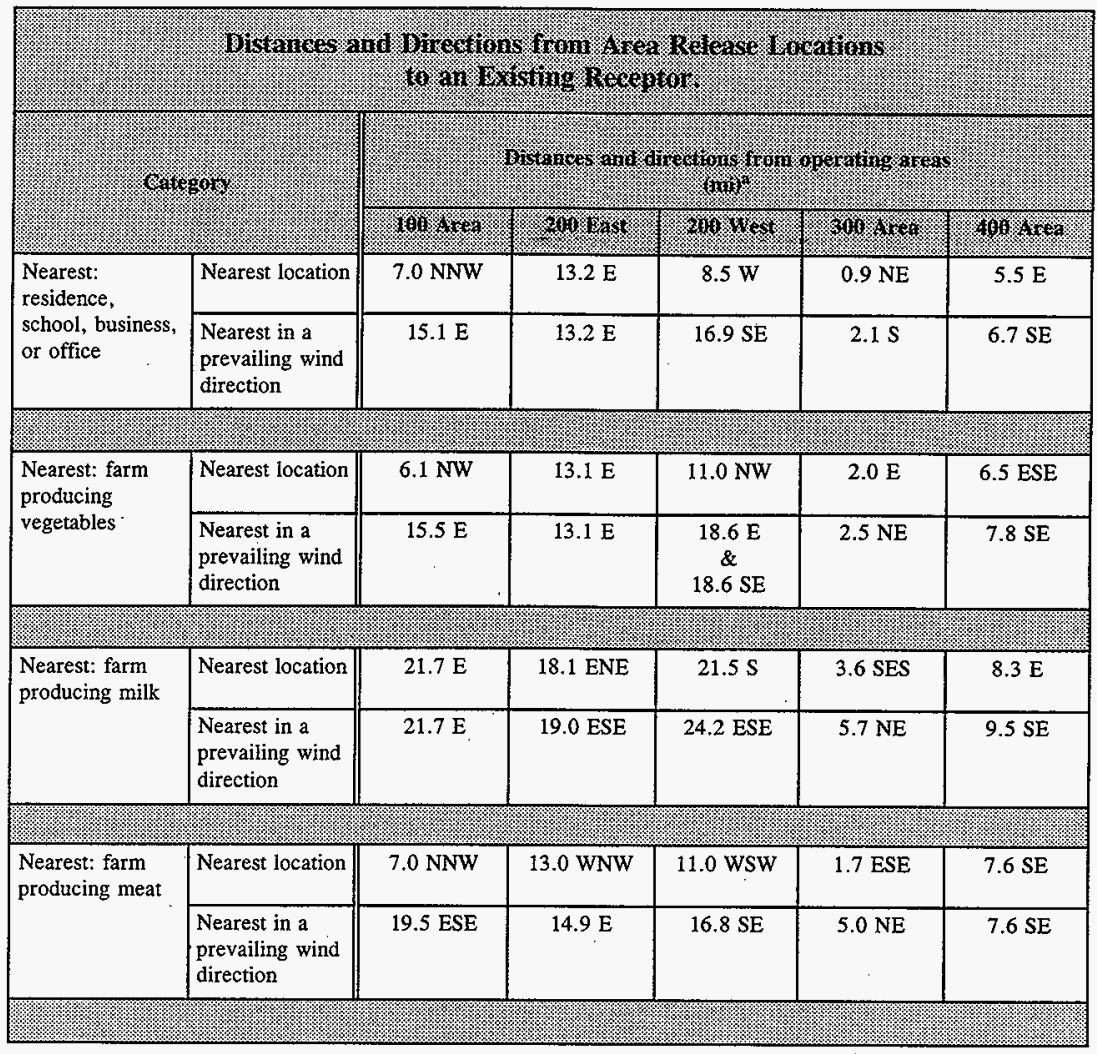

Note:

a $1 \mathrm{mi}=1.609 \mathrm{~km}$. 
DOE/RL-98-33

\subsection{POINT SOURCE EMISSION DOSE ASSESSMENTS}

\subsection{DESCRIPTION OF POINT SOURCE EMISSIONS DOSE MODEL}

The CAP88-PC computer code (EPA 1992) was used to demonstrate compliance with the $10 \mathrm{mrem} / \mathrm{y}$ EDE standard contained in 40 CFR Part 61 Subpart $\mathrm{H}$ and WAC 246-247. Note that the federal and state regulatory agencies use the same dose-standard; however, the constituent doses used to determine compliance with each agency's standard are different. Because the Hanford Site has numerous release points that are widely separated, it was necessary to determine the point at which the maximum dose would be received from combined air emissions at all locations. Emission points used for modeling purposes were centered on each of the major operating areas that released radionuclides to the atmosphere during 1997. The reference point for the 100 Areas was the spent fuel storage basin at the 105-KE Building. In the case of the 200 Areas, the reference points were the major sources in the 200 East and 200 West Areas, the PUREX Plant and PFP, respectively. Reference locations for the 300 Area were the 324 Building, for non-radon emissions, and the 327 Building, for ${ }^{220} \mathrm{Rn}$ and ${ }^{222} \mathrm{Rn}$ emissions. FFTF was used as the 400 Area reference location.

Point source emissions from the major operating areas are listed in Table 3-1. The location of the MEI selected for the compliance analysis was a farm at Sagemoor Road, which is directly across the Columbia River from the Hanford Site boundary to the east of the 300 Area. Table 3-2 lists the effective dose equivalent to this receptor from 1997 releases of individual radionuclides at each location, and the location of the emission sources relative to the Sagemoor Road receptor. The receptor at Ringold received an EDE comparable to the receptor at Sagemoor Road from air emissions, excluding ${ }^{220} \mathrm{Rn}$ and ${ }^{222} \mathrm{Rn}$ emissions. When ${ }^{220} \mathrm{Rn}$ and ${ }^{222} \mathrm{Rn}$ emissions are included, the EDE to the receptor at Sagemoor Road is higher than at Ringold.

\subsection{SUMMARY OF INPUT PARAMETERS}

Dose calculations were performed using established standard parameters for the Hanford Site and its environment (PNL 1993). Release estimates, grouped by major operating area, were used to perform dose calculations (Table 3-1). Releases from point sources were modeled using an effective discharge height of $10 \mathrm{~m}(33 \mathrm{ft})$ for all release locations other than the 200 Area facilities, which were assumed to have an effective release height of $89 \mathrm{~m}(292 \mathrm{ft})$. Releases reported as total alpha or total beta were generally evaluated as ${ }^{239} \mathrm{Pu}$ or ${ }^{90} \mathrm{Sr}$, respectively, to provide a conservative estimate of the expected impact. In the case of 400 Area, some releases reported as total beta measurements were modeled as ${ }^{137} \mathrm{Cs}$, based on facility specific information. 
Radionuclide data used for the dose calculations are listed in Appendix A; all other radionuclide-specific parameters were the default values provided by CAP88-PC data libraries. The maximum individual exposure and consumption parameters were those determined previously for the Hanford Site, and are also included in Appendix A.

Parameters used for the ingestion pathway assumed that the receptor's entire diet was produced at the residence location, the "local" food production option in CAP88-PC.

Radionuclide air concentrations at the receptor location were determined using site-specific meteorological data for each release point. Joint frequency distributions and CAP88-PC wind files were prepared from data collected at weather stations in each of the operating areas, and these represent the average of hourly data taken during 1997. This information was used to determine 1997 annual average dispersion coefficients for each of the major release points (Appendix A).

\subsection{COMPLIANCE ASSESSMENT}

\subsubsection{Regulatory Limit of 40 CFR 61, Subpart $H$}

During 1997, the combined dose to a receptor at the Sagemoor farm attributed to the air emissions from all of the point sources, excluding ${ }^{220} \mathrm{Rn}$ and ${ }^{222} \mathrm{Rn}$ emissions, was $1.2 \mathrm{E}-03 \mathrm{mrem}(1.2 \mathrm{E}-05 \mathrm{mSv}) \mathrm{EDE}$. This dose is less than $0.02 \%$ of the $10 \mathrm{mrem} / \mathrm{y}$ standard, specified in 40 CFR 61 Subpart $\mathrm{H}$.

As shown in Table 3-2, this dose resulted mainly from emissions related to activities at facilities in the 200 and 300 Areas. Activities in the 300 Area accounted for approximately $35 \%$ of the offsite dose, and the contributions from 200 East and 200 West Areas accounted for $32 \%$ and $15 \%$, respectively, of the offsite dose. The emissions from the 400 Area accounted for $17 \%$ of the offsite dose. The emissions from the 100 Area contributed less than $1 \%$ of the total. Radionuclides, other than radon and thoron, contributing more than $10 \%$ of the total dose included ${ }^{3} \mathrm{H}(44 \%)$, ${ }^{129} \mathrm{I}(25 \%)$, and ${ }^{239 / 240} \mathrm{Pu}(20 \%)$.

The MEI for the Hanford Site compliance calculations is located approximately $1.5 \mathrm{~km}$ $(0.93 \mathrm{mi})$ east of the 324 Building, in the 300 Area. The 324 Building was chosen to represent the source of all 300 Area non-radon emissions, because it is the major emissions source in the 300 Area closest to the MEI.

Again for emphasis:

40 CFR 61 SUBPART H

The Hanford Site's EDE for 1997 is 1.2 E-03 mrem (1.2 E-05 mSv) 
This dose is slightly lower than the annual dose for the previous years. The 1990 through 1992 doses were modeled using the mainframe version of CAP88 and an MEI location at Ringold. The 1993 through 1997 doses were modeled using CAP88-PC and an MEI location at Sagemoor Road. The personal computer version of CAP88, CAP88-PC, relies on default parameters more than the mainframe version. Figure 3-1 displays the offsite MEI doses, per 40 CFR 61 Subpart $H$ requirements, attributable to Hanford Site emissions since 1990 , the first year this report was issued.

Figure 3-1. Historical 40 CFR 61, Subpart H Doses to the MEI.

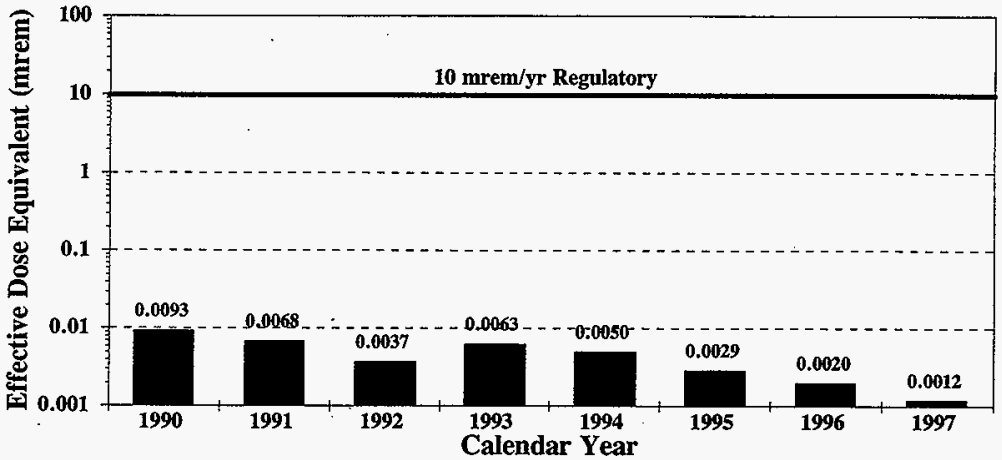

\subsubsection{Regulatory Limit of WAC 246-247}

During 1997, the combined dose to a receptor at the Sagemoor farm attributed to the air emissions from all of Hanford's radioactive emissions sources, including ${ }^{220} \mathrm{Rn},{ }^{222} \mathrm{Rn}$ and emissions from diffuse and fugitive sources, was $2.6 \mathrm{E}-02 \mathrm{mrem}(2.6 \mathrm{E}-04 \mathrm{mSv}) \mathrm{EDE}$. This dose is less than $0.3 \%$ of the $10 \mathrm{mrem} / \mathrm{y}$ standard, specified in the WAC 246-247.

Significant radionuclides, when all emission sources are considered, contributing more than $10 \%$ of the compliance dose included ${ }^{234} \mathrm{U}(35 \%)$ and ${ }^{238} \mathrm{U}(36 \%)$. During 1997 , all of the uranium emissions were attributed to diffuse and fugitive sources.

The MEI for the Hanford Site compliance calculations is located approximately $1.5 \mathrm{~km}$ $(0.93 \mathrm{mi})$ east of the 324 Building, in the 300 Area.

Again for emphasis:

WAC 246-247

The Hanford Site's EDE for 1997 is $2.6 \mathrm{E}-02 \mathrm{mrem}$ (2.6 E-04 mSv) 
Table 3-1

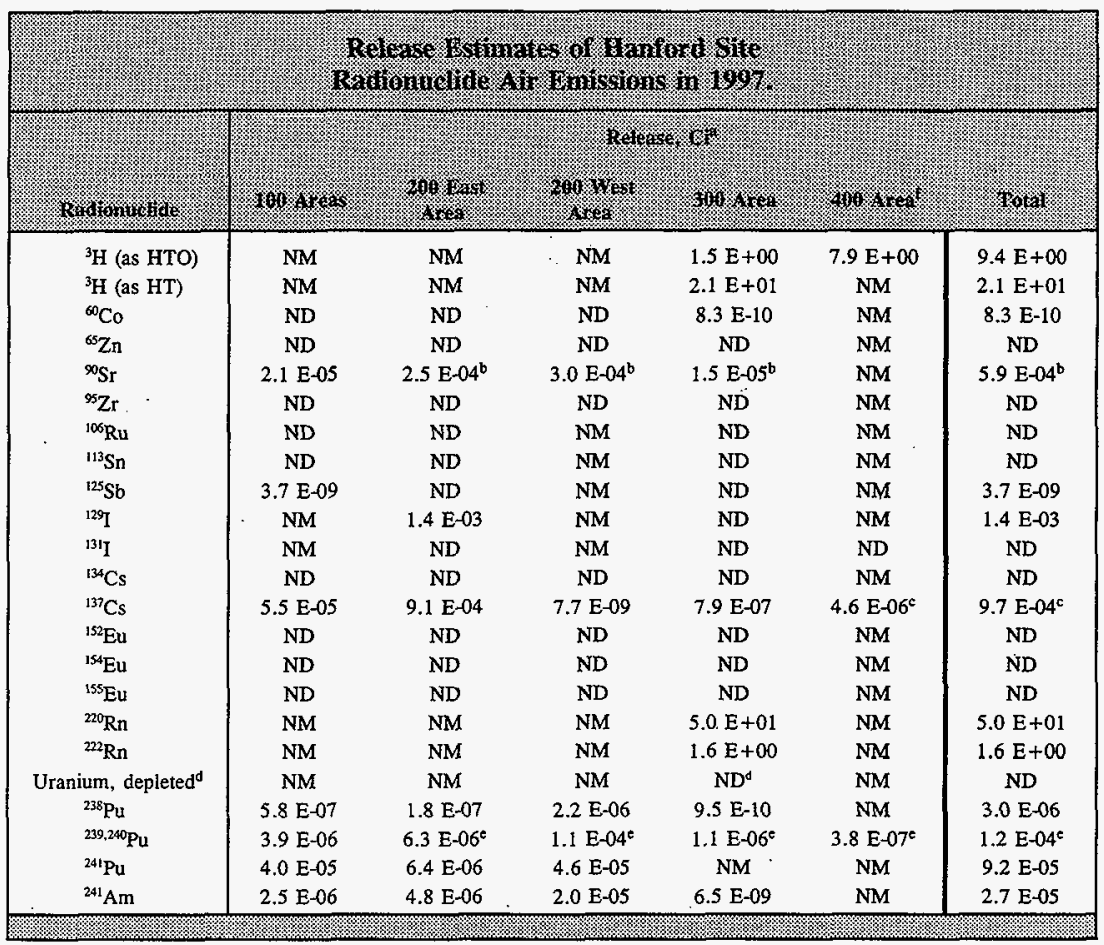

Notes:

a $1 \mathrm{Ci}=3.7 \mathrm{E}+10 \mathrm{Becquerel}$; ND $=$ not detected (i.e. either the radionuclide was not detected in any sample during the year, or the average of all the measurements for that given radionuclide or type of radioactivity made during the year was below background levels); $\mathrm{NM}=$ not measured.

b This value includes total beta release data. Total beta and unspecified beta results assumed to be ${ }^{90} \mathrm{Sr}$ for dose calculations.

c This value includes total beta release data. Total beta results assumed to be ${ }^{137} \mathrm{Cs}$ for dose caiculations from FFTF emissions.

d Determined from totai alpha measurements. Assumed to be depleted uranium consisting of $63.478 \mathrm{Ci} \%{ }^{238} \mathrm{U}, 0.821$ $\mathrm{Ci} \%{ }^{235} \mathrm{U}$, and $35.701 \mathrm{Ci} \%{ }^{234} \mathrm{U}\left(99.797 \mathrm{Wt} \%{ }^{238} \mathrm{U}, 0.200 \mathrm{Wt} \%{ }^{235} \mathrm{U}\right.$, and $\left.0.003 \mathrm{Wt} \%{ }^{234} \mathrm{U}\right)$.

e This value includes total alpha release data. Total alpha and unspeciffed aipha results assumed to be ${ }^{239240} \mathrm{Pu}$ for dose calculations. 
Table 3-2

\begin{tabular}{|c|c|c|c|c|c|c|c|}
\hline & 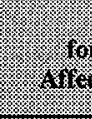 & 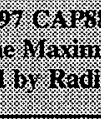 & 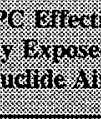 & 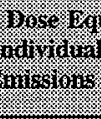 & 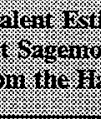 & 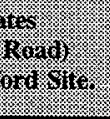 & \\
\hline & & 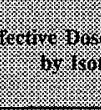 & (1) & 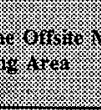 & & ris & 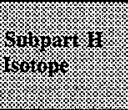 \\
\hline Gningristix & 100 & (20\%) & 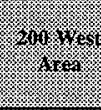 & 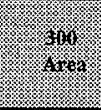 & 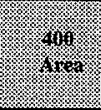 & (x) & 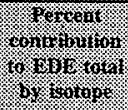 \\
\hline Hecentor & 4. & (2) & . & $1 \%$ & (1) & (m) & \\
\hline $\begin{array}{l}{ }^{3} \mathrm{H} \\
{ }^{60} \mathrm{CO}\end{array}$ & & & & $\begin{array}{r}3.1 \mathrm{E}-04^{\mathrm{b}} \\
1.8 \mathrm{E}-09\end{array}$ & $2.0 \mathrm{E}-04$ & $\begin{array}{l}5.1 \mathrm{E}-04 \\
1.8 \mathrm{E}-09\end{array}$ & $\begin{array}{l}44 \% \\
<1 \%\end{array}$ \\
\hline${ }^{90} \mathrm{Sr}^{\mathrm{c}}$ & 3.2 E-07 & 6.2 E-06 & 5.8 E-06 & $1.5 \mathrm{E}-05$ & & $2.7 \mathrm{E}-05$ & $2 \%$ \\
\hline${ }^{125} \mathrm{Sb}$ & 1.3 E-11 & & & & & $1.3 \mathrm{E}-11$ & $<1 \%$ \\
\hline${ }^{129} \mathrm{~T}$ & & $2.9 \mathrm{E}-04$ & & & & $2.9 \mathrm{E}-04$ & $25 \%$ \\
\hline${ }^{137} \mathrm{Cs}$ & $1.8 \mathrm{E}-06$ & $4.8 \mathrm{E}-05$ & $3.2 \mathrm{E}-10$ & $1.6 \mathrm{E}-06$ & $1.2 \mathrm{E}-06^{\mathrm{d}}$ & $5.3 \mathrm{E}-05$ & $5 \%$ \\
\hline${ }^{238} \mathrm{Pu}$ & $5.6 \mathrm{E}-07$ & $2.5 \mathrm{E}-07$ & 2.4 E-06 & $6.4 \mathrm{E}-08$ & & $3.3 \mathrm{E}-06$ & $<1 \%$ \\
\hline${ }^{239 / 240} \mathrm{Pu}^{e}$ & 4.0 E-06 & 9.5 E-06 & $1.3 \mathrm{E}-04$ & 8.1 E-05 & 3.4 E-06 & 2.3 E- -04 & $20 \%$ \\
\hline${ }^{24 !} \mathrm{Pu}$ & $6.5 \mathrm{E}-07$ & $1.5 \mathrm{E}-07$ & $8.5 \mathrm{E}-07$ & & & $1.7 \mathrm{E}-06$ & $<1 \%$ \\
\hline${ }^{241} \mathrm{Am}$ & $4.0 \mathrm{E}-06$ & 1.1 E-05 & $3.6 \mathrm{E}-05$ & $7.3 \mathrm{E}-07$ & & $5.2 \mathrm{E}-05$ & $4 \%$ \\
\hline 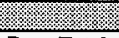 & Y & S & (3) & 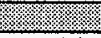 & & 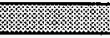 & 3 \\
\hline Dose Totals & $1.1 \mathrm{E}-05$ & $3.7 \mathrm{E}-04$ & $1.7 \mathrm{E}-04$ & $4.1 \mathrm{E}-04$ & 2.0 E-04 & $1.2 \mathrm{E}-03$ & $100 \%$ \\
\hline
\end{tabular}

Notes:

a $1 \mathrm{mrem}=1 \mathrm{E}-02 \mathrm{mSv} ; 1 \mathrm{~km}=6.21 \mathrm{E}-01 \mathrm{mi}$.

b The HT refease was converted to equivalent curies of HTO for dose modeling purposes because the CAP-88 models assume that tritium is in the oxidized form. For this assessment, $1 \mathrm{Ci}$ of $\mathrm{HT}$ was assumed to be equivalent to $0.01 \mathrm{Ci}$ of HTO (Brown et al. 1990).

c This value includes total beta release data. Total beta and unspecified beta results were assumed to be ${ }^{x} \mathrm{Sr}$ for dose calculations at all facilities other than FFTF.

d This value includes total beta release data. Total beta results were assumed to be ${ }^{137} \mathrm{Cs}$ for dose calculations from FFTF emissions.

e This value includes total alpha release data. Total alpha and unspecified alpha results were assumed to be ${ }^{239240} \mathrm{Pu}$ for dose calculations. 


\subsection{METEOROLOGICAL DATA}

Radionuclide air emissions disperse once the emissions enter the atmosphere. Atmospheric dispersion models predict the degree of dilution and the magnitude of resulting air concentrations at downwind locations. Site-specific measurements of the occurrence frequencies for wind speed, wind direction, and atmospheric stability are used in the models. The dispersion models yield annual average dispersion factors (in units of $s / \mathrm{m}^{3}$ ). Combining these factors with annual average release rates.predicts average radionuclide air concentrations for the year. Annual average dispersion factors around the 100, 200, 300, and 400 Areas for 1997 are in Appendix A.

\subsection{UNPLANNED RELEASES OF RADIONUCLIDES TO THE ATMOSPHERE}

During 1997, two unplanned releases of radioactive material to the atmosphere occurred one at the Waste Encapsulation and Storage Facility (WESF) and one at the 324 Building.

On January 1st, the WESF 296-B-10 stack had elevated emissions for a period of approximately 90 minutes. The elevated emissions were due to stormwater flooding in the K-3 filter pit, which allowed radioactive liquid into the ducting downstream of the online HEPA filter bank. Once the problem was discovered, the emissions were rerouted through the alternate HEPA filter bank. This event was limited to 90 minutes because the 296-B-10 stack is equipped with effluent monitoring and mitigation systems that exceeded the minimum regulatory requirements. It was estimated, using effluent sample results, that $1.4 \mathrm{E}-04 \mathrm{Ci}$ of ${ }^{90} \mathrm{Sr}$ and $1.4 \mathrm{E}-05 \mathrm{Ci}$ of ${ }^{137} \mathrm{Cs}-137$ were released within the 90 minute period.

On June 13th, the 324 Building EP-324-01-S stack had an inadvertent release of several radon isotopes. A nearly instantaneous release occurred when a seal was broken during the repackaging of several medical isotopes, ${ }^{226} \mathrm{Ra},{ }^{227} \mathrm{Ac}$, and ${ }^{228} \mathrm{Th}$, created for cancer therapy. These medical isotopes produce radon gas by radioactive decay. Sampling and monitoring for the radon isotopes was not required and was not performed during this activity. A worst-case estimate of the release was calculated using the source term data and the facility operating data. The release was conservatively estimated to be no more than 3.1 $\mathrm{Ci}$ of ${ }^{219} \mathrm{Rn}, 46 \mathrm{Ci}$ of ${ }^{220} \mathrm{Rn}$, and $0.50 \mathrm{Ci}$ of ${ }^{222} \mathrm{Rn}$. The dose to members of the public was minimized because of the wind direction during the event, which happened to be opposite of the prevailing wind direction. The wind was coming out of the east at the time of event, which dispersed the short-lived contaminants out over the Hanford Site.

On May 14th, an explosion occurred in the Plutonium Reclamation Facility (PRF) at the Plutonium Finishing Plant (PFP), blowing open several inside doorways and blowing a hole in the roof of the facility. The explosion did not cause any radioactive material to be released to the environment, even though radioactive material exists in this facility. This facility is ventilated by the $291-Z-1$ stack. Effluent sampling data and near-facility ambient 
air monitoring station data indicated no increased release of radioactive material occurred as a result of the explosion. Contamination smears of the opening in the roof also confirmed that no radioactive material was released through the opening.

No other unplanned releases to the atmosphere occurred from facilities at the Hanford Site, during 1997. Releases from actively ventilated point sources, with a potential to release radioactivity, on the Hanford Site were routinely sampled. The resulting quantities of radionuclides released and the corresponding EDEs are reported in Sections 2.0 and 3.0 of this document.

\subsection{ADDITIONAL INFORMATION}

\subsubsection{Construction Projects and Modifications Exempted from 40 CFR 61.96}

No waivers or exemptions of the approval process under 40 CFR 61.96 were exercised in 1997. In late 1992, the Environmental Protection Agency (EPA) determined that some of the emission units on the Hanford Site were not in compliance with the requirements specified in 40 CFR 61, Subpart $H$ (NESHAP regulations). A Federal Facilities Compliance Agreement (FFCA) for NESHAP regulation was established between DOE-RL and EPA Region 10, as a result of the noncompliant emission units. Consequently, EPA approval is required for all construction or modification projects with the potential to increase radionuclide air emissions, until completion of all milestones listed in the FFCA.

\subsubsection{Doses to the MEI due to Radon and Thoron Emissions}

The dose to the $\mathrm{MEI}$ due to ${ }^{220} \mathrm{Rn}$ (thoron) and ${ }^{222} \mathrm{Rn}$ (radon) emissions are included in Table 3-3. These emissions result in a significant portion of the dose received by the MEI, but do not require monitoring per state and federal regulations. Emissions of radon isotopes are specifically excluded from 40 CFR Part 61, Subpart H regulations. The WAC 246-247 only exclude radon emissions from naturally occurring sources and does not exclude radon emissions from technologically enhanced sources. Regulations only require that radionuclides which could contribute greater than $10 \%$ of the potential effective dose equivalent for a release point to be measured. Radon and thoron emissions are well below this threshold. However, it is currently Hanford's policy to include all radionuclide emissions that were measured in this report. As a result, doses associated with these emissions are reported separately from those in Table 3-3. Sections 5.2, 5.3, and 5.4 contain additional information on radon and thoron emissions. 
Table 3-3

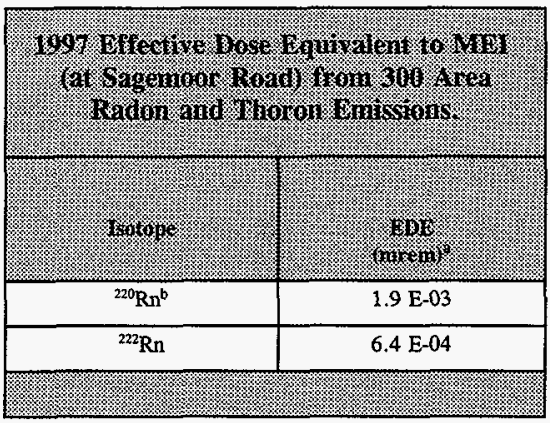

Notes:

a $1 \mathrm{mrem}=1 \mathrm{E}-02 \mathrm{mSv}$.

b Releases of ${ }^{220} \mathrm{Rn}$ were modeled by assuming that the short-lived gas had decayed to the next long-lived member of the decay chain, ${ }^{212} \mathrm{~Pb}$, prior to transport offsite.

\subsubsection{Supplementary Data from Dose Modeling Results}

Unit dose factors, organized by operating area and radionuclide, were calculated from the releases and dose modeling results. These factors were then used to calculate the point source and radionuclide specific doses included in Table 2-1. Table 3-4 contains the EDE to the MEI and percentage of the sitewide dose, displayed by major emission unit. 


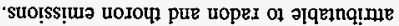

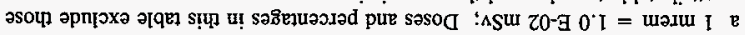
$: 210 \mathrm{~N}$

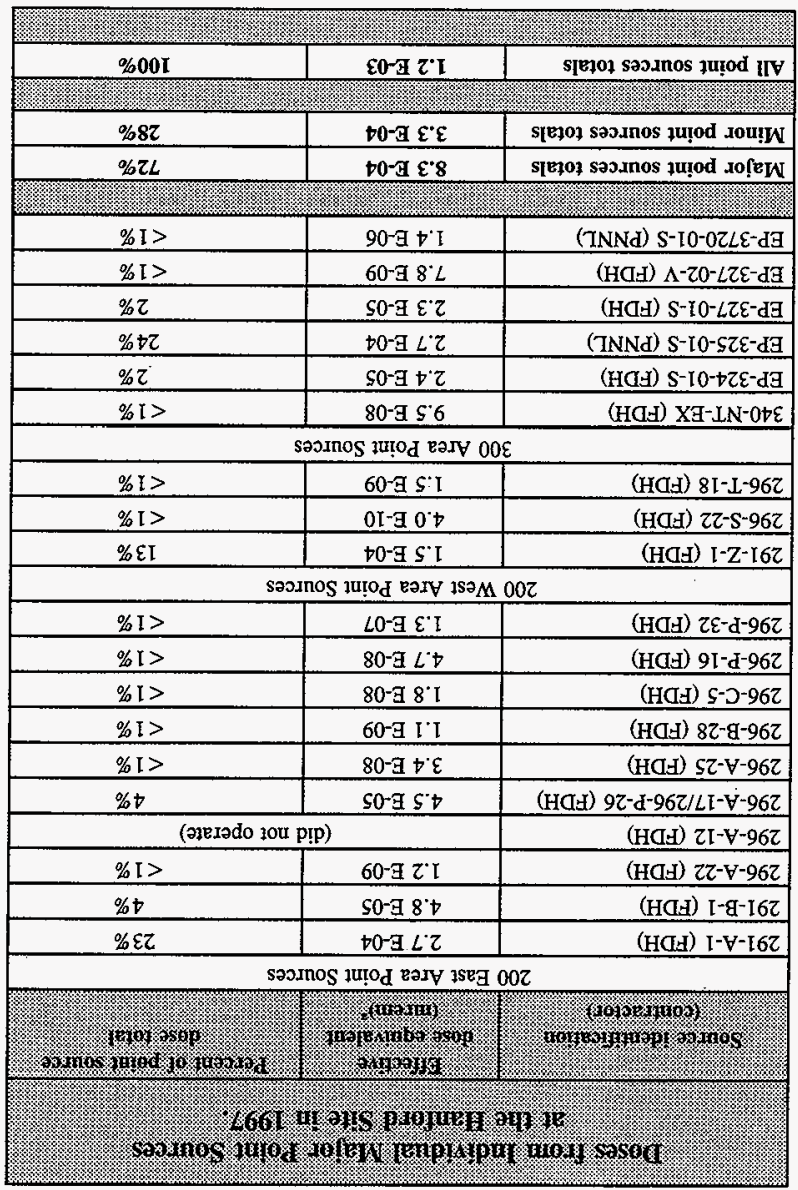

t-E ə]qeI 


\subsubsection{Revised CY-1996 Release from 296-T-7 Stack}

The CY-1996 releases reported last year in the Radionuclide Air Emissions Report for the Hanford Site Calendar Year 1996 (DOE-RL 1997a) were slightly under reported. The revised values were no more than $8.3 \%$ greater than the values in the CY-1996 report. The revised releases did not change any of the dose modeling results, since the releases from the 296-T-7 stack are relatively insignificant when compared to other emissions sources at Hanford.

An error in the calculations was discovered while performing the calculations for the CY-1997 report. Calculations were performed indicating the 296-T-7 stack did not operate during two periods of time (5/02/96 to $5 / 07 / 96$ and $10 / 31 / 96$ to $12 / 31 / 96)$. In 1998 , it was determined that the 296-T-7 stack had actually operated intermittently during those two time periods, causing the original releases to be slightly under reported.

Table 3-5

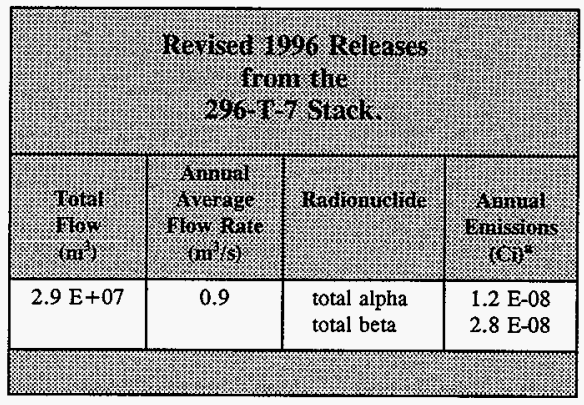

Note:

a 1 Curie $=3.7 \mathrm{E}+10$ becquerel. 


\subsection{CERTIFICATION}

This certification applies only to Sections $1.0,2.0$, and 3.0 .

"I certify under penalty of law that I have personally examined and am familiar with the information submitted herein and, based on my inquiry of those individuals immediately responsible for obtaining the information, I believe that the submitted information is true, accurate, and complete. I am aware that there are significant penalties for submitting false information, including the possibility of fine and imprisonment. See, 18 U.S.C. 1001."
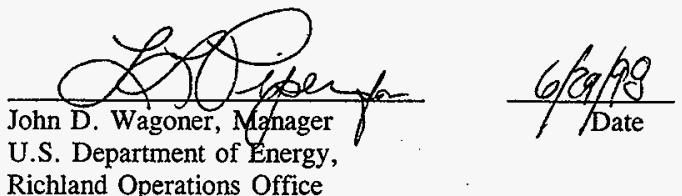

Richland Operations Office 


\section{DOE/RL-98-33}

This page intentionally left blank. 


\subsection{DIFFUSE AND FUGITIVE SOURCES OF EMISSIONS}

Diffuse and fugitive sources include all radioactive emission sources that are not actively ventilated or are not routinely sampled (e.g. passively ventilated tank vents, outdoor surface contamination areas, cracks between cover blocks, etc...). All diffuse and fugitive emission sources are monitored by the Hanford Site's diffuse and fugitive emissions monitoring program, as described in Section 4.1. There are numerous types of these sources in and around the facilities, as described in Section 4.3.

WAC 246-247, requires facilities to monitor nonpoint (diffuse) and fugitive emissions of radioactive material. DOE Headquarters has requested that estimated doses to the public, related to radioactive emissions from both point sources along with diffuse and fugitive sources, be included in this report. This request is based on the requirement in 40 CFR 61 , Subpart $H$, that the dose from all potential emission sources at DOE facilities not exceed $10 \mathrm{mrem} / \mathrm{y}$ EDE to any member of the public. This section describes the diffuse and fugitive emissions monitoring program and estimates the EDE to the public, attributable to diffuse and fugitive sources.

Currently, all nuclear material production facilities on the Hanford Site are shutdown or are in standby mode. Only waste minimization and stabilization processes continue to operate. In the past, when the Hanford Site was operating at or near full capacity, point source emissions were easily detected. Now, however, emissions from point sources have diminished in most instances to background levels. Therefore, the environmental contribution from diffuse and fugitive emissions has become a larger percentage of the total radioactivity attributable to activities on the Hanford Site, even though diffuse and fugitive emissions have remained relatively constant.

The standard approach for assessing offsite doses from forced ventilation exhaust points is by applying atmospheric transport models to measured releases. The assessment of offsite doses from diffuse and fugitive emissions is not as straightforward. The assessment of offsite doses from diffuse and fugitive emissions is complicated by the difficulties involved in quantifying the emissions from the source term. Methods for quantifying diffuse and fugitive emissions are still being developed because of complicating factors such as:

(1) difficulty in accurately quantifying air flow from the source, (2) more complex influences from meteorological conditions, and (3) exceedingly low detection levels. Passively ventilated emissions have been considered diffuse in nature because the emissions lack a measurable flow.

Passively ventilated point sources, breather vents and other openings on tanks, vaults, and other structures are potential conduits of radioactive emissions. Airborne radionuclides inside vented structures can be released via passive air exchanges, typically attributed to ambient temperature and pressure changes. However, it is difficult to accurately assess radionuclide releases that might occur under such conditions, particularly when the vent opening is irregularly shaped, or when multiple openings are in close proximity. Passively ventilated point sources are not routinely sampled, where as actively ventilated stacks and 
vents are, since emissions from passively ventilated sources cannot be readily quantified. Therefore, estimates of quantities of radionuclides discharged via diffuse and fugitive emissions sources are made based on data obtained using a comprehensive network of ambient air samplers. Dose and release estimates from diffuse and fugitive sources of radionuclide air emissions are calculated using sample data from these ambient air samplers. Section 4.2 contains the dose and release estimates for the Hanford Site's diffuse and fugitive emissions.

For this report, doses have been calculated for emissions from both actively ventilated point sources and diffuse and fugitive sources. The methods used for calculating diffuse and fugitive emissions are still being refined. Dose calculations for each type of emission are presented separately. As methods for estimating the dose from diffuse and fugitive emissions are refined, more precise amounts representing radionuclides occurring naturally, from nuclear-testing fallout and non-DOE nuclear facilities (Figure 4-1) might be subtracted from ambient air concentrations. Figure 4-1 uses a historical window of sample data to demonstrate several things, the first being how ambient air concentrations from sample locations at the Hanford Site's perimeter differ little from the concentrations measured at distant locations. The figure demonstrates the impact emissions from non-Hanford Site sources can have on diffuse and fugitive emissions estimates. The figure demonstrates the sensitivity of the Hanford Site's Environmental Surveillance Program, such that evidence of global nuclear events can be observed in the sample data.

Figure 4-1. Monthly Average Gross Beta Radioactivity in Airborne Particulate Samples, 1979 through 1989 (PNL 1990).

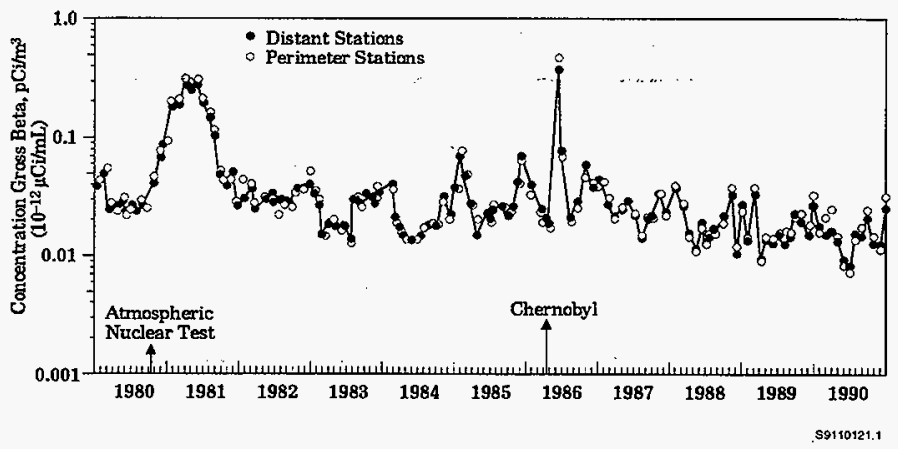




\subsection{DIFFUSE AND FUGITIVE EMISSIONS MONITORING}

On the Hanford Site, two programs monitor diffuse and fugitive emissions, the NearFacility Environmental Monitoring Program and the Environmental Surveillance Program. These two programs monitor locations on and off of the Hanford Site. The intent of these programs is to detect and quantify radiological and nonradiological contaminants and assess their environmental and human health significance. These programs monitor contaminants released to the environment, which might reach humans via all pathways. This report presents monitoring information relative to radiological contaminants for pathways involving atmospheric transport.

\subsubsection{Near-Facility Environmental Monitoring}

Near-facility environmental monitoring is defined as monitoring near facilities that have potentially dispersible radioactivity. Monitoring locations are associated mostly with major nuclear facilities and waste storage or disposal facilities such as burial grounds, tank farms, ponds, cribs, trenches, and ditches.

Routine monitoring activities include sampling and monitoring ambient air, surface contamination, surface-water, external radiation doses, vadose zone, soil, sediment, vegetation, and animals. Samples are collected from known or expected effluent transport pathways, which are generally downwind of potential or actual airborne releases and downgradient of liquid discharges. Atmospheric transport is the most efficient offsite transport vector and thus ambient air is the principle media monitored in the near-facility region, although samples of surface soils, surface water, vadose zone, sediments, vegetation, and animals might also be useful as secondary indicators.

Airborne radioactivity is sampled by a network of continuously operating samplers at 63 locations near facilities: 4 in the 100-N Area, 4 in the 100-K Area, 4 in the 100-D/DR Area, 3 in the $100-\mathrm{B} / \mathrm{C}$ Area, 38 in the 200 Areas, 3 at the Environmental Restoration Disposal Facility (ERDF), 3 at the 300-FF-1 Project (north of the 300 Area), 2 at the 3732 Building in the 300 Area, 1 near the 300 Area Treated Effluent Disposal Facility, and 1 station at the Wye Barricade. The station at the Wye Barricade is collocated with samplers operated by the PNNL Surface Environmental Surveillance Project and the Washington State Department of Health (WDOH). Additional samplers are also used to support specific environmental remediation tasks. Ambient air samplers are primarily located at or near sites

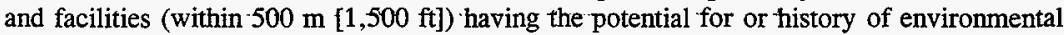
releases. Particulate air samples are analyzed for total alpha activity, total beta activity, gamma emitting isotopes, ${ }^{90} \mathrm{Sr}$, uranium isotopes $\left({ }^{234} \mathrm{U} ;{ }^{235} \mathrm{U}\right.$, and $\left.{ }^{238} \mathrm{U}\right)$, plutonium isotopes ${ }^{238} \mathrm{Pu}$ and ${ }^{239 / 240} \mathrm{Pu}$ ). Gamma emitting isotopes routinely reported include ${ }^{60} \mathrm{Co},{ }^{65} \mathrm{Zn},{ }^{95} \mathrm{Zr}$, ${ }^{106} \mathrm{Ru},{ }^{125} \mathrm{Sb},{ }^{134} \mathrm{Cs},{ }^{137} \mathrm{Cs},{ }^{154} \mathrm{Eu},{ }^{155} \mathrm{Eu}$, and any other detected isotopes. The $100-\mathrm{K}$ Area air samples are also analyzed for ${ }^{241} \mathrm{Am}$ and ${ }^{241} \mathrm{Pu}$. Figure $4-2$ depicts the locations of the ambient air samplers used for near-facility monitoring. 
Figure 4-2. Near-Facility Ambient Air Sampling Locations.

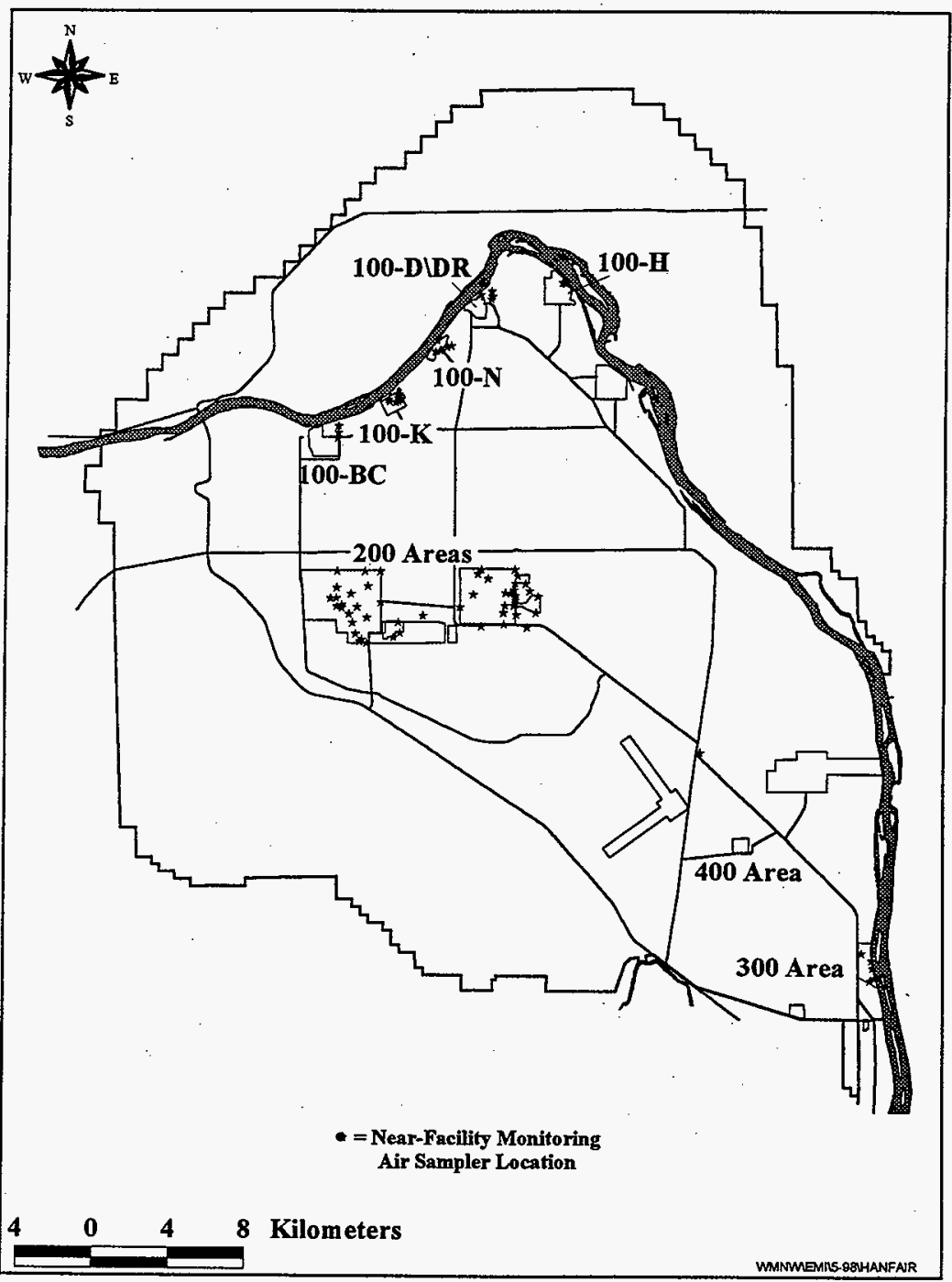


More detailed descriptions of this program can be found in the Hanford Site NearFacility Environmental Monitoring Report (WMNW 1998) and the Hanford Site Environmental Report (PNNL 1998a).

\subsubsection{Environmental Surveillance}

Environmental surveillance encompasses sampling and analyzing for potential radiological contaminants on and off the Hanford Site. Monitoring locations are divided into three surveillance zones located on and off of the Hanford Site.

The first surveillance zone extends from the near-facility monitoring locations to the Hanford Site perimeter. The second surveillance zone consists of a series of perimeter sampling stations near or just inside the Hanford Site boundary and along State Highway 240 . The third surveillance zone consists of nearby and distant sampling locations within an $80 \mathrm{~km}(50 \mathrm{mi})$ radius of the Hanford Site. Background sampling is performed at distant locations unaffected by Hanford Site operations.

Routine surveillance activities include the sampling and monitoring of air, surfacewater, groundwater, food and farm products, fish and wildlife, soil and vegetation, and external radiation. Like the near-facility monitoring program, ambient air sampling is the primary method used in monitoring diffuse and fugitive emissions.

The air surveillance network consists of 39 sampling stations, of which 20 are on the Hanford Site (zone 1), 9 at the Hanford Site's perimeter (zone 2), 8 in nearby communities (zone 3 ), and 2 in distant communities (background locations). This program routinely monitors for radioactive vapors, gases, and aerosols. Vapor, gas, and liquid aerosol sampling and analysis is performed for ${ }^{3} \mathrm{H}$ and ${ }^{129} \mathrm{I}$, at selected locations. Solid aerosols (particulate) are sampled and analyzed for at all sampling locations. Particulate air samples are routinely analyzed for total alpha activity, total beta activity, gamma emitting isotopes, ${ }^{90} \mathrm{Sr}$, uranium isotopes ${ }^{234} \mathrm{U},{ }^{235} \mathrm{U}$, and ${ }^{238} \mathrm{U}$, and plutonium isotopes $\left({ }^{238} \mathrm{Pu}\right.$ and ${ }^{239240} \mathrm{Pu}$ ). Figure 4-3 depicts the locations of the ambient air samplers for the Surface Environmental Surveillance Project.

A more detailed description of this program can be found in the Hanford Site Environmental Report (PNNL 1998a).

\subsection{ESTIMATED DOSES FROM DIFFUSE AND FUGITIVE EMISSIONS}

The potential dose to an offsite individual and the potential releases from diffuse and fugitive sources were estimated using ambient air monitoring data from environmental surveillance air sampling locations along the perimeter of the Hanford Site. Data from eight perimeter locations were used to perform the assessment of the 1997 emissions (PNNL 1998a, PNNL 1998b) (refer to Figure 4-3). 
Figure 4-3. Environmental Surveillance Ambient Air Sampling Locations.

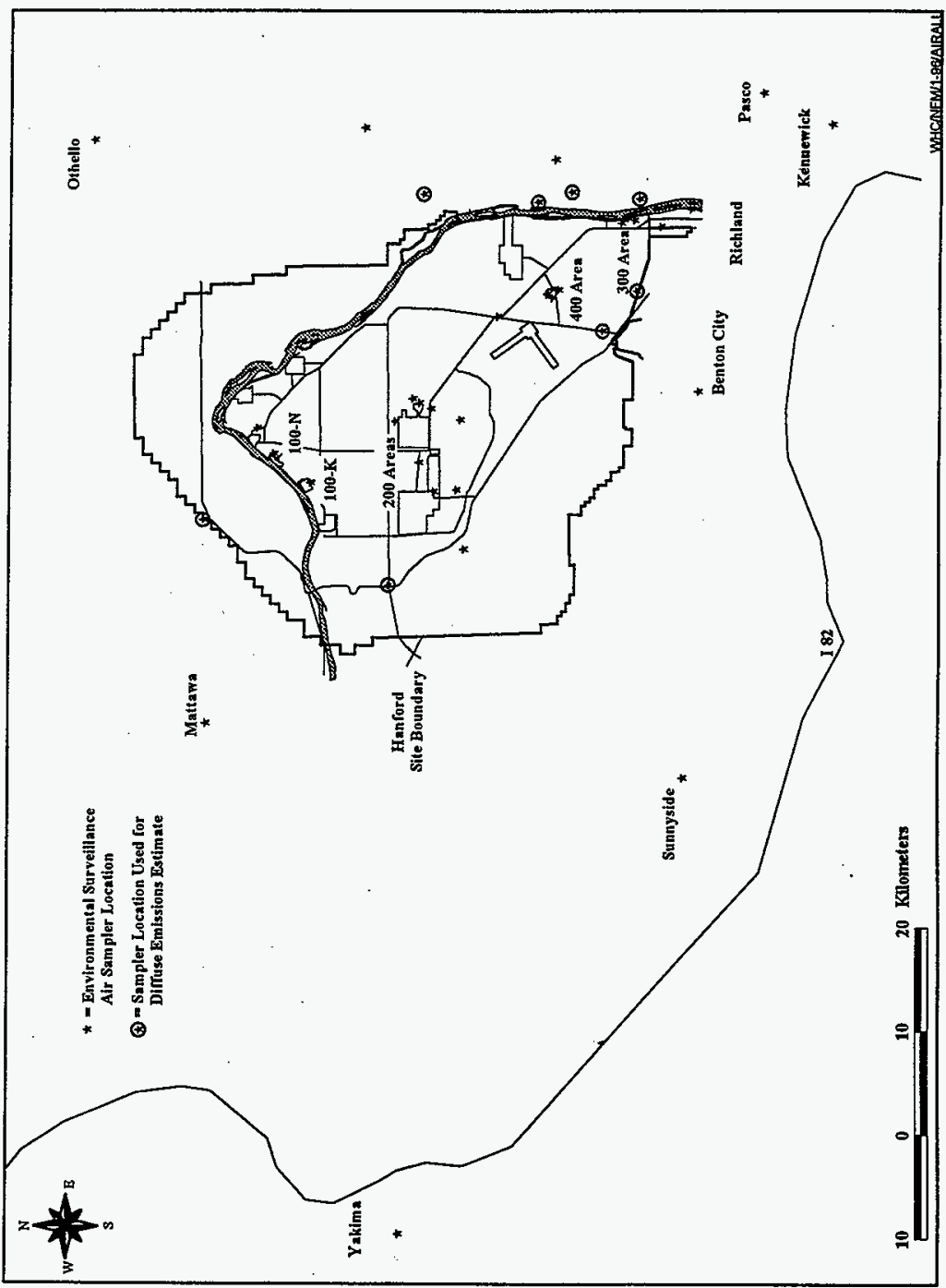




\subsubsection{Description of Dose Assessment Method}

The Hanford Site has elected to use environmental surveillance ambient air monitoring data collected at the site perimeter to estimate the dose from diffuse and fugitive emissions sources. This method is preferred for two reasons: (1) these data most accurately represent the actual exposures of an offsite individual to airborne radioactivity and (2) there is currently insufficient information about the extent and characteristics of soil contamination on the Hanford Site to use resuspension estimates in conjunction with estimating emissions from other sources of diffuse and fugitive emissions. The ambient air sampling results consisted of measured air concentrations for radionuclides that may be released from site operations and diffuse and fugitive sources. Radionuclides sampled and analyzed for include: ${ }^{3} \mathrm{H},{ }^{60} \mathrm{Co}$, ${ }^{90} \mathrm{Sr},{ }^{106} \mathrm{Ru},{ }^{125} \mathrm{Sb},{ }^{129} \mathrm{I},{ }^{134} \mathrm{Cs},{ }^{137} \mathrm{Cs},{ }^{154} \mathrm{Eu},{ }^{155} \mathrm{Eu},{ }^{234} \mathrm{U},{ }^{235} \mathrm{U},{ }^{238} \mathrm{U},{ }^{238} \mathrm{Pu}$, and ${ }^{239 / 240} \mathrm{Pu}$.

Radionuclide air concentrations resulting from 1997 stack emissions were calculated for each of the eight selected perimeter sample locations using the CAP88-PC atmospheric dispersion modelling code. The combined contributions to airborne radionuclide concentrations attributable to the stack emissions from all operating areas were subtracted from the ambient air sampling results. Averaged regional background concentrations for each radionuclide were calculated from the air sample results obtained from distant community sampling stations in Toppenish and Yakima. The average background concentration at these stations was also subtracted from the ambient monitoring results at perimeter stations, leaving the radionuclide concentrations attributable to diffuse and fugitive emissions. The only radionuclide concentrations attributable to emissions from non-DOE nuclear sites that have been subtracted were the concentrations attributable to WNP-2, because the WNP-2 release data was available at the time this report was prepared. As a result, the actual diffuse and fugitive emissions from the Hanford Site are possibly somewhat lower than the estimates presented in this report, since these estimates might contain contributions from other non-DOE nuclear sites.

Releases from diffuse and fugitive sources were estimated using the average air concentrations, attributable to diffuse and fugitive emissions, and by performing a backcalculation using CAP88-PC. The 200 West Area was assumed to be the source of all diffuse and fugitive emissions, resulting in the highest release estimates.

The estimated diffuse and fugitive emissions were used to calculate doses at selected Hanford Site perimeter locations with the CAP88-PC code. All diffuse and fugitive emissions were modeled from the 200 West Area.

\subsubsection{Results of Dose Assessment}

During 1997, the annual average ambient air concentrations at perimeter sampling stations were found to be numerically greater than the combined contributions from stack releases and background for several analytes, including ${ }^{129} \mathrm{I},{ }^{134} \mathrm{Cs},{ }^{234} \mathrm{U},{ }^{235} \mathrm{U},{ }^{238} \mathrm{U},{ }^{238} \mathrm{Pu}$, and ${ }^{239} \mathrm{Pu}$. Except for ${ }^{129} \mathrm{I}$ and ${ }^{239} \mathrm{Pu}$, these perimeter concentrations were not statistically different from those measured at the distant community stations (background locations), which are 
unaffected by Hanford Site effluents (PNNL 1998a, PNNL 1998b). Concentrations of other radionuclides might have been greater than those caused by stack releases and background radioactivity, at individual monitoring stations. However, the estimated releases, based on the corrected average air concentrations for those nuclides at all sampling stations, were less than zero.

Releases from diffuse and fugitive sources were estimated from the corrected air concentrations by performing a back-calculation using CAP88-PC. The 200 West Area was assumed to be the source of diffuse and fugitive emissions for all radionuclides, which results in the highest release estimate. The diffuse and fugitive source releases reported in Table 4-1 represent the mean of the release estimates for each of the selected perimeter locations at which a particular radionuclide was sampled. Note that not all radionuclides were evaluated at every sampling station; the estimated releases for any particular radionuclide are based on those stations where samples were analyzed for that radionuclide.

In accordance with previous guidance from DOE Headquarters; the average release estimates for diffuse and fugitive sources were calculated using air concentrations that were both positive and negative with respect to the regional background and stack emission estimates. Where the resulting mean release estimate reported in Table 4-1 for an individual radionuclide is less than zero, the average of the monitored air concentrations at the perimeter stations was numerically smaller than the combined concentrations expected as a result of stack emissions and regional background. In such cases, it is unlikely that diffuse and fugitive sources contributed significantly to the offsite measured air concentrations for those radionuclides.

The estimated diffuse or fugitive releases for the sampled radionuclides were also used to calculate the dose at the perimeter sampling stations. The Horn Rapids Road station had the highest estimated dose, and the Byers Landing station was closest to the Sagemoor Road location, which is the location of the MEI for monitored point sources on the Hanford Site. The 1997 results in Table 4-1 indicate that the hypothetical mean doses to individuals at these two locations were $3.1 \mathrm{E}-02$ and $2.2 \mathrm{E}-02 \mathrm{mrem}(3.1 \mathrm{E}-04$ and $2.2 \mathrm{E}-04 \mathrm{mSv})$, respectively. The doses for the other sampling stations, are not shown in Table 4-1, but were lower than those at the Horn Rapids Road station.

Where the release estimate for a particular radionuclide was numerically less than zero, the dose estimate for that nuclide was set equal to zero before combining the contributions of all radionuclides to obtain the total dose at each location. Although the estimated dose from diffuse and fugitive sources at the Sagemoor Road location is somewhat higher than the dose from point sources, the combined dose from point sources and diffuse and fugitive sources during 1997 was substantially below the $10 \mathrm{mrem} /$ year standard.

In addition to the sitewide diffuse and fugitive emissions estimates, diffuse and fugitive tritium emissions from two sources were estimated for 1997, to determine their potential contribution to the public radiological dose. Tritium emissions from the $100 \mathrm{~K}$ Area spent fuel storage basins were estimated at less than $2 \mathrm{Ci} / \mathrm{y}(7 \mathrm{E}+10 \mathrm{~Bq} / \mathrm{y})$, and those from the 200 Area tank farms were estimated at less than $6 \mathrm{Ci} / \mathrm{y}(2 \mathrm{E}+11 \mathrm{~Bq} / \mathrm{y})$. Emissions from 


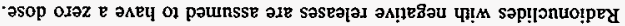

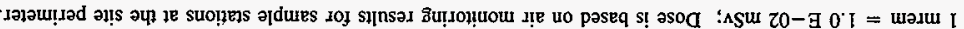

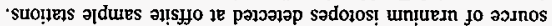

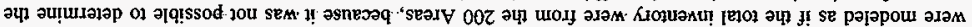

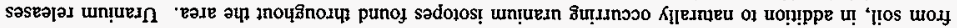

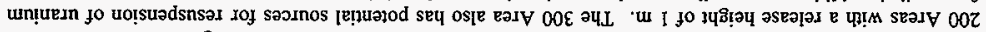

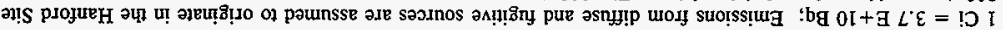
- saseajaI

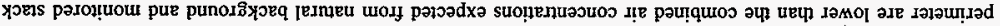

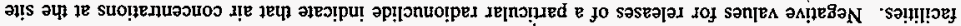

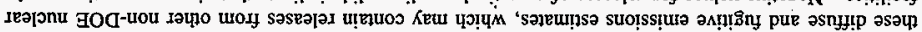

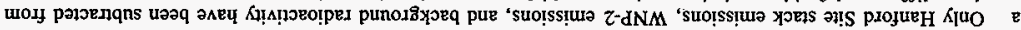

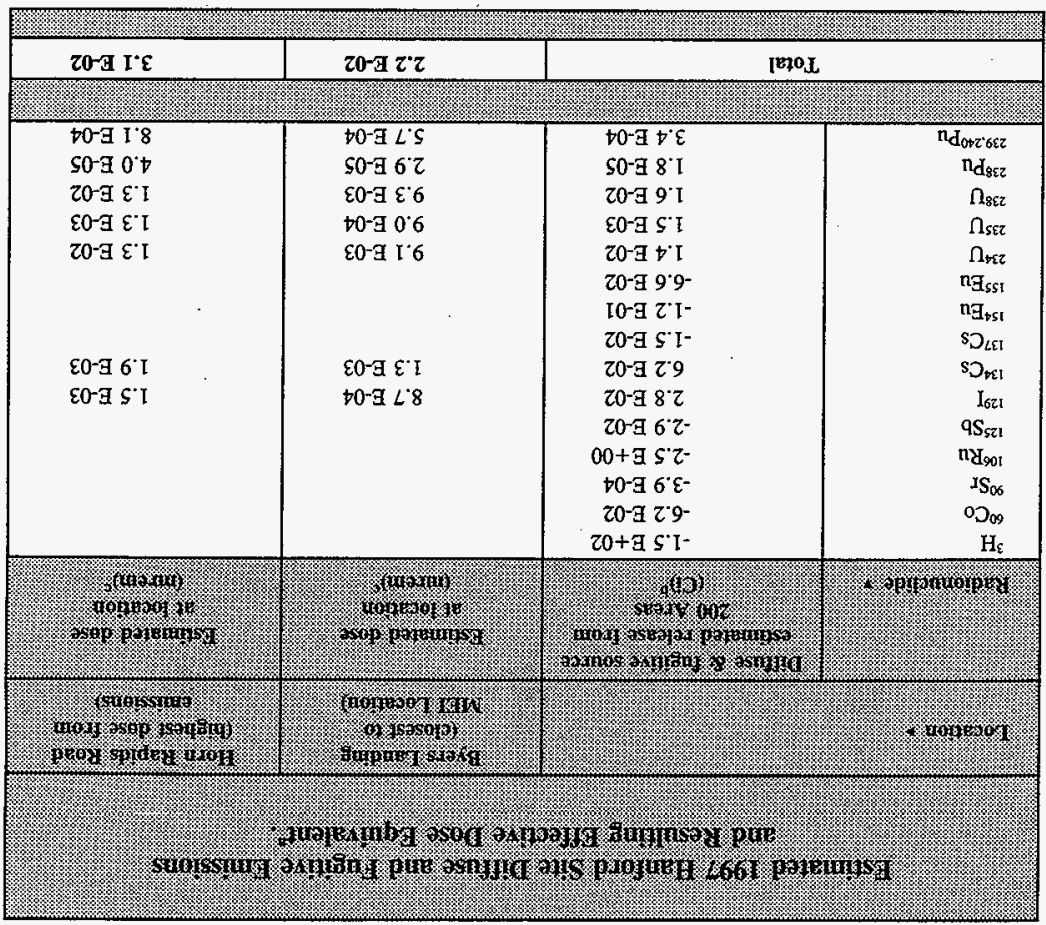

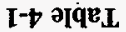


both sources were assumed to be in the form of tritiated water. During 1997, the resulting dose to the MEI at Sagemoor Road from the estimated tritium emissions was $1.3 \mathrm{E}-05 \mathrm{mrem}$ (1.3 E-07 mSv) from the $100 \mathrm{~K}$ basins, and $7.5 \mathrm{E}-05 \mathrm{mrem}(7.5 \mathrm{E}-07 \mathrm{mSv})$ from the 200 Area tank farms. The dose from these sources was much lower than the dose from monitored point source emissions and did not substantially increase the total dose to an offsite receptor.

\subsubsection{Estimate of Uncertainty in Dose Assessment}

To obtain an estimate of the uncertainty in the diffuse and fugitive emissions' dose estimates, the reported 1997 air concentrations at the Byers Landing sampling station and at the distant community stations were used to estimate the mean and $95 \%$ confidence intervals for each location. This analysis employed the uncorrected air concentrations at the reference locations, including the contributions from monitored point source releases at DOE facilities, diffuse and fugitive sources, sources other than DOE facilities, and regional background. The Byers Landing station was selected for this analysis because it is closest to the location of the Hanford Site MEI, at Sagemoor Road, and also was the only perimeter station at which all radionuclides were evaluated.

The calculation was performed using the GENII-S computer code (SNL 1992) to produce a stochastic analysis of the environmental radiation doses. The raw values of the measured air concentrations were input as basic concentrations to define an empirical distribution for each radionuclide. The code used a Latin hypercube sampling routine to select random values for each radionuclide concentration in 300 trials to obtain the dose distribution for each location. The values of parameters other than the radionuclide air concentrations were not varied as part of this analysis; therefore, the uncertainties reported in this section reflect only variability in the air sampling data.

The estimated mean dose at the Byers Landing station was 1.0 E-01 mrem $(1.0 \mathrm{E}-03 \mathrm{mSv})$ for artificially produced radionuclides sampled at that location, with a $95 \%$ confidence interval of $8.4 \mathrm{E}-02$ to $1.2 \mathrm{E}-01 \mathrm{mrem}(8.4 \mathrm{E}-04$ to $1.2 \mathrm{E}-03 \mathrm{mSv})$. The corresponding result for the distant community monitoring stations was $4.6 \mathrm{E}-02 \mathrm{mrem}$ (4.6 E-04 mSv) with $95 \%$ confidence limits of $1.7 \mathrm{E}-02$ to $7.6 \mathrm{E}-02$ mrem $(1.7 \mathrm{E}-04$ to 7.6 E-04 mSv). The mean dose estimates and the upper $95 \%$ confidence limits were higher at the site perimeter than at the distant community stations. The estimated dose at perimeter stations was largely a result of background radioactive materials (naturally occurring radionuclides and fallout). The estimated diffuse and fugitive source component was a relatively small fraction of the total offsite dose; therefore, the $95 \%$ confidence interval for the dose from diffuse and fugitive sources would be expected to be less than, or equal to, the overall dose at these locations. 


\subsubsection{Discussion}

It should be noted that the release estimates for diffuse and fugitive sources in - (Table 4-1) were obtained using the CAP88-PC, which incorporates a continuous release Gaussian plume dispersion model. Releases from diffuse and fugitive sources would be expected to occur primarily under conditions that are very different from the annual average assumptions used by CAP88-PC. This is particularly true for resuspension of contaminated soil, and to some extent for emissions from sources such as evaporation ponds, which are a function of the wind speed. Because release rates from such sources are greatest under conditions that favor atmospheric dispersion, use of an annual average continuous release model to back calculate the release quantities might introduce a significant bias in these estimates. The dose estimates for sources of this type might also be affected by seasonal variation in the resuspension rates due to prevalence of strong winds during certain seasons of the year. If these episodes occur primarily during times when crop production is minimal, some of the exposure pathways incorporated into the CAP88-PC code (direct deposition on human and animal food crops, for example) would not be applicable. The release and dose estimates reported for diffuse and fugitive sources in this evaluation should therefore be viewed as approximations whose accuracy is limited by a number of factors inherent in the sampling and modeling process.

\subsection{DIFFUSE AND FUGITIVE EMISSION SOURCES}

The Hanford Site consists of $1,450 \mathrm{~km}^{2}\left(560 \mathrm{mi}^{2}\right)$ of semiarid shrub-steppe land, of which approximately $6 \%$ has been disturbed and actively used. This $6 \%$ of land [about $83 \mathrm{~km}^{2}\left(32 \mathrm{mi}^{2}\right)$, or $8,090 \mathrm{ha}(20,000$ acres $\left.)\right]$ is divided into large operational and support areas: the 100, 200 East, 200 West, 300, 400, and 1100 Areas. The 600 Area designates the remaining land outside the operational and support areas. Almost all point, diffuse, and fugitive sources of radionuclide emissions are located in the five operational areas; one minor point source is in the 600 Area. Point source emissions are measured directly or calculated from process knowledge. Emissions from diffuse and fugitive sources are estimated using sample results from a network of environmental surveillance monitoring systerns located along the perimeter of the Hanford Site and at several receptor locations.

The Hanford Site was acquired in 1943 and dedicated to producing plutonium for national defense and managing the resulting production wastes. Restoring the Hanford Site environment is the new mission that has largely supplanted the previous operational objectives. The environmental restoration effort will entail activities such as decontaminating and decommissioning over 100 facilities and cleaning up and restoring about 1,500 waste sites. Until the restoration and cleanup work is completed, radioactive emissions may be released from hundreds of diffuse and fugitive sources, in addition to known point-source stacks.

Besides measuring point source emissions and modeling those emissions to determine public doses, environmental surveillance is conducted. Environmental and food-chain 
pathways are monitored near facilities emitting radionuclides from either point sources or diffuse and fugitive sources. The environmental pathways for all air emissions from the Hanford Site are monitored using a stratified sampling approach. Samples are collected and radiation is measured according to three surveillance zones. These zones extend from main onsite operating areas to offsite regions (PNNL 1998a).

The first zone begins near the operating facilities and ends at the Hanford Site perimeter. Diffuse and fugitive emissions generally will be most concentrated and easier to detect in this zone before diluting further as they drift offsite.

The second surveillance zone is a series of sampling stations that surround the Hanford Site near its perimeter. Because a person could live as close to the Hanford Site as some of these stations, their data represent the maximum exposures for a member of the public. Therefore, ambient air sampling data from the perimeter locations most closely reflect the actual impacts of radionuclide air emissions from point sources and diffuse and fugitive sources on the Hanford Site.

The third surveillance zone encompasses nearby and distant communities within an $80-\mathrm{km}(50-\mathrm{mi})$ radius of the center of the Hanford Site but beyond its boundaries.

Surveillance is conducted in communities to provide measurements at those locations where the most people are potentially exposed. This surveillance ensures that radionuclide levels are well below standards established to protect the public health. Finally, background concentrations are measured at distant locations and compared with onsite, perimeter, and community locations to indicate the effects of Hanford Site activities. Background locations are essentially unaffected by Hanford Site emissions but contain similar levels of radioactivity originating naturally and from nuclear-testing fallout.

The goal of environmental surveillance at the Hanford Site is to verify compliance with DOE, EPA, and WDOH radiological dose standards for public protection. This goal is accomplished by-measuring radionuclides and consequent exposure in the onsite and offsite environment. The environmental surveillance criteria are derived from: (1) the collected environmental surveillance data on radionuclides and doses; (2) applicable regulations other than DOE Orders, (3) DOE Order 5400.1; and (4) the DOE Environmental Regulatory Guide for Radiological Effluent Monitoring and Environmental Surveillance (DOE 1991). The surveillance program (PNNL 1998a) was established on these criteria and the pathway analyses that provide information on radionuclides and media contributing to human dose. Experience from Hanford Site environmental surveillance activities and studies conducted over the past 45 years has built an invaluable technical background of information for planning and data interpretation.

\subsubsection{Description of Diffuse and Fugitive Emission Sources}

The presently identified actual or potential sources of diffuse and fugitive radionuclide emissions to the environment at the Hanford Site are described in this section. Among the sources that could contribute diffuse and fugitive radionuclide emissions are several types of 
waste handling and disposal facilities, such as cribs, ponds, ditches, trenches, retention basins, valve pits, French drains, reverse wells, tanks, and burial grounds. Operating facilities or facilities on standby or that are inactive also could contribute diffuse and fugitive emissions. Deactivation, decontamination and decommissioning of facilities and cleanup of inactive waste sites could also contribute diffuse and fugitive emissions. Table 4-2 shows the numbers of and types of waste sites. Table 4-3 lists the types of diffuse and fugitive emission sources. Each site or facility usually has one or more unique features or characteristics that could contribute to the release of diffuse and fugitive emissions. Features could include passive vents, risers, equipment. and personnel access doors, and exhausters. Characteristics could include an undetected leak, unburied waste, or an absence of intrusion barriers. Rates of diffuse and fugitive emissions could be influenced by a variety of environmental conditions, such as: (1) changing atmospheric pressures; (2) wind speed; (3) erosion; (4) evaporation; (5) percolation; (6) biotic intrusion; or (7) wind-caused resuspension.

The general types of sites and facilities and their potential primary sources of diffuse and fugitive emissions are briefly described in the following sections.

4.3.1.1 Crib. Low-level liquid wastes were discharged to cribs, which are subsurface systems, similar to sanitary drain fields, that allow the liquid component of the waste to percolate into the soil. The natural properties of the soil are used to remove radioactive material from the effluent water through filtration, ion-exchange, and precipitation reactions.

Many cribs are vented to the atmosphere through vents and pipe risers. These engineered structures promote downward flow of liquids disposed in the cribs but also provide pathways to the surface and atmosphere. Secondary causes of diffuse and fugitive emissions include erosion, and uptake and intrusion by biota, followed by wind-caused suspension.

4.3.1.2 Pond. Ponds are used to manage large quantities of water (i.e., cooling water and chemical sewer wastewater) associated with chemical reprocessing operations. Normally, these liquid effluents are uncontaminated. The ponds allow percolation of the liquid effluent into the soil column. Diffuse and fugitive emissions from ponds are caused primarily by wind-caused suspension.

4.3.1.3 Ditch. A ditch is an open, unlined excavation used for disposing of liquid effluents or transporting liquid effluents to ponds for disposal. Diffuse and fugitive emissions from ditches are primarily caused by wind-caused suspension.

4.3.1.4 Trench. Early disposal practices included disposing of liquid effluents into unlined trenches and over time filling the structures with soil. These were mostly replaced by cribs such as the BC-cribs. Diffuse and fugitive emissions from trenches are primarily caused by erosion, uptake and intrusion by biota, followed by wind-caused suspension. 


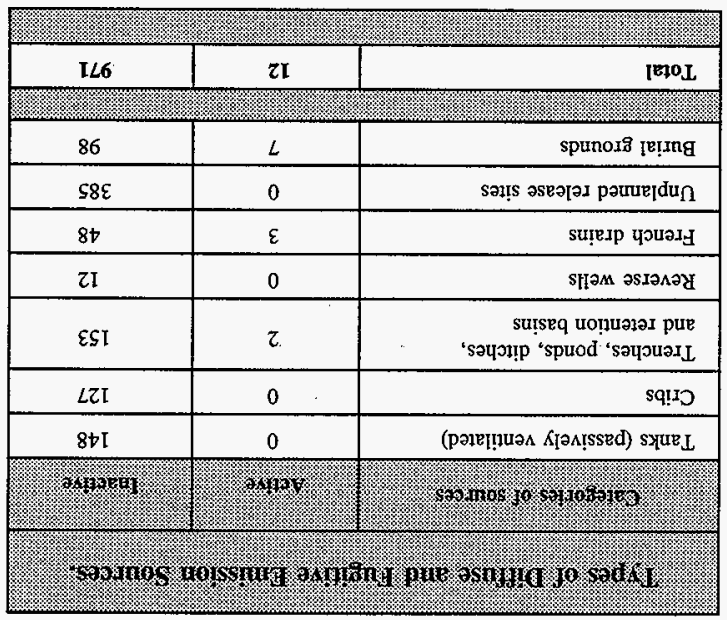

\section{$\varepsilon-t$ ગqв.}

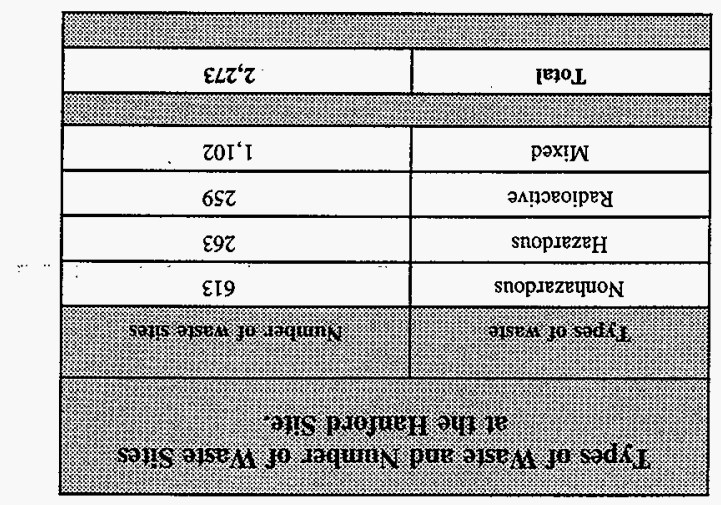


4.3.1.5 Retention Basin. Similar to trenches, retention basins generally were lined with concrete and used to hold liquids before routing them to ditches or ponds. Diffuse and fugitive emissions from retention basins are caused primarily by wind-caused suspension.

4.3.1.6 Diversion Box. A diversion box is usually an underground, concrete structure formed around a junction of transfer lines carrying liquid effluent. When diversion boxes are accessed for operations or maintenance, radioactively contaminated material might be released in the form of diffuse and fugitive emissions.

4.3.1.7 Valve Pit. A valve pit is similar in structure to a diversion box, but contains piping valves. When valve pits are accessed for maintenance or operations, radioactively contaminated material might be released in the form of diffuse and fugitive emissions.

4.3.1.8 French Drain and Reverse Well. A French drain is a rock-filled encasement inserted in the ground. A reverse well is an ordinary well used for mixing liquid waste with groundwater. These subsurface systems disposed of potentially contaminated liquid waste by promoting percolation into the soil. The natural filtration properties of the soil removed radioactive material from effluent water. Diffuse and fugitive emissions from French drains and reverse wells might occur through erosion or uptake and intrusion by biota, followed by wind-caused suspension.

4.3.1.9 Tank. A tank generally is a large reinforced metal structure that receives liquid effluent for storage. Examples are double-shell tanks (DSTs) and single shell tanks (SSTs). Pathways for diffuse and fugitive emissions from tanks include passively ventilated point sources, inactive exhausters open to the atmosphere. Transport mechanisms for these emissions include deposition and subsequent resuspension.

4.3.1.10 Burial Ground. Burial grounds are trenches in which contaminated solid waste is buried. This waste is generated by various activities on the Hanford Site. Waste packaging procedures and burial practices used depend on the type of waste. Diffuse and fugitive emissions occur at burial grounds through direct release to the atmosphere before the waste is buried, followed by erosion, uptake and intrusion by biota, and wind-caused suspension.

4.3.1.11 Deactivation, Decontamination and Decommissioning Activities. Deactivation, decontamination and decommissioning activities are being conducted to minimize the potential release or spread of contamination from facilities and equipment. Deactivation activities are intended to remove facility systems and/or areas from operational service with the intent of being ready for facility transition to either convert the facility for another use or move to permanent shutdown. Activities could include removal of fuel, draining, and/or deenergizing of systems, removal of accessible stored radioactive and hazardous material and other actions to place the facility systems and/or areas in a safe and stable condition so that a surveillance and maintenance program will be able to most cost effectively prevent any unacceptable risk to the public or the environmental until ultimate disposition of the facility. 
Decontamination consists of either physically removing contaminants or "fixing" contaminants in place to prevent mobility during demolition. Methods might include washing with water, scraping, scabbling, sandblasting, or "fixing" the contamination in place by painting, applying asphalt, etc... Demolition involves destroying and removing the structure and might include excavating any foundation. In some cases, contaminated material might be exposed to the atmosphere, but proper planning and controls minimize these exposures. In addition, monitors around demolition sites confirm that controls are effective. To date, no significant increases at demolition sites have been observed.

4.3.1.12 Waste Site Cleanup Activities. Cleanup activities are being conducted to the minimize the potential release or spread of contamination from inactive waste sites. Contaminated soils and structures are being excavated and transported to the ERDF for disposal. Contaminated materials are exposed to the atmosphere during excavation and disposal activities. Proper planning and controls such as tarps, water sprays, and fixatives are used to minimize the potential for airborne emissions. The waste sites are backfilled after excavation and the disposed material is covered with soil.

\subsubsection{Outdoor Radioactive Surface Contamination Areas. All of the following} outdoor radioactive surface contamination areas are routinely surveyed: burial grounds, cribs, trenches, retention basins, and unplanned release sites. The surveys are performed at least annually, but more frequently when needed. The magnitude, in acres, of outdoor surface contamination varies. The magnitude is not fixed because there is a continuing effort to clean, stabilize, or remediate known contaminated areas while new areas of contamination are continuing to be identified. Newly identified contamination may be because of preexisting contamination that has migrated, by way of wind or biological intrusion, to previously uncontaminated areas or because the radiological criteria have become more stringent. Eighty percent of all areas of surface contamination are estimated to have dose rates less than $1 \mathrm{mrem} / \mathrm{h}$.

Contaminated areas are posted as "Radiologically Controlled Area," "Soil Contamination Area," or "Underground Radioactive Material Area." Radiologically controlled areas are areas having a potential for an individual to receive an annual dose of up to $100 \mathrm{mrem}$. Soil contamination areas have more widespread contamination, and can have a potential for an individual to receive an annual dose of more than $100 \mathrm{mrem}$. "Underground Radioactive Material Area" signs mark cribs, burial grounds, covered trenches, and ponds, but not underground plumes that extend away from these sites. If an area has soil contamination and underground contamination, such as a surface contaminated crib, both postings will be used. The general location, by area, and the approximate area of soil contamination and underground contamination are shown in Table 4-4. Diffuse and fugitive emissions from areas of soil contamination are caused primarily by erosion, uptake and intrusion by biota, followed by wind-caused suspension. 
DOE/RL-98-33

Table 4-4

\begin{tabular}{|c|c|c|}
\hline \multicolumn{3}{|c|}{ 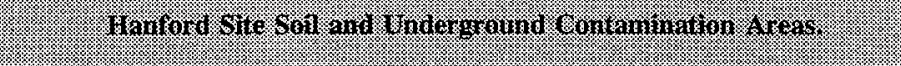 } \\
\hline 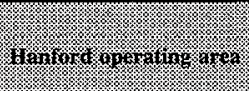 & 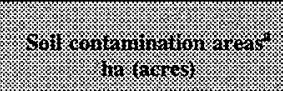 & 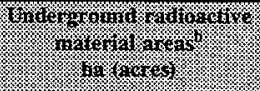 \\
\hline 100 Areas & $47(116)$ & $187(462)$ \\
\hline 200 Areas $^{\mathrm{c}}$ & $92(227)$. & 360 (889). \\
\hline 300 Area (north) & $19(47)$ & $13(32)$ \\
\hline 600 Area & $3,832(9,469)$ & $54(133)$ \\
\hline Total & $3,990(9,859)$ & $614(1,517)$ \\
\hline 3 & (1) & 2) \\
\hline
\end{tabular}

Notes:

a Inciudes areas posted as "Soil Contamination Areas" or as "Radiological Controlied Areas" and areas that have both underground and soil contamination.

b Includes areas with only underground contamination. Does not include areas that had surface and underground radioactive material.

c Includes tank farms

d $\mathrm{BC}$ controlled zone, and waste disposal facilities outside the 200 East boundary that received waste from the 200 East Area facilities (e.g. 216-A-25, 216-B-3-3, etc...), and waste disposal facilities outside the 200 West Area boundary that received waste from the 200 West Area facilities (e.g. 216-S-19, 216-U-11, etc...).

\subsubsection{Structures with Radioactive Contamination. Structures with indoor} contamination that are not actively ventilated through a point source are sources for diffuse and fugitive emissions. Many structures control diffuse and fugitive emissions with ventilation systems and contamination control practices. Ventilation systems help maintain a negative indoor air pressure, preventing airborne contaminants from leaving the building. The structures with ventilation systems discharge their air to the atmosphere via an emission control device, typically a HEPA filter. Actively ventilated point sources having a potential to emit radioactive contaminants are routinely sampled and are not considered to be a diffuse and fugitive emissions source.

The Hanford Site has many old structures with radioactive contamination and no building ventilation. Contaminants can sometimes migrate outdoors via human entry and exit. Also, contaminants can migrate outdoors via passive ventilation or animal intrusion, since these structures often have cracks and gaps that serve as pathways to the outdoors. Once the contaminants are transported outdoors, they can become airborne by wind-caused suspension. 


\subsubsection{Description of Specific Diffuse and Fugitive Emission Sources}

4.3.2.1 100 Areas' Inactive Reactor Sites. The inactive reactor sites include the: 100-B/C Area, 100-D Area, 100-F Area, and 100-H Area. The reactors are currently under surveillance and maintenance until the long-term disposition of these reactors is determined. The potential sources for diffuse and fugitive emissions included personnel and equipment passing through access doors during surveillance and maintenance.

4.3.2.2 100-K Area Basins. Two identical reactors are located in the 100-K Area. One reactor is in the 100-KE Area while the other is in the 100-KW Area. The reactors and their support facilities were constructed between 1952 and 1954, beginning service in 1955 . The 100-KW Area reactor ceased operating in 1970 and the 100-KE reactor in 1971.

The 100-KE and $-\mathrm{KW}$ reactor systems underwent D\&D after shutdown. Most of the fuel was sent to 200 East Area for processing. After the initial D\&D was completed, the fuel storage basins within the $105-\mathrm{KE}$ and $105-\mathrm{KW}$ buildings were modified to store $\mathrm{N}$ Reactor irradiated fuel. Storing this fuel began in 1975 at $105-\mathrm{KE}$ and in 1981 at $105-\mathrm{KW}$; fuel is still stored in both basins. Shipments of fuel to the basins from 100-N Area ceased in 1989.

The primary radionuclides that could be included in diffuse and fugitive emissions from the $100-\mathrm{K}$ Basins include ${ }^{3} \mathrm{H},{ }^{90} \mathrm{Sr},{ }^{137} \mathrm{Cs},{ }^{239,240} \mathrm{Pu},{ }^{241} \mathrm{Pu}$, and ${ }^{241} \mathrm{Am}$. The potential release points for diffuse and fugitive emissions include personnel and equipment access doors, which are normally closed.

4.3.2.3 $\mathrm{N}$ Reactor. The $\mathrm{N}$ Reactor was designed to produce special nuclear materials (SNM) and byproduct steam for electrical power generation. It began operations in 1963, and ceased operating in January 1987. The 100-N Area reactor complex consists of buildings for reactor operations, maintenance, support facilities, and offices. Some of the more important reactor operations facilities with radioactive materials are identified below.

The 105-N Reactor Building contains the reactor, the reactor control room, the fuel loading area, and the spent fuel storage basin. The $\mathrm{N}$ Reactor is a graphite-moderated, light-water-cooled, horizontal-pressure-tube nuclear reactor designed to produce SNM using slightly enriched uranium. Radioactively contaminated facilities associated with the N Reactor are:

- The 1301-N and 1325-N Liquid Waste Disposal Facilities consists of a crib and trench.

- The 109-N Heat-Exchanger Building transferred the heat from the primary loop to the secondary loop through steam generators.

- The 117-N Filter Building houses charcoal and HEPA filters. 
- The 107-N Basin Recirculating Building removes radionuclides from the $\mathrm{N}$ Basin Fuel Storage Facility water by filtering and demineralizing the water.

The primary radionuclides that might be included in diffuse and fugitive emissions from the $100-\mathrm{N}$ facilities include ${ }^{60} \mathrm{Co},{ }^{90} \mathrm{Sr},{ }^{137} \mathrm{Cs}$, and ${ }^{239.240} \mathrm{Pu}$. The potential primary diffuse and fugitive emission release points include railroad rollup doors, personnel and other equipment access doors (all of which are normally closed), and inactive exhaust vents and risers. A special case is the N Springs, which discharge low-level contamination (primarily ${ }^{90} \mathrm{Sr}$ ) into the Columbia River. These springs. are perhaps more closely monitored than any comparable component of the Hanford Site ecosystem. Other means of diffuse and fugitive emissions include erosion, uptake or intrusion of biota, and wind-caused resuspension.

4.3.2.4 PUREX Plant. The PUREX Plant is a collection of buildings and facilities located in the 200 East Area at the Hanford Site. The main building, 202-A, is a heavily shielded, reinforced concrete structure known as a canyon. This building contains the main equipment that was in the PUREX process of chemically separating and purifying actinides from the irradiated nuclear fuel.

Radionuclides primarily associated with the PUREX Plant include ${ }^{14} \mathrm{C},{ }^{85} \mathrm{Kr},{ }^{90} \mathrm{Sr}$, ${ }^{106} \mathrm{Ru},{ }^{129} \mathrm{I},{ }^{137} \mathrm{Cs},{ }^{147} \mathrm{Pm},{ }^{238} \mathrm{Pu},{ }^{239,240} \mathrm{Pu},{ }^{241} \mathrm{Pu}$, and ${ }^{241} \mathrm{Am}$. Potential diffuse and fugitive emission release points include access doors and the inlet-exhaust ventilation system.

4.3.2.5 $\mathrm{UO}_{3}$ Plant. The $\mathrm{UO}_{3}$ Plant, located in the 200 West Area, produced $\mathrm{UO}_{3}$ powder by calcining uranyl nitrate solutions from the PUREX Plant. The $\mathrm{UO}_{3}$ powder was sealed in steel drums for shipment offsite.

Uranium was formerly the potential primary source of radioactive diffuse and fugitive emissions from the $\mathrm{UO}_{3}$ Plant. Since the $\mathrm{UO}_{3}$ Plant's deactivation, only trace amounts of $\mathrm{UO}_{3}$ remain, all of which is sealed inside of equipment. Deactivation of the $\mathrm{UO}_{3}$ Plant has significantly reduced the potential diffuse and fugitive emissions from the facility. The noble gases radon and thoron remain as the only source of diffuse and fugitive emissions, due to residual contamination sealed inside the $\mathrm{UO}_{3}$. Plant's equipment. Potential diffuse and fugitive emission release points include access doors, all of which are restricted and controlled.

4.3.2.6 PFP. This facility is located in the 200 West Area and is used to recover, stabilize, and store plutonium. Recovered plutonium nitrate and plutonium nitrate solutions received from the PUREX Plant were reduced to plutonium dioxide. The reduction process stabilized the plutonium into the state best suited for long-term storage. PFPs mission no longer includes the production of finished plutonium metal.

Radionuclides primarily associated with PFP include ${ }^{239,240} \mathrm{Pu},{ }^{241} \mathrm{Pu}$, and ${ }^{241} \mathrm{Am}$. Potential diffuse and fugitive emission release points from PFP include access doors, passively ventilated waste drain line vents, and the inlet-exhaust ventilation system. 
4.3.2.6.1 234-5Z Building (active). The 234-5Z Building is often referred to as PFP or the 234-5 Building. The 234-5Z Building basement mostly consists of pipe tunnels carrying drain piping. The first floor houses the following: (1) two plutonium processing lines (Remote Mechanical A and Remote Mechanical C Lines) and their control rooms, (2) scrap stabilization gloveboxes, (3) plutonium storage vaults, and (4) the plutonium nitrate feed load-in/load-out (LI/LO), blending, and storage facilities.

Radionuclides primarily associated with the $234-5 \mathrm{Z}$ Building include ${ }^{239.240} \mathrm{Pu}$ and ${ }^{241} \mathrm{Am}$. Potential diffuse and fugitive release points include access doors, passively ventilated waste drain line vents, and the inlet-exhaust ventilation system.

4.3.2.6.2 236- $Z$ Building. The 236-Z Building, commonly known as PRF (Plutonium Reclamation Facility), is located south of the southeastern corner of the 234-5Z Building, connected to it by the 242-Z Building. The building air exhausts through the 291-Z-1 stack.

Radionuclides primarily associated with the $236-\mathrm{Z}$ Building include ${ }^{239,240} \mathrm{Pu}$ and ${ }^{241} \mathrm{Am}$. Potential diffuse and fugitive release points include access doors and the inlet-exhaust ventilation system.

4.3.2.6.3 232- $\mathrm{Z}$ Building. The 232- $\mathrm{Z}$ Building houses the layaway Contaminated Waste Recovery Process. It was commonly called the "Incinerator." The Contaminated Waste Recovery Process was partially decontaminated and decommissioned in 1984 and additional decontamination activities were performed in 1995.

Radionuclides primarily associated with the $232-\mathrm{Z}$ Building include ${ }^{239,240} \mathrm{Pu}$ and ${ }^{241} \mathrm{Am}$. Potential diffuse and fugitive emission release points include access doors and inlet-exhaust ventilation systems.

4.3.2.6.4 241- $\mathrm{Z}$ and $242-\mathrm{Z}$ Buildings. The $241-\mathrm{Z}$ and $242-\mathrm{Z}$ Buildings are designated as waste treatment facilities. $241-Z$ houses sumps, which are five separate enclosures or ventilated cells, each containing six tanks (5 active and 1 inactive) used to accumulate and treat the liquid wastes generated by PFP before being transferred to the tank farms. The $241-\mathrm{Z}$ is ventilated by the $296-\mathrm{Z}-3$ stack.

The 242- $\mathrm{Z}$ Building houses abandoned waste treatment process equipment, once used to recover americium. This facility was permanently shut down after a process upset in 1976 spread contamination and caused irreparable equipment damage inside. The structural integrity of the facility was not compromised, however. The facility was then decontaminated extensively before being placed in layaway pending decommissioning work. The 242-Z Building shares the main ventilation system of the 234-5Z and $236-\mathrm{Z}$ Buildings, exhausting its building air through the 291-Z-1 stack.

Radionuclides primarily associated with the $241-\mathrm{Z}$ and $242-\mathrm{Z}$ Buildings include ${ }^{239,240} \mathrm{Pu}$ and ${ }^{241} \mathrm{Am}$. Potential diffuse and fugitive emission release points include access doors and the inlet-exhaust ventilation system. 
4.3.2.6.5 2736-ZB Building. The 2736-ZB shipping area and receiving area both accommodate a maximum of 100 shipping containers, each of which is about the size of a 55-gal (210-L) drum. Adequate spacing is provided between containers to meet criticality prevention requirements, personnel exposure specifications, and corridor access standards to emergency staging areas. The two areas are physically separated by a wall.

Radionuclides primarily associated with the $2736-\mathrm{ZB}$ Building include ${ }^{239.240} \mathrm{Pu}$ and ${ }^{241} \mathrm{Am}$. Potential diffuse and fugitive emission release points include access doors and the inlet-exhaust ventilation system.

4.3.2.6.6 216-Z-20 Crib Effluent Facility. The 216-Z-20 Crib was constructed and commissioned for use in September 1981 to dispose of waste water previously discharged to the 216-U-10 Pond through the 216-Z-19 Ditch. Use of the crib allowed the radioactively contaminated 216-Z-19 Ditch and 216-U-10 Pond to be decommissioned.

The 216-Z-20 Crib was designed and constructed to dispose of potentially radioactive liquid effluents from the 231-Z, 232-Z, 236-Z, 242-Z, 291-Z, 234-5Z, and 2736-ZB Buildings. The same building effluent headers and piping that served the 216-Z-19 Ditch were used. These headers and piping contain some residual contamination. The 207-Z Seepage Basin, later renamed 216-Z-21 Seepage Basin, was designed and constructed to dispose of uncontaminated water from the storm sewer north of the 234-5Z Building, water-tank overflow, and swamp-cooler waste water. The 216-Z-20 Crib and the 216-Z-21 Seepage Basin are inactive and are no longer used to dispose of liquid effluents and waste water.

The $216-\mathrm{Z}-20 \mathrm{Crib}$ is approximately $460 \mathrm{~m}(1,500 \mathrm{ft})$ long with a cross section at the bottom that is approximately $3 \mathrm{~m}$ (10 ft) wide. Gravel was used as backfill to distribute effluents throughout the crib. A vapor barrier was placed above the gravel backfill. Soil was placed over the top to bring the area back to the surrounding grade.

Radionuclides primarily associated with the $216-\mathrm{Z}-20$ Crib include ${ }^{239,240} \mathrm{Pu}$ and ${ }^{241} \mathrm{Am}$. Diffuse and fugitive emissions could be released by way of vents, risers, erosion, biotic uptake and intrusion, and wind-caused resuspension.

4.3.2.7 T Plant. Originally, $T$ Plant was a fuel separations facility using the bismuth phosphate process. Now the facility is used for radioactive decontamination and repair of equipment. This facility currently is active.

T Plant is located in the 200 West Area of the Hanford Site. Buildings, structures, or special facilities included as part of this facility are the 221-T and 2706-T Buildings, deactivated 221-T Building Head-End, and 214-T Chemical Storage Building. Ancillary buildings, structures, and areas included are the 271-T, 291-T and 221-TA Buildings, and the 211-T Chemical Storage Area. Decontamination processes are conducted in the 221-T and 2706-T Buildings. The 221-T Building Head-End was used for special experiments and operations. The 214-T Building stores chemicals. The 211-T Chemical Storage Area consists of a permitted pad that stores nonradioactive hazardous waste. The 271-T Building 
provides office space to staff supporting $T$ Plant operations. The 291-T Building houses the exhaust ventilation fans for the 291-T-1 main stack. The 221-TA Building houses the supply ventilation fans for the 221-T Building canyon.

Radioactive decontamination activities are performed in the canyon area. The canyon area consists of 37 cells and one railroad tunnel door. The railroad tunnel (used for transporting equipment into and out of the canyon, as well as for some decontamination) enters the plant at cell $2 \mathrm{~L}$. A motor-driven rolling steel door, provides railroad canyon access.

Primary radionuclides associated with T Plant include ${ }^{90} \mathrm{Sr},{ }^{137} \mathrm{Cs}$, and ${ }^{239.240} \mathrm{Pu}$. Potential diffuse and fugitive emission release points include access doors and the inlet-exhaust ventilation system.

4.3.2.7.1 2706-T Building. The 2706-T Building is a ground-level structure constructed of prefabricated steel. It has two openings on the west end that are fitted with roll-up metal doors that allow access to the pit areas. Low-level radioactive decontamination activities are performed in these two pits.

Radionuclides primarily associated with the $2706-\mathrm{T}$ Building include ${ }^{90} \mathrm{Sr},{ }^{137} \mathrm{Cs}$, ${ }^{239,240} \mathrm{Pu}$, and ${ }^{241} \mathrm{Am}$. Potential diffuse and fugitive emission release points include access doors, the inlet-exhaust ventilation system, and an outdoor storage area.

4.3.2.7.2 221-T Building - Head-End Operations. The 221-T Building head-end consists of a canyon area extending from the basement floor to the roof. This canyon area has several deck levels and a parapet wall. Four floor levels adjacent to the canyon house include: (1) an electrical switchgear room, (2) a chemistry laboratory, (3) office areas, (4) a change room, (5) a lunch room, (6) a control room, (7) an instrument shop, (8) a maintenance shop, (9) and storage areas. In 1994, flow from eight process sewer lines originating in the Head-End was discharged to the 216-T-1 Ditch, which is no longer in use.

Radionuclides primarily associated with 221 -T Head-End Operations include ${ }^{90} \mathrm{Sr}$, ${ }^{137} \mathrm{Cs}$, and ${ }^{239.240} \mathrm{Pu}$. Potential diffuse and fugitive emission release points include access doors and inlet-exhaust ventilation system.

\subsection{1-T Building - Pressurized-Water (Cooled) Reactor Fuel Assembly} Storage. Canyon cell $2 \mathrm{R}$ has been modified to store approximately 76 pressurized-water reactor Core 2 blanket fuel assemblies that were used to power the Shippingport Reactor. Provisions have been made to store the assemblies at the Hanford Site for up to 20 years.

The 221-T Building galleries are maintained at atmospheric pressure, while the 221-T Building canyon area is maintained at a negative pressure with respect to atmosphere. A primary design feature of these systems is to ensure that potentially contaminated canyon air is completely separate from the clean air in the 221-T Building galleries and the 271-T Building, which is the office space connected to the 221-T Building. In addition, the canyon air system is operated at negative pressures with respect to the other systems. 
The 271-T Building is adjacent to the 221-T Operating Gallery. While most of this building is used for office space, portions are used by T-Plant Operations.

Radionuclides primarily associated with 221-T-PWR Fuel Assembly Storage include ${ }^{90} \mathrm{Sr},{ }^{137} \mathrm{Cs}$, and ${ }^{239,240} \mathrm{Pu}$. Potential diffuse and fugitive emission release points include access doors and the inlet-exhaust ventilation system.

4.3.2.8 224-T Transuranic Waste Storage and Assay Facility. The 224-T TRUSAF was originally used to purify plutonium nitrate by the lanthanum fluoride process. $\therefore$ After phaseout of the bismuth phosphate plants, the lanthanum fluoride process was no longer needed and the facility remained inactive until the early 1970's. At that time, the 224-T Building was modified for storage of plutonium scrap in liquid and solid forms. In 1984 the facility was selected to house the transuranic waste storage and assay (TRUSAF) operation, in which transuranic waste containers were nondestructively tested and stored for eventual shipment to the Waste Isolation Pilot Plant (WIPP). All stored transuranic wastes were removed from TRUSAF by September 1997, and nondestructive examination and nondestructive assay operations at 224-T TRUSAF were terminated. Currently, no waste is stored and no waste operations are performed at 224-T TRUSAF.

Radionuclides primarily associated with $224-\mathrm{T}$ TRUSAF include ${ }^{90} \mathrm{Sr},{ }^{137} \mathrm{Cs}$, and ${ }^{239,240} \mathrm{Pu}$. Potential diffuse and fugitive emission release points include access doors and the inlet-exhaust ventilation system.

4.3.2.9 B Plant. B Plant was originally designed to chemically process spent nuclear fuels. Radiological containment and confinement features were incorporated in the various facilities and support systems to prevent exposure of plant personnel and the general public to excessive radiation. The plant was then modified to separate strontium and cesium from the fission product waste stream following plutonium and uranium recovery from irradiated reactor fuels in the PUREX Plant. The recovered purified and concentrated strontium and cesium solutions were then transferred to the Waste Encapsulation Storage Facility (WESF) for conversion to solid compounds, encapsulation, and interim storage. After strontium and cesium removal, the remaining waste was transferred from B Plant to the Tank Farms. There are currently no production activities at B Plant, but several systems are operating systems to perform the current B Plant mission. B Plant receives and stores various chemicals.

B Plant consists of three main buildings. They are the 221-B Processing Building and the 271-B Service and Office Building. The 221-B Process Building and its attached 271-B Service Building were constructed in 1943.

Radionuclides primarily associated with B Plant include ${ }^{90} \mathrm{Sr}$ and ${ }^{137} \mathrm{Cs}$. Potential diffuse and fugitive emission release points include access doors and the inlet-exhaust ventilation system. 
4.3.2.10 Waste Encapsulation and Storage Facility (WESF). WESF, the 225-B Building, is an operating facility used to ensure safe storage and management of the cesium and strontium capsules. Construction of the WESF was completed in 1974.

Radionuclides primarily associated with WESF include ${ }^{90} \mathrm{Sr}$ and ${ }^{137} \mathrm{Cs}$. Potential diffuse and fugitive emission release points include access doors and the inlet-exhaust ventilation system.

4.3.2.11 222-S Laboratory Complex. The 222-S Laboratory Complex is located in the southeast corner of the 200 West Area. The facility is composed of the main laboratory complex (222-S) and a number of ancillary buildings and structures.

4.3.2.11.1 222-S Laboratory. The 222-S Laboratory is a two-story, aboveground building with a subterranean service level. This building is divided into laboratory support spaces, office spaces, a multi-curie wing, eleven environmental hot cells, and supplemental service areas. The building is designed with its own waste disposal facility, decontamination facility, fire protection and alarm system, ventilation sýstem, and radiation monitoring systems.

The 222-S Laboratory Annex houses the maintenance shop, instrument shop, and the counting room filter building.

Radionuclides primarily associated with the 222 -S Laboratory include ${ }^{90} \mathrm{Sr}$ and ${ }^{137} \mathrm{Cs}$. Potential diffuse and fugitive emission release points include access doors and the inlet-exhaust ventilation system.

4.3.2.11.2 222-SA Laboratory. The 222-SA Laboratory is a five-wide modular building located southeast of the 222-S Laboratory. Part of this laboratory prepares nonradioactive standards for Hanford Site laboratories. The other section of the laboratory is used for cold-process development work and standards preparation. The 222-SA Laboratory only has the potential for nonradioactive diffuse and fugitive emissions.

4.3.2.11.3 2716-S Storage Building. The 2716-S Storage Building, located south of the 222-S Laboratory, is partitioned off for the storage of acids and bases. It provides both long- and short-term storage capability for laboratory materials and contains no radioactive materials.

There are no radioactive diffuse fugitive emissions from the 2716-S Storage Building.

4.3.2.11.4 207-SL Retention Basin. The 207-SL Retention Basin acts as a temporary holding facility for potentially radioactive or hazardous liquid effluents before being transferred by means of a cross-site pipeline to the Treated Effluent Disposal Facility, located in the 200 East Area. Waste water from the laboratory, normally free of radioactive and hazardous chemical contamination, is routed to the 207-SL Retention Basin. This facility is a covered, below-grade concrete structure, directly east of the 222-S Building. Two 25,000-gal (95,000-L) compartments allow batch collection, sampling, and discharge of the 
waste. Three 20,000 -gal $(75,708-\mathrm{L})$ storage tanks were added in 1994 to improve waste transfer and storage capabilities.

Radionuclides primarily associated with the $207-$ SL Retention Basin include ${ }^{90} \mathrm{Sr},{ }^{137} \mathrm{Cs}$, and ${ }^{239 / 240} \mathrm{Pu}$. Potential diffuse fugitive release points include access doors and seams in the basin cover blocks.

4.3.2.11.5 219-S Waste Handling Facility. The 219-S Waste Handling Facility collects liquid waste generated by the 222-S Laboratory operations that is contaminated radioactively and/or with hazardous chemicals. This facility consists of two below-grade vaults (A and $\mathrm{B}$, also called cells) containing; three stainless-steel tanks, a Transite building, the pipe trench and operating gallery, and an attached concrete-walled sample gallery. Tanks TK-101 and TK-102 are in vault A and tanks TK-103 and TK-104 are in vault B.

Radionuclides primarily associated with the 219-S Waste Handling Facility include ${ }^{90} \mathrm{Sr}$ and ${ }^{137} \mathrm{Cs}$. Potential diffuse fugitive release points include access doors and seams in the vault cover blocks.

4.3.2.11.6 222-SB Filter Building. The 222-SB Filter Building, located south of the 222-S Building, houses 96 HEPA filters which provide final filtration for the 222-S Laboratory. Under normal operation of the ventilation system, three electrically powered fans exhaust air from the 222-S Laboratory. Exhaust air leaves the 222-S Building through the 296-S-21 stack. Emergency exhaust ventilation is provided by the 222-SE Filter Building, in the event that two or more exhaust fans fail to operate.

Radionuclides primarily associated with the $222-\mathrm{SB}$ Filter Building include ${ }^{90} \mathrm{Sr}$ and ${ }^{137} \mathrm{Cs}$. Potential diffuse fugitive emission release points include access doors and the inlet-exhaust ventilation system.

4.3.2.11.7 222-SC Filter Building. The 222-SC Filter Building, located north of the 222-S Laboratory, contains the second and third stage HEPA filtration for hot cells 1-A, 1-E-1, 1-E-2, 1-F, and 11-A-1 through 11-A-6. The hot cells in rooms 1-A, 1-E, 1-E, 1-F, and 11-A are serviced by the main building supply and exhaust ventilation. The 222-SC Filter Building houses five parallel pairs of HEPA filters, which provide filtration to hot cell exhaust air before it enters the main exhaust plenum and final filtering in the 222-SB Filter Building. A total of four stages of HEPA filtration are provided for the hot cell ventilation exhaust.

Radionuclides primarily associated with the 222-SC Filter Building include ${ }^{90} \mathrm{Sr}$ and ${ }^{137} \mathrm{Cs}$. Potential diffuse and fugitive emission release points include access doors and the inlet-exhaust ventilation system.

4.3.2.11.8 222-SE Filter Building. The 222-SE Filter Building, located south of the 222-S Building, is a building that houses 56 HEPA filters. This building provides redundant backup filtering capabilities for the 222-\$ Laboratory exhaust. 
Radionuclides primarily associated with the 222-SE Filter Building include ${ }^{90} \mathrm{Sr}$ and ${ }^{137} \mathrm{Cs}$. Potential diffuse and fugitive emission release points include access doors and the inlet-exhaust ventilation system.

4.3.2.11.9 218-W-7 Dry Waste Burial Vault. The 218-W-7 Dry Waste Burial Vault is located $40 \mathrm{ft}$ southeast of $222-\mathrm{S}$. This underground concrete vault was removed from service around 1960 . It was used primarily for disposal of plutonium-contaminated dry-hood waste generated by the 222-S Laboratory. Access to the tank is through a locked hatchway.

Plutonium-239 and -240 are the radionuclides primarily associated with the 218-W-7 Dry Waste Burial Vault. A locked access hatchway is the only potential release point for diffuse and fugitive emissions.

4.3.2.11.10 216-S-26 Crib. The 216-S-26 Crib is no longer in service and the pipeline to the crib has been sealed off. All waste water collected in the 207-SL Retention Basins is now discharged to the Treated Effluent Disposal Facility, located in the 200 East Area. Additionally, the 222-SA waste water has been rerouted from the crib to the 207-SL Retention Basins. The line from the 291-S Stack Fan House, which formerly discharged steam condensate to the crib, has been isolated.

Radionuclides historically discharged to the $216-\mathrm{S}-26 \mathrm{Crib}$ include ${ }^{90} \mathrm{Sr},{ }^{137} \mathrm{Cs}$, and ${ }^{239,240} \mathrm{Pu}$. Diffuse and fugitive emissions may be released by way of vents, risers, erosion, biotic uptake and intrusion, and wind-caused resuspension.

4.3.2.12 Waste Verification and Sampling Facility. The Waste Verification and Sampling Facility, the 213-W Building, is located in the 200 West Area of the Hanford Site. The 213-W Building is adjacent to the 272-WA Building (the Operations Support Building) at the 218-W-5 Burial. Grounds at the west end of the 200 West Area. Systems ancillary to the 213-W Building also are included.

The primary function or process associated with the Facility is the verification of waste drums received from waste generators. Because of limited use, it is was transferred to the 200 West Tank Farms Facility in 1995.

Radionuclides primarily associated with the $213-\mathrm{W}$ Building include ${ }^{90} \mathrm{Sr}$ and ${ }^{137} \mathrm{Cs}$; low-level waste is present also. Potential diffuse and fugitive emission release points include access doors and the inlet-exhaust ventilation system.

4.3.2.13 Central Waste Complex. The Central Waste Complex (CWC) is a group of structures located on the west side of 200 West Area exclusion zone. The primary function or process associated with the CWC Facility is the receipt and storage of radioactive and mixed waste (MW), which is currently ongoing. This facility has the potential to generate radioactive and/or hazardous chemical emissions and radioactive and/or hazardous chemical liquid effluent. 
Radionuclides associated with the Central Waste Complex are from a wide group of mixed fission, mixed waste, and transuranic radionuclides. Potential diffuse and fugitive emission release points include access doors and the inlet-exhaust ventilation system. The facility consists of the following.

4.3.2.13.1 Pu and PCB Storage Facility. This facility is a steel building with concrete curbing within its perimeter. The building has a water-based fire suppression system and continuous air monitors (CAM) for detection of particulate airborne radionuclides.

4.3.2.13.2 Low-Flashpoint MW Storage Modules. Each of the low-flashpoint MW storage modules has a catch sump with a holding capacity of 750 gal $(2,800 \mathrm{~L})$. Modules used for storage of TRU-mixed waste also have draft ventilation.

4.3.2.13.3 Mixed-Waste Storage Facilities. The Buildings 2402-WB through 2402-WL, 2402-W, and 2403-W are metal structures with concrete floors, ventilation systems, and water-based fire protection. The concrete floors have provisions to collect any spilled liquids. The facilities have CAMs for detection of particulate airborne radionuclides.

4.3.2.13.4 Mixed-Waste Storage Pad. The MW Storage Pad is a $9,000 \mathrm{ft}^{2}\left(320 \mathrm{~m}^{3)}\right.$ concrete storage pad with $6-\mathrm{in}$. $(15-\mathrm{cm})$ curbing around its perimeter. The pad has a stormwater collection system that allows for disposing of the stormwater after sampling and analysis.

4.3.2.13.5 Waste Receiving and Storage Pad. The Waste Receiving and Storage Pad is a 61-m-by-46-m (200-ft-by-150-ft) asphalt pad.

4.3.2.14 Tank Farms. Liquid waste from chemical processing operations containing high concentrations of radionuclides is stored on an interim basis in underground tanks. The Hanford-Site tank farms contain 177 tanks (149 SSTs and 28 DSTs) with capacities ranging from 50,000 to 1.2 million gal ( 190,000 to 4.5 million L). Since 1967 , newly generated liquid waste has been stored in DSTs. The SSTs are no longer receiving waste.

The location of all the tank farms is the 200 East and 200 West Areas. Both DSTs and SSTs are present in these areas. Tank farms in the 200 East Area include the A, AX, B, BX, BY, C (SSTs), AN, AZ, AY, AP, and AW (DSTs). Those in the 200 West Area are the S, SX, T, TX, TY, U (SSTs) and the SY (DST).

Hanford Site tank farms comprise transfer routes, diversion boxes, storage vaults, double-contained receiver tanks (DCRT), and evaporators.

A system of underground pipes is used to transfer wastes from the 200 East Area waste generators to the DSTs, between the DSTs, and from the DSTs to treatment and storage units in the 200 East and 200 West Areas. 
Radionuclides primarily associated with the tank farms include ${ }^{3} \mathrm{H},{ }^{14} \mathrm{C},{ }^{90} \mathrm{Sr},{ }^{106} \mathrm{Ru}$, ${ }^{125} \mathrm{Sb},{ }^{137} \mathrm{Cs},{ }^{239,240} \mathrm{Pu}$, and ${ }^{241} \mathrm{Am}$. Diffuse and fugitive emission release locations may include vents, risers, access hole covers, inlet-exhaust ventilation systems, diversion boxes, transfer lines, and storage vaults.

4.3.2.14.1 Double-Shell Waste Tanks. The DSTs are of two distinctly different types. The capacity of the first type is 1 to 1.2 million gal ( 3.79 to 4.54 million $\mathrm{L}$ ), and is designed for long-term storage (up to 50 years) of high-activity mixed waste.

Twenty-four 1.2-million-gal (4.5-million-L) non-aging .waste DSTs and four 1.0-million-gal (3.8-million-L) aging waste DSTs have been built. For efficiency during construction and operation, the 1-million-gal tanks were grouped into six tank farms.

The second type of DST is smaller, with storage capacities ranging from 800 to 45,000 gal $(3,028$ to $170,370 \mathrm{~L})$. These tanks were used primarily for lag storage of waste before transfer to the larger tanks or to other facilities. These smaller tanks are called DCRTs.

All DSTs were fabricated as three concentric tanks. Waste is stored in the freestanding primary tank. The secondary tank sits on a concrete pad. The completely enclosed annulus serves as a containment barrier if the primary tank should leak. The annulus is ventilated and continually monitored for evidence of primary tank leakage. The third tank is a concrete shell that encloses the sides of both primary and secondary tanks for additional containment, radiation shielding, and structural support.

Ancillary equipment also is present, such as transfer lines between tank farms and DCRTs, valve pits, diversion boxes, and tank-farm piping.

4.3.2.15 242-A Evaporator. The 242-A Evaporator complex is located in the 200 East Area of the Hanford Site. The 242-A Building is located south of the 241-A and 241-AX Tank Farms and north of the 242-AW Tank Farm. The 242-A Building contains the evaporator vessel and supporting process equipment. The building ventilation exhaust fans and HEPA filter housings are located on the south side of the building. An emergency diesel generator is located on the south side of the building. Raw water, steam, and electrical power are provided to the 242-A Building from existing service facilities in the 200 East Area.

In general, the 242-A Evaporator Facility can be divided into three areas: process, service, and operating. The process area includes: the evaporator room, pump room, condenser room, and ion exchange enclosure. The service area includes: the aqueous make-up (AMU) room, loadout and hot equipment storage room, loading room, and HVAC room. The operating areas include: the control room, personnel change rooms, lunchroom, office, and storage rooms.

Radionuclides primarily associated with 242 -A include ${ }^{3} \mathrm{H},{ }^{14} \mathrm{C},{ }^{90} \mathrm{Sr},{ }^{106} \mathrm{Ru},{ }^{125} \mathrm{Sb},{ }^{137} \mathrm{Cs}$, ${ }^{239,240} \mathrm{Pu}$, and ${ }^{241} \mathrm{Am}$. Potential diffuse and fugitive emission release points include access doors and the inlet-exhaust ventilation system. 
4.3.2.16 242-S Evaporator. The 242-S Evaporator, currently on standby, is located in the 200 West Area, west of the 241-SY Tank Farm. It consists of an evaporator vessel and supporting process equipment. The building ventilation exhaust fans and HEPA filter housings are on the south side of the building. An emergency diesel generator is on the south side of the building. Raw water, steam, and electrical power are provided from existing service facilities in the 200 East Area. There are three main areas: process, service, and operations. The process area includes: the evaporator room, pump room, condenser room, and ion-exchange enclosure. The service area includes: the Aqueous Make-up (AMU) room, loadout and hot equipment storage room, loading room, and the HVAC room. The operating areas include: the control room, personnel change rooms, lunchroom, offices, and storage rooms.

Radionuclides primarily associated with $242-\mathrm{S}$ include ${ }^{14} \mathrm{C},{ }^{90} \mathrm{Sr},{ }^{125} \mathrm{Sb},{ }^{129} \mathrm{I},{ }^{137} \mathrm{Cs}$, ${ }^{239.240} \mathrm{Pu}$, and ${ }^{241} \mathrm{Am}$. Potential diffuse and fugitive emission release points include access doors and the inlet-exhaust ventilation system.

4.3.2.17 242-T Evaporator. The 242-T Evaporator, currently inactive, is located in the 200 West Area, east of the 241-TX and 241-TY Tank Farms. The 242-T Evaporator Facility is divided into a processing area and a control area. The process area includes the 242-T building, the 242-TA Vault, and 242-TB Ventilation building. The control area is contained in the metal building adjacent to the east wall of the 242-T building.

The 242-T Building is a steel reinforced concrete structure 48 feet in length, 42 feet wide, and 23 feet high. The building contains the Condensate Area, the Feed Cell, and the Evaporator Area. The Feed Cell houses the 4,000 gallon B-1 Blend Tank plus the interconnecting piping between this tank, the evaporator vessel, and the 241-TX Tank Farm. The Evaporator Area (called the hot cell) contains the evaporator vessel, a cyclone separator, the cyclone separator catch tank, two evaporator feed preheaters, a floor sump, and interconnecting piping between the feed and cold cells and the TX Tank Farm. The Condensate Area (called the cold cell) contains two 4,000 gallon condensate catch tanks, a scrubber, condenser, floor sump, and interconnecting piping between the feed and hot cells and the TX Tank Farm.

The 242-TA Vault is a concrete lined pit with a ground level steel cover. Inside this vault sits the 4,000. gallon R-1 Receiver Tank and the piping connecting this tank to the feed cell - acetic high level waste from Z Plant flowed into this tank for pumpage to the feed cell.

The 242-TB Ventilation Building contains the ventilation equipment and instruments for the TB ventilation system. This ventilation system services the R-1 Receiver Tank and the TA Vault.

4.3.2.18 Grout Treatment Facility (GTF). The GTF combined low-level radioactive liquid waste with a cement mixture that is pumped into disposal vaults. The GTF was placed in cold standby in 1993. 
Radioactive constituents primarily associated with GTF include ${ }^{90} \mathrm{Sr},{ }^{137} \mathrm{Cs}$, and other low-level radioactive waste from the tank farms. Potential diffuse and fugitive emission release modes include vents, risers, and the inlet-exhaust ventilation system.

4.3.2.19 Low-Level Burial Grounds. The active low-level radioactive solid waste burial grounds are located in the 200 Areas of the Hanford Site. The active sites to be considered are those addressed in the Part A, Dangerous Waste Permit Application for the Low-Level Burial Grounds (DOE-RL 1988). In the 200 West Area, the burial grounds to be considered include 218-W-5, 218-W-6, 218-W-3A, 218-W-3AE, 218-W-4B, and 218-W-4C.: In the 200 East Area, the burial grounds to be considered include 218-E-10 and 218-E-12B.

The entire array of low-level radionuclides from operations on the Hanford Site is associated with the Low-Level Burial Grounds. Diffuse and fugitive emissions may be released by way of open burials (before covering), ruptured containment vessels, biotic intrusion, erosion, and wind-caused resuspension.

4.3.2.19.1 Burial Ground 218-W-3A. Burial Ground 218-W-3A began receiving waste in 1970. It consists of 61 trenches covering 50.3 acres (20.4 hectares). Waste stored or disposed of in this burial ground includes mixed, transuranic, low-level, and retrievable waste. Other types of waste include ion-exchange resins and industrial waste (such as failed equipment, tanks, pumps, ovens, agitators, heaters, hoods, jumpers, vehicles, and accessories). This burial ground used to store spent fuel.

4.3.2.19.2 Burial Ground 218-W-3AE. Burial Ground 218-W-3AE began receiving waste in 1981. It consists of 31 trenches covering 49.4 acres (20 hectares). Waste in this burial ground includes low-level and mixed waste. Other types of waste placed in this burial ground include rags, paper, rubber gloves, disposable supplies, broken tools, and industrial waste.

4.3.2.19.3 Burial Ground 218-W-4B. Burial Ground 218-W-4B began receiving waste in 1968. It consists of 13 trenches and 12 caissons covering 8.6 acres ( 3.5 hectares). The trenches contain mixed and retrievable TRU waste and were filled before 1980 . Caisson Alpha 4 is believed to contain mixed waste.

4.3.2.19.4 Burial Ground 218-W-4C. Burial Ground 218-W-4C began receiving waste in 1978. It consists of 69 trenches covering 51.7 acres (20.9 hectares). Some of the waste in this facility is TRU, mixed, and low-level. Other types of waste placed in these trenches include contaminated soil, decommissioned pumps, pressure vessels and hardware, and stored spent fuel. Some of the trenches are designed for retrievable storage of waste.

4.3.2.19.5 Burial Ground 218-W-5. Burial Ground 218-W-5 began receiving waste in 1986. It consists of 35 trenches (with room to expand to 57 trenches) covering 84.0 acres (34 hectares). The trenches contain low-level mixed waste that includes lead bricks and shielding. Low-level waste also was placed in these trenches. 
4.3.2.19.6 Burial Ground 218-W-6. Burial Ground 218-W-6 has not received any waste to date. When developed, this burial ground will consist of 35 trenches and cover approximately 44.5 acres ( 18 hectares).

4.3.2.19.7 Burial Ground 218-E-10. Burial Ground 218-E-10 began receiving waste in 1960. It consists of 18 trenches covering 56.7 acres (22.9 hectares). Waste was received from PUREX, B Plant, and N Reactor. The waste includes low-level and low-level mixed waste such as dragoff waste, failed equipment, and industrial waste.

4.3.2.19.8 Burial Ground 218-E-12B. Burial Ground 218-E-12B began receiving wastes in 1967. It consists of 94 trenches covering about 173 acres (70 hectares). Areas are set aside for future expansion of this burial ground. Some of the waste contained in this burial ground is TRU, mixed, and low-level. Trench 94 contains decommissioned U.S. Navy submarine reactor compartments.

4.3.2.20 340 Complex. The 340 Complex is located in the 300 Area of the Hanford Site near the Columbia River. The 340 Complex was constructed to collect, store, and transport radioactive liquid wastes from 300 Area facilities. The 324, 325, 326, 327, and 329 Buildings are connected to the Radioactive Liquid Waste System (RLWS) which empties directly into the 340 vault tanks. The 326 and 329 Buildings' drains have been isolated from the RLWS. Direct shipments of containers or tankers are also received at the 340 Facility and added to the vault tanks. The 340 Complex also houses the 300 Area Retention Process Sewer (RPS), which collects process wastewater with the potential to become radioactively contaminated. This waste stream is accumulated in the 307 Basins. This waste stream is monitored for radioactive materials and can be transferred to the 340 vault tanks if necessary. After sampling and analysis in the 307 Basins, the wastewater is pumped to the process sewer for treatment at the 300 Area Treated Effluent Disposal Facility.

Uranium, ${ }^{60} \mathrm{Co},{ }^{90} \mathrm{Sr},{ }^{137} \mathrm{Cs},{ }^{238} \mathrm{Pu},{ }^{239 / 240} \mathrm{Pu}$, and ${ }^{241} \mathrm{Am}$ are the most significant radionuclides potentially present at the 340 Complex. Potential diffuse and fugitive emission release points include access doors and the inlet-exhaust ventilation system and the 307 Retention Basin.

4.3.2.20.1 340-A Building. The 340-A Building houses six above ground storage tanks which provide temporary storage of radioactive liquid waste. The 340-NT-EX Stack powered exhaust system provides airborne ventilation to the storage tanks, and the 340-A Building air is passively ventilated to the atmosphere via a roof air vent.

Smearable radiological contamination resulting from leakage of radioactive liquid waste from the storage tanks has been detected in the 340-A Building. The radionuclides with the most significant dosimetric impact include ${ }^{90} \mathrm{Sr},{ }^{137} \mathrm{Cs},{ }^{238} \mathrm{Pu},{ }^{239 / 240} \mathrm{Pu}$, and ${ }^{241} \mathrm{Am}$, which could potentially be present in diffuse and fugitive emissions from the facility.

4.3.2.20.2 340-B East Building. The 340-B East Building serves as a railroad car loadout facility. This facility houses railroad cars during the transfer of radioactive liquid wastes from the 340 tanks to the railroad cars. 
During periods of time when the railroad cars are not housed in the 340-B East Building, the facility's exhaust system is not operated, thus the building is not maintained at negative pressure. Consequently, diffuse and fugitive emissions can potentially occur. Potential diffuse and fugitive emission release points from the 340-B East Building include personnel and equipment access doors. The radionuclides with the largest dosimetric impact that could be potentially released as diffuse and fugitive emissions include: ${ }^{90} \mathrm{Sr},{ }^{137} \mathrm{Cs},{ }^{238} \mathrm{Pu}$, ${ }^{239 / 240} \mathrm{Pu}$, and ${ }^{241} \mathrm{Am}$.

4.3.2.20.3 Retention Process Sewer. The Retention Process Sewer:consists of a plumbing system, a liquid waste retention/storage system, and a monitoring system. The plumbing system consists of underground single-walled pipes, which transport liquid wastes from the generating facilities to the liquid waste retention/storage system. The retention system consists of four lined and connected open-top concrete basins, which are also known as the 307 Retention Basins. Each basin has a holding capacity. of $95,000 \mathrm{~L}(25,099$ gal). The monitoring system utilizes in-line gamma radiation detectors to monitor radiation levels in Retention Process Sewer's liquids. The gamma detectors are used to divert liquids to the 340 Facility's Radioactive Liquid Waste System when predetermined levels are exceeded. Composite samples of 307-Basin influent are collected and analyzed prior to release of the Basin contents. If alpha contamination is detected above specified action levels, the wastewater can be isolated and pumped to the 340 Vault's tanks for shipment to an onsite TSD facility.

4.3.2.20.4 340 Vault. The 340 Vault houses two 15,000 gallon radioactive mixed tanks that accumulate mixed waste for shipment to the 200 Area TSD facilities. The 340 vault, the vault tanks, and the associated piping system is ventilated through the 340-NT-EX stack. Smearable and fixed contamination resulting from tank overfills and maintenance activities are documented in facility records.

4.3.2.21 300 Area Fuel Supply Shutdown (FSS) Facility. The 300 Area FSS Facility was previously known as N Reactor Fuels Fabrication Facility, which was changed to reflect its current mission. The facility is located in the 300 Area of the Hanford Site. It consists of 16 buildings, two tank farms, and associated pipe trenches and drains. The structures are located on the north side of the 300 Area. The 300 Area Fuels Fabrication facilities began operation in 1944 with some structures being added in the 1950's and 1960's. The following sections provide information on each of the facilities. The 300 Area Fuel Fabrication Facility buildings have been decontaminated in preparation for transferring facility management responsibilities to the ERC. 
The 300 Area FSS Facilities and Current Function/Activity include:

Building
303-A
$303-\mathrm{B}$
$303-\mathrm{E}$
$303-\mathrm{G}$
3712
3716
313
333
$303-\mathrm{K} / 3707-\mathrm{G}$
$303-\mathrm{F}$
$304 / 304-\mathrm{A}$
$334-\mathrm{A}$
334
$303-\mathrm{M}$

Waste Transfer Inactive waste acid transfer system pipe trenches within and between Systems 333, 334-A, 334-TF, 313, 303-F and 311-TF facilities

311-TF

334-TF

\section{Current Function/Activity}

Uranium fuel element storage

Uranium billet storage

Uranium fuel element storage (empty)

Uranium billet storage

Finished uranium fuel.element. and billet storage

Unfinished uranium fuel element storage

Inactive fuels fabrication support facility

Inactive fuel manufacturing

Inactive radioactive material and waste storage (303-K) and change room (3707-G)

Inactive neutralized waste acid pump house

Inactive uranium concretion facility (304) and change room (304-A)

Inactive waste acid storage and transfer system (RCRA closure)

Inactive process sewer monitoring system

Uranium oxide facility (inactive)

Inactive neutralized waste acid storage and transfer facility (adjacent to $303-\mathrm{F}$ building)

Inactive product and waste acid storage facility (adjacent to the 334 building)

A significant unirradiated uranium inventory, present at the time the $\mathrm{N}$ Reactor standby was announced, is stored in buildings 303-A, 303-B, 303-G, 3712, and 3716, at the 300 Area FSS Facility . A portion of the uranium is in the form of extrusion billets. The balance is in the form of unenriched fuel elements. Some of the stored fuel elements are partially processed elements, recovered at the time fabrication ceased.

4.3.2.21.1 333 Building. The primary N Reactor Fuels Fabrication activities were conducted in the 333 Building. The fuel fabrication operation produced $N$ Reactor fuel from 1961 until 1987 when operations were stopped.

This facility used a variety of mechanical, chemical, and electrical processes to convert uranium billets and assorted components into finished fuel assemblies for irradiation at N Reactor.

Operations and resulting radionuclide air emissions in the 333 Building ceased in January 1987. Emission monitoring equipment was shut down at that time. Process liquid effluents are no longer generated. However, air conditioning water and stormwater continue . to be discharged from the building by way of the process sewer. 
Uranium and ${ }^{99} \mathrm{Tc}$ are the most significant radionuclides in surface contamination at the 333 Building. The occasional operation of the heating and cooling ventilation system results in potential diffuse and fugitive emissions from equipment and personnel access doors. Normally, ventilation systems are shutdown, with the exception of comfort heating and cooling in the office areas. Consequently, infiltration and exfiltration induced by wind and ambient pressure changes results in potential diffuse and fugitive emissions from the building. However, on-going surveillance activities within the building indicate that radioactive contamination is not present, in a dispersible form.

4.3.2.21.2 313 Building. The south end of the building houses: the uranium laboratory, copper casting, waste-acid treatment facility, engineering development laboratory, training rooms, and office areas, all of which have been shutdown and are no longer occupied. The North end of the 313 Building houses a complete N Reactor pressure tube fabrication facility consisting of a 4000 ton Sutton extrusion press, draw bench, grinders, autoclaves, inspection equipment, and chemical cleaning equipment, none of which was ever used for their intended purpose. The Hanford Metal Working process equipment has been sold to a commercial company, and the north section of the 313 Building has been leased for the commercial operation of the extrusion equipment.

The 313 Building was used from 1944 to 1971 for fabricating uranium fuel elements for the eight retired, single-pass reactors in the 100 Areas. The function of the building changed when those reactors ceased operating. The building then was used as an $\mathrm{N}$ Fuels Fabrication Support Facility to treat uranium-bearing acid in the waste-acid treatment system. The recovered uranium sludge was recycled to form new uranium billets at Fernald, Ohio. Engineering development laboratory work and continuous monitoring of airborne emissions was terminated in 1991.

Uranium and ${ }^{99} \mathrm{Tc}$ are the potential primary radioactive constituents in diffuse and fugitive emissions from the 313 Building. Heating and cooling ventilation system in the southern portions of the building are no longer in operation. Infiltration and exfiltration, induced by wind and ambient pressure changes, results in potential diffuse and fugitive emission release points from equipment and personnel access doors.

4.3.2.21.3 303-K/3707-G Radioactive Mixed Waste Storage Facility. The 303-K Facility was designed and constructed in 1943. The facility is a concrete-block building with a poured concrete ceiling. Outside, the storage area consists of two concrete pads, two asphalt pads, and a gravel area. The north room of the 303-K Facility originally had one roof exhaust fan. The fan was used from 1953 to 1977 while aluminum spacer and equipment decontaminating was done. The roof vent fan was replaced with a HEPA-filtered exhaust system in 1977, which was used until the fall of 1982. The exhaust system was only turned on at the end of the curing operation for the concreted billets of recyclable scrap uranium chips and fines, or if hydrogen levels indicated a billet fire had occurred. The HEPA exhaust system has not operated since the concrete curing operation was discontinued in 1982. 3707-G is a small adjoining building used as a change room. The 3707-G Building 
also serves as an entry point into portions of the $303-\mathrm{K}$ Building, the adjacent outdoor fenced area, and the concrete pad area.

These buildings no longer discharges either air emissions or liquid effluents to the environment. It was used to store radioactive and mixed waste until 1994. Only small quantities of non dispersible radioactive contamination remain in the buildings.

Uranium and ${ }^{99} \mathrm{Tc}$ are the primary radioactive constituents in potential diffuse and fugitive emissions from the 303-K Facility. Infiltration and exfiltration, induced by wind and ambient pressure changes, results in potential diffuse and fugitive emissions from equipment and personnel access doors.

4.3.2.21.4 303-M Uranium Oxide Facility. The 303-M Uranium Oxide Facility is adjacent to the 333 Building and consists of one building, an adjacent outdoor drum storage area, and a small process sewer filter building. The 303-M Uranium Oxide Facility stored and treated recycled material generated during the $\mathrm{N}$ Reactor fuel fabrication processes. The fuel fabrication waste material consisted of pyrophoric saw fines and lathe turnings, known as chips, composed of slightly enriched uranium and Zircaloy-2. The wastes were calcinated to remove their pyrophoric properties, eliminating the possibility of spontaneous combustion during transportation.

The 303-M Uranium Oxide Facility operated from 1983 to 1987 for the calcination of the wastes generated. The facility ceased operating in 1987.

During operation, the facility operated under negative pressure and the building exhaust was released to the atmosphere through a single stack. The emissions were controlled with a bag-house and HEPA filters.

The 303-M Building is currently closed. The water and electricity have been shut off and the exhaust stack capped. The primary radionuclides associated with the facility are uranium and ${ }^{99} \mathrm{Tc}$.

4.3.2.21.5 304/304-A Uranium Concretion Facility. The 304 Building was designed and constructed in 1952 as a pilot plant for the lead-dip canning process. The 304's uranium concretion equipment was installed in 1971 with a drainage trench and sump to remove liquids resulting from spills, leaks, and daily operations. Standard spill-response procedures inside the building included washing the spilled waste to the sump where the fines would settle out. The waste water was drained into the 300 Area process sewer, and the fines were shoveled from the sump and concreted. Additionally, an outside storage area is on the north side of the facility. The storage area is a concrete pad surrounded by asphalt. The 304-A Building is change room addition to the 304 Building.

The building has no inlet air supply system, the water is shut off, and drains have been plugged. The exhaust stack was capped in 1994 . Uranium and ${ }^{99} \mathrm{Tc}$ are the primary radionuclides potentially present in diffuse and fugitive emissions from personnel and equipment access doors. 
4.3.2.21.6 311 Tank Farm. The recoverable and nonrecoverable uranium-bearing neutralized waste solutions from the 313 Building were combined in aboveground tanks (tanks 40 and 50) in the 311 Tank Farm. These liquid wastes eventually were transferred to tanker trucks and disposed of in the 183-H Basins or the 200 Areas. After 1988, if the wastes were below radioactive release limits, they were shipped to offsite contractors for treatment, storage, and/or disposal. The two tanks received approximately 420,000 gal (1.6 million L) of waste each year, during periods of peak production.

In addition to waste tanks 40 and 50 , the 311 Tank Farm-has a 4,000-gal $(15,000-\mathrm{L})$ tank that was used to store nitric acid and two 10,000 -gal $(38,000-\mathrm{L})$ tanks that were used to store sodium hydroxide. The tanks were emptied in 1991 and no longer contain nitric acid or sodium hydroxide.

Uranium and ${ }^{99} \mathrm{Tc}$ are the potential primary radioactive constituents in diffuse and fugitive emissions from the 311 Tank Farm. Diffuse and fugitive emissions may be released by way of uranium-bearing material transfers and unplanned releases.

4.3.2.21.7 334-A Waste Acid Storage Building. The 334-A Waste Acid Storage Building was completed in late 1974 and placed in use in January 1975 . For four months in 1973, an underground tank and tank 4 in the 334 Tank Farm were used to collect acid waste that would be transferred to the 313 Building for neutralization. The underground tank began to leak in August 1973 and was removed during construction of the 334-A Building. The waste acids were discharged directly into the process sewer until the 334-A Building was built in December 1974. The tanks in the 334-A building received approximately 210,000 gal $(790,000 \mathrm{~L})$ of waste acids per year. These waste acids consisted of hydrofluoric, nitric, and sulfuric acids with copper, zirconium, chromium, and uranium in solution. Following storage, the acids were pumped from the 334-A Building to the south end of the 313 Building for neutralization.

The building heating and cooling air handling systems have been shut down, the water and electricity have been shut off, and drains have been plugged. The storage tanks in the building have been isolated and cleaned. Uranium and ${ }^{99} \mathrm{Tc}$ are the potential primary radioactive constituent in diffuse and fugitive emissions from the 334-A Building. The potential diffuse and fugitive emission release points include personnel and equipment access doors.

4.3.2.21.8 303-A, 303-B, and 303-G Uranium Fuel Storage Buildings. The fuel stored in the 303-A, 303-B and 303-G buildings includes unused fuel elements, uranium billets and some irradiated fuel elements, which had been loaded into N Reactor. The fuel elements that had been loaded into $\mathrm{N}$ Reactor, were recovered during reactor defueling with only low levels of surface contamination on them. These fuel elements are appropriately packaged for safe handling and storage at their present storage location. The structures are single story concrete block and cement construction, with only three doors and no windows. The roofs are precast concrete slabs covered with felt, tar, and gravel. There are four $25 \mathrm{~cm}$ (10 in.) diameter holes in the walls at floor level for water drainage in the unlikely event of a sprinkler system discharge. The buildings are unheated and are not provided with active 
ventilation. The buildings are equipped with automatic fire detection and sprinkler (dry) systems with freeze protection in the valve rooms. Infiltration and exfiltration, induced by wind and ambient pressure changes, results in potential diffuse and fugitive emissions from equipment and personnel access doors.

4.3.2.21.9 303-E Uranium Fuel Storage Building. No fuel is currently stored in the 303-E building. Until 1996, the 303-E building had been used to store unirradiated fuel, like the 303-A, 303-B, and 303-G Buildings, until the building's fuel inventory was removed. Only small quantities of radiological contamination remain in the building. Infiltration and exfiltration, induced by wind and ambient pressure changes, results in potential diffuse and fugitive emissions from equipment and personnel access doors.

4.3.2.21.10 3712 Uranium Fuel Storage Building. The 3712 Building is a one-story steel-frame structure with metal panel siding, metal panel roof, and a concrete floor and foundation. The building is used to store uranium billets, finished fuel elements, uranium/ scrap and standards, and unfinished fuel pieces. A heating, ventilation, and air conditioning (HVAC) unit provides heating/cooling of the south portion of the building, when occupied. Infiltration and exfiltration, induced by wind and ambient pressure changes, results in potential diffuse and fugitive emissions from equipment and personnel access doors.

4.3.2.21.11 3716 Uranium Fuel Storage Building. The 3716 Building is a single-story building with insulated aluminum siding and roof. The building is used to store unfinished fuel pieces, which are capped with plastic caps and kept in wooden boxes. The steam heating system and evaporative cooler for the building have been shutdown. Infiltration and exfiltration, induced by wind and ambient pressure changes, results in potential diffuse and fugitive emissions from equipment and personnel access doors.

4.3.2.21.12 Waste Transfer System: The waste transfer system serves as an inter-facility pipe chase between the 333 building, 334-A building, 334 tank farm, 313 building, 303-F building, and the 311 Tank farm. It is comprised of various sized concrete trenches topped with removable reinforced concrete or steel panels. Due to historic spills, there are some portions of the system that contain small quantities of radioactive contamination. There is no ventilation system associated with the pipe chase. Infiltration and exfiltration, induced by wind and ambient pressure changes, results in potential diffuse and fugitive emissions from crevices between the pipe chase covers.

4.3.2.22 Fast Flux Test Facility (FFTF). The FFTF is a 400-megawatt-thermal, sodium-cooled, fast-neutron-flux reactor designed specifically for irradiation testing of nuclear reactor fuels and materials for liquid-metal, fast-breeder reactors. The reactor is capable of extensive in-core irradiation testing, including eight core positions that may be used with independent instrumentation for the test loops. In addition to irradiation-testing capabilities, FFTF is designed for long-term testing and evaluation of plant components and systems for liquid-metal, fast-breeder reactors. 
The FFTF is a complex of buildings and equipment arranged around the reactor containment building. This arrangement includes the reactor, equipment, and structures for heat removal, containment, core component handling and examination, instrumentation and control, and buildings for supplying utilities and other essential services. The reactor is currently shut down.

Radionuclides primarily associated with FFTF include ${ }^{3} \mathrm{H}$ and ${ }^{137} \mathrm{Cs}$. The potential primary diffuse and fugitive emission release points include the personnel and equipment access doors and the inlet-exhaust ventilation system. 
DOE/RL-98-33

\subsection{SUPPLEMENTAL INFORMATION}

This section has supplemental information related to Hanford Site radionuclide air emissions in 1997. The information consists of:

- Population dose estimate

- Compliance status with Subparts Q and T of 40 CFR 61

- Discussion on ${ }^{220} \mathrm{Rn}$ emissions

- Emission points subject to monitoring requirements of 40 CFR 61.93(b) and costs to upgrade noncompliant monitoring systems

- Emissions estimates and confirmatory measurement data for specific NOCs

- Status of quality assurance (QA) program intended to comply with description in 40 CFR 61, Appendix B, Method 114.

\subsection{POPULATION DOSE}

The regional population dose (collective EDE) from 1997 Hanford Site air emissions was estimated by calculating the radiation dose to the population of approximately 376,000 residing within an $80 \mathrm{~km}(50 \mathrm{mi})$ radius of the onsite operating areas (PNL 1991). Pathways of exposure to the population from releases of radionuclides to the atmosphere include inhalation, air submersion, ground-shine, and consumption of food. Population exposure to radionuclide air emissions was determined using values of population-weighted atmospheric dispersion factors for distance and each compass sector. The collective EDE (using the GENII computer code, [PNL 1988]) from air emissions to the population within $80 \mathrm{~km}$ $(50 \mathrm{mi})$ of the Hanford Site during 1997 was $4.9 \mathrm{E}-02$ person-rem (4.9 E-04 person-Sv). Releases to surface water added another $9.8 \mathrm{E}-01$ person-rem (9.8 E-03 person-Sv) so the population dose from all Hanford Site radionuclide air and liquid effluents was $1.5 \mathrm{E}-01$ person-rem (1.5 E-03 person-Sv) in 1997.

\subsection{COMPLIANCE STATUS WITH 40 CFR 61, SUBPARTS Q AND T}

In 40 CFR 61, Subpart Q, "National Emission Standards for Radon Emissions From Department of Energy Facilities," paragraph 61.190 states that the provisions of Subpart $Q$ apply to the design and operation of all storage and disposal facilities for radium-bearing material that emit ${ }^{222} \mathrm{Rn}$ to the air. Paragraph $61.191(\mathrm{~b})$ states that a source means any building, structure, pile, impoundment, or area used for interim storage or disposal that is or 
contains waste material containing radium in sufficient concentration to emit ${ }^{222} \mathrm{Rn}$ in excess of the $20 \mathrm{pCi} / \mathrm{m}^{2} / \mathrm{s}$. On the Hanford Site, no storage and disposal facilities for radium-bearing waste materials are established. Therefore, the provisions of this subpart are not applicable to the Hanford Site.

Activities at the Hanford Site were evaluated for compliance with 40 CFR 61 Subpart T, "National Emissions Standards for Radon Emissions From the Disposal of Uranium Mill Tailings". In paragraph 61.220, "Designation of Facilities," owners and operators of all sites used for the disposal of tailings and that managed residual radioactive material or uranium byproduct materials during and following the processing of uranium ores and that are listed in or designated by the Secretary of Energy under Title I of the Uranium Mill Tailings Control Act of 1978 or regulated under Title II of that act are subject to the provisions of the subpart. On the Hanford Site, uranium milling and uranium-ore processing activities are not conducted. Therefore, the provisions of this subpart are not applicable to the Hanford Site.

\subsection{EMISSION OF ${ }^{220}$ Rn AND ${ }^{222}$ Rn FROM TECHNOLOGICALLY ENHANCED SOURCES}

${ }^{220} \mathrm{Rn}$ and ${ }^{222} \mathrm{Rn}$ are radioactive noble gases that pass unimpeded through the HEPA filters and sample filters. Technologically enhanced sources of ${ }^{220} \mathrm{Rn}$ and ${ }^{222} \mathrm{Rn}$ existed at the 327 Building. However, the 327 Building's potential emissions of ${ }^{220} \mathrm{Rn}$ and ${ }^{222} \mathrm{Rn}$ were below threshold values that would require monitoring for these radionuclides.

During 1997, emissions monitoring for ${ }^{220} \mathrm{Rn}$ and ${ }^{222} \mathrm{Rn}$, at the 327 Building, was performed above the minimum regulatory requirements. Radon emissions and doses for 1997 are being reported separately from other point source emissions since monitoring was not required per state and federal regulations. These doses have been included in the effective dose equivalent reported for WAC 246-247 compliance, since these emissions were monitored. It is currently Hanford's policy to include all emissions measured in this report.

The ${ }^{220} \mathrm{Rn}$ released from the 327 Building originated from a ${ }^{228} \mathrm{Th}$ source. Releases from this facility are listed in Table $2-1$. The resulting ${ }^{220} \mathrm{Rn}$ dose was $1.9 \mathrm{E}-03 \mathrm{mrem}$ (1.9 E-05 mSv) to the MEI at the Sagemoor Road farm (refer to Table 3-3). The calculation was performed assuming that all ${ }^{220} \mathrm{Rn}$ was converted to its first long-lived decay product, ${ }^{212} \mathrm{~Pb}$, before reaching the receptor. This was necessary because CAP88 does not account for ingrowth of decay products for the short-lived ${ }^{220} \mathrm{Rn}$ radionuclide.

The ${ }^{222} \mathrm{Rn}$ emissions from the 327 Building added another $6.4 \mathrm{E}-04 \mathrm{mrem}$ (6.4 E $-06 \mathrm{mSv}$ ) to the $\mathrm{MEI}$ dose (refer to Table 3-3). 


\subsection{EMISSIONS ESTIMATES AND CONFIRMATORY MEASUREMENT DATA FOR SPECIFIC NOCS}

This section contains the emissions estimates and periodic confirmatory measurement data as required by specific Notices of Construction (NOCs) and other regulatory agreements.

\subsubsection{NOC for Portable Temporary Radioactive Airborne Emissions Units (PTRAEU)}

The Radioactive Air Emissions Notice of Construction Portable/Temporary Radioactive Air Emission Units (DOE-RL 1997b) requires that the estimated emissions from these units be summarized in the annual Radionuclide Air Emissions Report for the Hanford Site. The information provided in Table 5-1 fulfills this requirement. The estimated emissions are obtained from Section 10.2 of the NOC. When documentation demonstrates that the handling limits for these emission units have not been exceeded, the estimated emissions would be equal to or less than the values provided in Section 10.2 of the NOC.

\section{Table 5-1}

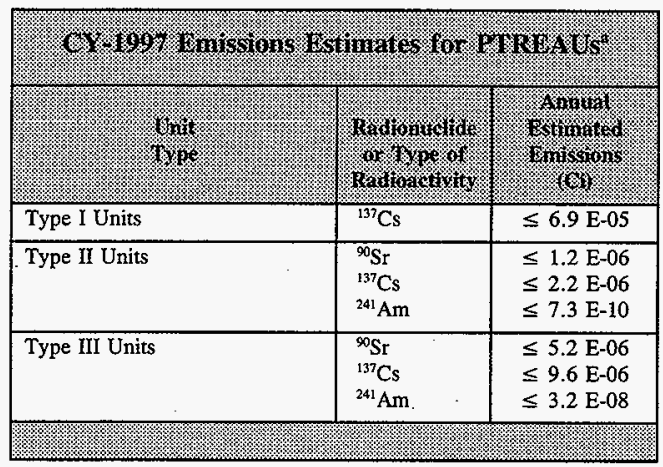

Note:

a $1 \mathrm{Ci}=3.7 \mathrm{E}+10 \mathrm{~Bq}$. 


\subsubsection{NOCs for the 309-RTF-EX Stack}

The estimated emissions for the 309-RTF-EX stack are provided in Table 5-2. These emissions estimates include emissions from all NOCs associated with this point source.

Table 5-2

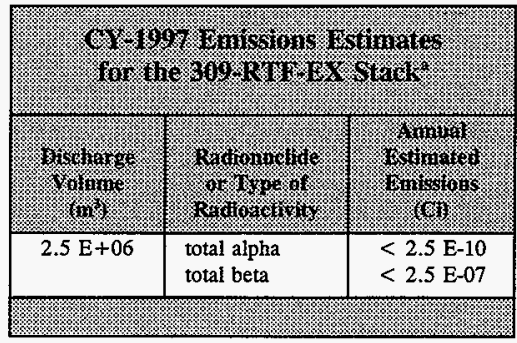

Note:

a $1 \mathrm{Ci}=3.7 \mathrm{E}+10 \mathrm{~Bq}$.

\subsubsection{Release Estimates for the Containment Tent Exhausters at Tank Farm Facilities}

Potential emissions from containment tent exhauster use in Tank Farms during calendar year 1997 are provided in the following. From available sample data, potential emissions are computed (Table 5-3).

$3.2 \mathrm{E}-05$ Ci total beta

1.4 E-08 Ci total alpha

From maximum concentration values used together with the computed exhaust volumes, potential emissions are:

$1.312 \mathrm{E}+05 * 1.2 \mathrm{E}-08=1.6 \mathrm{E}-03 \mathrm{Ci}$ total beta

$1.312 \mathrm{E}+05 * 5.5 \mathrm{E}-12=7.2 \mathrm{E}-07 \mathrm{Ci}$ total alpha

Therefore, potential emissions using air sample data are between:

$1.6 \mathrm{E}-03$ and $3.2 \mathrm{E}-05 \mathrm{Ci}$ total beta

7.2 E-07 and 1.4 E-08 Ci total alpha

Potential emissions using HEPA smear data are:

$3.5 \mathrm{E}+04 \mathrm{dpm} /\left(100 \mathrm{~cm}^{2}\right) * 1 \mathrm{Ci} /(2.22 \mathrm{E}+12 \mathrm{dpm}) * 3,716 \mathrm{~cm}^{2}=5.9 \mathrm{E}-07 \mathrm{Ci}$ total beta 
$2.0 \mathrm{E}+01 \mathrm{dpm} /\left(100 \mathrm{~cm}^{2}\right) * 1 \mathrm{Ci} /(2.22 \mathrm{E}+12 \mathrm{dpm}) * 3,716 \mathrm{~cm}^{2}=3.3 \mathrm{E}-10 \mathrm{Ci}$ total alpha

The results were calculated as follows:

The HEPA filter is a standard HEPA with dimensions of 24 in $\times 24$ in $\times 11.5$ in $(61 \mathrm{~cm} \times 61 \mathrm{~m} \mathrm{x} 29 \mathrm{~cm})$.

Contamination was assumed to be uniformly distributed over only the surface of the HEPA.

The smear was taken by first removing the circular elephant trunk connected to the entrance to the HEPA housing. This trunk also holds a pre-filter. The smear was taken off the entrance surface of the HEPA filter.

The surface of the HEPA filter was calculated be $3,716 \mathrm{~cm}^{2}\left(576.0 \mathrm{in}^{2}\right)$. The contamination smears represent $100 \mathrm{~cm}^{2}\left(15.5 \mathrm{in}^{2}\right)$. The HEPA filter surface could contain 37.2 times the quantity on the smear.

Assuming emissions are from contamination smear data are everything on the inlet surface of the HEPA filter, the emission calculation equation is as follows:

$\mathrm{Sd} * 1 \mathrm{Ci} /(2.22 \mathrm{E}+12 \mathrm{dpm}) * 3,716 \mathrm{~cm}^{2}=$ Curies released.

where,

$\mathrm{Sd}=$ the smear data, $\mathrm{dpm} / 100 \mathrm{~cm}^{2}$.

Emissions from the air sample data were computed by simply multiplying the sample data by the total exhaust volume. This is possible because:

$\mu \mathrm{Ci} / \mathrm{ml}=\mathrm{Ci} / \mathrm{m}^{3}$.

Table 5-4 contains containment tent exhauster data, for the exhausters used in Tank Farms during 1997. This information is being reported in accordance to the conditions specified by WDOH. 
Table 5-3

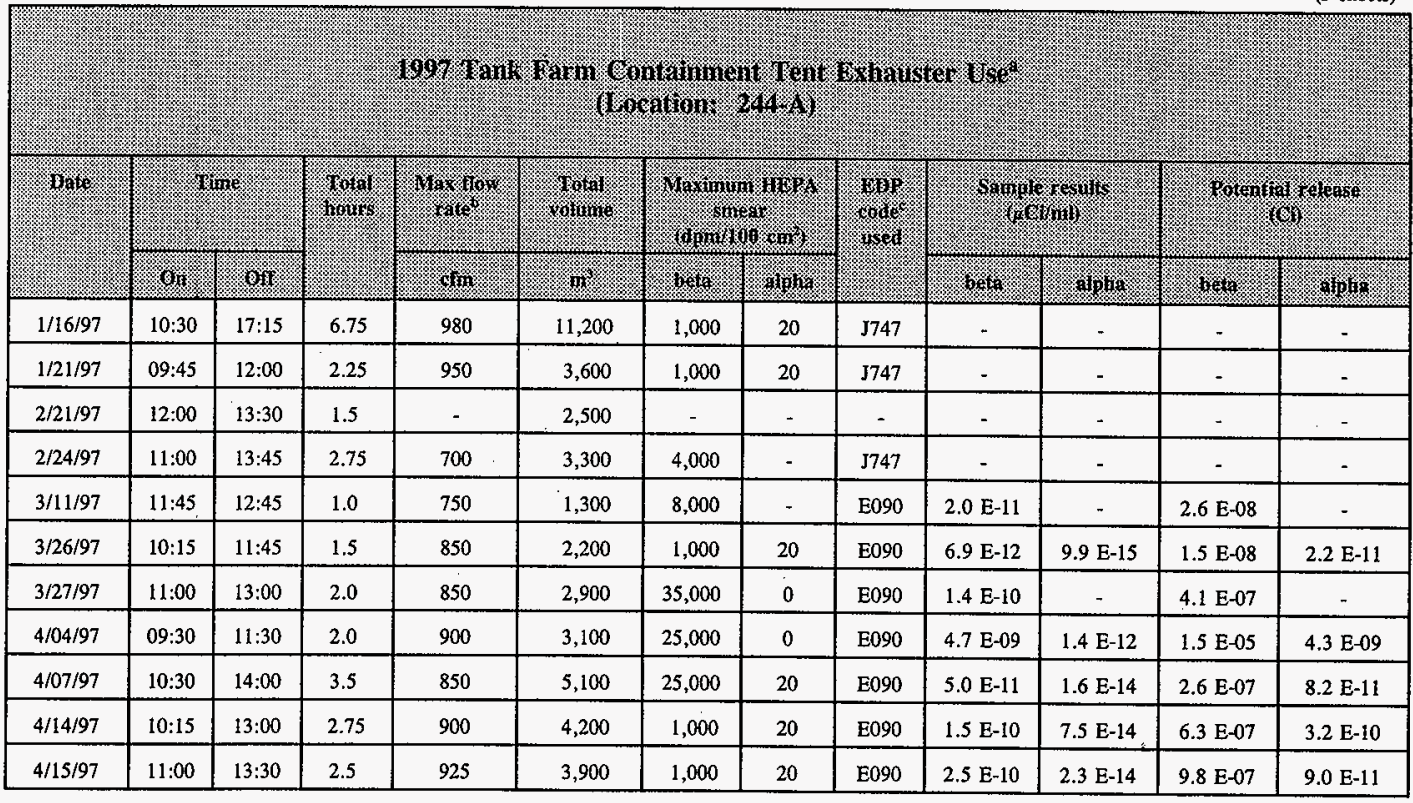


Table 5-3

(3 sheets)

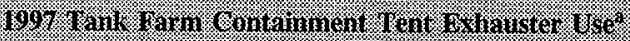
(Eocation: 24.4 .

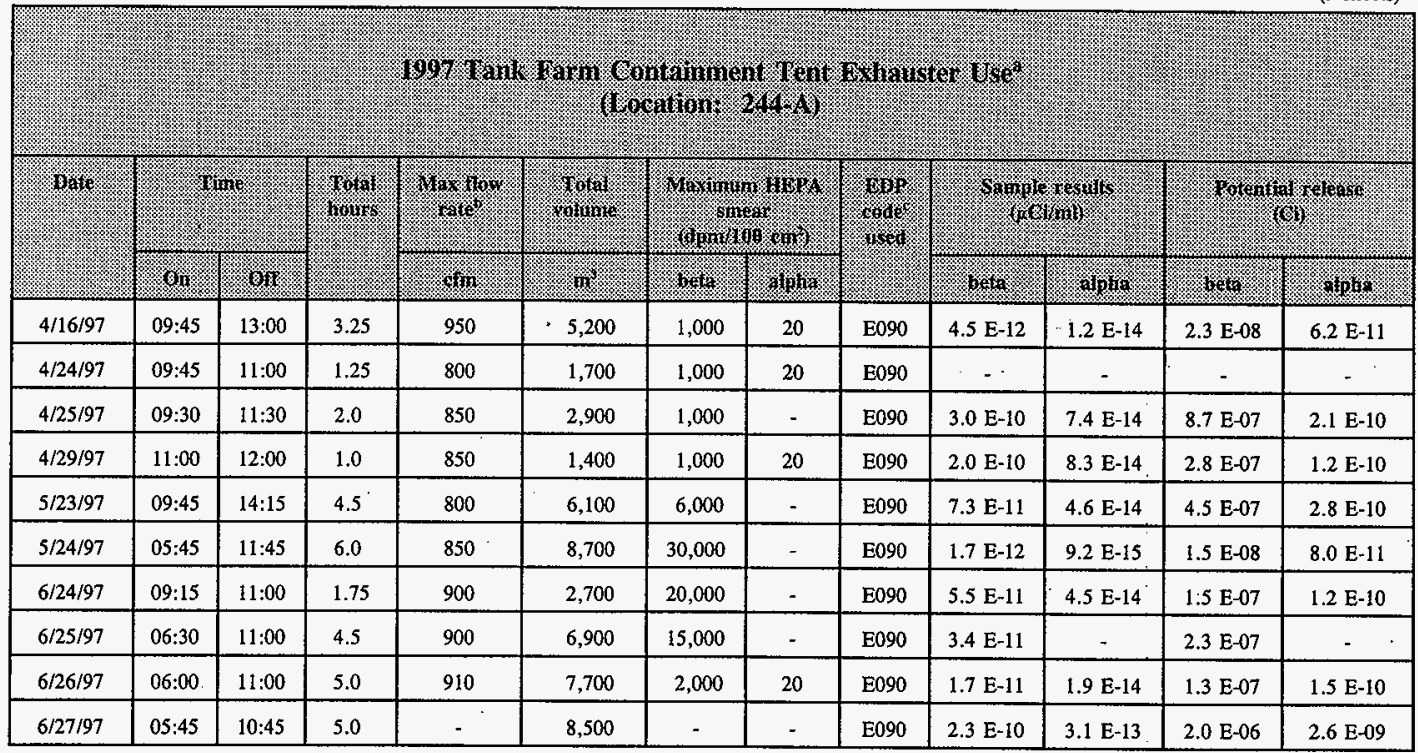


Table 5-3

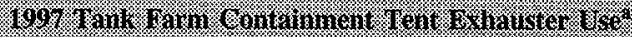

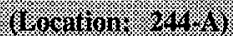

\begin{tabular}{|c|c|c|c|c|c|c|c|c|c|c|c|c|}
\hline 10 & & & 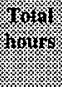 & 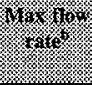 & 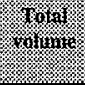 & 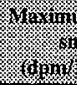 & 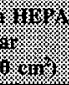 & 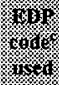 & 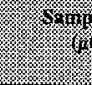 & 3. & \% & \% \\
\hline & 80 & \%) & & 18 & 湆 & toxt & 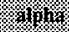 & & 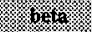 & 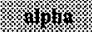 & xes & (2) \\
\hline $6 / 30 / 97$ & $05: 00$ & $13: 30$ & 8.5 & 800 & 11,600 & 2,000 & - & E090 & $8.9 \mathrm{E}-12$ & $2.9 \mathrm{E}-14$ & $1.0 \mathrm{E}-07$ & 3.4 E-10 \\
\hline $7 / 2 / 97$ & $06: 15$ & $10: 45$ & 4.5 & - & 7,600 & - & - & - & $\therefore$ & - & - & - \\
\hline $7 / 3 / 97$ & $07: 00$ & $07: 30$ & 0.5 & 800 & 700 & 1,000 & - & E090 & $1.2 \mathrm{E}-08$ & $5.5 \mathrm{E}-12$ & $8.4 \mathrm{E}-06$ & $3.9 \mathrm{E}-09$ \\
\hline $7 / 8 / 97$ & $09: 45$ & $10: 15$ & 0.5 & 800 & 700 & 6,000 & - & $\mathrm{E} 090$ & $8.8 \mathrm{E}-11$ & $2.2 \mathrm{E}-13$ & $6.2 \mathrm{E}-08$ & $1.5 \mathrm{E}-10$ \\
\hline $7 / 10 / 97$ & $07: 15$ & $11: 45$ & 4.5 & 800 & 6,100 & 8,000 & - & $\mathrm{E} 090$ & $2.5 \mathrm{E}-10$ & $1.8 \mathrm{E}-13$ & $1.5 \mathrm{E}-06$ & $1.1 \mathrm{E}-09$ \\
\hline $7 / 14 / 97$ & $05: 45$ & $07: 00$ & 1.25 & - & 1,900 & 20,000 & - & $\mathrm{E} 090$ & $4.7 \mathrm{E}-11$ & $5.5 \mathrm{E}-14$ & $8.9 \mathrm{E}-08$ & $1.0 \mathrm{E}-10$ \\
\hline \multicolumn{5}{|c|}{ Total volume exhausted } & 136,100 & \multicolumn{3}{|c|}{ Max concentration } & $1.2 \mathrm{E}-08$ & $5.5 \mathrm{E}-12$ & & \\
\hline \multicolumn{11}{|c|}{ POTENTIAL RELEASE USING AIR SAMPL̀E DATA } & $3.2 \mathrm{E}-05$ & $1.4 \mathrm{E}-08$ \\
\hline
\end{tabular}

a $1 \mathrm{cfm}=28.32 \mathrm{Lpm} ; 1 \mathrm{dpm} / 100 \mathrm{~cm}^{2}=60 \mathrm{~Bq} / 100 \mathrm{~cm}^{2} ; 1 \mu \mathrm{Ci} / \mathrm{mL}=3.7 \mathrm{E}+10 \mathrm{~Bq} / \mathrm{m}^{3} ; 1 \mathrm{Ci}=3.7 \mathrm{E}+10 \mathrm{~Bq}$.

b Flow rates marked as "-" were assumed to be $1.0 \mathrm{E}+03 \mathrm{cfm}(2.8 \mathrm{E}+03 \mathrm{Lpm})$

c EDP Code - sampler location code. 


\subsubsection{NOC for the 3732 Process Equipment Development Laboratory Demolition}

The Notice of Construction for the 3732 Process Equipment Development Laboratory Demolition activity requires concentrations from the near-facility ambient air monitoring stations be reported in the annual Radionuclide Air Emissions Report for the Hanford Site. The information provided in Tables 5-4 and 5-5 fulfills this requirement.

Table 5-4

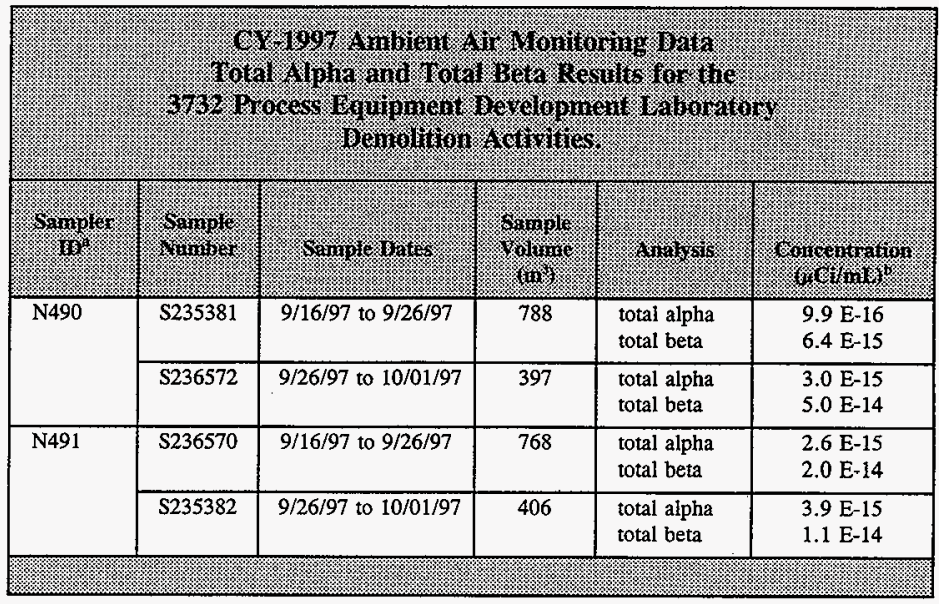

Notes:

a ID = Identification, i.e., the alpha-numeric designator for the respective sampler.

b $1 \mu \mathrm{Ci}=3.7 \mathrm{E}+04 \mathrm{bq} ; 1 \mathrm{~mL}=1.0 \mathrm{E}-06 \mathrm{~m}^{3}$. 
Table 5-5

\begin{tabular}{|c|c|c|c|c|c|}
\hline 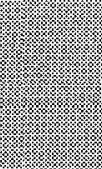 & 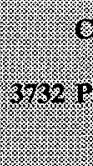 & 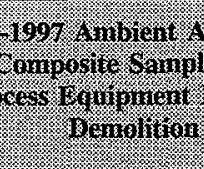 & 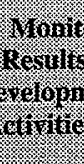 & 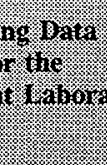 & : \\
\hline 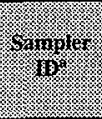 & sainivicin & in & 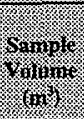 & 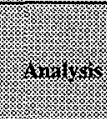 & 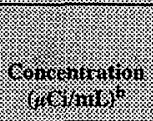 \\
\hline $\mathrm{N} 490$ & S246884 & $9 / 16 / 97$ to $10 / 01 / 97$ & 1185 & $\begin{array}{l}{ }^{60} \mathrm{Co} \\
{ }^{65} \mathrm{Zn} \\
{ }^{103} \mathrm{Ru} \\
{ }^{106} \mathrm{Ru} \\
{ }^{113} \mathrm{Sn} \\
{ }^{125} \mathrm{Sb} \\
{ }^{134} \mathrm{Cs} \\
{ }^{137} \mathrm{Cs} \\
{ }^{144} \mathrm{CePr} \\
{ }^{152} \mathrm{Eu} \\
{ }^{154} \mathrm{Eu} \\
{ }^{155} \mathrm{Eu}\end{array}$ & $\begin{array}{l}\text { ND } \\
\text { ND } \\
\text { ND } \\
\text { ND } \\
\text { ND } \\
\text { ND } \\
\text { ND } \\
\text { ND } \\
\text { ND } \\
\text { ND } \\
\text { ND } \\
\text { ND }\end{array}$ \\
\hline N491 & \$246885 & $9 / 16 / 97$ to $10 / 01 / 97$ & 1175 & $\begin{array}{l}{ }^{60} \mathrm{Co} \\
{ }^{65} \mathrm{Zn} \\
{ }^{103} \mathrm{Ru} \\
{ }^{106} \mathrm{Ru} \\
{ }^{113} \mathrm{Sn} \\
{ }^{125} \mathrm{Sb} \\
{ }^{134} \mathrm{Cs} \\
{ }^{137} \mathrm{Cs} \\
{ }^{144} \mathrm{CePr} \\
{ }^{152} \mathrm{Eu} \\
{ }^{154} \mathrm{Eu} \\
{ }^{155} \mathrm{Eu}\end{array}$ & $\begin{array}{l}\text { ND } \\
\text { ND } \\
\text { ND } \\
\text { ND } \\
\text { ND } \\
\text { ND } \\
\text { ND } \\
\text { ND } \\
\text { ND } \\
\text { ND } \\
\text { ND } \\
\text { ND }\end{array}$ \\
\hline
\end{tabular}

\section{Notes:}

a ID = Identification, i.e., the alpha-numeric designator for the respective sampler.

b $1 \mu \mathrm{Ci}=3.7 \mathrm{E}+04 \mathrm{bq} ; 1 \mathrm{~mL}=1.0 \mathrm{E}-06 \mathrm{~m}^{3}$.

\subsubsection{NOC for the Excavation and Backfilling Activities in Support of a Potable Water Line Repair}

The Notice of Construction for the Excavation and Backfilling Activities in Support of a Potable Water Line Repair requires that a summary of the radiological survey data be reported in the annual Radionuclide Air Emissions Report for the Hanford Site. Speck beta and gamma contamination was found on broken asphalt and concrete and on one piece of old 
water pipe. The greatest field reading was $60,000 \mathrm{dpm}$. No alpha contamination was detected. No contamination was detected in the soil. The contamination specks were packaged and are awaiting final disposal.

\subsubsection{6-Z-10 Stack Emissions}

The estimated emissions for the 296-Z-10 stack are provided in Table 5-6. Record sampling for this stack was discontinued during June of 1987, because emissions were frequently below detection limits and because the potential for exceeding $4 \%$ of the Derived Concentration Guides (DCGs) was minimal (refer to DOE Order 5400.5 and WHC 1988). During 1997 , this stack operated intermittently to supply building ventilation to the office areas of the 231- $\mathrm{Z}$ Building. The emissions estimate provided in Table 5-6 is considered to be a conservative estimate, based on historical emissions from this point source.

Table 5-6

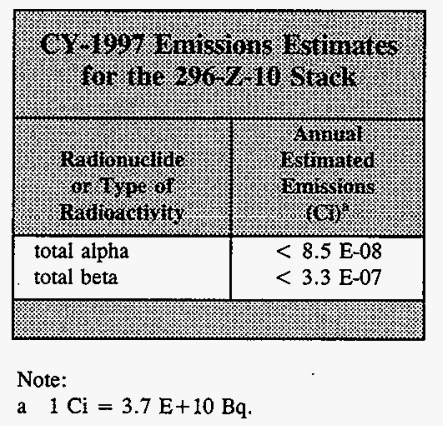

\subsection{QUALITY ASSURANCE PROGRAMS' COMPLIANCE STATUS}

The document Quality Assurance Program for Radionuclide Airborne Emissions Monitoring (WHC 1995b) contain appendices that provide point-by-point comparisons of the major point source monitoring systems to the quality assurance criteria of 40 CFR 61 , Appendix B Method 114. The document Effluent Monitoring Quality Assurance Project Plan for Radioactive Airborne Emissions Data (WHC 1995a) contains the quality assurance activities associated with data collection and reporting. FDH and BHI airborne effluent monitoring activities are conducted in accordance with federal and state regulations as well as the documents listed below. 
PNNL has implemented a quality assurance plan for facility effluent monitoring, as required by 40 CFR 61 , Appendix B, Method 114 . The effluent monitoring quality assurance plan incorporates guidance from the documents listed in the paragraph below.

The quality assurance programs described by these plans are based on the following documentation:

- EPA QAMS-005/80, Interim Guidelines for Preparing Quality Assurance Project Plans, (EPA 1983)

- DOE 5700.6C, Quality Assurance

- DOE 5400.1, General Environmental Protection Program

- DOE/EH-0173T, Environmental Regulatory Guide for Radiological Effluent Monitoring and Environmental Surveillance, (DOE 1991)

- applicable criteria of ASME NQA-1, Quality Assurance Requirements for Nuclear Facilities, (ASME 1989). 


\subsection{REFERENCES}

ASME, 1989, Quality Assurance Program for Nuclear Facilities, American Society for Testing and Material, NQA-1-1989 Edition, New York.

Brown, R. M., G. L. Ogram, and F. S. Spencer, 1990, Oxidation and Dispersion of HT in the Environment: The August 1986 Field Experiment at Chalk River, Health Physics 58(2):171-181.

DOE, 1991, Environmental Regulatory Guide for Radiological Effluent Monitoring and Environmental Surveillance, DOE/EH-0173T, U.S. Department of Energy, Washington D.C.

DOE-RL, 1988, Hanford Site Dangerous Waste Part A Permit Application, DOE/RL-88-21, U.S. Department of Energy, Richland Operations Office, Richland, Washington.

DOE-RL, 1991, Calendar Year 1990 Air Emissions Report for the Hanford Site, DOE/RL-91-10, U.S. Department of Energy, Richland Field Office, Richland, Washington.

DOE-RL, 1992, Radionuclide Air Emissions Report for the Hanford Site, Calendar Year 1991, DOE/RL-92-30, U.S. Department of Energy, Richland Field Office, Richland, Washington.

DOE-RL, 1993, Radionuclide Air Emissions Report for the Hanford Site, Calendar Year 1992, DOE/RL-93-36, U.S. Department of Energy, Richland Field Office, Richland, Washington.

DOE-RL, 1994, Radionuclide Air Emissions Report for the Hanford Site, Calendar Year 1993, DOE/RL-94-51, U.S. Department of Energy, Richland Field Office, Richland, Washington.

DOE-RL, 1995, Radionuclide Air Emissions Report for the Hanford Site, Calendar Year 1994, DOE/RL-95-49, U.S. Department of Energy, Richland Field Office, Richland, Washington.

DOE-RL, 1996, Radionuclide Air Emissions Report for the Hanford Site, Calendar Year 1995, DOE/RL-96-37, U.S. Department of Energy, Richland Field Office, Richland, Washington.

DOE-RL, 1997a, Radionuclide Air Emissions Report for the Hanford Site, Calendar Year 1996, DOE/RL-97-43, U.S. Department of Energy, Richland Field Office, Richland, Washington. 
DOE-RL, 1997b, Radioactive Air Emissions Notice of Construction Portable/Temporary Air Emission Units, DOE/RL-96-75 Rev. 1, U.S. Department of Energy, Richland Field Office, Richland, Washington.

EPA, 1980, Interim Guidelines and Specifications for Preparing Quality Assurance Project Plans, EPA/500/80, U.S. Environmental Protection Agency, Washington, D.C.

EPA, 1992, User's Guide for CAP88-PC, Version 1.0, 402-B-92-001, Office of Radiation Programs, U.S. Environmental Protection Agency, Las Vegas, Nevada.

PNL, 1988, GENII - The Hanford Environmental Radiation Dosimetry Software System, PNL-6584, Vols. 1-3, Pacific Northwest Laboratory, Richland, Washington.

PNL, 1990, Hanford Site Environmental Report for Calendar Year 1989, PNL-7346, Pacific Northwest Laboratory, Richland, Washington.

PNL, 1991, Hanford Area 1990 Population and 50-year Projections, PNL-7.803, Pacific Northwest Laboratory, Richland, Washington.

PNL, 1993, Recommended Environmental Dose Calculation Methods and Hanford-Specific Parameters, PNL-3777, Rev. 2., Pacific Northwest Laboratory, Richland, Washington.

PNL, 1997, Hanford Site National Environmental Policy Act (NEPA) Characterization, PNL-6415 Rev. 9, Pacific Northwest Laboratory, Richland, Washington.

PNNL, 1998a, Hanford Site Environmental Report for Calendar Year 1997, PNNL-11795, Pacific Northwest National Laboratory, Richland, Washington.

PNNL, 1998b, 1997 Surface Environmental Surveillance Data, PNNL-11796, Pacific Northwest National Laboratory, Richland, Washington.

PNNL, 1998c, Hanford Site Climatological Data Summary 1997, with Historical Data, PNNL-11794, Pacific Northwest Laboratory, Richland, Washington.

SNL, 1992, User's Guide for GENIL-S: A Code for Statistical and Deterministic Simulations of Radiation Doses to Humans from Radionuclides in the Environment, SAND91-0561A, Sandia National Laboratories, Albuquerque, New Mexico.

WHC, 1988, Westinghouse Hanford Company Effluent Releases and Solid Waste Management Report for 1987: 200/600/1100 Areas, WHC-EP-0141, Westinghouse Hanford Company, Richland, Washington.

WHC, 1995a, Effluent Monitoring Quality Assurance Project Plan for Radioactive Airborne Emissions Data, WHC-EP-0528-2, Westinghouse Hanford Company, Richland, Washington. 
WHC, 1995b, Quality Assurance Program Plan for Radionuclide Airborne Emissions Monitoring, WHC-EP-0536-3, Westinghouse Hanford Company, Richland, Washington.

WMNW, 1998, Hanford Site Near-Facility Environmental Monitoring Annual Report, Calendar Year 1997, HNF-EP-0573-6, Waste Management Federal Services, Inc. Northwest Operations, Richland, Washington.

Zacks, R. S., March 1995, Biological Diversity. Inventory and Analysis at the Hanford Site: Insects, Department of Entomology, Washington State University, Pullman, Washington. 
DOE/RL-98-33

This page intentionally left blank. 
DOE/RL-98-33

\section{APPENDIX}

DOSE MODELING AND METEOROLOGICAL DATA 
DOE/RL-98-33

This page intentionally left blank. 
Figure A-1. Meteorological Station Map and Wind Roses for Calendar Year 1997.

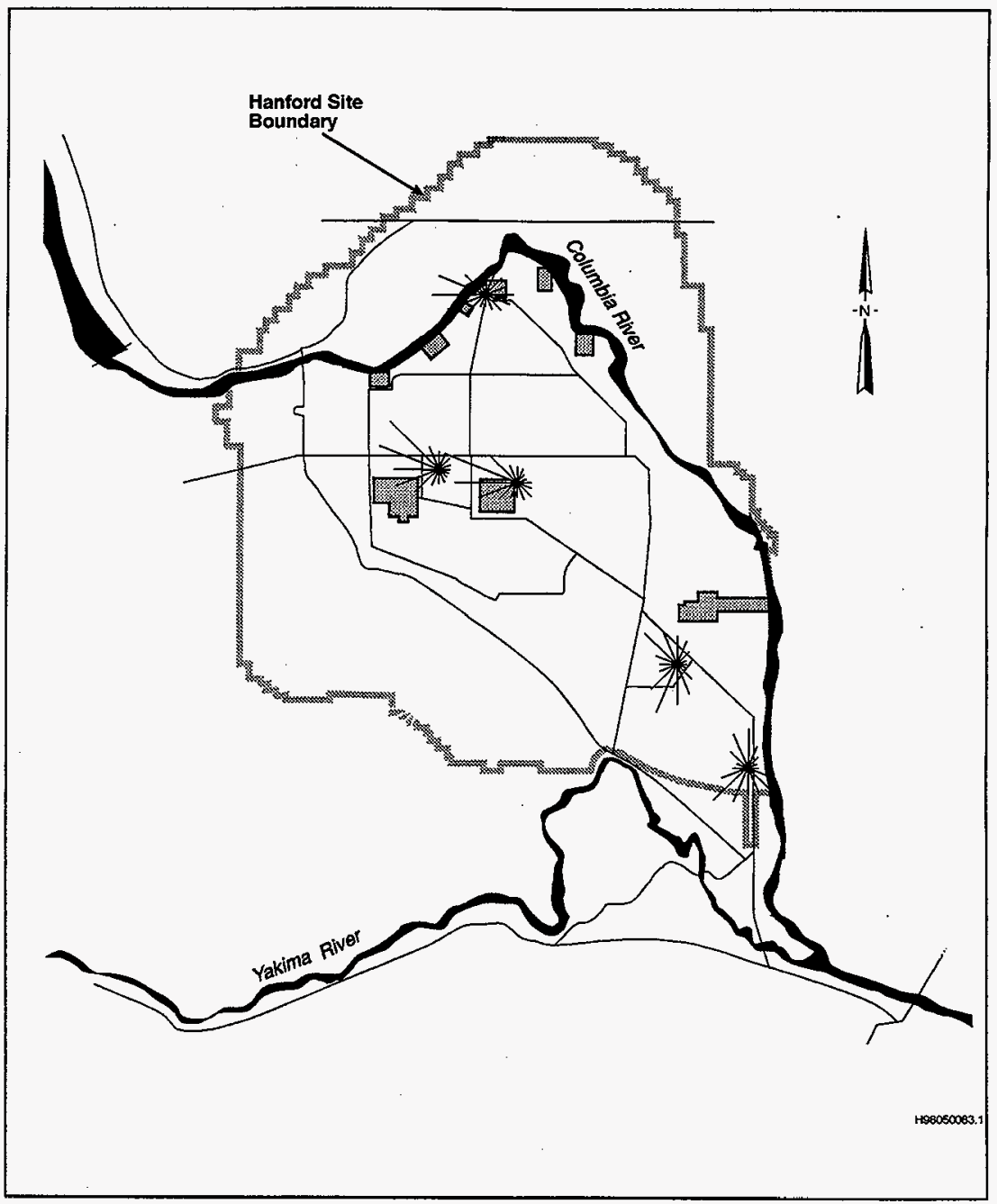


Figure A-2. 100 Area Wind Rose \& Histogram.

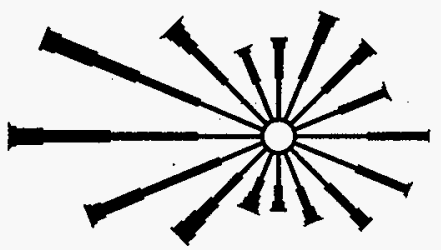

(a) Wind Rose

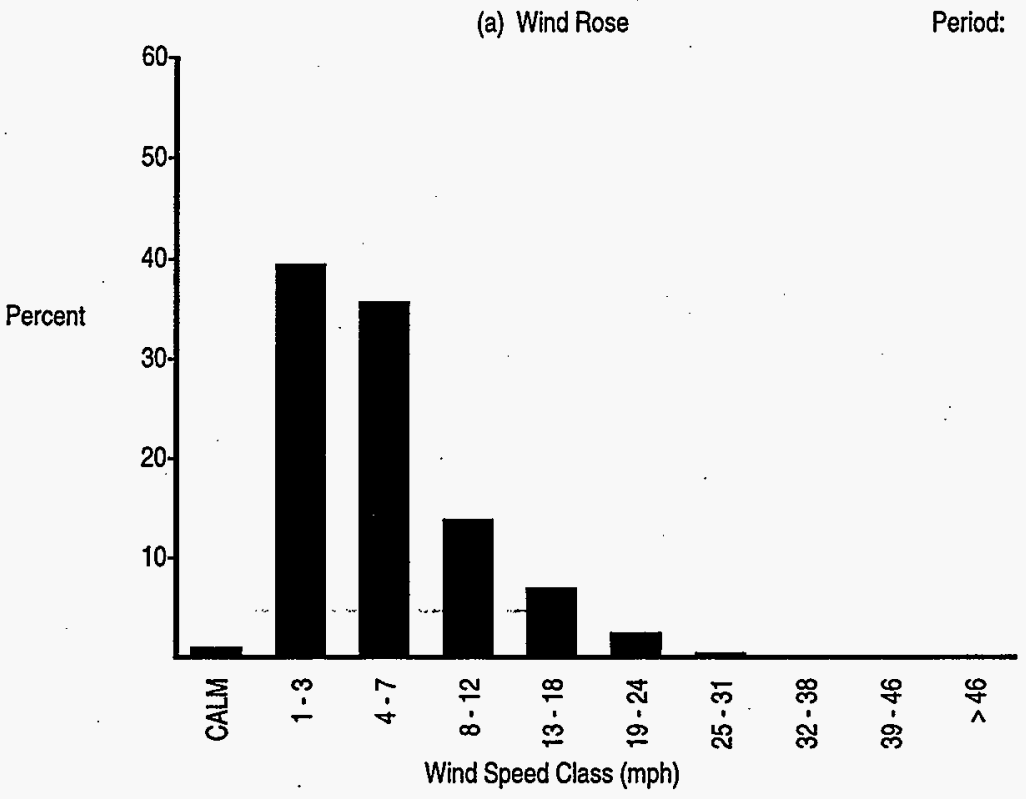

(b) Wind Speed Histogram

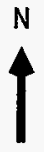

Station \#13 - 100N 
Figure A-3. 200 East Area Wind Rose \& Histogram.
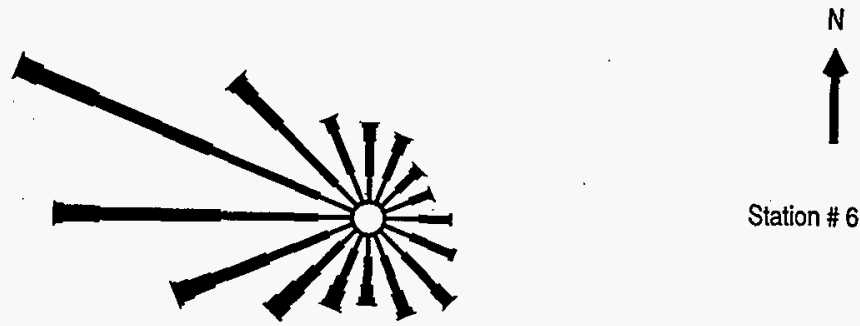

Station \# 6 - 200E

(a) Wind Rose

Period: $1 / 97-12 / 97$

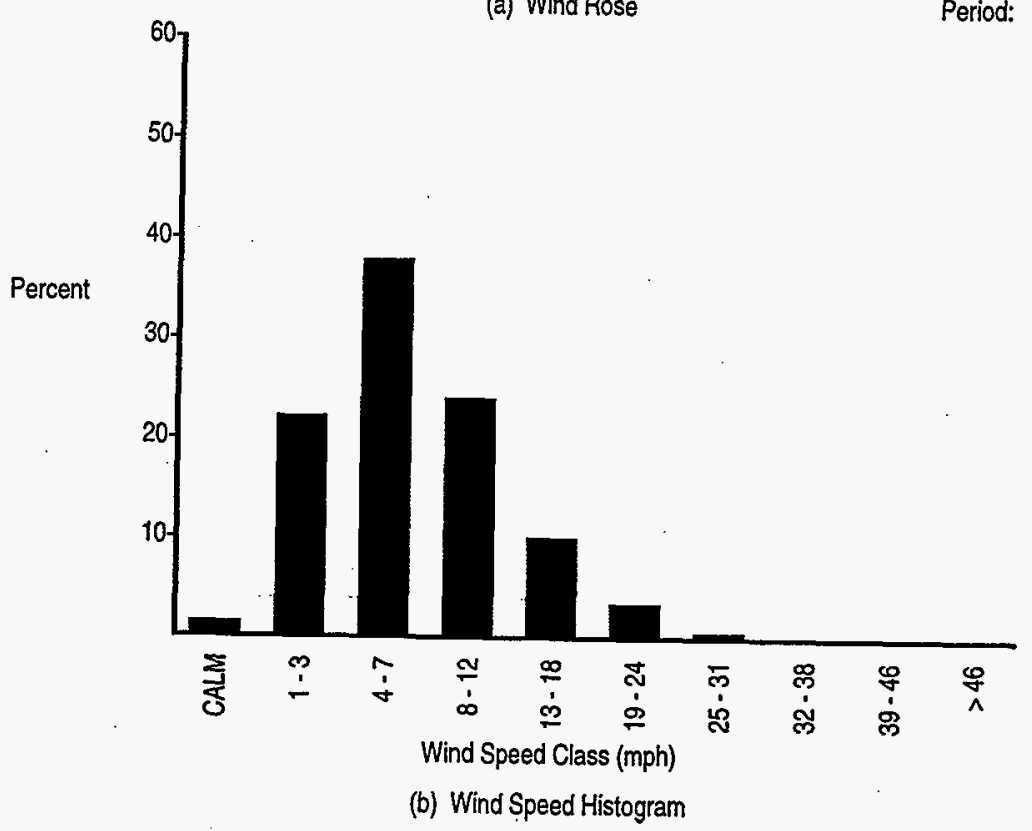


Figure A-4. 200 West Area Wind Rose \& Histogram.
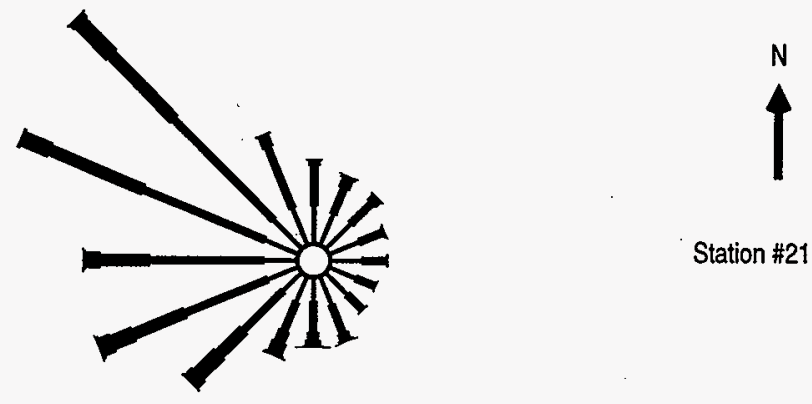

Station \#21- HMS

(a) Wind Rose

Period: $1 / 97-12 / 97$

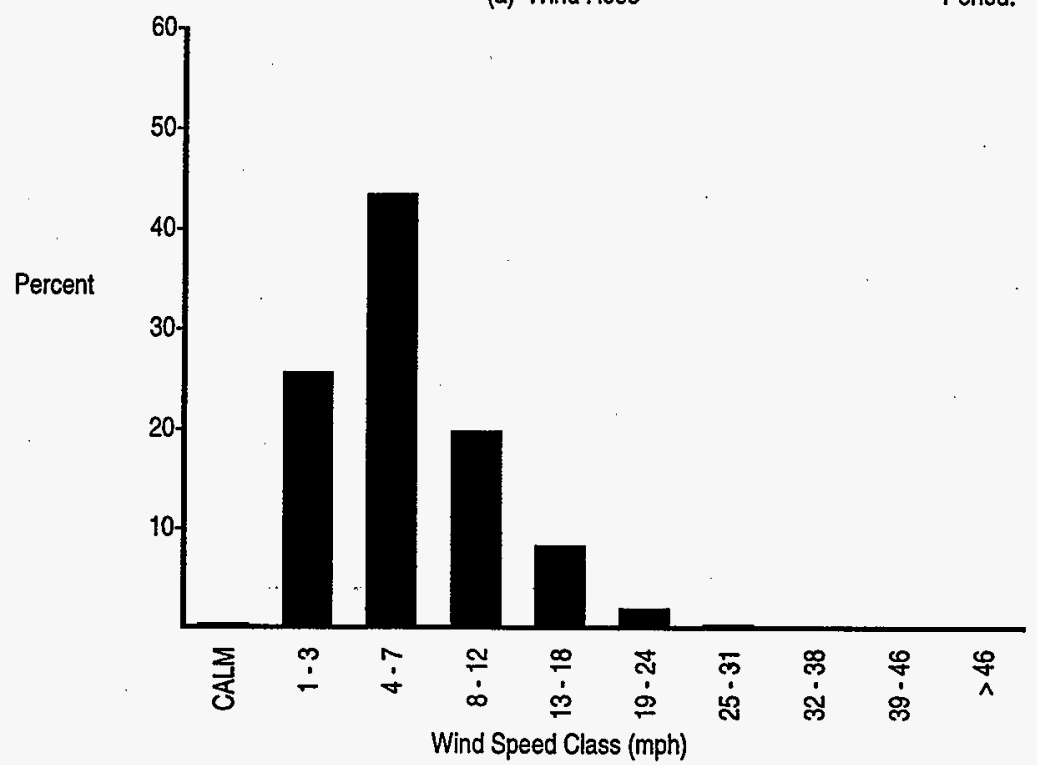

(b) Wind Speed Histogram 
Figure A-5. 300 Area Wind Rose \& Histogram.

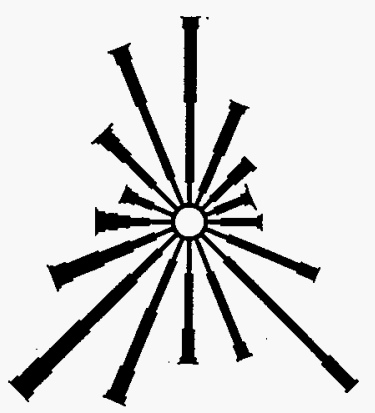

(a) Wind Rose

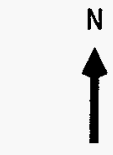

Station \#11 - 300A

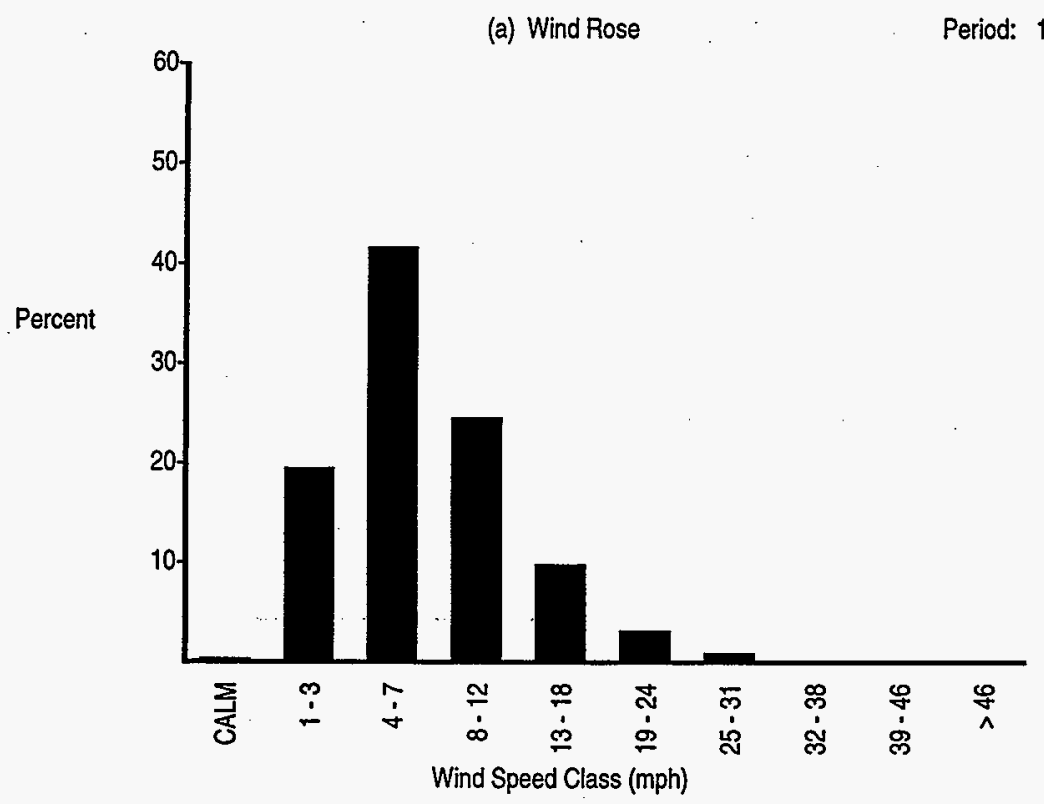

(b) Wind Speed Histogram 
Figure A-6. 400 Area Wind Rose \& Histogram.

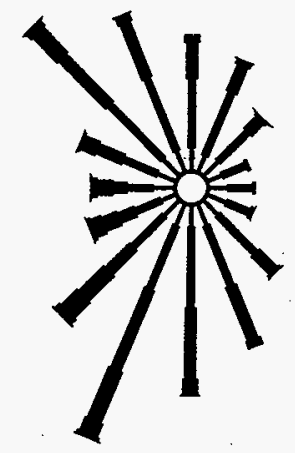

(a) Wind Rose

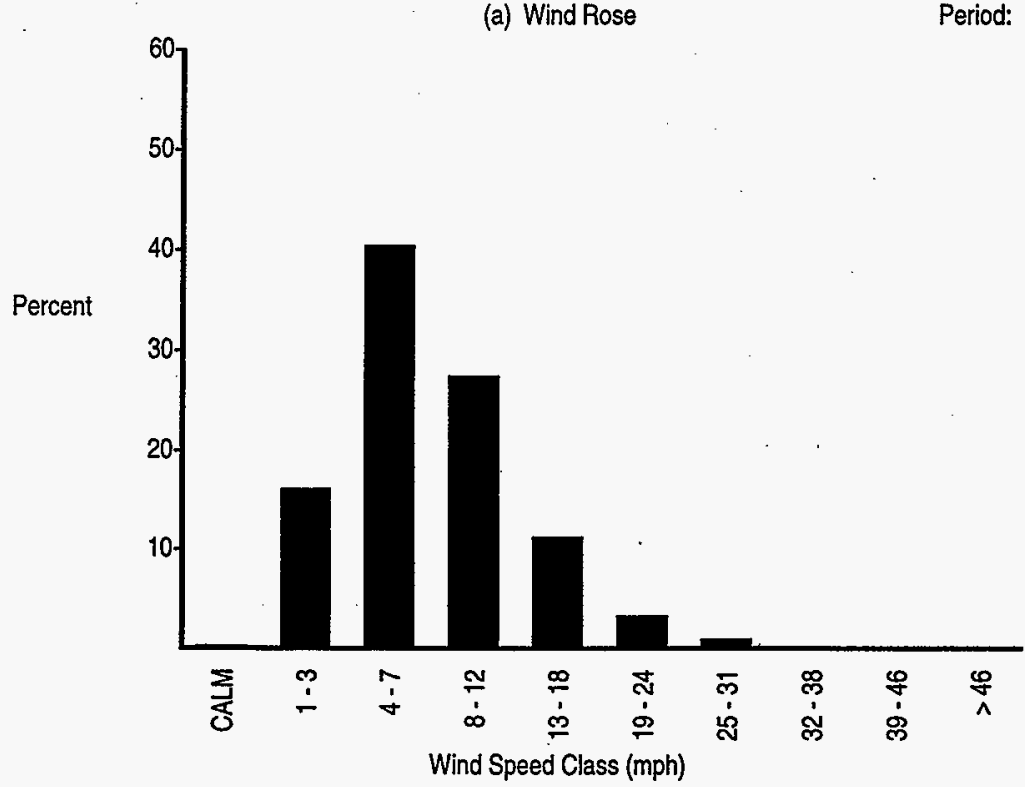

(b) Wind Speed Histogram
Station \# 9 - FFTF

Period: 1/97 - 12/97 
Table A-1. Annual Average Dispersion Factor Around the 100 Area During 1997 for a 10 -Meter Release Height.

\begin{tabular}{|c|c|c|c|c|c|c|c|c|c|c|}
\hline st: & $8 \mathrm{~km}$ & $4 \mathrm{~km}$ & $.0 \mathrm{~km}$ & $.6 \mathrm{~km}$ & $7.2 \mathrm{~km}$ & $2 \mathrm{~km}$ & $4 \mathrm{~km}$ & $40 \mathrm{~km}$ & $56 \mathrm{~km}$ & $72 \mathrm{~km}$ \\
\hline \multicolumn{11}{|l|}{ ir: } \\
\hline IE & $\begin{array}{l}2.21 E-06 \\
2.14 E-06 \\
3.16 E-06 \\
4.89 E-06\end{array}$ & $\begin{array}{l}-07 \\
-07 \\
-07 \\
-06\end{array}$ & $\begin{array}{l}2.0 \\
2.9\end{array}$ & $\begin{array}{l}1.24 \\
1.82\end{array}$ & $\begin{array}{l}8.6 \\
1.2\end{array}$ & $\begin{array}{l}-08 \\
-08 \\
-08 \\
-07\end{array}$ & $\begin{array}{l}08 \\
08 \\
08 \\
08\end{array}$ & $\begin{array}{l}19 \\
9 \\
18 \\
8\end{array}$ & $\begin{array}{l}E-09 \\
E-09 \\
E-09 \\
E-08\end{array}$ & \\
\hline & $\begin{array}{l}5.89 \mathrm{E}-06 \\
4.17 \mathrm{E}-06 \\
2.62 \mathrm{E}-06\end{array}$ & $\begin{array}{l}1.21 \mathrm{E}-06 \\
8.45 \mathrm{E}-07 \\
5.18 \mathrm{E}-07\end{array}$ & $\begin{array}{l}5.76 \\
4.00 \\
2.45\end{array}$ & $\begin{array}{l}3.5 \\
2.4 \\
1.5\end{array}$ & $\begin{array}{l}2.4 \\
1.7 \\
1.0\end{array}$ & $\begin{array}{l}1.2 \\
8.4 \\
5.1\end{array}$ & & $\begin{array}{l}2 . \\
1 . \\
1 .\end{array}$ & $\begin{array}{l}1.5 \\
1.0 \\
6.5\end{array}$ & $\begin{array}{l}1.1 \\
7.7 \\
4.7\end{array}$ \\
\hline & $\begin{array}{l}2.63 \mathrm{E}-06 \\
3.18 \mathrm{E}-06 \\
3.48 \mathrm{E}-06 \\
4.07 \mathrm{E}-06\end{array}$ & $\begin{array}{l}5.20 \mathrm{E}-07 \\
6.34 \mathrm{E}-07 \\
6.90 \mathrm{E}-07 \\
8.68 \mathrm{E}-07\end{array}$ & $\begin{array}{l}3.00 \\
3.26 \\
4.16\end{array}$ & $\begin{array}{l}1.51 E-07 \\
1.85 E-07 \\
2.01 E-07 \\
2.59 E-07\end{array}$ & $\begin{array}{l}1.06 \mathrm{E}-07 \\
1.29 \mathrm{E}-07 \\
1.41 \mathrm{E}-07 \\
1.82 \mathrm{E}-07\end{array}$ & $\begin{array}{l}5.1 \\
6.3 \\
6.8 \\
9.0\end{array}$ & & $\begin{array}{l}1.2 \\
1.2 \\
1.7\end{array}$ & & \\
\hline$W$ & $\begin{array}{l}5.62 \mathrm{E}-06 \\
5.06 \mathrm{E}-06 \\
3.93 \mathrm{E}-06 \\
2.73 \mathrm{E}-06\end{array}$ & $\begin{array}{l}1.17 \mathrm{E}-06 \\
1.02 \mathrm{E}-06 \\
7.81 \mathrm{E}-07 \\
5.44 \mathrm{E}-07\end{array}$ & $\begin{array}{l}5.57 E-07 \\
4.80 E-07 \\
3.68 E-07 \\
2.57 E-07\end{array}$ & $\begin{array}{l}3.44 \mathrm{E}-07 \\
2.96 \mathrm{E}-07 \\
2.26 \mathrm{E}-07 \\
1.58 \mathrm{E}-07\end{array}$ & $\begin{array}{l}2.42 \mathrm{E}-07 \\
2.07 \mathrm{E}-07 \\
1.58 \mathrm{E}-07 \\
1.10 \mathrm{E}-07\end{array}$ & $\begin{array}{l}1.19 \mathrm{E}-07 \\
1.01 \mathrm{E}-07 \\
7.71 \mathrm{E}-08 \\
5.38 \mathrm{E}-08\end{array}$ & $\begin{array}{l}3.91 \mathrm{E} \\
2.97 \mathrm{E} \\
2.08 \mathrm{E}\end{array}$ & $\begin{array}{l}2.34 \mathrm{E}-08 \\
1.96 \mathrm{E}-08 \\
1.49 \mathrm{E}-08 \\
1.04 \mathrm{E}-08\end{array}$ & $\begin{array}{l}1.26 \mathrm{E} \\
9.55 \mathrm{E} \\
6.67 \mathrm{E}\end{array}$ & $\begin{array}{l}1.08 \mathrm{E}-08 \\
9.02 \mathrm{E}-09 \\
6.85 \mathrm{E}-09 \\
4.79 \mathrm{E}-09\end{array}$ \\
\hline
\end{tabular}


Table A-2. Annual Average Dispersion Factor Around the 200 Areas During 1997 for an 89-Meter Release Height.

\begin{tabular}{|c|c|c|c|c|c|c|c|c|c|c|}
\hline Dist & $0.8 \mathrm{~km}$ & $2.4 \mathrm{~km}$ & $4.0 \mathrm{~km}$ & $5.6 \mathrm{~km}$ & $7.2 \mathrm{~km}$ & $12 \mathrm{~km}$ & $24 \mathrm{~km}$ & $40 \mathrm{~km}$ & $56 \mathrm{~km}$ & $72 \mathrm{~km}$ \\
\hline \multicolumn{11}{|l|}{ Dir: } \\
\hline VE & $\begin{array}{l}3.42 \mathrm{E}-08 \\
3.56 \mathrm{E}-08 \\
4.29 \mathrm{E}-08 \\
3.48 \mathrm{E}-08\end{array}$ & $\begin{array}{l}2.53 E-08 \\
2.60 E-08 \\
3.48 E-08 \\
3.73 E-08\end{array}$ & $\begin{array}{l}1.99 E-08 \\
2.12 E-08 \\
2.87 E-08 \\
3.26 E-08\end{array}$ & $\begin{array}{l}1.56 \mathrm{E}-08 \\
1.69 \mathrm{E}-08 \\
2.30 \mathrm{E}-08 \\
2.66 \mathrm{E}-08\end{array}$ & $\begin{array}{l}1.26 \mathrm{E}-08 \\
1.39 \mathrm{E}-08 \\
1.88 \mathrm{E}-08 \\
2.21 \mathrm{E}-08\end{array}$ & $\begin{array}{l}7.80 \mathrm{E}-09 \\
8.76 \mathrm{E}-09 \\
1.18 \mathrm{E}-08 \\
1.42 \mathrm{E}-08\end{array}$ & $\begin{array}{l}3.83 E-09 \\
4.40 E-09 \\
5.81 E-09 \\
7.21 E-09\end{array}$ & $\begin{array}{l}2.22 \mathrm{E}-09 \\
2.58 \mathrm{E}-09 \\
3.35 \mathrm{E}-09 \\
4.24 \mathrm{E}-09\end{array}$ & $\begin{array}{l}1.54 E-09 \\
1.80 E-09 \\
2.31 E-09 \\
2.96 E-09\end{array}$ & $\begin{array}{l}1.17 E-09 \\
1.37 E-09 \\
1.74 E-09 \\
2.25 E-09\end{array}$ \\
\hline $\begin{array}{l}\text { SE. } \\
\text { SSE }\end{array}$ & $\begin{array}{l}2.19 E-08 \\
6.95 E-08 \\
1.17 E-07 \\
9.68 E-08\end{array}$ & $\begin{array}{l}3.34 \mathrm{E}-08 \\
7.46 \mathrm{E}-08 \\
9.74 \mathrm{E}-08 \\
6.77 \mathrm{E}-08\end{array}$ & $\begin{array}{l}3.30 \mathrm{E}-08 \\
6.72 \mathrm{E}-08 \\
7.73 \mathrm{E}-08 \\
5.22 \mathrm{E}-08\end{array}$ & $\begin{array}{l}2.88 \mathrm{E}-08 \\
5.58 \mathrm{E}-08 \\
6.06 \mathrm{E}-08 \\
4.01 \mathrm{E}-08\end{array}$ & $\begin{array}{l}2.48 \mathrm{E}-08 \\
4.66 \mathrm{E}-08 \\
4.92 \mathrm{E}-08 \\
3.21 \mathrm{E}-08\end{array}$ & $\begin{array}{l}1.69 E-08 \\
3.01 E-08 \\
3.05 E-08 \\
1.94 E-08\end{array}$ & $\begin{array}{l}9.07 \mathrm{E}-09 \\
1.51 \mathrm{E}-08 \\
1.50 \mathrm{E}-08 \\
9.16 \mathrm{E}-09\end{array}$ & $\begin{array}{l}5.49 E-09 \\
8.80 E-09 \\
8.60 E-09 \\
5.15 E-09\end{array}$ & $\begin{array}{l}3.89 \mathrm{E}-09 \\
6.09 \mathrm{E}-09 \\
5.93 \mathrm{E}-09 \\
3.51 \mathrm{E}-09\end{array}$ & $\begin{array}{l}2.99 E-09 \\
4.61 E-09 \\
4.47 E-09 \\
2.62 E-09\end{array}$ \\
\hline $\begin{array}{l}\text { S } \\
\text { SSW } \\
\text { SW } \\
\text { WSW }\end{array}$ & $\begin{array}{l}5.75 E-08 \\
8.70 E-08 \\
8.02 E-08 \\
7.72 E-08\end{array}$ & $\begin{array}{l}3.41 E-08 \\
4.73 E-08 \\
4.24 E-08 \\
4.67 E-08\end{array}$ & $\begin{array}{l}2.58 \mathrm{E}-08 \\
3.49 \mathrm{E}-08 \\
3.02 \mathrm{E}-08 \\
3.40 \mathrm{E}-08\end{array}$ & $\begin{array}{l}1.99 \mathrm{E}-08 \\
2.64 \mathrm{E}-08 \\
2.26 \mathrm{E}-08 \\
2.54 \mathrm{E}-08\end{array}$ & $\begin{array}{l}1.59 E-08 \\
2.08 E-08 \\
1.79 E-08 \\
2.00 E-08\end{array}$ & $\begin{array}{l}9.73 \mathrm{E}-09 \\
1.22 \mathrm{E}-08 \\
1.07 \mathrm{E}-08 \\
1.17 \mathrm{E}-08\end{array}$ & $\begin{array}{l}4.68 \mathrm{E}-09 \\
5.64 \mathrm{E}-09 \\
5.05 \mathrm{E}-09 \\
5.39 \mathrm{E}-09\end{array}$ & $\begin{array}{l}2.66 \mathrm{E}-09 \\
3.13 \mathrm{E}-09 \\
2.85 \mathrm{E}-09 \\
2.99 \mathrm{E}-09\end{array}$ & $\begin{array}{l}1.82 \mathrm{E}-09 \\
2.12 \mathrm{E}-09 \\
1.95 \mathrm{E}-09 \\
2.02 \mathrm{E}-09\end{array}$ & $\begin{array}{l}1.37 \mathrm{E}-09 \\
1.58 \mathrm{E}-09 \\
1.46 \mathrm{E}-09 \\
1.50 \mathrm{E}-09\end{array}$ \\
\hline $\begin{array}{l}W \\
W N W \\
\text { NW }\end{array}$ & $\begin{array}{l}6.30 \mathrm{E}-08 \\
4.77 \mathrm{E}-09 \\
6.59 \mathrm{E}-08 \\
3.92 \mathrm{E}-08\end{array}$ & $\begin{array}{l}4.18 \mathrm{E}-08 \\
9.24 \mathrm{E}-09 \\
5.42 \mathrm{E}-08 \\
3.27 \mathrm{E}-08\end{array}$ & $\begin{array}{l}3.29 \mathrm{E}-08 \\
8.36 \mathrm{E}-09 \\
4.37 \mathrm{E}-08 \\
2.68 \mathrm{E}-08\end{array}$ & $\begin{array}{l}2.53 E-08 \\
6.74 E-09 \\
3.45 E-08 \\
2.15 E-08\end{array}$ & $\begin{array}{l}2.02 \mathrm{E}-08 \\
5.47 \mathrm{E}-09 \\
2.80 \mathrm{E}-08 \\
1.78 \mathrm{E}-08\end{array}$ & $\begin{array}{l}1.20 \mathrm{E}-08 \\
3.32 \mathrm{E}-09 \\
1.73 \mathrm{E}-08 \\
1.14 \mathrm{E}-08\end{array}$ & $\begin{array}{l}5.55 \mathrm{E}-09 \\
1.55 \mathrm{E}-09 \\
8.34 \mathrm{E}-09 \\
5.75 \mathrm{E}-09\end{array}$ & $\begin{array}{l}3.07 E-09 \\
8.64 E-10 \\
4.72 E-09 \\
3.37 E-09\end{array}$ & $\begin{array}{l}2.07 \mathrm{E}-09 \\
5.87 \mathrm{E}-10 \\
3.22 \mathrm{E}-09 \\
2.35 \mathrm{E}-09\end{array}$ & $\begin{array}{l}1.54 \mathrm{E}-09 \\
4.38 \mathrm{E}-10 \\
2.41 \mathrm{E}-09 \\
1.78 \mathrm{E}-09\end{array}$ \\
\hline
\end{tabular}


Table A-3. Annual Average Dispersion Factor Around the 300 Area During 1997 for a 10-Meter Release Height.

Dist: $\quad 0.8 \mathrm{~km} \quad 2.4 \mathrm{~km} \quad 4.0 \mathrm{~km} \quad 5.6 \mathrm{~km} \quad 1.2 \mathrm{~km} \quad 12 \mathrm{~km} \quad 24 \mathrm{~km} \quad 40 \mathrm{~km} \quad \underline{5 \mathrm{~km}} \quad \underline{72 \mathrm{~km}}$ Dir:

\begin{tabular}{|c|c|c|c|c|c|c|c|c|c|c|}
\hline $\begin{array}{l}\text { NNE } \\
\text { NE } \\
\text { FNE }\end{array}$ & $\begin{array}{l}3.78 E-06 \\
3.20 E-06 \\
3.12 E-06 \\
2.21 E-06\end{array}$ & $\begin{array}{l}7.75 E-07 \\
6.21 E-07 \\
5.98 E-07 \\
4.33 E-07\end{array}$ & $\begin{array}{l}3.68 \mathrm{E}-07 \\
2.92 \mathrm{E}-07 \\
2.80 \mathrm{E}-07 \\
2.04 \mathrm{E}-07\end{array}$ & $\begin{array}{l}2.28 E-07 \\
1.79 E-07 \\
1.72 E-07 \\
1.25 E-07\end{array}$ & $\begin{array}{l}1.60 E-07 \\
1.25 E-07 \\
1.20 E-07 \\
8.72 E-08\end{array}$ & $\begin{array}{l}7.85 E-08 \\
6.10 E-08 \\
5.86 E-08 \\
4.25 E-08\end{array}$ & $\begin{array}{l}3.05 \mathrm{E}-08 \\
2.36 \mathrm{E}-08 \\
2.27 \mathrm{E}-08 \\
1.64 \mathrm{E}-08\end{array}$ & $\begin{array}{l}1.54 \mathrm{E}-08 \\
1.19 \mathrm{E}-08 \\
1.14 \mathrm{E}-08 \\
8.19 \mathrm{E}-09\end{array}$ & $\begin{array}{l}9.88 \mathrm{E}-09 \\
7.59 \mathrm{E}-09 \\
7.30 \mathrm{E}-09 \\
5.23 \mathrm{E}-09\end{array}$ & $\begin{array}{l}7.11 \mathrm{E}-09 \\
5.45 \mathrm{E}-09 \\
5.24 \mathrm{E}-09 \\
3.75 \mathrm{E}-09\end{array}$ \\
\hline & $\begin{array}{l}1.99 E-06 \\
2.03 E-06 \\
3.37 E-06 \\
4.16 E-06\end{array}$ & $\begin{array}{l}3.93 \mathrm{E}-07 \\
4.28 \mathrm{E}-07 \\
7.05 \mathrm{E}-07 \\
8.66 \mathrm{E}-07\end{array}$ & $\begin{array}{l}1.85 E-07 \\
2.04 E-07 \\
3.36 E-07 \\
4.12 E-07\end{array}$ & $\begin{array}{l}1.14 \mathrm{E}-07 \\
1.26 \mathrm{E}-07 \\
2.08 \mathrm{E}-07 \\
2.54 \mathrm{E}-07\end{array}$ & $\begin{array}{l}7.94 \mathrm{E}-08 \\
8.85 \mathrm{E}-08 \\
1.46 \mathrm{E}-07 \\
1.78 \mathrm{E}-07\end{array}$ & $\begin{array}{l}3.87 \mathrm{E}-08 \\
4.34 \mathrm{E}-08 \\
7.17 \mathrm{E}-08 \\
8.73 \mathrm{E}-08\end{array}$ & $\begin{array}{l}1.49 \mathrm{E}-08 \\
1.69 \mathrm{E}-08 \\
2.79 \mathrm{E}-08 \\
3.39 \mathrm{E}-08\end{array}$ & $\begin{array}{l}7.47 \mathrm{E}-09 \\
8.49 \mathrm{E}-09 \\
1.41 \mathrm{E}-08 \\
1.71 \mathrm{E}-08\end{array}$ & $\begin{array}{l}77 \mathrm{E}-09 \\
44 \mathrm{E}-09 \\
03 \mathrm{E}-09 \\
09 \mathrm{E}-08\end{array}$ & \\
\hline & $\begin{array}{l}3.73 E-06 \\
1.92 E-06 \\
1.16 E-06 \\
8.74 E-07\end{array}$ & $\begin{array}{l}7.50 E-07 \\
3.78 E-07 \\
2.19 E-07 \\
1.60 E-07\end{array}$ & $\begin{array}{l}3.54 \mathrm{E}-07 \\
1.78 \mathrm{E}-07 \\
1.02 \mathrm{E}-07 \\
7.42 \mathrm{E}-08\end{array}$ & $\begin{array}{l}2.18 \mathrm{E}-07 \\
1.09 \mathrm{E}-07 \\
6.21 \mathrm{E}-08 \\
4.52 \mathrm{E}-08\end{array}$ & $\begin{array}{l}1.53 E-07 \\
7.62 E-08 \\
4.32 E-08 \\
3.14 E-08\end{array}$ & $\begin{array}{l}7.46 \mathrm{E}-08 \\
3.72 \mathrm{E}-08 \\
2.10 \mathrm{E}-08 \\
1.52 \mathrm{E}-08\end{array}$ & $\begin{array}{l}2.88 \mathrm{E}-08 \\
1.44 \mathrm{E}-08 \\
8.10 \mathrm{E}-09 \\
5.86 \mathrm{E}-09\end{array}$ & $\begin{array}{l}1.45 \mathrm{E}-08 \\
7.23 \mathrm{E}-09 \\
4.08 \mathrm{E}-09 \\
2.95 \mathrm{E}-09\end{array}$ & $\begin{array}{l}9.26 \mathrm{E}-09 \\
4.64 \mathrm{E}-09 \\
2.62 \mathrm{E}-09 \\
1.90 \mathrm{E}-09\end{array}$ & $\begin{array}{l}1.88 \\
1.36\end{array}$ \\
\hline & $\begin{array}{l}1.16 \\
2.98 \\
5.42 \\
4.21\end{array}$ & $\begin{array}{l}2.11 E-07 \\
6.02 E-07 \\
1.12 E-06 \\
8.85 E-07\end{array}$ & $\begin{array}{l}9.79 \mathrm{E}-08 \\
2.86 \mathrm{E}-07 \\
5.35 \mathrm{E}-07 \\
4.24 \mathrm{E}-07\end{array}$ & $\begin{array}{l}5.98 E-08 \\
1.76 E-07 \\
3.31 E-07 \\
2.63 E-07\end{array}$ & $\begin{array}{l}4.17 E-08 \\
1.24 E-07 \\
2.32 E-07 \\
1.85 E-07\end{array}$ & $\begin{array}{l}2.03 \mathrm{E} \\
6.08 \mathrm{E} \\
1.15 \mathrm{E}\end{array}$ & $\begin{array}{l}7.88 \mathrm{E} \\
2.37 \mathrm{E} \\
4.47 \mathrm{E} \\
3.57 \mathrm{E}\end{array}$ & $\begin{array}{l}3.99 \\
1.20 \\
2.26 \\
1.81\end{array}$ & $\begin{array}{l}7.6 \\
1.4 \\
1.1\end{array}$ & \\
\hline
\end{tabular}


Table A-4. Annual Average Dispersion Factor Around the 400 Area During 1997 for a 10-Meter Release Height.

\begin{tabular}{|c|c|c|c|c|c|c|c|c|c|c|}
\hline Dist: & $0.8 \mathrm{~km}$ & $2.4 \mathrm{~km}$ & $4.0 \mathrm{~km}$ & $5.6 \mathrm{~km}$ & $7.2 \mathrm{~km}$ & $12 \mathrm{~km}$ & $24 \mathrm{~km}$ & $40 \mathrm{~km}$ & $56 \mathrm{~km}$ & $72 \mathrm{~km}$ \\
\hline \multicolumn{11}{|l|}{ Dir: } \\
\hline NNE & $\begin{array}{l}3.78 E-06 \\
3.94 E-06 \\
2.82 E-06 \\
1.78 E-06\end{array}$ & $\begin{array}{l}7.69 \mathrm{E}-07 \\
7.83 \mathrm{E}-07 \\
5.74 \mathrm{E}-07 \\
3.63 \mathrm{E}-07\end{array}$ & $\begin{array}{l}3.65 \mathrm{E}-07 \\
3.70 \mathrm{E}-07 \\
2.73 \mathrm{E}-07 \\
1.73 \mathrm{E}-07\end{array}$ & $\begin{array}{l}2.26 \mathrm{E}-07 \\
2.28 \mathrm{E}-07 \\
1.69 \mathrm{E}-07 \\
1.07 \mathrm{E}-07\end{array}$ & $\begin{array}{l}1.58 \mathrm{E}-07 \\
1.60 \mathrm{E}-07 \\
1.18 \mathrm{E}-07 \\
7.49 \mathrm{E}-08\end{array}$ & $\begin{array}{l}7.79 \mathrm{E}-08 \\
7.84 \mathrm{E}-08 \\
5.81 \mathrm{E}-08 \\
3.68 \mathrm{E}-08\end{array}$ & $\begin{array}{l}3.04 \mathrm{E}-08 \\
3.05 \mathrm{E}-08 \\
2.27 \mathrm{E}-08 \\
1.43 \mathrm{E}-08\end{array}$ & $\begin{array}{l}1.54 E-08 \\
1.54 E-08 \\
1.15 E-08 \\
7.24 E-09\end{array}$ & $\begin{array}{l}9.88 \mathrm{E}-09 \\
9.88 \mathrm{E}-09 \\
7.36 \mathrm{E}-09 \\
4.65 \mathrm{E}-09\end{array}$ & $\begin{array}{l}7.11 \mathrm{E}-09 \\
7.10 \mathrm{E}-09 \\
5.29 \mathrm{E}-09 \\
3.34 \mathrm{E}-09\end{array}$ \\
\hline $\begin{array}{l}\text { SE } \\
\text { SSE }\end{array}$ & $\begin{array}{l}1.98 \mathrm{E}-06 \\
2.30 \mathrm{E}-06 \\
3.95 \mathrm{E}-06 \\
3.37 \mathrm{E}-06\end{array}$ & $\begin{array}{l}3.93 \mathrm{E}-07 \\
4.53 \mathrm{E}-07 \\
7.82 \mathrm{E}-07 \\
6.87 \mathrm{E}-07\end{array}$ & $\begin{array}{l}1.85 \mathrm{E}-07 \\
2.13 \mathrm{E}-07 \\
3.68 \mathrm{E}-07 \\
3.26 \mathrm{E}-07\end{array}$ & $\begin{array}{l}1.14 \mathrm{E}-07 \\
1.31 \mathrm{E}-07 \\
2.26 \mathrm{E}-07 \\
2.01 \mathrm{E}-07\end{array}$ & $\begin{array}{l}7.98 \mathrm{E}-08 \\
9.12 \mathrm{E}-08 \\
1.58 \mathrm{E}-07 \\
1.41 \mathrm{E}-07\end{array}$ & $\begin{array}{l}3.90 E-08 \\
4.44 E-08 \\
7.68 E-08 \\
6.89 E-08\end{array}$ & $\begin{array}{l}1.51 \mathrm{E}-08 \\
1.71 \mathrm{E}-08 \\
2.96 \mathrm{E}-08 \\
2.68 \mathrm{E}-08\end{array}$ & $\begin{array}{l}7.61 \mathrm{E}-09 \\
8.56 \mathrm{E}-09 \\
1.48 \mathrm{E}-08 \\
1.35 \mathrm{E}-08\end{array}$ & $\begin{array}{l}4.88 \mathrm{E}-09 \\
5.47 \mathrm{E}-09 \\
9.44 \mathrm{E}-09 \\
8.66 \mathrm{E}-09\end{array}$ & $\begin{array}{l}3.50 \mathrm{E}-09 \\
3.92 \mathrm{E}-09 \\
6.76 \mathrm{E}-09 \\
6.22 \mathrm{E}-09\end{array}$ \\
\hline $\begin{array}{l}\text { S } \\
\text { SSW } \\
\text { SW } \\
\text { WSW }\end{array}$ & $\begin{array}{l}2.77 \mathrm{E}-06 \\
2.28 \mathrm{E}-06 \\
1.81 \mathrm{E}-06 \\
1.24 \mathrm{E}-06\end{array}$ & $\begin{array}{l}5.72 \mathrm{E}-07 \\
4.59 \mathrm{E}-07 \\
3.58 \mathrm{E}-07 \\
2.42 \mathrm{E}-07\end{array}$ & $\begin{array}{l}2.72 \mathrm{E}-07 \\
2.16 \mathrm{E}-07 \\
1.69 \mathrm{E}-07 \\
1.14 \mathrm{E}-07\end{array}$ & $\begin{array}{l}1.68 \mathrm{E}-07 \\
1.33 \mathrm{E}-07 \\
1.04 \mathrm{E}-07 \\
6.97 \mathrm{E}-08\end{array}$ & $\begin{array}{l}1.18 \mathrm{E}-07 \\
9.30 \mathrm{E}-08 \\
7.26 \mathrm{E}-08 \\
4.87 \mathrm{E}-08\end{array}$ & $\begin{array}{l}5.78 \mathrm{E}-08 \\
4.54 \mathrm{E}-08 \\
3.56 \mathrm{E}-08 \\
2.37 \mathrm{E}-08\end{array}$ & $\begin{array}{l}2.25 \mathrm{E}-08 \\
1.76 \mathrm{E}-08 \\
1.39 \mathrm{E}-08 \\
9.18 \mathrm{E}-09\end{array}$ & $\begin{array}{l}1.14 \mathrm{E}-08 \\
8.88 \mathrm{E}-09 \\
7.02 \mathrm{E}-09 \\
4.62 \mathrm{E}-09\end{array}$ & $\begin{array}{l}7.30 \mathrm{E}-09 \\
5.70 \mathrm{E}-09 \\
4.51 \mathrm{E}-09 \\
2.96 \mathrm{E}-09\end{array}$ & $\begin{array}{l}5.26 \mathrm{E}-09 \\
4.10 \mathrm{E}-09 \\
3.25 \mathrm{E}-09 \\
2.13 \mathrm{E}-09\end{array}$ \\
\hline $\begin{array}{l}\text { W } \\
\text { WWW } \\
\text { NW }\end{array}$ & $\begin{array}{l}1.30 E-06 \\
1.26 E-06 \\
2.24 E-06 \\
3.31 E-06\end{array}$ & $\begin{array}{l}2.51 \mathrm{E}-07 \\
2.41 \mathrm{E}-07 \\
4.31 \mathrm{E}-07 \\
6.62 \mathrm{E}-07\end{array}$ & $\begin{array}{l}1.18 \mathrm{E}-07 \\
1.13 \mathrm{E}-07 \\
2.01 \mathrm{E}-07 \\
3.13 \mathrm{E}-07\end{array}$ & $\begin{array}{l}7.25 E-08 \\
6.92 E-08 \\
1.23 E-07 \\
1.93 E-07\end{array}$ & $\begin{array}{l}5.07 E-08 \\
4.83 E-08 \\
8.59 E-08 \\
1.35 E-07\end{array}$ & $\begin{array}{l}2.47 \mathrm{E}-08 \\
2.36 \mathrm{E}-08 \\
4.17 \mathrm{E}-08 \\
6.60 \mathrm{E}-08\end{array}$ & $\begin{array}{l}9.58 \mathrm{E}-09 \\
9.11 \mathrm{E}-09 \\
1.61 \mathrm{E}-08 \\
2.56 \mathrm{E}-08\end{array}$ & $\begin{array}{l}4.82 \mathrm{E}-09 \\
4.59 \mathrm{E}-09 \\
8.06 \mathrm{E}-09 \\
1.29 \mathrm{E}-08\end{array}$ & $\begin{array}{l}3.09 \mathrm{E}-09 \\
2.95 \mathrm{E}-09 \\
5.15 \mathrm{E}-09 \\
8.28 \mathrm{E}-09\end{array}$ & $\begin{array}{l}2.22 \mathrm{E}-09 \\
2.12 \mathrm{E}-09 \\
3.70 \mathrm{E}-09 \\
5.95 \mathrm{E}-09\end{array}$ \\
\hline
\end{tabular}


DOE/RL-98-33

Table A-5. Radionuclide Data Used for CAP88-PC Dose Calculations at Hanford.

(1 of 3 sheets)

\begin{tabular}{lcccc} 
Nuclide & $\begin{array}{c}\text { Clearance } \\
\text { Class }\end{array}$ & $\begin{array}{c}\text { Particle } \\
\text { Size } \\
\text { (microns) }\end{array}$ & $\begin{array}{c}\text { Scavenging } \\
\text { Coefficient } \\
\text { (per second) }\end{array}$ & $\begin{array}{c}\text { Dry } \\
\text { Deposition } \\
\text { Velocity } \\
\text { (m/s) }\end{array}$ \\
\hline H-3 & $*$ & 0.0 & $0.00 \mathrm{E}+00$ & $0.00 \mathrm{E}+00$ \\
C0-60 & Y & 1.0 & $1.60 \mathrm{E}-06$ & $1.80 \mathrm{E}-03$ \\
SR-90 & 0 & 1.0 & $1.60 \mathrm{E}-06$ & $1.80 \mathrm{E}-03$ \\
SB-125 & W & 1.0 & $1.60 \mathrm{E}-06$ & $1.80 \mathrm{E}-03$ \\
I-129 & D & 1.0 & $1.60 \mathrm{E}-06$ & $3.50 \mathrm{E}-02$ \\
CS-137 & D & 1.0 & $1.60 \mathrm{E}-06$ & $1.80 \mathrm{E}-03$ \\
BA-137M & D & 1.0 & $1.60 \mathrm{E}-06$ & $1.80 \mathrm{E}-03$ \\
PU-238 & Y & 1.0 & $1.60 \mathrm{E}-06$ & $1.80 \mathrm{E}-03$ \\
PU-239 & Y & 1.0 & $1.60 \mathrm{E}-06$ & $1.80 \mathrm{E}-03$ \\
PU-241 & $Y$ & 1.0 & $1.60 \mathrm{E}-06$ & $1.80 \mathrm{E}-03$ \\
AM-241. & W & 1.0 & $1.60 \mathrm{E}-06$ & $1.80 \mathrm{E}-03$ \\
PB-212 & $D$ & 1.0 & $1.60 \mathrm{E}-06$ & $1.80 \mathrm{E}-03$ \\
RN-222 & $*$ & 0.0 & $0.00 \mathrm{E}+00$ & $0.00 \mathrm{E}+00$
\end{tabular}


DOE/RL-98-33

Table A-5. Radionuclide Data Used for CAP88-PC Dose Calculations at Hanford.

(2 of 3 sheets)

\begin{tabular}{|c|c|c|c|c|c|}
\hline \multirow[b]{2}{*}{ Nuclide } & \multicolumn{3}{|c|}{ DECAY CONSTANT (PER DAY) } & \multicolumn{2}{|c|}{ TRANSFER COEFFICIENT } \\
\hline & $\begin{array}{l}\text { Radio- } \\
\text { active (1) }\end{array}$ & Surface & Water & Milk (2) & Meat (3) \\
\hline $\begin{array}{l}H-3 \\
C O-60 \\
\text { SR-90 } \\
\text { RU-106 } \\
\text { SN-113 } \\
\text { SB-125 } \\
I-129 \\
C S-134 \\
C S-137 \\
\text { BA-137M } \\
\text { PU-238 } \\
\text { PU-239 } \\
\text { PU-241 } \\
\text { AM-241 }\end{array}$ & $\begin{array}{l}0.00 \mathrm{E}+00 \\
0.00 \mathrm{E}+00 \\
0.00 \mathrm{E}+00 \\
0.00 \mathrm{E}+00 \\
0.00 \mathrm{E}+00 \\
0.00 \mathrm{E}+00 \\
0.00 \mathrm{E}+00 \\
0.00 \mathrm{E}+00 \\
0.00 \mathrm{E}+00 \\
3.91 \mathrm{E}+02 \\
0.00 \mathrm{E}+00 \\
0.00 \mathrm{E}+00 \\
0.00 \mathrm{E}+00 \\
0.00 \mathrm{E}+00\end{array}$ & $\begin{array}{l}5.48 \mathrm{E}-05 \\
5.48 \mathrm{E}-05 \\
5.48 \mathrm{E}-05 \\
5.48 \mathrm{E}-05 \\
5.48 \mathrm{E}-05 \\
5.48 \mathrm{E}-05 \\
5.48 \mathrm{E}-05 \\
5.48 \mathrm{E}-05 \\
5.48 \mathrm{E}-05 \\
5.48 \mathrm{E}-05 \\
5.48 \mathrm{E}-05 \\
5.48 \mathrm{E}-05 \\
5.48 \mathrm{E}-05 \\
5.48 \mathrm{E}-05\end{array}$ & $\begin{array}{l}0.00 \mathrm{E}+00 \\
0.00 \mathrm{E}+00 \\
0.00 \mathrm{E}+00 \\
0.00 \mathrm{E}+00 \\
0.00 \mathrm{E}+00 \\
0.00 \mathrm{E}+00 \\
0.00 \mathrm{E}+00 \\
0.00 \mathrm{E}+00 \\
0.00 \mathrm{E}+00 \\
0.00 \mathrm{E}+00 \\
0.00 \mathrm{E}+00 \\
0.00 \mathrm{E}+00 \\
0.00 \mathrm{E}+00 \\
0.00 \mathrm{E}+00\end{array}$ & $\begin{array}{l}0.00 \mathrm{E}+00 \\
2.00 \mathrm{E}-03 \\
1.50 \mathrm{E}-03 \\
6.00 \mathrm{E}-07 \\
1.00 \mathrm{E}-03 \\
1.00 \mathrm{E}-04 \\
1.00 \mathrm{E}-02 \\
7.00 \mathrm{E}-03 \\
7.00 \mathrm{E}-03 \\
3.50 \mathrm{E}-04 \\
1.00 \mathrm{E}-07 \\
1.00 \mathrm{E}-07 \\
1.00 \mathrm{E}-07 \\
4.00 \mathrm{E}-07\end{array}$ & $\begin{array}{r}0.00 \mathrm{E}+00 \\
2.00 \mathrm{E}-02 \\
3.00 \mathrm{E}-04 \\
2.00 \mathrm{E}-03 \\
8.00 \mathrm{E}-02 \\
1.00 \mathrm{E}-03 \\
7.00 \mathrm{E}-03 \\
2.00 \mathrm{E}-02 \\
2.00 \mathrm{E}-02 \\
1.50 \mathrm{E}-04 \\
5.00 \mathrm{E}-07 \\
5.00 \mathrm{E}-07 \\
5.00 \mathrm{E}-07 \\
3.50 \mathrm{E}-06\end{array}$ \\
\hline $\begin{array}{l}8-212 \\
N-222\end{array}$ & $\begin{array}{l}1.56 \mathrm{E}+00 \\
1.81 \mathrm{E}-01\end{array}$ & $\begin{array}{l}5.48 \mathrm{E}-05 \\
5.48 \mathrm{E}-05\end{array}$ & $\begin{array}{l}0.00 E+00 \\
0.00 E+00\end{array}$ & $\begin{array}{l}2.50 \mathrm{E}-04 \\
0.00 \mathrm{E}+00\end{array}$ & $\begin{array}{l}3.00 E-04 \\
0.00 E+00\end{array}$ \\
\hline
\end{tabular}

F0OTNOTES: (1) Effective radioactive decay constant in plume:

set to zero if less than $1.0 E-2$

(2) Fraction of animal's daily intake of nuclide which appears in each $L$ of $\mathrm{milk}$ (days $/ L$ )

(3) Fraction of animal's daily intake of nuclide which appears in each $\mathrm{kg}$ of meat (days $/ \mathrm{kg}$ ) 
DOE/RL-98-33

Table A-5. Radionuclide Data Used for CAP88-PC Dose Calculations at Hanford.

( 3 of 3 sheets)

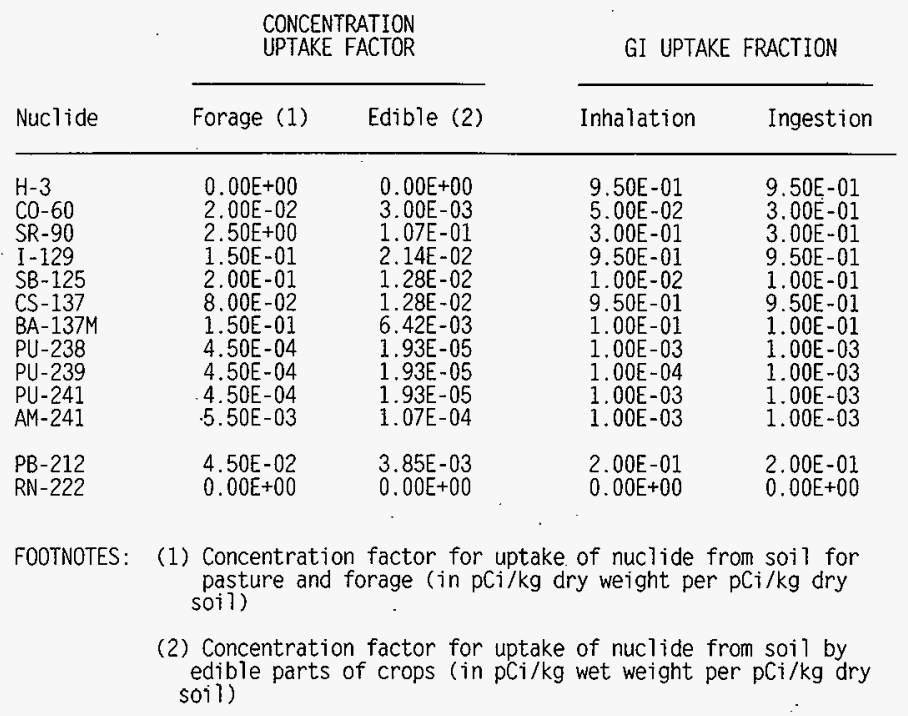


Table A-6. Exposure and Consumption Data for the Hanford Site.

$$
\text { (1 of } 2 \text { sheets) }
$$

FOOD SOURCE FOR THE MAXIMALLY EXPOSED INDIVIDUAL:

$\begin{array}{rccc} & \text { Local } & \text { Regional } & \text { Imported } \\ \text { Vegetable: } & 1.000 & 0.000 & 0.000 \\ \text { Meat: } & 1.000 & 0.000 & 0.000 \\ \text { Milk: } & 1.000 & 0.000 & 0.000\end{array}$

\section{VALUES FOR RADIONUCLIDE-INDEPENDENT VARIABLES:}

HUMAN INHALATION RATE

Cubic centimeters/hr $\quad 9.70 \mathrm{E}+05$

SOIL PARAMETERS

Effective surface density ( $\mathrm{kg} / \mathrm{sq} \mathrm{m}$, dry weight)

(Assumes $15 \mathrm{~cm}$ plow layer) $2.24 \mathrm{E}+02$

BUILDUP TIMES

For activity in soil (years) $\quad 5.00 \mathrm{E}+01$

For radionuclides deposited on ground/water (days) $\quad 1.83 \mathrm{E}+04$

DELAY TIMES

Ingestion of pasture grass by animals (hr)

$0.00 \mathrm{E}+00$

Ingestion of stored feed by animals (hr)

Ingestion of leafy vegetables by man ( $h r$ )

Ingestion of produce by man (hr)

Transport time from animal feed-mi lk-man (day)

2. $40 \mathrm{E}+03$

$2.40 \mathrm{E}+01$

$1.20 \mathrm{E}+02$

2. $00 \mathrm{E}+00$

Time from s.laughter to consumption (day)

1. $50 \mathrm{E}+01$

WEATHERING

Removal rate constant for physical loss (per hr)

$3.00 \mathrm{E}-03$

CROP EXPOSURE DURATION

Pasture grass (hr)

Crops/leafy vegetables (hr)

$7.20 E+02$

2. $16 \mathrm{E}+03$

AGRICULTURAL PRODUCTIVITY

Grass-cow-mi lk-man pathway $(\mathrm{kg} / \mathrm{sq} \mathrm{m}$ )

Produce/leafy veg for human consumption $(\mathrm{kg} / \mathrm{sq} \mathrm{m})$

3. $00 \mathrm{E}-01$

$2.00 E+00$ 
Table A-6. Exposure and Consumption Data for the Hanford Site.

(2 of 2 sheets)

FALLOUT INTERCEPTION FRACTIONS

Vegetables

2.50E-01

Pasture

$2.50 \mathrm{E}-01$

GRAZING PARAMETERS

Fraction of year animals graze on pasture

Fraction of daily feed that is pasture grass

when animal grazes on pasture

7.50E-01

$1.00 E+00$

ANIMAL FEED CONSUMPTION FACTORS

Contaminated feed/forage (kg/day, dry weight) $\quad 1.56 \mathrm{E}+01$

DAIRY PRODUCTIVITY

Milk production of cow ( $L /$ day)

1. $10 \mathrm{E}+01$

MEAT ANIMAL SLAUGHTER PARAMETERS

Muscle mass of animal at slaughter $(\mathrm{kg})$

Fraction of herd slaughtered (per day)

$2.00 \mathrm{E}+02$

$3.81 \mathrm{E}-03$

DECONTAMINATION

Fraction of radioactivity retained after washing

for leafy vegetables and produce

$1.00 E+00$

FRACTIONS GROWN IN GARDEN OF INTEREST

Produce ingested

Leafy vegetables ingested

$1.00 E+00$

$1.00 \mathrm{E}+00$

INGESTION RATIOS:

IMMEDIATE SURROUNDING AREA/TOTAL WITHIN AREA

Vegetables

Meat

$1.00 E+00$

Milk

$1.00 E+00$

$1.00 E+00$

MINIMUM INGESTION FRACTIONS FROM OUTSIDE AREA

(Minimum fractions of food types from outside

area listed below are actual fixed values.) Vegetables

Meat

$0.00 E+00$

Milk

$0.00 \mathrm{E}+00$

$0.00 \mathrm{E}+00$

HUMAN FOOD UTILIZATION FACTORS

Produce ingestion $(\mathrm{kg} / \mathrm{y})$

Milk ingestion $(\mathrm{L} / \mathrm{y})$

Meat ingestion $(\mathrm{kg} / \mathrm{y})$

Leafy vegetable ingestion $(\mathrm{kg} / \mathrm{y})$

2. $20 \mathrm{E}+02$

$2.70 \mathrm{E}+02$

$9.80 \mathrm{E}+01$

3. $00 \mathrm{E}+01$

SWIMMING PARAMETERS

Fraction of time spent swimming

Di lution factor for water $(\mathrm{cm})$

1.00E-02

1. $00 \mathrm{E}+00$ 
DOE/RL-98-33

Table A-7. Hanford Site Meteorological Data - General Site Information.

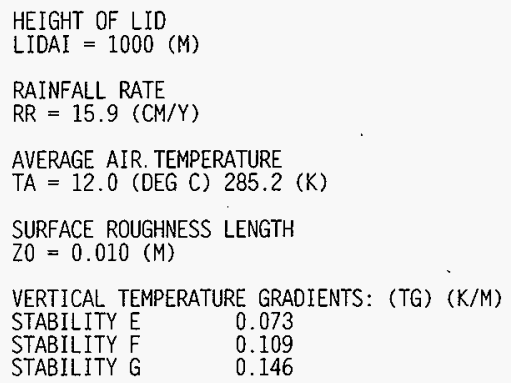




\section{DISTRIBUTION}

Number of Copies

OFFSITE

U.S. Environmental Protection Agency, Region 10 1200 Sixth Avenue

Seattle, WA 98101

G. M. Leitch (5)

AT-081

A. Frankel

R. W. Poeten

AT-081

6

Washington State Department of Health

Air Emissions and Defense Waste Section

Division of Radiation Protection

P. O. Box 47827

Olympia, WA $98504-7827$

A. W. Conklin (5)

J. E. Erickson

Washington State Department of Ecology

M. A. Wilson

B5-18

U.S. Department of Energy-Headquarters

R. F. Pelletier (3)

EH-232

M. K. Harmon

EM-442

K. C. Duvall

EH-232

Confederated Tribes of the Umatilla Indian Reservation P. O. Box 638

Pendleton, OR 97801

J. R. Wilkinson 
DISTRIBUTION (continued)

\section{Number of Copies}

\section{OFFSITE}

1

Nez Perce Tribe

Environmental Restoration and Waste Management

P. O. Box 365

Lapwai, ID 83540

D. L. Powaukee

Yakama Indian Nation

Environmental Restoration Waste Management Program

P.O. Box 151

Toppenish, Washington 98948

R. Jim

\section{ONSITE}

16

U.S. Department of Energy

Richland Operations Office

G. M. Bell

A5-52

D. W. Bowser

A2-22

R. F. Brich

H0-12

S. E. Clarke

A5-15

J. B. Hall

A5-15

J. E. Mecca

R3-81

B. M. Pangborn

A5-55

J. E. Rasmussen

A5-15

H. M. Rodriguez (5)

A5-15

D. C. Ward

A. H. Wirkkala

A5-15

DOE-RL Public Reading Room 
DISTRIBUTION (continued)

\section{Number of Copies}

\section{ONSITE}

M. Y. Ballinger

E. G. Damberg

P7-68

R. L. Dirkes

K6-75

W. T. Farris

$\mathrm{K} 3-54$

R. W. Hanf, Jr.

K6-75

G. R. Hoenes

P7-79

S. J. Jette

P7-68

G. W. McNair

P7.75

T. W. Moon

P7-79

K. Rhoads

K3-54

G. A. Simiele

P7-79

L. H. Staven

K3-54

M. J. Sula

P7-68

H. T. Tilden II

P7-79

R. K. Woodruff

P7-68

PNNL Reference Library

P8-55

Bechtel Hanford, Inc.

R. G. Egge

T7-05

M. E. Greenidge

$\mathrm{X} 5-54$

R. J. Landon

H0-18

D. W. Long

T7-05

M. R. Morton

HO- 18

J. E. Rugg

J. G. Woolard

S3-21

J. P. Zoric

HO- 17

X5-57

Fluor Daniel Hanford, Inc.

President's Office

H5-20

W. D. Adair

H6-21

J. A. Bates

H6-23

S. M. Price

H6-23

D. G. Ranade

H6-23

B. D. Williamson

B3-15 


\section{DISTRIBUTION (continued)}

\section{Number of Copies}

\section{ONSITE}

CH2M Hill, Inc.

E. T. Coenenberg

H9-11

10

B\&W Hanford Company

J. M. Barnett

L1-05

T. G. Beam

S6-51

J. E. Bramson

T5-54

N. R. Dahl

N2-57

T. A. Dillhoff

N2-57

D. L. Johnson

S6-01

G. J. LeBaron

S6-19

D. E. Rasmussen

$\mathrm{N} 1-47$

R. D. Weissenfels

S6-51

D. L. Wiegand

T5-54

2

DE\&S Hanford, Inc.

R. G. Gant

X3-79

D. J. Watson

X3-79

1

DynCorp Tri-Cities Services, Inc.

B. J. Dixon

B4-20

11

Lockheed Martin Hanford Corporation

M. S. Allen

S7-01

D. J. Carrell

R1-51

G. M. Crummel

D. L. Dyekman

R1-51

K. A. Elsethagen

T4-08

B. G. Erlandson

S5-03

P. C. Miller

R2-36

R. P. Raven

R1-51

K. S. Tollefson

R1-51

R1-51 


\section{DISTRIBUTION (continued)}

\section{Number of Copies}

\section{ONSITE}

Lockheed Martin Services, Inc.

\section{Central Files}

Numatec Hanford Corporation

R. A. Kaldor

H5-25

Waste Management Federal Services of Hanford, Inc.

B. M. Barnes

T4-04

R. J. Boom

T6-12

M. W. Bowman

S6-72

H. C. Boynton

T4-52

J. R. Buckley

T3-04

B. L. Curn

H6-36

W. E. Davis

H6-36

L. P. Diediker (75)

H6-36

D. P. Fasset

T3-28

D. L. Flyckt

S6-71

T. P. Frazier

H6-25

D. W. Fritz

H6-36

B. P. Gleckler

H6-36

W. E. Green

H6-36

E. M. Greager

H6-36

R. D. Haggard

H6-25

D. L. Halgren

L6-04

J. S. Hill

H6-25

N. A. Homan

H6-25

R. E. Johnson

H6-25

L. D. Kamberg

J. J. Luke

H6-25

J. A. Morrison

S3-31

J. K. Perry

H6-25

D. L. Renberger

T3-03

R. W. Szelmeczka

L6-05

D. B. Van Leuven

H6-10

G. T. Wells

H6-36

J. A. Winterhalder 


\section{DISTRIBUTION (continued)}

\section{Number of Copies}

\section{ONSITE}

5

Waste Management Federal

Services, Inc. Northwest Operations

J. J. Dorian

H1-13
A. R. Johnson
B. M. Markes
S. J. McKinney
C. J. Perkins

H1-13

H1-13

H1-12

H1-12 


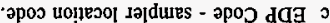

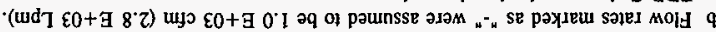

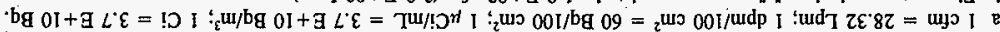

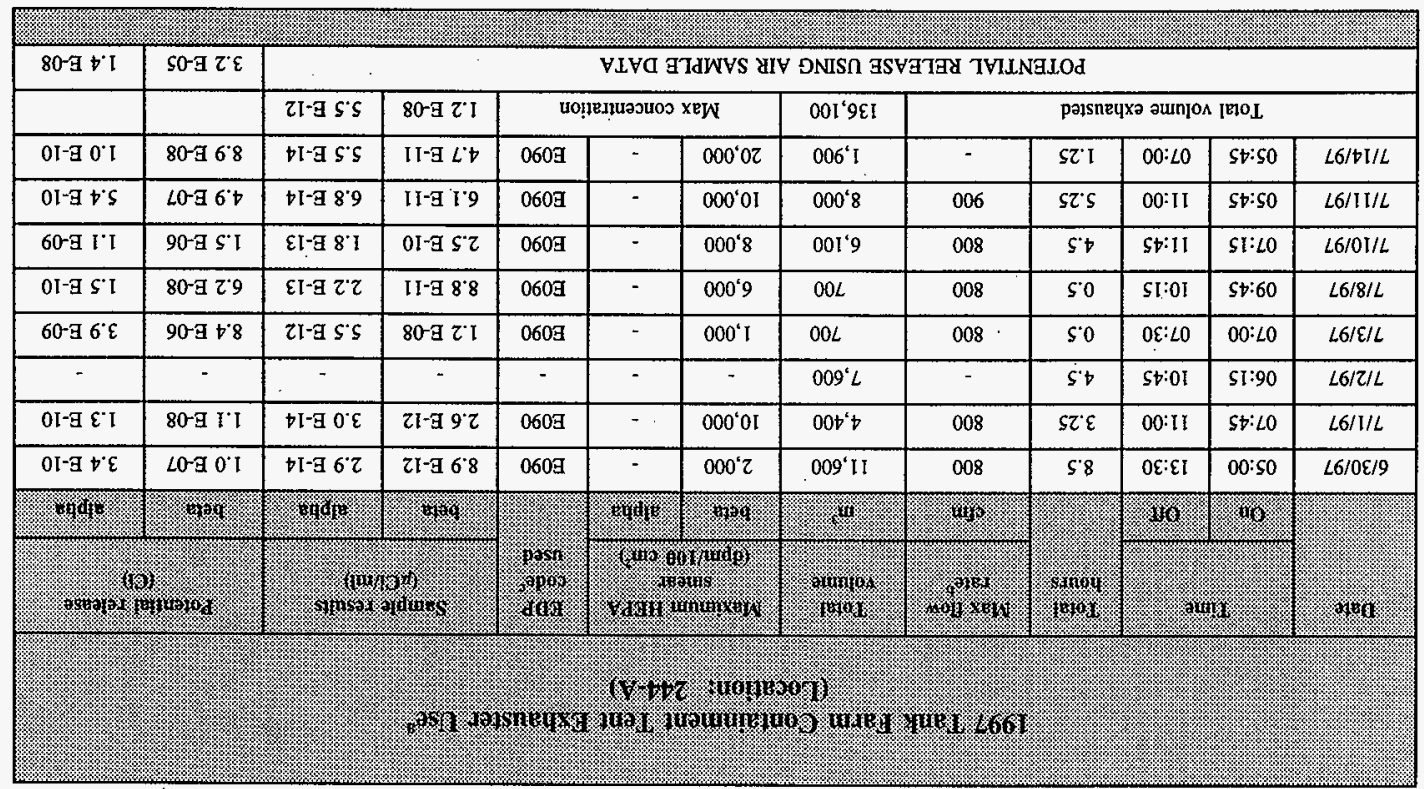




\subsubsection{NOC for the 3732 Process Equipment Development Laboratory Demolition}

The Notice of Construction for the 3732 Process Equipment Development Laboratory Demolition activity requires concentrations from the near-facility ambient air monitoring stations be reported in the annual Radionuclide Air Emissions Report for the Hanford Site. The information provided in Tables 5-4 and 5-5 fulfills this requirement.

Table 5-4

\begin{tabular}{|c|c|c|c|c|c|}
\hline & 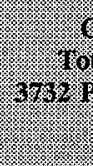 & 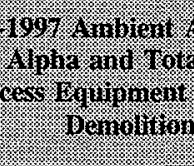 & 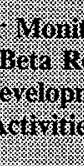 & 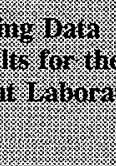 & 1 \\
\hline 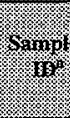 & Sampine & 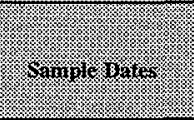 & 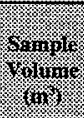 & ring & 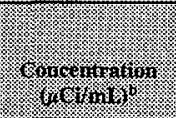 \\
\hline \multirow[t]{2}{*}{ N490 } & S235381 & $9 / 16 / 97$ to $9 / 26 / 97$ & 788 & $\begin{array}{l}\text { total alpha } \\
\text { total beta }\end{array}$ & $\begin{array}{l}9.9 \mathrm{E}-16 \\
6.4 \mathrm{E}-15\end{array}$ \\
\hline & $\$ 236572$ & $9 / 26 / 97$ to $10 / 01 / 97$ & 397 & $\begin{array}{l}\text { total alpha } \\
\text { total beta }\end{array}$ & $\begin{array}{l}3.0 \mathrm{E}-15 \\
5.0 \mathrm{E}-14\end{array}$ \\
\hline \multirow[t]{2}{*}{ N491 } & S236570 & $9 / 16 / 97$ to $9 / 26 / 97$ & 768 & $\begin{array}{l}\text { total alpha } \\
\text { total beta }\end{array}$ & $\begin{array}{l}2.6 \mathrm{E}-15 \\
2.0 \mathrm{E}-14\end{array}$ \\
\hline & $\$ 235382$ & $9 / 26 / 97$ to $10 / 01 / 97$ & 406 & $\begin{array}{l}\text { total alpha } \\
\text { total beta }\end{array}$ & $\begin{array}{l}3.9 \mathrm{E}-15 \\
1.1 \mathrm{E}-14\end{array}$ \\
\hline
\end{tabular}

Notes:

a ID $=$ Identification, i.e., the alpha-numeric designator for the respective sampler.

b $1 \mu \mathrm{Ci}=3.7 \mathrm{E}+04 \mathrm{bq} ; 1 \mathrm{~mL}=1.0 \mathrm{E}-06 \mathrm{~m}^{3}$. 
Table 5-5

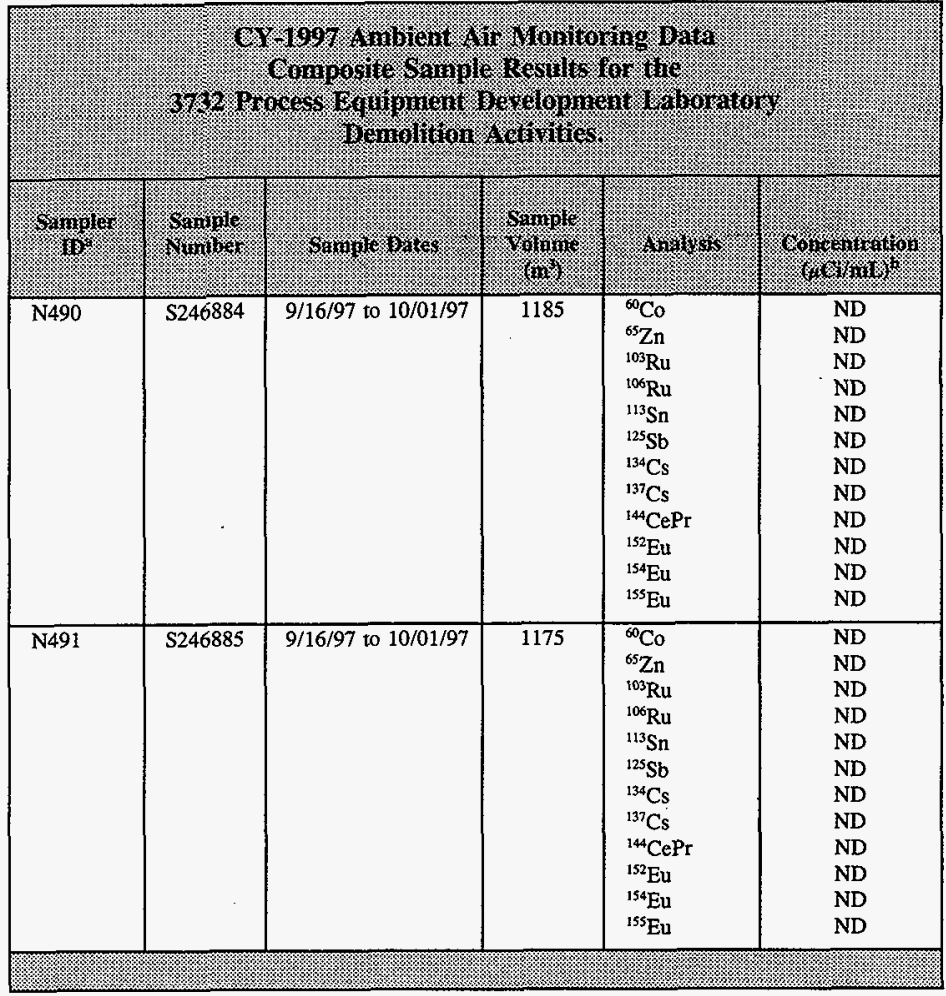

Notes:

a $\quad$ ID $=$ Identification, i.e., the alpha-numeric designator for the respective sampler.

b $1 \mu \mathrm{Ci}=3.7 \mathrm{E}+04 \mathrm{bq} ; 1 \mathrm{~mL}=1.0 \mathrm{E}-06 \mathrm{~m}^{3}$.

\subsubsection{NOC for the Excavation and Backfilling Activities in Support of a Potable Water Line Repair}

The Notice of Construction for the Excavation and Backfilling Activities in Support of a Potable Water Line Repair requires that a summary of the radiological survey data be reported in the annual Radionuclide Air Emissions Report for the Hanford Site. Speck beta and gamma contamination was found on broken asphalt and concrete and on one piece of old 
water pipe. The greatest field reading was $60,000 \mathrm{dpm}$. No alpha contamination was detected. No contamination was detected in the soil. The contamination specks were packaged and are awaiting final disposal.

\subsubsection{6-Z-10 Stack Emissions}

The estimated emissions for the 296-Z-10 stack are provided in Table 5-6. Record sampling for this stack was discontinued during June of 1987 ,... because emissions were frequently below detection limits and because the potential for exceeding $4 \%$ of the Derived Concentration Guides (DCGs) was minimal (refer to DOE Order 5400.5 and WHC 1988). During 1997 , this stack operated intermittently to supply building ventilation to the office areas of the 231-Z Building. The emissions estimate provided in Table 5-6 is considered to be a conservative estimate, based on historical emissions from this point source.

Table 5-6

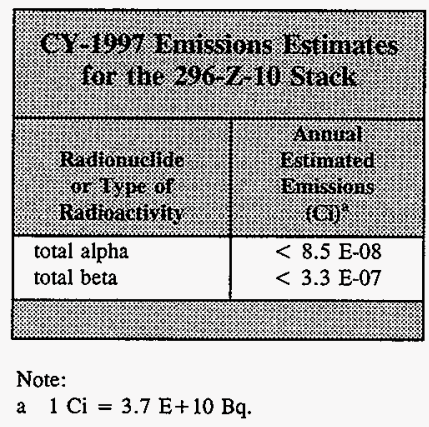

\subsection{QUALITY ASSURANCE PROGRAMS' COMPLIANCE STATUS}

The document Quality Assurance Program for Radionuclide Airborne Emissions Monitoring (WHC 1995b) contain appendices that provide point-by-point comparisons of the major point source monitoring systems to the quality assurance criteria of 40 CFR 61 , Appendix B Method 114. The document Effluent Monitoring Quality Assurance Project Plan for Radioactive Airborne Emissions Data (WHC 1995a) contains the quality assurance activities associated with data collection and reporting. FDH and BHI airborne effluent monitoring activities are conducted in accordance with federal and state regulations as well as the documents listed below. 
PNNL has implemented a quality assurance plan for facility effluent monitoring, as required by 40 CFR 61, Appendix B, Method 114. The effluent monitoring quality assurance plan incorporates guidance from the documents listed in the paragraph below.

The quality assurance programs described by these plans are based on the following documentation:

- EPA QAMS-005/80, Interim Guidelines for Preparing Quality Assurance Project Plans, (EPA 1983)

- DOE 5700.6C, Quality Assurance

- DOE 5400.1, General Environmental Protection Program

- DOE/EH-0173T, Environmental Regulatory Guide for Radiological Effluent Monitoring and Environmental Surveillance, (DOE 1991)

- applicable criteria of ASME NQA-1, Quality Assurance Requirements for Nuclear Facilities, (ASME 1989). 


\subsection{REFERENCES}

ASME, 1989, Quality Assurance Program for Nuclear Facilities, American Society for Testing and Material, NQA-1-1989 Edition, New York.

Brown, R. M., G. L. Ogram, and F. S. Spencer, 1990, Oxidation and Dispersion of HT in the Environment: The August 1986 Field Experiment at Chalk River, Health Physics 58(2):171-181.

DOE, 1991, Environmental Regulatory Guide for Radiological Effluent Monitoring and Environmental Surveillance, DOE/EH-0173T, U.S. Department of Energy, Washington D.C.

DOE-RL, 1988, Hanford Site Dangerous Waste Part A Permit Application, DOE/RL-88-21, U.S. Department of Energy, Richland Operations Office, Richland, Washington.

DOE-RL, 1991, Calendar Year 1990 Air Emissions Report for the Hanford Site, DOE/RL-91-10, U.S. Department of Energy, Richland Field Office, Richland, Washington.

DOE-RL, 1992, Radionuclide Air Emissions Report for the Hanford Site, Calendar Year 1991, DOE/RL-92-30, U.S. Department of Energy, Richland Field Office, Richland, Washington.

DOE-RL, 1993, Radionuclide Air Emissions Report for the Hanford Site, Calendar Year 1992, DOE/RL-93-36, U.S: Department of Energy, Richland Field Office, Richland, Washington.

DOE-RL, 1994, Radionuclide Air Emissions Report for the Hanford Site, Calendar Year 1993, DOE/RL-94-51, U.S. Department of Energy, Richland Field Office, Richland, Washington.

DOE-RL, 1995, Radionuclide Air Emissions Report for the Hanford Site, Calendar Year 1994, DOE/RL-95-49, U.S. Department of Energy, Richland Field Office, Richland, Washington.

DOE-RL, 1996, Radionuclide Air Emissions Report for the Hanford Site, Calendar Year 1995, DOE/RL-96-37, U.S. Department of Energy, Richland Field Office, Richland, Washington.

DOE-RL, 1997a, Radionuclide Air Emissions Report for the Hanford Site, Calendar Year 1996, DOE/RL-97-43, U.S. Department of Energy, Richland Field Office, Richland, Washington. 
DOE-RL, 1997b, Radioactive Air Emissions Notice of Construction Portable/Temporary Air Emission Units, DOE/RL-96-75 Rev. 1, U.S. Department of Energy, Richland Field Office, Richland, Washington.

EPA, 1980, Interim Guidelines and Specifications for Preparing Quality Assurance Project Plans, EPA/500/80, U.S. Environmental Protection Agency, Washington, D.C.

EPA, 1992, User's Guide for CAP88-PC, Version 1.0, 402-B-92-001, Office of Radiation Programs, U.S. Environmental Protection Agency, Las Vegas, Nevada.

PNL, 1988, GENII - The Hanford Environmental Radiation Dosimetry Software System, PNL-6584, Vols. 1-3, Pacific Northwest Laboratory, Richland, Washington.

PNL, 1990, Hanford Site Environmental Report for Calendar Year 1989, PNL-7346, Pacific Northwest Laboratory, Richland, Washington.

PNL, 1991, Hanford Area 1990 Population and 50-year Projections, PNL-7.803, Pacific Northwest Laboratory, Richland, Washington.

PNL, 1993, Recommended Environmental Dose Calculation Methods and Hanford-Specific Parameters, PNL-3777, Rev. 2., Pacific Northwest Laboratory, Richland, Washington.

PNL, 1997, Hanford Site National Environmental Policy Act (NEPA) Characterization, PNL-6415 Rev. 9, Pacific Northwest Laboratory, Richland, Washington.

PNNL, 1998a, Hanford Site Environmental Report for Calendar Year 1997, PNNL-11795, Pacific Northwest National Laboratory, Richland, Washington.

PNNL, 1998b, 1997 Surface Environmental Surveillance Data, PNNL-11796, Pacific Northwest National Laboratory, Richland, Washington.

PNNL, 1998c, Hanford Site Climatological Data Summary 1997, with Historical Data, PNNL-11794, Pacific Northwest Laboratory, Richland, Washington.

SNL, 1992, User's Guide for GENII-S: A Code for Statistical and Deterministic Simulations of Radiation Doses to Humans from Radionuclides in the Environment, SAND91-0561A, Sandia National Laboratories, Albuquerque, New Mexico.

WHC, 1988, Westinghouse Hanford Company Effluent Releases and Solid Waste Management Report for 1987: 200/600/1100 Areas, WHC-EP-0141, Westinghouse Hanford Company, Richland, Washington.

WHC, 1995a, Effluent Monitoring Quality Assurance Project Plan for Radioactive Airborne Emissions Data, WHC-EP-0528-2, Westinghouse Hanford Company, Richland, Washington. 
WHC, 1995b, Quality Assurance Program Plan for Radionuclide Airborne Emissions Monitoring, WHC-EP-0536-3, Westinghouse Hanford Company, Richland, Washington.

WMNW, 1998, Hanford Site Near-Facility Environmental Monitoring Annual Report, Calendar Year 1997, HNF-EP-0573-6, Waste Management Federal Services, Inc. Northwest Operations, Richland, Washington.

Zacks, R. S., March 1995, Biological Diversity-Inventory and Analysis at the Hanford Site: Insects, Department of Entomology, Washington State University, Pullman, Washington. 
DOE/RL-98-33

This page intentionally left blank. 
DOE/RL-98-33

APPENDIX

DOSE MODELING AND METEOROLOGICAL DATA 
DOE/RL-98-33

This page intentionally left blank. 
Figure A-1. Meteorological Station Map and Wind Roses for Calendar Year 1997.

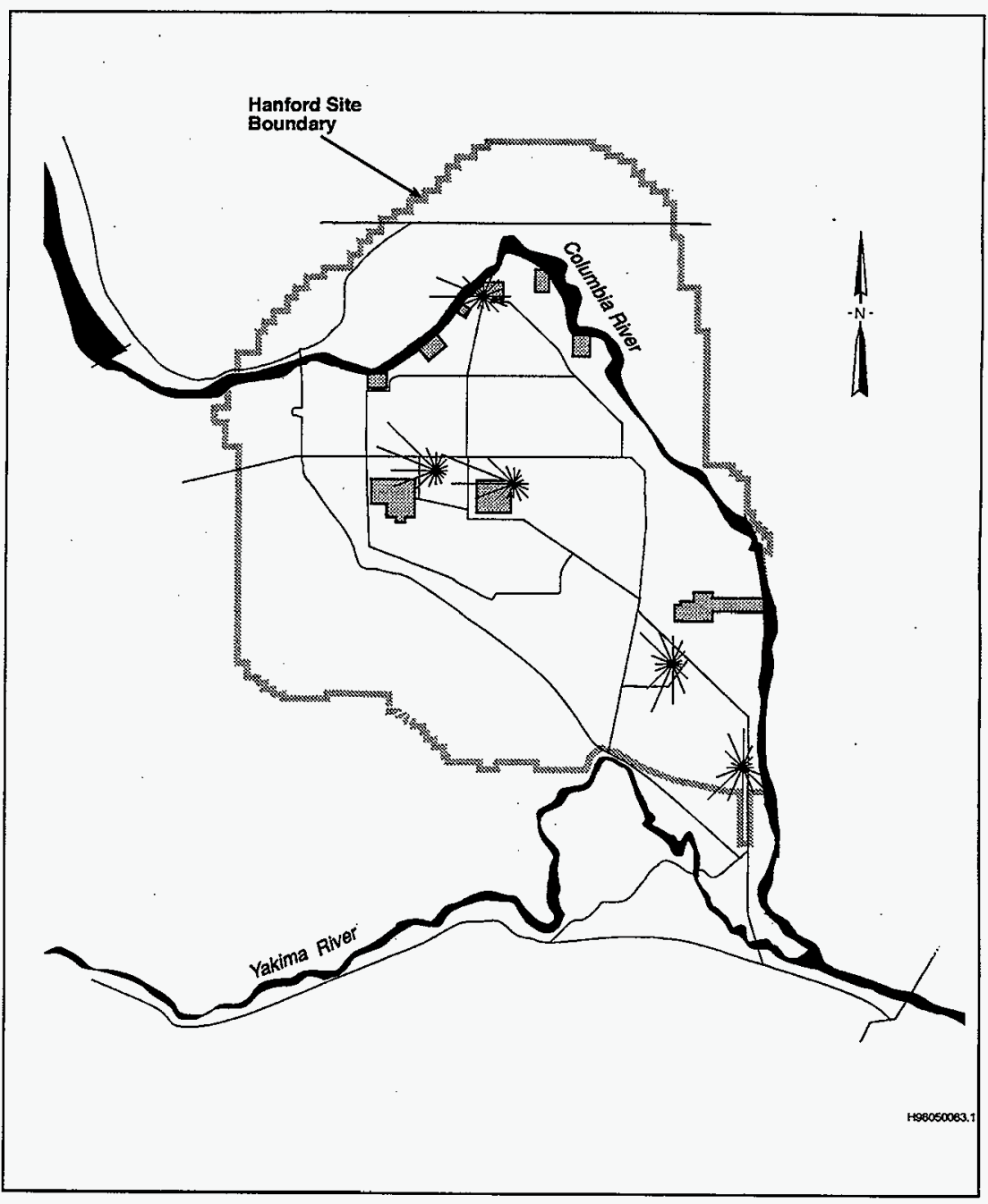


Figure A-2. 100 Area Wind Rose \& Histogram.

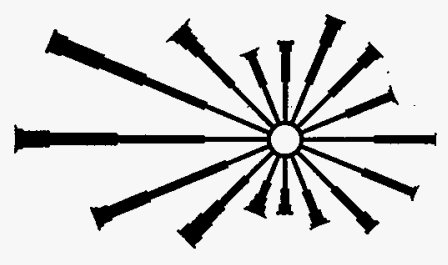

(a) Wind Rose

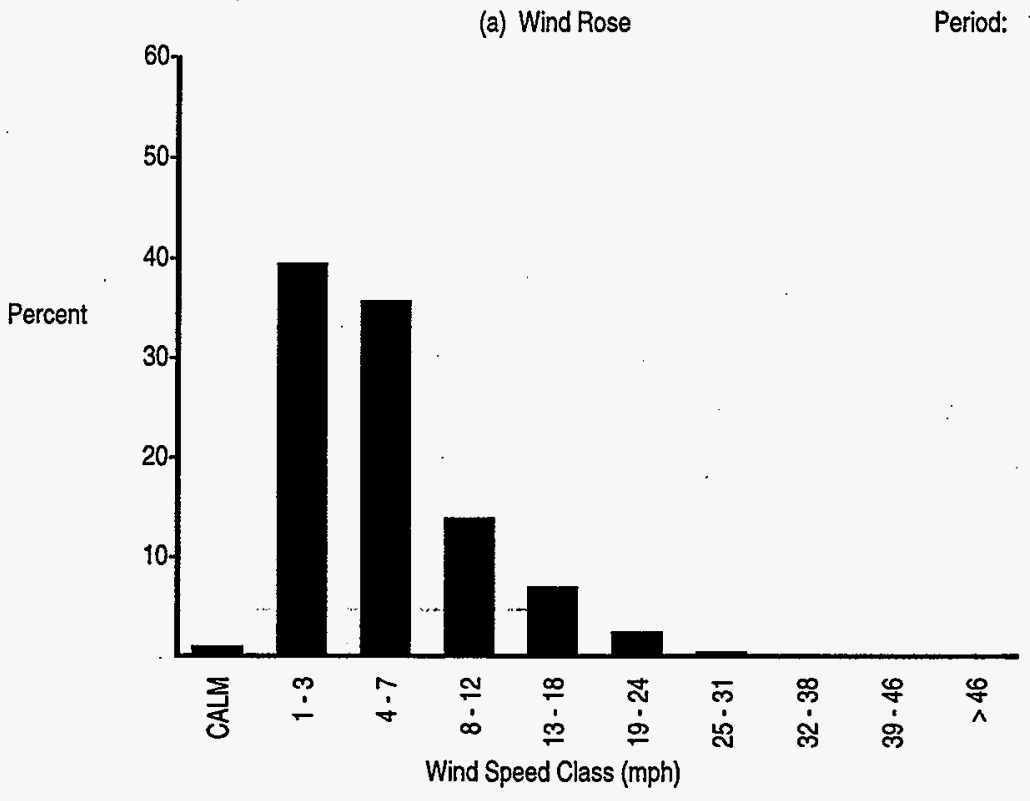

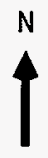

Station \#13 - 100N

(b) Wind Speed Histogram 
Figure A-3. 200 East Area Wind Rose \& Histogram.
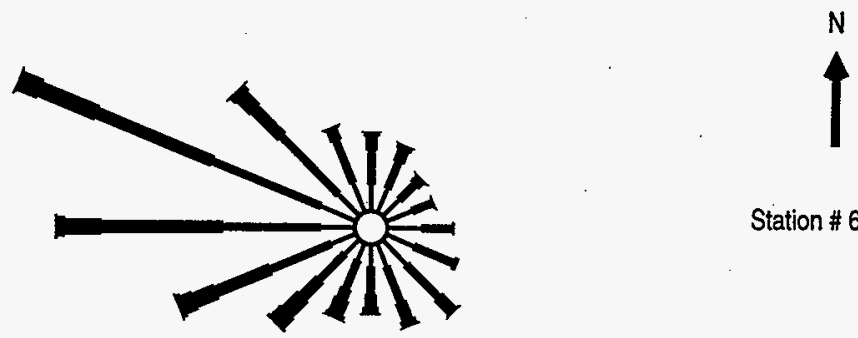

Station \# 6 - 200E

(a) Wind Rose

Period: $1 / 97-12 / 97$

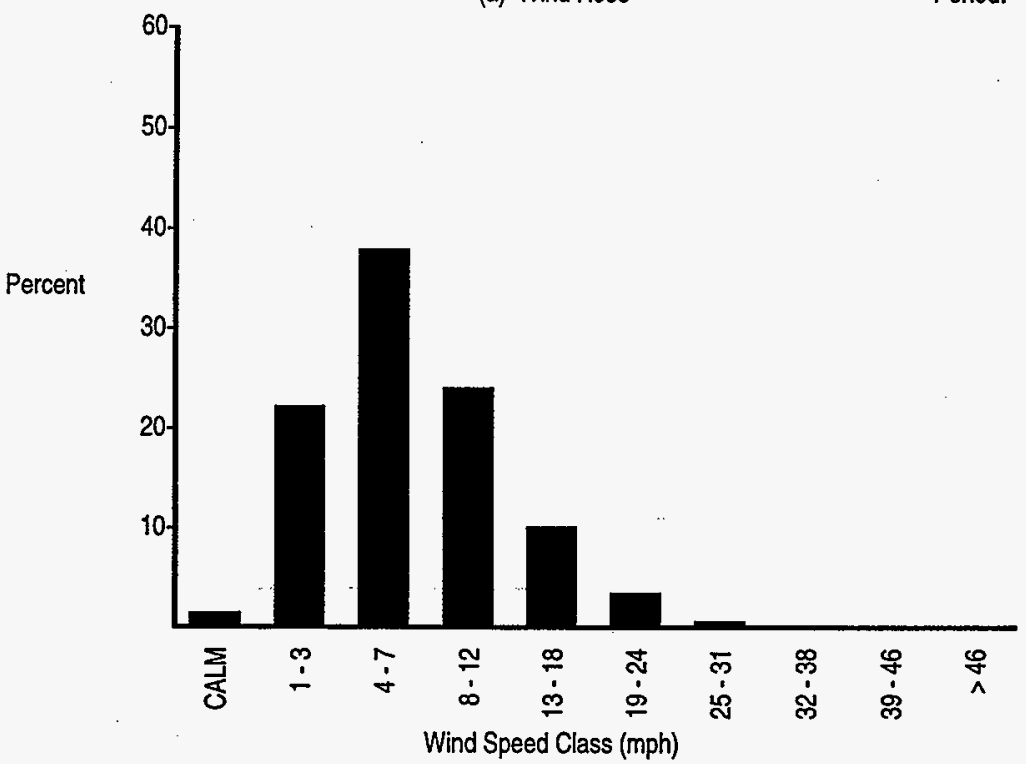

(b) Wind Speed Histogram 
Figure A-4. 200 West Area Wind Rose \& Histogram.
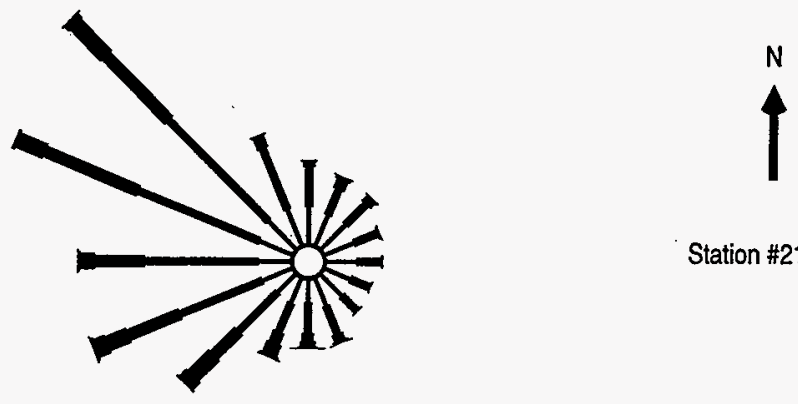

Station \#21 - HMS

(a) Wind Rose

Period: $1 / 97-12 / 97$

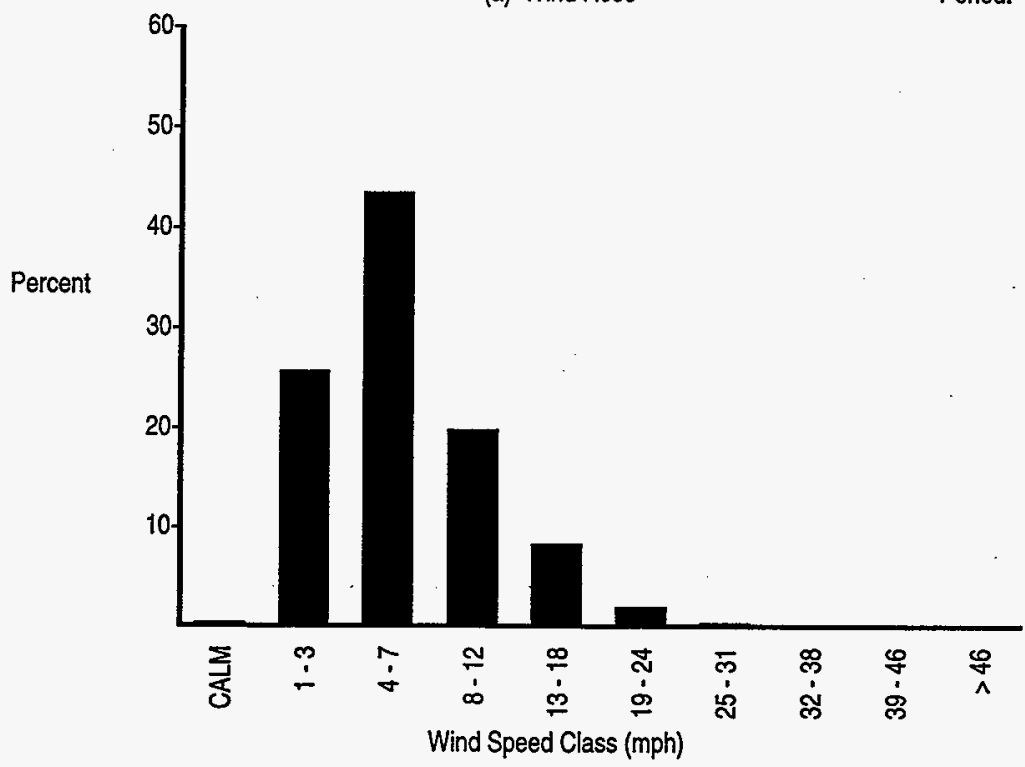

(b) Wind Speed Histogram 
Figure A-5. 300 Area Wind Rose \& Histogram.

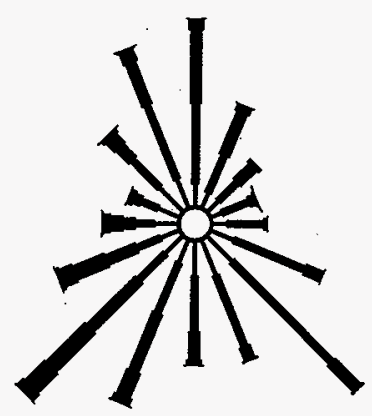

(a) Wind Rose

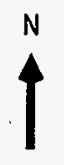

Station \#11 - 300A

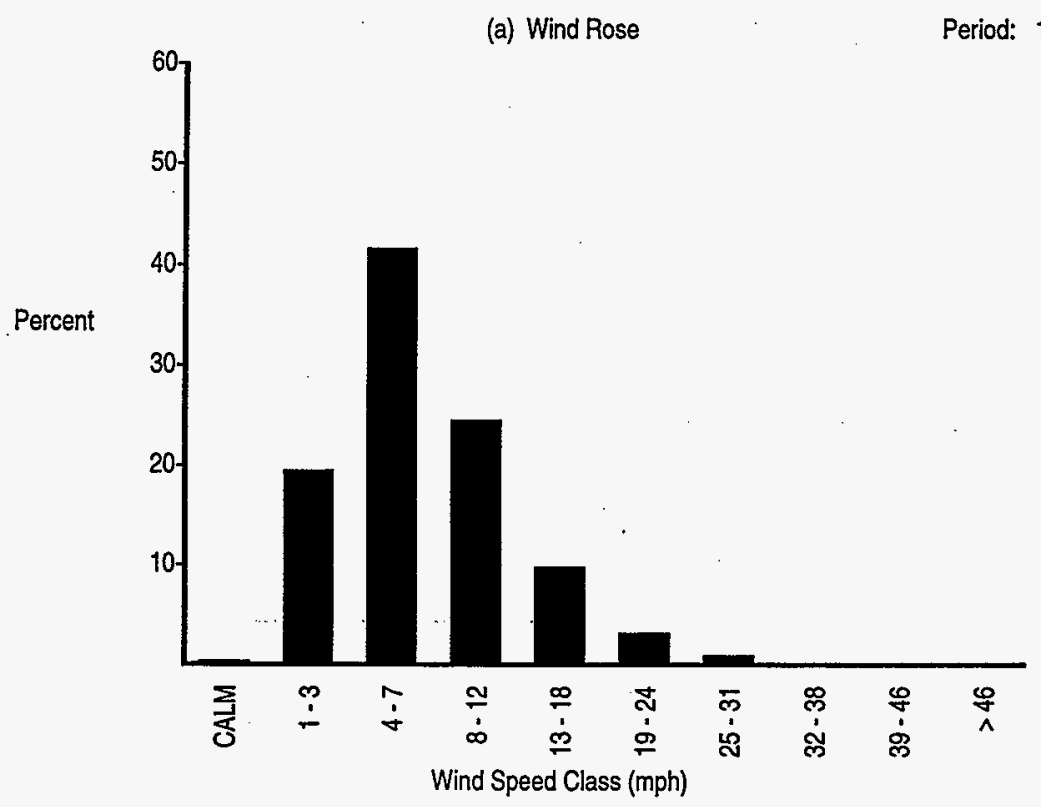

(b) Wind Speed Histogram 
Figure A-6. 400 Area Wind Rose \& Histogram.

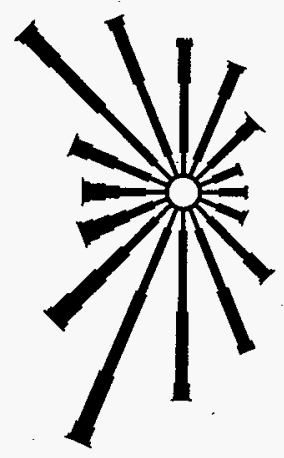

(a) Wind Rose

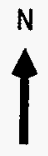

Station \# 9 - FFTF

Period: $1 / 97-12 / 97$

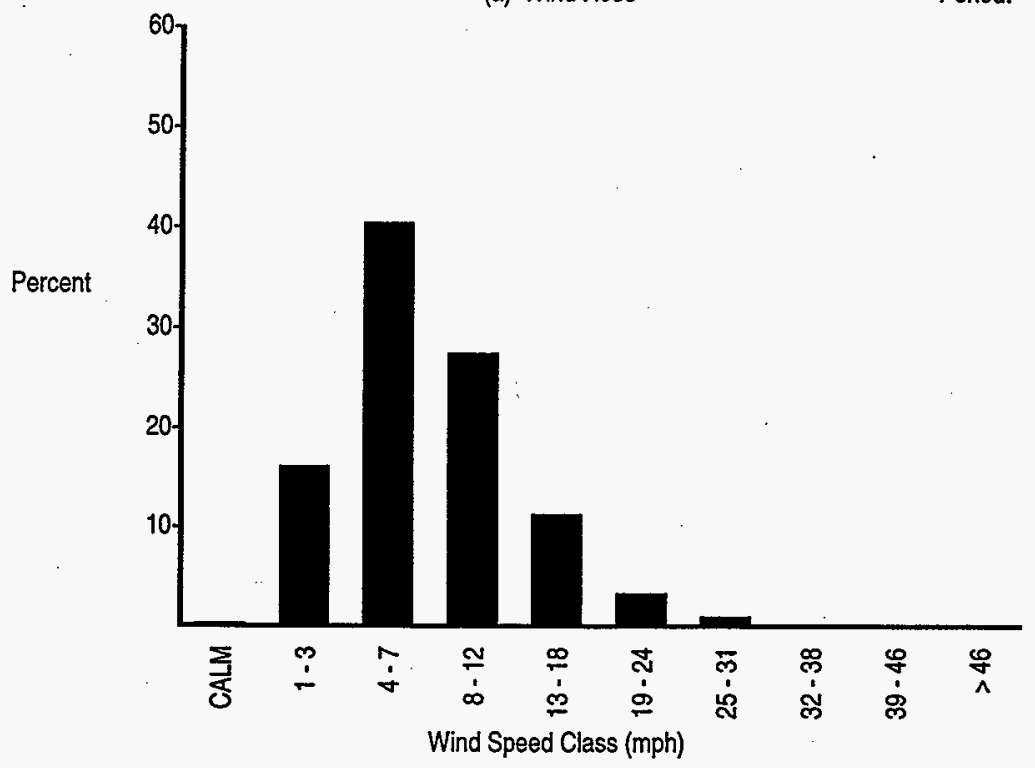

(b) Wind Speed Histogram 
Table A-1. Annual Average Dispersion Factor Around the 100 Area During 1997 for a $10-$ Meter Release Height.

\begin{tabular}{|c|c|c|c|c|c|c|c|c|c|c|}
\hline st: & $0.8 \mathrm{~km}$ & $2.4 \mathrm{~km}$ & $4.0 \mathrm{~km}$ & $5.6 \mathrm{~km}$ & $7.2 \mathrm{~km}$ & $12 \mathrm{~km}$ & $24 \mathrm{~km}$ & $40 \mathrm{~km}$ & $56 \mathrm{~km}$ & $72 \mathrm{~km}$ \\
\hline \multicolumn{11}{|l|}{ ir. } \\
\hline$\Gamma$ & $\begin{array}{l}2.21 \mathrm{E}-06 \\
2.14 \mathrm{E}-06 \\
3.16 \mathrm{E}-06 \\
4.89 \mathrm{E}-06\end{array}$ & $\begin{array}{l}4.26 \\
6.27 \\
1.00\end{array}$ & $\begin{array}{l}2.0 \\
2.0 \\
2.9 \\
4.7\end{array}$ & $\begin{array}{l}1.24 \\
1.82 \\
2.94\end{array}$ & $\begin{array}{l}8.6 \\
1.2 \\
2.0\end{array}$ & $\begin{array}{l}4.2 \\
6.2 \\
1.0\end{array}$ & $\begin{array}{l}1.6 \\
2.4 \\
3.9\end{array}$ & $\begin{array}{l}09 \\
09 \\
08 \\
08\end{array}$ & $\begin{array}{l}7 . \\
1 .\end{array}$ & $\begin{array}{l}3 . \\
5 . \\
9 .\end{array}$ \\
\hline & $\begin{array}{l}6.23 E-06 \\
5.89 E-06 \\
4.17 E-06 \\
2.62 E-06\end{array}$ & $\begin{array}{l}1.30 \mathrm{E}-06 \\
1.21 \mathrm{E}-06 \\
8.45 \mathrm{E}-07 \\
5.18 \mathrm{E}-07\end{array}$ & $\begin{array}{l}5.76 \mathrm{~B} \\
4.00 \mathrm{E} \\
2.45 \mathrm{E}\end{array}$ & $\begin{array}{l}3.56 \\
2.47 \\
1.51\end{array}$ & $\begin{array}{l}2.68 \mathrm{E}-07 \\
2.49 \mathrm{E}-07 \\
1.73 \mathrm{E}-07 \\
1.05 \mathrm{E}-07\end{array}$ & $\begin{array}{l}1.32 E-07 \\
1.23 E-07 \\
8.49 E-08 \\
5.18 E-08\end{array}$ & $\begin{array}{l}4.78 \\
3.3 \\
2.0\end{array}$ & $\begin{array}{l}2.4 \\
1.6 \\
1.0\end{array}$ & $\begin{array}{l}1.56 \\
1.56 \\
1.08 \\
6.59\end{array}$ & $\begin{array}{l}1.20 \mathrm{E}-08 \\
1.12 \mathrm{E}-08 \\
7.74 \mathrm{E}-09 \\
4.75 \mathrm{E}-09\end{array}$ \\
\hline & $\begin{array}{l}2.63 \\
3.18 \\
3.48 \\
4.07\end{array}$ & $\begin{array}{l}6.34 \mathrm{E} \\
6.90 \mathrm{E} \\
8.68 \mathrm{E}\end{array}$ & $\begin{array}{l}2.45 \\
3.00 \\
3.26 \\
4.16\end{array}$ & $\begin{array}{l}1.8 \\
2.0 \\
2.5\end{array}$ & $\begin{array}{l}1.0 \\
1.2 \\
1.4 \\
1.8\end{array}$ & $\begin{array}{l}6.3 \\
6.8 \\
9.0\end{array}$ & $\begin{array}{l}2.0 \\
2.4 \\
2.6 \\
3.5\end{array}$ & $\begin{array}{l}1.2 \\
1.3 \\
1.7\end{array}$ & & $\begin{array}{l}4.68 E-09 \\
5.80 E-09 \\
6.24 E-09 \\
8.33 E-09\end{array}$ \\
\hline N & $\begin{array}{l}5.06 \mathrm{E} \\
3.93 \mathrm{E} \\
2.73 \mathrm{E}\end{array}$ & $\begin{array}{l}1.17 \mathrm{E}-\mathrm{-} \\
1.02 \mathrm{E}-\mathrm{-} \\
7.81 \mathrm{E}-\mathrm{-} \\
5.44 \mathrm{E}-\mathrm{C}\end{array}$ & $\begin{array}{l}5.57 \mathrm{E}-07 \\
4.80 \mathrm{E}-07 \\
3.68 \mathrm{E}-07 \\
2.57 \mathrm{E}-07\end{array}$ & $\begin{array}{l}3.44 \mathrm{E}-07 \\
2.96 \mathrm{E}-07 \\
2.26 \mathrm{E}-07 \\
1.58 \mathrm{E}-07\end{array}$ & $\begin{array}{l}2.42 \mathrm{E}-07 \\
2.07 \mathrm{E}-07 \\
1.58 \mathrm{E}-07 \\
1.10 \mathrm{E}-07\end{array}$ & $\begin{array}{l}1.19 \\
1.01 \\
7.71 \\
5.38\end{array}$ & $\begin{array}{l}4.63 E-08 \\
3.91 E-08 \\
2.97 E-08 \\
2.08 E-08\end{array}$ & $\begin{array}{l}1.96 \mathrm{E}-08 \\
1.49 \mathrm{E}-08 \\
1.04 \mathrm{E}-08\end{array}$ & $\begin{array}{l}1.26 \mathrm{E}-08 \\
9.55 \mathrm{E}-09 \\
6.67 \mathrm{E}-09\end{array}$ & \\
\hline
\end{tabular}


Table A-2. Annual Average Dispersion Factor Around the 200 Areas During 1997 for an 89-Meter Release Height.

$\begin{array}{llllllllll}3.42 \mathrm{E}-08 & 2.53 \mathrm{E}-08 & 1.99 \mathrm{E}-08 & 1.56 \mathrm{E}-08 & 1.26 \mathrm{E}-08 & 7.80 \mathrm{E}-09 & 3.83 \mathrm{E}-09 & 2.22 \mathrm{E}-09 & 1.54 \mathrm{E}-09 & 1.17 \mathrm{E}-09 \\ 3.56 \mathrm{E}-08 & 2.60 \mathrm{E}-08 & 2.12 \mathrm{E}-08 & 1.69 \mathrm{E}-08 & 1.39 \mathrm{E}-08 & 8.76 \mathrm{E}-09 & 4.40 \mathrm{E}-09 & 2.58 \mathrm{E}-09 & 1.80 \mathrm{E}-09 & 1.37 \mathrm{E}-09 \\ 4.29 \mathrm{E}-08 & 3.48 \mathrm{E}-08 & 2.87 \mathrm{E}-08 & 2.30 \mathrm{E}-08 & 1.88 \mathrm{E}-08 & 1.18 \mathrm{E}-08 & 5.81 \mathrm{E}-09 & 3.35 \mathrm{E}-09 & 2.31 \mathrm{E}-09 & 1.74 \mathrm{E}-09 \\ 3.48 \mathrm{E}-08 & 3.73 \mathrm{E}-08 & 3.26 \mathrm{E}-08 & 2.66 \mathrm{E}-08 & 2.21 \mathrm{E}-08 & 1.42 \mathrm{E}-08 & 7.21 \mathrm{E}-09 & 4.24 \mathrm{E}-09 & 2.96 \mathrm{E}-09 & 2.25 \mathrm{E}-09 \\ 2.19 \mathrm{E}-08 & 3.34 \mathrm{E}-08 & 3.30 \mathrm{E}-08 & 2.88 \mathrm{E}-08 & 2.48 \mathrm{E}-08 & 1.69 \mathrm{E}-08 & 9.07 \mathrm{E}-09 & 5.49 \mathrm{E}-09 & 3.89 \mathrm{E}-09 & 2.99 \mathrm{E}-09 \\ 6.95 \mathrm{E}-08 & 7.46 \mathrm{E}-08 & 6.72 \mathrm{E}-08 & 5.58 \mathrm{E}-08 & 4.66 \mathrm{E}-08 & 3.01 \mathrm{E}-08 & 1.51 \mathrm{E}-08 & 8.80 \mathrm{E}-09 & 6.09 \mathrm{E}-09 & 4.61 \mathrm{E}-09 \\ 1.17 \mathrm{E}-07 & 9.74 \mathrm{E}-08 & 7.73 \mathrm{E}-08 & 6.06 \mathrm{E}-08 & 4.92 \mathrm{E}-08 & 3.05 \mathrm{E}-08 & 1.50 \mathrm{E}-08 & 8.60 \mathrm{E}-09 & 5.93 \mathrm{E}-09 & 4.47 \mathrm{E}-09 \\ 9.68 \mathrm{E}-08 & 6.77 \mathrm{E}-08 & 5.22 \mathrm{E}-08 & 4.01 \mathrm{E}-08 & 3.21 \mathrm{E}-08 & 1.94 \mathrm{E}-08 & 9.16 \mathrm{E}-09 & 5.15 \mathrm{E}-09 & 3.51 \mathrm{E}-09 & 2.62 \mathrm{E}-09 \\ 5.75 \mathrm{E}-08 & 3.41 \mathrm{E}-08 & 2.58 \mathrm{E}-08 & 1.99 \mathrm{E}-08 & 1.59 \mathrm{E}-08 & 9.73 \mathrm{E}-09 & 4.68 \mathrm{E}-09 & 2.66 \mathrm{E}-09 & 1.82 \mathrm{E}-09 & 1.37 \mathrm{E}-09 \\ 8.70 \mathrm{E}-08 & 4.73 \mathrm{E}-08 & 3.49 \mathrm{E}-08 & 2.64 \mathrm{E}-08 & 2.08 \mathrm{E}-08 & 1.22 \mathrm{E}-08 & 5.64 \mathrm{E}-09 & 3.13 \mathrm{E}-09 & 2.12 \mathrm{E}-09 & 1.58 \mathrm{E}-09 \\ 8.02 \mathrm{E}-08 & 4.24 \mathrm{E}-08 & 3.02 \mathrm{E}-08 & 2.26 \mathrm{E}-08 & 1.79 \mathrm{E}-08 & 1.07 \mathrm{E}-08 & 5.05 \mathrm{E}-09 & 2.85 \mathrm{E}-09 & 1.95 \mathrm{E}-09 & 1.46 \mathrm{E}-09 \\ 7.72 \mathrm{E}-08 & 4.67 \mathrm{E}-08 & 3.40 \mathrm{E}-08 & 2.54 \mathrm{E}-08 & 2.00 \mathrm{E}-08 & 1.17 \mathrm{E}-08 & 5.39 \mathrm{E}-09 & 2.99 \mathrm{E}-09 & 2.02 \mathrm{E}-09 & 1.50 \mathrm{E}-09 \\ 6.30 \mathrm{E}-08 & 4.18 \mathrm{E}-08 & 3.29 \mathrm{E}-08 & 2.53 \mathrm{E}-08 & 2.02 \mathrm{E}-08 & 1.20 \mathrm{E}-08 & 5.55 \mathrm{E}-09 & 3.07 \mathrm{E}-09 & 2.07 \mathrm{E}-09 & 1.54 \mathrm{E}-09 \\ 4.77 \mathrm{E}-09 & 9.24 \mathrm{E}-09 & 8.36 \mathrm{E}-09 & 6.74 \mathrm{E}-09 & 5.47 \mathrm{E}-09 & 3.32 \mathrm{E}-09 & 1.55 \mathrm{E}-09 & 8.64 \mathrm{E}-10 & 5.87 \mathrm{E}-10 & 4.38 \mathrm{E}-10 \\ 6.59 \mathrm{E}-08 & 5.42 \mathrm{E}-08 & 4.37 \mathrm{E}-08 & 3.45 \mathrm{E}-08 & 2.80 \mathrm{E}-08 & 1.73 \mathrm{E}-08 & 8.34 \mathrm{E}-09 & 4.72 \mathrm{E}-09 & 3.22 \mathrm{E}-09 & 2.41 \mathrm{E}-09 \\ 3.92 \mathrm{E}-08 & 3.27 \mathrm{E}-08 & 2.68 \mathrm{E}-08 & 2.15 \mathrm{E}-08 & 1.78 \mathrm{E}-08 & 1.14 \mathrm{E}-08 & 5.75 \mathrm{E}-09 & 3.37 \mathrm{E}-09 & 2.35 \mathrm{E}-09 & 1.78 \mathrm{E}-09\end{array}$

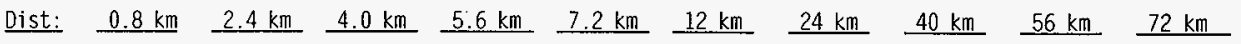

Dir:

N
NNE
ENE

SSW

WW

W

WWW

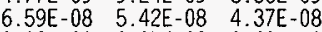
$3.92 \mathrm{E}-08$

$2.15 \mathrm{E}-08$

$\begin{array}{ll}2.80 \mathrm{E}-08 & 1.73 \mathrm{E}-08 \\ 1.78 \mathrm{E}-08 & 1.14 \mathrm{E}-08\end{array}$

.34E-09

$\begin{array}{lll}4.37 \mathrm{E}-09 & 3.22 \mathrm{E}-09 & 2.41 \mathrm{E}-09 \\ 2.35 \mathrm{E}-09 & 1.78 \mathrm{E}-09\end{array}$ 
Table A-3. Annual Average Dispersion Factor Around the 300 Area During 1997 for a 10-Meter Release Height.

Dist: $\quad 0.8 \mathrm{~km} \quad 2.4 \mathrm{~km} \quad 4.0 \mathrm{~km} \quad \underline{5.6 \mathrm{~km}} \quad \underline{7.2 \mathrm{~km}} \quad \underline{12 \cdot \mathrm{km}} \quad \underline{24 \mathrm{~km}} \quad \underline{40 \mathrm{~km}} \quad \underline{56 \mathrm{~km}} \quad \underline{72 \mathrm{~km}}$ Dir:

\begin{tabular}{|c|c|c|c|c|c|c|c|c|c|}
\hline $\begin{array}{l}3.78 \mathrm{E}-06 \\
3.20 \mathrm{E}-06 \\
3.12 \mathrm{E}-06 \\
2.21 \mathrm{E}-06\end{array}$ & $\begin{array}{l}7.75 E-07 \\
6.21 E-07 \\
5.98 E-07 \\
4.33 E-07\end{array}$ & $\begin{array}{l}3.68 \mathrm{E}-07 \\
2.92 \mathrm{E}-07 \\
2.80 \mathrm{E}-07 \\
2.04 \mathrm{E}-07\end{array}$ & $\begin{array}{l}2.28 \mathrm{E}-07 \\
1.79 \mathrm{E}-07 \\
1.72 \mathrm{E}-07 \\
1.25 \mathrm{E}-07\end{array}$ & $\begin{array}{l}1.60 E-07 \\
1.25 E-07 \\
1.20 E-07 \\
8.72 E-08\end{array}$ & $\begin{array}{l}7.85 E-08 \\
6.10 E-08 \\
5.86 E-08 \\
4.25 E-08\end{array}$ & $\begin{array}{l}3.05 \mathrm{E}-08 \\
2.36 \mathrm{E}-08 \\
2.27 \mathrm{E}-08 \\
1.64 \mathrm{E}-08\end{array}$ & $\begin{array}{l}1.54 \mathrm{E}-08 \\
1.19 \mathrm{E}-08 \\
1.14 \mathrm{E}-08 \\
8.19 \mathrm{E}-09\end{array}$ & $\begin{array}{l}9.88 E-09 \\
7.59 E-09 \\
7.30 E-09 \\
5.23 E-09\end{array}$ & $\begin{array}{l}7.11 \mathrm{E}-09 \\
5.45 \mathrm{E}-09 \\
5.24 \mathrm{E}-09 \\
3.75 \mathrm{E}-09\end{array}$ \\
\hline $\begin{array}{l}1.99 E-06 \\
2.03 E-06 \\
3.37 E-06 \\
4.16 E-06\end{array}$ & $\begin{array}{l}3.93 \mathrm{E}-07 \\
4.28 \mathrm{E}-07 \\
7.05 \mathrm{E}-07 \\
8.66 \mathrm{E}-07\end{array}$ & $\begin{array}{l}1.85 \mathrm{E}-07 \\
2.04 \mathrm{E}-07 \\
3.36 \mathrm{E}-07 \\
4.12 \mathrm{E}-07\end{array}$ & $\begin{array}{l}1.14 E-07 \\
1.26 E-07 \\
2.08 E-07 \\
2.54 E-07\end{array}$ & $\begin{array}{l}7.94 \mathrm{E}-08 \\
8.85 \mathrm{E}-08 \\
1.46 \mathrm{E}-07 \\
1.78 \mathrm{E}-07\end{array}$ & $\begin{array}{l}3.87 \mathrm{E}-08 \\
4.34 \mathrm{E}-08 \\
7.17 \mathrm{E}-08 \\
8.73 \mathrm{E}-08\end{array}$ & $\begin{array}{l}1.49 \mathrm{E}-08 \\
1.69 \mathrm{E}-08 \\
2.79 \mathrm{E}-08 \\
3.39 \mathrm{E}-08\end{array}$ & $\begin{array}{l}7.47 \mathrm{E}-09 \\
8.49 \mathrm{E}-09 \\
1.41 \mathrm{E}-08 \\
1.71 \mathrm{E}-08\end{array}$ & $\begin{array}{l}.09 \\
.09 \\
09 \\
.08\end{array}$ & $\begin{array}{l}3.91 \mathrm{E}-09 \\
6.49 \mathrm{E}-09 \\
7.85 \mathrm{E}-09\end{array}$ \\
\hline $\begin{array}{l}3.73 E-06 \\
1.92 E-06 \\
1.16 E-06 \\
8.74 E-07\end{array}$ & $\begin{array}{l}7.50 \mathrm{E}-07 \\
3.78 \mathrm{E}-07 \\
2.19 \mathrm{E}-07 \\
1.60 \mathrm{E}-07\end{array}$ & $\begin{array}{l}3.54 \mathrm{E}-07 \\
1.78 \mathrm{E}-07 \\
1.02 \mathrm{E}-07 \\
7.42 \mathrm{E}-08\end{array}$ & $\begin{array}{l}2.18 \mathrm{E}-07 \\
1.09 \mathrm{E}-07 \\
6.21 \mathrm{E}-08 \\
4.52 \mathrm{E}-08\end{array}$ & $\begin{array}{l}1.53 \mathrm{E}-07 \\
7.62 \mathrm{E}-08 \\
4.32 \mathrm{E}-08 \\
3.14 \mathrm{E}-08\end{array}$ & $\begin{array}{l}7.46 \mathrm{E}-08 \\
3.72 \mathrm{E}-08 \\
2.10 \mathrm{E}-08 \\
1.52 \mathrm{E}-08\end{array}$ & $\begin{array}{l}2.88 \mathrm{E}-08 \\
1.44 \mathrm{E}-08 \\
8.10 \mathrm{E}-09 \\
5.86 \mathrm{E}-09\end{array}$ & $\begin{array}{l}1.45 E-08 \\
7.23 E-09 \\
4.08 E-09 \\
2.95 E-09\end{array}$ & $\begin{array}{l}9.26 \mathrm{E}-09 \\
4.64 \mathrm{E}-09 \\
2.62 \mathrm{E}-09 \\
1.90 \mathrm{E}-09\end{array}$ & $\begin{array}{l}6.64 \mathrm{E}-09 \\
3.33 \mathrm{E}-09 \\
1.88 \mathrm{E}-09 \\
1.36 \mathrm{E}-09\end{array}$ \\
\hline $\begin{array}{l}1.16 E-06 \\
2.98 E-06 \\
5.42 E-06 \\
4.21 E-06\end{array}$ & $\begin{array}{l}2.11 \mathrm{E}-07 \\
6.02 \mathrm{E}-07 \\
1.12 \mathrm{E}-06 \\
8.85 \mathrm{E}-07\end{array}$ & $\begin{array}{l}9.79 \mathrm{E}-08 \\
2.86 \mathrm{E}-07 \\
5.35 \mathrm{E}-07 \\
4.24 \mathrm{E}-07\end{array}$ & $\begin{array}{l}5.98 \mathrm{E}-08 \\
1.76 \mathrm{E}-07 \\
3.31 \mathrm{E}-07 \\
2.63 \mathrm{E}-07\end{array}$ & $\begin{array}{l}4.17 \mathrm{E}-08 \\
1.24 \mathrm{E}-07 \\
2.32 \mathrm{E}-07 \\
1.85 \mathrm{E}-07\end{array}$ & $\begin{array}{l}2.03 \mathrm{E}-08 \\
6.08 \mathrm{E}-08 \\
1.15 \mathrm{E}-07 \\
9.12 \mathrm{E}-08\end{array}$ & $\begin{array}{l}7.88 \\
2.37 \\
4.47 \\
3.57\end{array}$ & & $\begin{array}{l}.09 \\
.09 \\
.08 \\
.08\end{array}$ & \\
\hline
\end{tabular}


Table A-4. Annual Average Dispersion Factor Around the 400 Area During 1997 for a 10-Meter Release Height.

Dist: $\quad \underline{0.8 \mathrm{~km}} \quad \underline{2.4 \mathrm{~km}} \quad \underline{4.0 \mathrm{~km}} \quad \underline{5.6 \mathrm{~km}} \quad \underline{7.2 \mathrm{~km}} \quad 12 \mathrm{~km} \quad 24 \mathrm{~km} \quad 40 \mathrm{~km} \quad 56 \quad \underline{\mathrm{km}} \quad \underline{72 \mathrm{~km}}$

Dir:

N $\quad 3.78 E-06 \quad 7.69 \mathrm{E}-07 \quad 3.65 \mathrm{E}-07 \quad 2.26 \mathrm{E}-07 \quad 1.58 \mathrm{E}-07 \quad 7.79 \mathrm{E}-08 \quad 3.04 \mathrm{E}-08 \quad 1.54 \mathrm{E}-08 \quad 9.88 \mathrm{E}-09 \quad 7.11 \mathrm{E}-09$

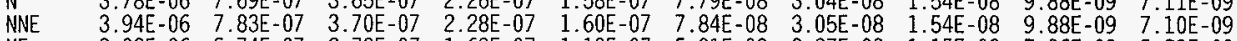

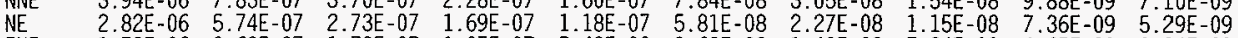

$\underset{⿱}{\stackrel{1}{N}}$

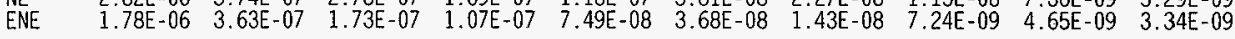

E $\quad \begin{array}{llllllllll}1.98 \mathrm{E}-06 & 3.93 \mathrm{E}-07 & 1.85 \mathrm{E}-07 & 1.14 \mathrm{E}-07 & 7.98 \mathrm{E}-08 & 3.90 \mathrm{E}-08 & 1.51 \mathrm{E}-08 & 7.61 \mathrm{E}-09 & 4.88 \mathrm{E}-09 & 3.50 \mathrm{E}-09\end{array}$

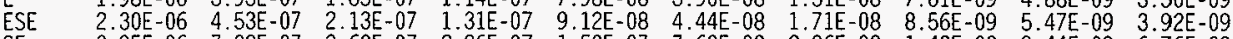
$\begin{array}{lllllllllll}\mathrm{SE} & 3.95 \mathrm{E}-06 & 7.82 \mathrm{E}-07 & 3.68 \mathrm{E}-07 & 2.26 \mathrm{E}-07 & 1.58 \mathrm{E}-07 & 7.68 \mathrm{E}-08 & 2.96 \mathrm{E}-08 & 1.48 \mathrm{E}-08 & 9.44 \mathrm{E}-09 & 6.76 \mathrm{E}-09\end{array}$

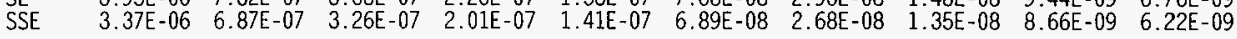

$\begin{array}{lllllllllll}\mathrm{S} & 2.77 \mathrm{E}-06 & 5.72 \mathrm{E}-07 & 2.72 \mathrm{E}-07 & 1.68 \mathrm{E}-07 & 1.18 \mathrm{E}-07 & 5.78 \mathrm{E}-08 & 2.25 \mathrm{E}-08 & 1.14 \mathrm{E}-08 & 7.30 \mathrm{E}-09 & 5.26 \mathrm{E}-09\end{array}$

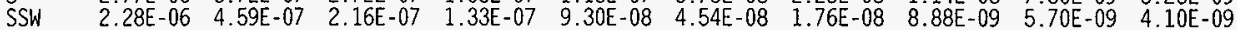

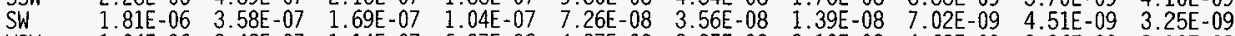

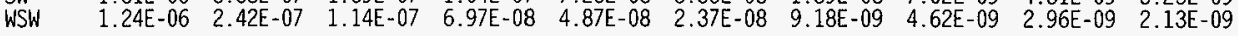

W $\quad 1.30 \mathrm{E}-06 \quad 2.51 \mathrm{E}-07 \quad 1.18 \mathrm{E}-07 \quad 7.25 \mathrm{E}-08 \quad 5.07 \mathrm{E}-08 \quad 2.47 \mathrm{E}-08 \quad 9.58 \mathrm{E}-09 \quad 4.82 \mathrm{E}-09 \quad 3.09 \mathrm{E}-09 \quad 2.22 \mathrm{E}-09$

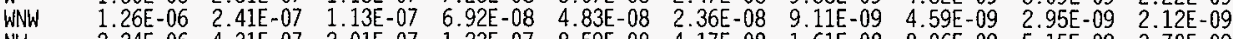

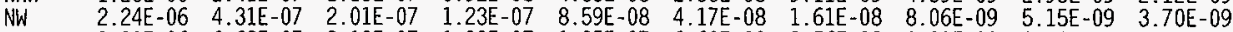

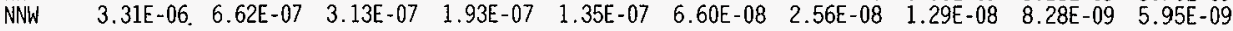


DOE/RL-98-33

Table A-5. Radionuclide Data Used for CAP88-PC Dose Calculations at Hanford.

\begin{tabular}{lcccc} 
Nuclide & $\begin{array}{c}\text { (1 of } 3 \text { sheets) } \\
\text { Clearance } \\
\text { Class }\end{array}$ & $\begin{array}{c}\text { Particle } \\
\text { Size } \\
\text { (microns) }\end{array}$ & $\begin{array}{c}\text { Scavenging } \\
\text { Coefficient } \\
\text { (Der second) }\end{array}$ & $\begin{array}{c}\text { Dry } \\
\text { Deposition } \\
\text { Velocity } \\
\text { (m/s) }\end{array}$ \\
\hline H-3 & $*$ & 0.0 & $0.00 \mathrm{E}+00$ & $0.00 \mathrm{E}+00$ \\
C0-60 & $Y$ & 1.0 & $1.60 \mathrm{E}-06$ & $1.80 \mathrm{E}-03$ \\
SR-90 & $D$ & 1.0 & $1.60 \mathrm{E}-06$ & $1.80 \mathrm{E}-03$ \\
SB-125 & $W$ & 1.0 & $1.60 \mathrm{E}-06$ & $1.80 \mathrm{E}-03$ \\
I-129 & 0 & 1.0 & $1.60 \mathrm{E}-06$ & $3.50 \mathrm{E}-02$ \\
CS-137 & 0 & 1.0 & $1.60 \mathrm{E}-06$ & $1.80 \mathrm{E}-03$ \\
BA-137M & 0 & 1.0 & $1.60 \mathrm{E}-06$ & $1.80 \mathrm{E}-03$ \\
PU-238 & $Y$ & 1.0 & $1.60 \mathrm{E}-06$ & $1.80 \mathrm{E}-03$ \\
PU-239 & $Y$ & 1.0 & $1.60 \mathrm{E}-06$ & $1.80 \mathrm{E}-03$ \\
PU-241 & $Y$ & 1.0 & $1.60 \mathrm{E}-06$ & $1.80 \mathrm{E}-03$ \\
AM-241 & W & 1.0 & $1.60 \mathrm{E}-06$ & $1.80 \mathrm{E}-03$ \\
PB-212 & $\mathrm{D}$ & 1.0 & $1.60 \mathrm{E}-06$ & $1.80 \mathrm{E}-03$ \\
RN-222 & $*$ & 0.0 & $0.00 \mathrm{E}+00$ & $0.00 \mathrm{E}+00$
\end{tabular}


Table A-5. Radionuclide Data Used for CAP88-PC Dose Calculations at Hanford.

(2 of 3 sheets)

\begin{tabular}{|c|c|c|c|c|c|}
\hline \multirow[b]{2}{*}{ Nuclide } & \multicolumn{3}{|c|}{ DECAY CONSTANT (PER DAY) } & \multicolumn{2}{|c|}{ TRANSFER COEFFICIENT } \\
\hline & $\begin{array}{l}\text { Radio- } \\
\text { active (1) }\end{array}$ & Surface & Water & Milk (2) & Meat (3) \\
\hline $\begin{array}{l}H-3 \\
C 0-60 \\
\text { SR-90 } \\
\text { RU-106 } \\
\text { SN-113 } \\
\text { SB-125 } \\
I-129 \\
\text { CS-134 } \\
\text { CS }-137 \\
\text { BA-137M } \\
\text { PU-238 } \\
\text { PU-239 } \\
\text { PU-241 } \\
\text { AM-241 }\end{array}$ & $\begin{array}{l}0.00 \mathrm{E}+00 \\
0.00 \mathrm{E}+00 \\
0.00 \mathrm{E}+00 \\
0.00 \mathrm{E}+00 \\
0.00 \mathrm{E}+00 \\
0.00 \mathrm{E}+00 \\
0.00 \mathrm{E}+00 \\
0.00 \mathrm{E}+00 \\
0.00 \mathrm{E}+00 \\
3.91 \mathrm{E}+02 \\
0.00 \mathrm{E}+00 \\
0.00 \mathrm{E}+00 \\
0.00 \mathrm{E}+00 \\
0.00 \mathrm{E}+00\end{array}$ & $\begin{array}{l}5.48 \mathrm{E}-05 \\
5.48 \mathrm{E}-05 \\
5.48 \mathrm{E}-05 \\
5.48 \mathrm{E}-05 \\
5.48 \mathrm{E}-05 \\
5.48 \mathrm{E}-05 \\
5.48 \mathrm{E}-05 \\
5.48 \mathrm{E}-05 \\
5.48 \mathrm{E}-05 \\
5.48 \mathrm{E}-05 \\
5.48 \mathrm{E}-05 \\
5.48 \mathrm{E}-05 \\
5.48 \mathrm{E}-05 \\
5.48 \mathrm{E}-05\end{array}$ & $\begin{array}{l}0.00 \mathrm{E}+00 \\
0.00 \mathrm{E}+00 \\
0.00 \mathrm{E}+00 \\
0.00 \mathrm{E}+00 \\
0.00 \mathrm{E}+00 \\
0.00 \mathrm{E}+00 \\
0.00 \mathrm{E}+00 \\
0.00 \mathrm{E}+00 \\
0.00 \mathrm{E}+00 \\
0.00 \mathrm{E}+00 \\
0.00 \mathrm{E}+00 \\
0.00 \mathrm{E}+00 \\
0.00 \mathrm{E}+00 \\
0.00 \mathrm{E}+00\end{array}$ & $\begin{array}{l}0.00 \mathrm{E}+00 \\
2.00 \mathrm{E}-03 \\
1.50 \mathrm{E}-03 \\
6.00 \mathrm{E}-07 \\
1.00 \mathrm{E}-03 \\
1.00 \mathrm{E}-04 \\
1.00 \mathrm{E}-02 \\
7.00 \mathrm{E}-03 \\
7.00 \mathrm{E}-03 \\
3.50 \mathrm{E}-04 \\
1.00 \mathrm{E}-07 \\
1.00 \mathrm{E}-07 \\
1.00 \mathrm{E}-07 \\
4.00 \mathrm{E}-07\end{array}$ & $\begin{array}{l}0.00 \mathrm{E}+00 \\
2.00 \mathrm{E}-02 \\
3.00 \mathrm{E}-04 \\
2.00 \mathrm{E}-03 \\
8.00 \mathrm{E}-02 \\
1.00 \mathrm{E}-03 \\
7.00 \mathrm{E}-03 \\
2.00 \mathrm{E}-02 \\
2.00 \mathrm{E}-02 \\
1.50 \mathrm{E}-04 \\
5.00 \mathrm{E}-07 \\
5.00 \mathrm{E}-07 \\
5.00 \mathrm{E}-07 \\
3.50 \mathrm{E}-06\end{array}$ \\
\hline $\begin{array}{l}P B-212 \\
R N-222\end{array}$ & $\begin{array}{l}1.56 \mathrm{E}+00 \\
1.81 \mathrm{E}-01\end{array}$ & $\begin{array}{l}5.48 E-05 \\
5.48 E-05\end{array}$ & $\begin{array}{l}0.00 E+00 \\
0.00 E+00\end{array}$ & $\begin{array}{l}2.50 E-04 \\
0.00 E+00\end{array}$ & $\begin{array}{l}3.00 \mathrm{E}-04 \\
0.00 \mathrm{E}+00\end{array}$ \\
\hline
\end{tabular}

FOOTNOTES: (1) Effective radioactive decay constant in plume; set to zero if less than $1.0 \mathrm{E}-2$

(2) Fraction of animal's daily intake of nuclide which appears in each $\mathrm{L}$ of milk (days $/ \mathrm{L}$ )

(3) Fraction of animal's daily intake of nuclide which appears in each $\mathrm{kg}$ of meat (days $/ \mathrm{kg}$ ) 
Table A-5. Radionuclide Data Used for CAP88-PC Dose Calculations at Hanford.

( 3 of 3 sheets)

\begin{tabular}{|c|c|c|c|c|}
\hline \multirow[b]{2}{*}{ Nuclide } & \multicolumn{2}{|c|}{$\begin{array}{l}\text { CONCENTRATION } \\
\text { UPTAKE FACTOR }\end{array}$} & \multicolumn{2}{|c|}{ GI UPTAKE FRACTION } \\
\hline & Forage (1) & Edible (2) & Inhalation & Ingestion \\
\hline $\begin{array}{l}H-3 \\
C O-60 \\
\text { SR-90 } \\
I-129 \\
\text { SB-125 } \\
\text { CS-137 } \\
\text { BA-137M } \\
\text { PU-238 } \\
\text { PU-239 } \\
\text { PU-241 } \\
\text { AM-241 }\end{array}$ & $\begin{array}{l}0.00 \mathrm{E}+00 \\
2.00 \mathrm{E}-02 \\
2.50 \mathrm{E}+00 \\
1.50 \mathrm{E}-01 \\
2.00 \mathrm{E}-01 \\
8.00 \mathrm{E}-02 \\
1.50 \mathrm{E}-01 \\
4.50 \mathrm{E}-04 \\
4.50 \mathrm{E}-04 \\
4.50 \mathrm{E}-04 \\
5.50 \mathrm{E}-03\end{array}$ & $\begin{array}{l}0.00 \mathrm{E}+00 \\
3.00 \mathrm{E}-03 \\
1.07 \mathrm{E}-01 \\
2.14 \mathrm{E}-02 \\
1.28 \mathrm{E}-02 \\
1.28 \mathrm{E}-02 \\
6.42 \mathrm{E}-03 \\
1.93 \mathrm{E}-05 \\
1.93 \mathrm{E}-05 \\
1.93 \mathrm{E}-05 \\
1.07 \mathrm{E}-04\end{array}$ & $\begin{array}{l}9.50 \mathrm{E}-01 \\
5.00 \mathrm{E}-02 \\
3.00 \mathrm{E}-01 \\
9.50 \mathrm{E}-01 \\
1.00 \mathrm{E}-02 \\
9.50 \mathrm{E}-01 \\
1.00 \mathrm{E}-01 \\
1.00 \mathrm{E}-03 \\
1.00 \mathrm{E}-04 \\
1.00 \mathrm{E}-03 \\
1.00 \mathrm{E}-03\end{array}$ & $\begin{array}{l}9.50 \mathrm{E}-01 \\
3.00 \mathrm{E}-01 \\
3.00 \mathrm{E}-01 \\
9.50 \mathrm{E}-01 \\
1.00 \mathrm{E}-01 \\
9.50 \mathrm{E}-01 \\
1.00 \mathrm{E}-01 \\
1.00 \mathrm{E}-03 \\
1.00 \mathrm{E}-03 \\
1.00 \mathrm{E}-03 \\
1.00 \mathrm{E}-03\end{array}$ \\
\hline $\begin{array}{l}P B-212 \\
R N-222\end{array}$ & $\begin{array}{l}4.50 \mathrm{E}-02 \\
0.00 \mathrm{E}+00\end{array}$ & $\begin{array}{l}3.85 \mathrm{E}-03 \\
0.00 \mathrm{E}+00\end{array}$ & $\begin{array}{l}2.00 \mathrm{E}-01 \\
0.00 \mathrm{E}+00\end{array}$ & $\begin{array}{l}2.00 \mathrm{E}-01 \\
0.00 \mathrm{E}+00\end{array}$ \\
\hline FOOTNOTES: & \multicolumn{4}{|c|}{$\begin{array}{l}\text { (1) Concentration factor for uptake of nuclide from soil for } \\
\text { pasture and forage (in } \mathrm{pCi} / \mathrm{kg} \text { dry weight per } \mathrm{pCi} / \mathrm{kg} \text { dry } \\
\text { soil) }\end{array}$} \\
\hline
\end{tabular}


Table A-6. Exposure and Consumption Data for the Hanford Site.

( 1 of 2 sheets)

FOOD SOURCE FOR THE MAXIMALLY EXPOSED INDIVIDUAL:

$\begin{array}{rccc} & \text { Local } & \text { Regional } & \text { Imported } \\ \text { Vegetable: } & 1.000 & 0.000 & -0.000 \\ \text { Meat: } & 1.000 & 0.000 & 0.000 \\ \text { Mi } 1 \mathrm{k}: & 1.000 & 0.000 & 0.000\end{array}$

VALUES FOR RADIONUCLIDE-INDEPENDENT VARIABLES:

HUMAN INHALATION RATE

Cubic centimeters/hr $\quad 9.70 \mathrm{E}+05$

SOIL PARAMETERS

Effective surface density ( $\mathrm{kg} / \mathrm{sq} \mathrm{m}$. dry weight)

(Assumes $15 \mathrm{~cm}$ plow layer) $2.24 \mathrm{E}+02$

BUILOUP TIMES

For activity in soil (years)

For radionuclides deposited on ground/water (days) $\quad \begin{aligned} & 5.00 \mathrm{E}+01 \\ & 1.83 \mathrm{E}+04\end{aligned}$

DELAY TIMES .

Ingestion of pasture grass by animals (hr) $\quad 0.00 \mathrm{E}+00$

Ingestion of stored feed by animals (hr) $\quad 2.40 \mathrm{E}+03$

Ingestion of leafy vegetables by man $(h r) \quad 2.40 \mathrm{E}+01$

Ingestion of produce by man (hr) $1.20 \mathrm{E}+02$

Transport time from animal feed-milk-man (day) $\quad 2.00 \mathrm{E}+00$

Time from s.laughter to consumption (day) $1.50 \mathrm{E}+01$

WEATHERING

Removal rate constant for physical loss (per hr) 3.00E-03

CROP EXPOSURE DURATION

Pasture grass (hr)

Crops/leafy vegetables (hr)

$7.20 \mathrm{E}+02$

$2.16 \mathrm{E}+03$

AGRICULTURAL PRODUCTIVITY

Grass-cow-mi lk-man pathway $(\mathrm{kg} / \mathrm{sq} \mathrm{m}) \quad 3.00 \mathrm{E}-01$

Produce/leafy veg for human consumption $(\mathrm{kg} / \mathrm{sq} \mathrm{m}) \quad 2.00 \mathrm{E}+00$ 
Table A-6. Exposure and Consumption Data for the Hanford Site.

(2 of 2 sheets)

FALLOUT INTERCEPTION FRACTIONS

Vegetables

Pasture

2. $50 \mathrm{E}-01$

$2.50 \mathrm{E}-01$

GRAZING PARAMETERS

Fraction of year animals graze on pasture

Fraction of daily feed that is pasture grass when animal grazes on pasture

7.50E-01

$1.00 \mathrm{E}+00$

ANIMAL FEED CONSUMPTION FACTORS

Contaminated feed/forage ( $\mathrm{kg} /$ day, dry weight)

$1.56 \mathrm{E}+01$

DAIRY PRODUCTIVITY

Mi $7 k$ production of cow (L/day)

$1.10 \mathrm{E}+01$

MEAT ANIMAL SLAUGHTER PARAMETERS

Muscle mass of animal at slaughter $(\mathrm{kg})$

Fraction of herd slaughtered (per day)

2. $.00 \mathrm{E}+02$

$3.81 \mathrm{E}-03$

DECONTAMINATION

Fraction of radioactivity retained after washing

for leafy vegetables and produce

1. $00 \mathrm{E}+00$

FRACTIONS GROWN IN GARDEN OF INTEREST

Produce ingested

Leafy vegetables ingested

$1.00 E+00$

1. $00 \mathrm{E}+00$

INGESTION RATIOS:

IMMEDIATE SURROUNDING AREA/TOTAL WITHIN AREA Vegetables

Meat

$1.00 E+00$

Milk

1. $00 E+00$

MINIMUM INGESTION FRACTIONS FROM OUTSIDE AREA

(Minimum fractions of food types from outside

area listed below are actual fixed values.)

Vegetables

Meat

Milk

$0.00 E+00$

$0.00 \mathrm{E}+00$

$0.00 \mathrm{E}+00$

HUMAN FOOD UTILIZATION FACTORS

Produce ingestion $(\mathrm{kg} / \mathrm{y})$

Milk ingestion $(L / y)$

Meat ingestion $(\mathrm{kg} / \mathrm{y})$

Leafy vegetable ingestion $(\mathrm{kg} / \mathrm{y}$ )

2. $20 E+02$

$2.70 \mathrm{E}+02$

$9.80 \mathrm{E}+01$

$3.00 \mathrm{E}+01$

SWIMMING PARAMETERS

Fraction of time spent swimming

Dilution factor for water $(\mathrm{cm})$

$1.00 \mathrm{E}-02$

1. $00 \mathrm{E}+00$ 


\section{DOE/RL-98-33}

Table A-7. Hanford Site Meteorological Data - General Site Information.

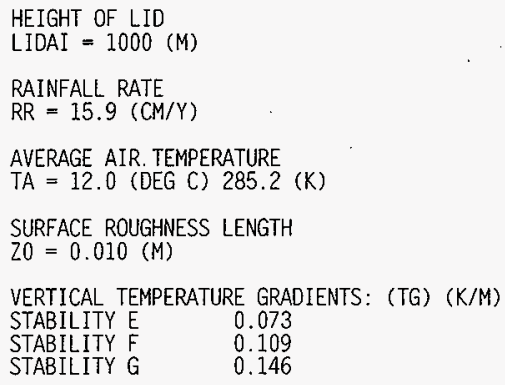




\section{DISTRIBUTION}

\section{Number of Copies}

\section{OFFSITE}

U.S. Environmental Protection Agency, Region 10 1200 Sixth Avenue

Seattle, WA 98101

G. M. Leitch (5)

AT-081

A. Frankel

R. W. Poeten

AT-081

6

Washington State Department of Health

Air Emissions and Defense Waste Section

Division of Radiation Protection

P. O. Box 47827

Olympia, WA $98504-7827$
A. W. Conklin (5)
J. E. Erickson

Washington State Department of Ecology

M. A. Wilson

U.S. Department of Energy-Headquarters

R. F. Pelletier (3)

EH-232

M. K. Harmon

K. C. Duvall

Confederated Tribes of the Umatilla Indian Reservation P. O. Box 638

Pendleton, OR 97801

J. R. Wilkinson 


\section{DISTRIBUTION (continued)}

Number of Copies

\section{OFFSITE}

1

Nez Perce Tribe

Environmental Restoration and Waste Management

P. O. Box 365

Lapwai, ID 83540

D. L. Powaukee

1

Yakama Indian Nation

Environmental Restoration Waste Management Program P.O. Box 151

Toppenish, Washington 98948

R. Jim

\section{ONSITE}

16

U.S. Department of Energy

Richland Operations Office

G. M. Bell

D. W. Bowser

A2-22

R. F. Brich

H0-12

S. E. Clarke

A5-15

J. B. Hall

J. E. Mecca

R3-81

B. M. Pangborn

A5-55

J. E. Rasmussen

A5- 15

H. M. Rodriguez (5)

D. C. Ward

A. H. Wirkkala

DOE-RL Public Reading Room

H2-53 
DISTRIBUTION (continued)

\section{Number of Copies}

\section{ONSITE}

17

Pacific Northwest National Laboratory

E. J. Antonio K3-54

M. Y. Ballinger P7-68

E. G. Damberg P7-68

R. L. Dirkes K6-75

W. T. Farris K3-54

R. W. Hanf, Jr. K6-75

G. R. Hoenes $\quad$ P7-79

S. J. Jette P7-68

G. W. McNair $\quad$ P7-75

T. W. Moon P7-79

K. Rhoads K3-54

$\begin{array}{ll}\text { G. A. Simiele } & \text { P7-79 }\end{array}$

L. H. Staven K3-54

M. J. Sula P7-68

H. T. Tilden II P7-79

R. K. Woodruff $\quad$ P7-68

PNNL Reference Library P8-55

8 Bechtel Hanford, Inc.

R. G. Egge T7-05

M. E. Greenidge $\quad$ X5-54

R. J. Landon H0-18

D. W. Long T7-05

M. R. Morton H0-18

J. E. Rugg S3-21

J. G. Woolard H0-17

J. P. Zoric X5-57

$6 \quad$ Fluor Daniel Hanford, Inc.

President's Office H5-20

W. D. Adair H6-21

J. A. Bates H6-23

S. M. Price H6-23

D. G. Ranade H6-23

B. D. Williamson B3-15 
DOE/RL-98-33

\section{DISTRIBUTION (continued)}

\section{Number of Copies}

ONSITE

1

CH2M Hill, Inc.

E. T. Coenenberg

H9-11

10

B\&W Hanford Company

J. M. Barnett

L1-05

T. G. Beam

S6-51

J. E. Bramson

T5-54

N. R. Dahl

N2-57

T. A. Dillhoff

$\mathrm{N} 2-57$

D. L. Johnson

S6-01

G. J. LeBaron

S6-19

D. E. Rasmussen

$\mathrm{N} 1-47$

R. D. Weissenfels

S6-51

D. L. Wiegand

T5-54

2

DE\&S Hanford. Inc.

R. G. Gant

$\mathrm{X} 3-79$

D. J. Watson

$\mathrm{X} 3-79$

DynCorp Tri-Cities Services, Inc.

B. J. Dixon

B4-20

11

Lockheed Martin Hanford Corporation

M. S. Allen

S7-01

D. J. Carrell

R1-51

G. M. Crummel

R1-51

D. L. Dyekman

T4-08

K. A. Elsethagen

S5-03

B. G. Erlandson

R2-36

P. C. Miller

R1-51

R. P. Raven

R1-51

K. S. Tollefson

R1-51

Distr-4 


\section{DISTRIBUTION (continued)}

Number of Copies

ONSITE

1

Lockheed Martin Services, Inc.

Central Files

B1-07

1

Numatec Hanford Corporation

R. A. Kaldor

H5-25

103

Waste Management Federal Services of Hanford, Inc.

B. M. Barnes

T4-04

R. J. Boom

T6-12

M. W. Bowman

S6-72

H. C. Boynton

T4-52

J. R. Buckley

T3-04

B. L. Curn

H6-36

W. E. Davis

H6-36

L. P. Diediker (75)

H6-36

D. P. Fasset

T3-28

D. L. Flyckt

S6-71

T. P. Frazier

H6-25

D. W. Fritz

H6-36

B. P. Gleckler

H6-36

W. E. Green

H6-36

E. M. Greager

H6-36

R. D. Haggard

H6-25

D. L. Halgren

L6-04

J. S. Hill

H6-25

N. A. Homan

H6-25

R. E. Johnson

H6-25

L. D. Kamberg

H6-25

J. J. Luke

H6-25

J. A. Morrison

S3-31

J. K. Perry

H6-25

D. L. Renberger

T3-03

R. W. Szelmeczka

L6-05

D. B. Van Leuven

H6-10

G. T. Wells

H6-36

J. A. Winterhalder

H6-21

Distr-5 


\section{DISTRIBUTION (continued)}

\section{Number of Copies}

\section{ONSITE}

5

\section{Waste Management Federal}

Services, Inc. Northwest Operations

J. J. Dorian

H1-13

A. R. Johnson

H1-13

B. M. Markes

H1-13

S. J. McKinney

C. J. Perkins
H1-12

H1-12 